\title{
Mixing and Interfacial Composition in Polymer/Fullerene Thin Films
}

\author{
Hynes, Elizabeth L.
}

How to cite:

Hynes, Elizabeth L. (2018) Mixing and Interfacial Composition in Polymer/Fullerene Thin Films. Doctoral thesis, Swansea University.

http://cronfa.swan.ac.uk/Record/cronfa51642

Use policy:

This item is brought to you by Swansea University. Any person downloading material is agreeing to abide by the terms of the repository licence: copies of full text items may be used or reproduced in any format or medium, without prior permission for personal research or study, educational or non-commercial purposes only. The copyright for any work remains with the original author unless otherwise specified. The full-text must not be sold in any format or medium without the formal permission of the copyright holder. Permission for multiple reproductions should be obtained from the original author.

Authors are personally responsible for adhering to copyright and publisher restrictions when uploading content to the repository.

Please link to the metadata record in the Swansea University repository, Cronfa (link given in the citation reference above.)

http://www.swansea.ac.uk/library/researchsupport/ris-support/ 


\title{
Mixing and interfacial composition in polymer/fullerene thin films
}

\author{
Elizabeth Hynes \\ Submitted to Swansea University in fulfillment of the requirements for the \\ degree of Doctor of Philosophy \\ Swansea University \\ 2018
}




\begin{abstract}
Organic photovoltaic (OPV) devices use polymers and small-molecules, such as fullerenes, and offer an alternative to conventional inorganic devices. To achieve commercial success OPV devices require well-controlled morphologies and increased device lifetimes. The film morphology of these devices is complex: both mixing and crystallisation can occur. In this thesis, the fundamental behaviour of two model polymer/fullerene systems is investigated. The impact of parameters such as film thickness, molecular weight (MW) of the polymer and annealing temperature, on crystallisation and mixing are examined.

The effect of MW on the mixing and interface width (between phases with different compositions) in fullerene/polymer systems is presented. These effects are examined in polymer/fullerene bilayers that are annealed at a range of temperatures close to or a few tens of degrees above the (bulk) glass transition temperatures of the materials. Neutron reflectivity is used to probe the composition profile within bilayer thin-films, and optical microscopy and atomic force microscopy (AFM) are used to observe crystal morphology. The fullerenes used are phenyl-C60-butyric acid methyl ester (PCBM) and bis-adduct phenyl-C60-butyric acid methyl ester (bis-PCBM). The polymer used is polystyrene (PS) which is chosen due to its low polydispersity and well-characterised behaviour in thin-films. Stable bilayer composition profiles are observed after annealing, and significant evidence is found for an MW dependence of the interfacial width. The observed behaviour supports the hypothesis that these systems represent a liquid-liquid equilibrium. The behaviour is also found to be broadly in agreement with theoretical predictions.

Preliminary evidence is also presented regarding the impact of fullerene oxidation on mixing in these systems, which is observed to have a significant MW-dependence. Annealing at higher temperatures (well above the glass transition temperature of the materials) results in the growth of large (micron-sized) fullerene crystals in PCBM/PS bilayer samples. The observed morphologies of these crystals are found to be dependent on the PCBM layer thickness.
\end{abstract}




\section{Declaration and Statements}

\section{Declaration}

This work has not previously been accepted in substance for any degree and is not being concurrently submitted in candidature for any degree.

Signed: (candidate)

Date:

\section{Statement 1}

This thesis is the result of my own investigations, except where otherwise stated and that other sources are acknowledged giving explicit references and a bibliography is appended.

Signed: (candidate)

Date:

\section{Statement 2}

I give consent for the thesis, if accepted to be made available online in the University's Open Access Repository and for inter-library loan, and for the title and summary to be made available to outside. organisations

Signed: (candidate)

Date: 


\section{Contents}

Abstract.

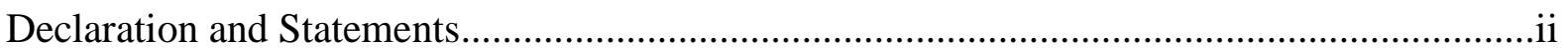

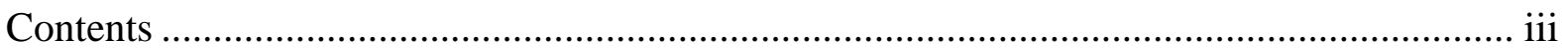

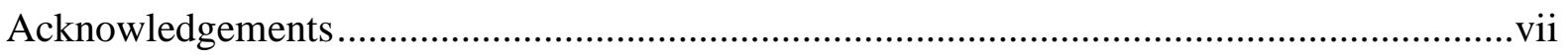

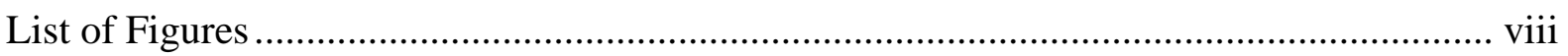

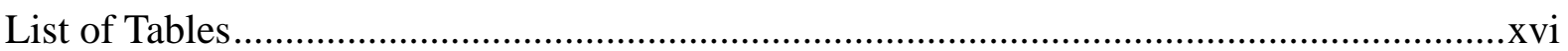

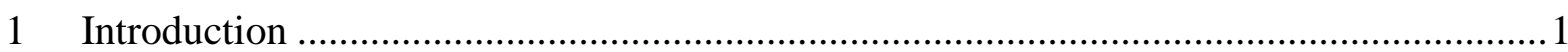

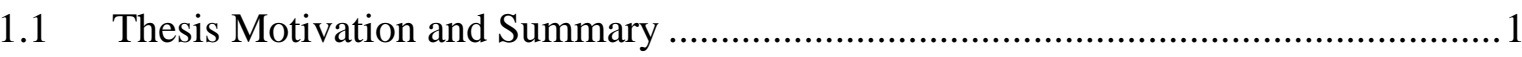

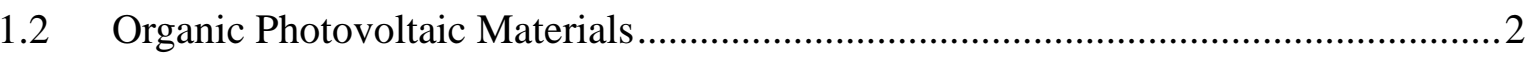

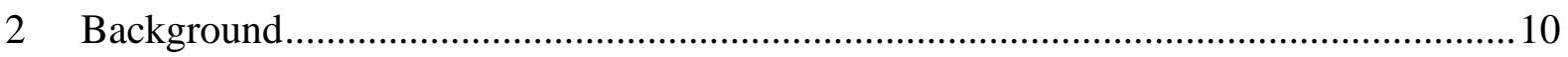

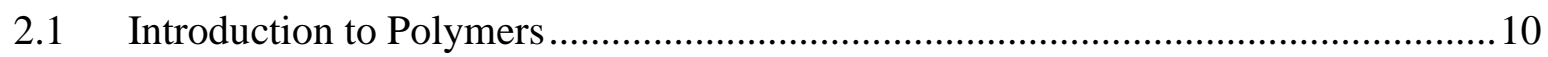

2.1.1 Ideal polymer chains: Polymer random walks ...................................................... 11

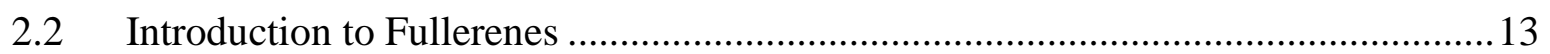

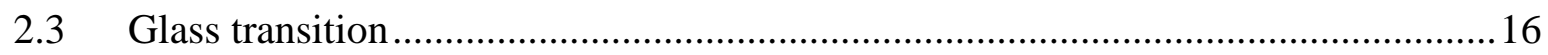

2.4 Thermodynamics of binary liquid un-mixing …................................................ 19

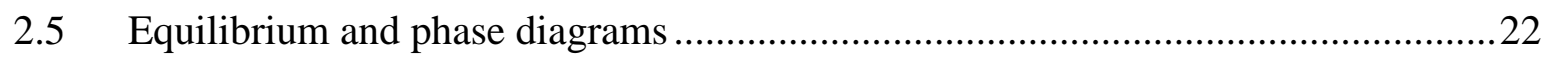

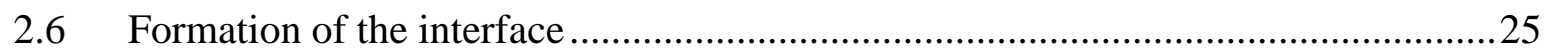

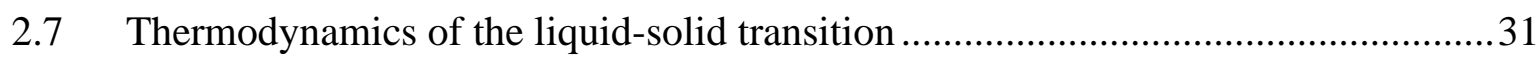

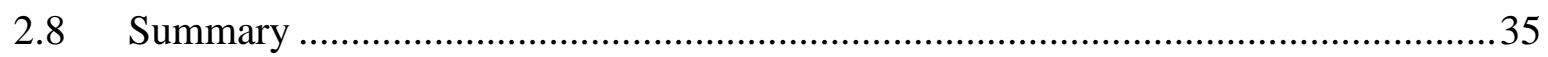

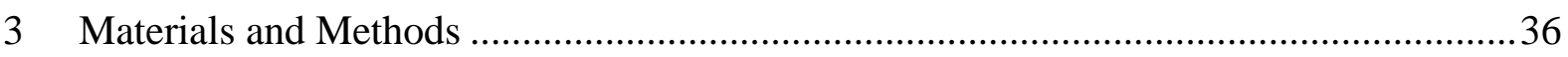

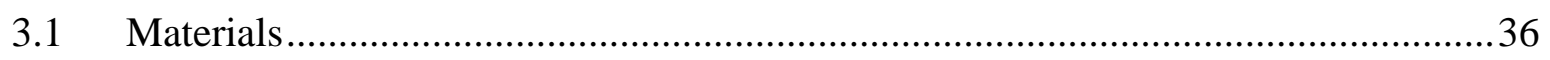

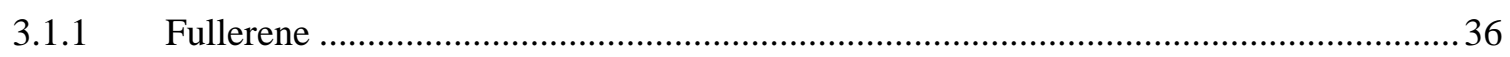

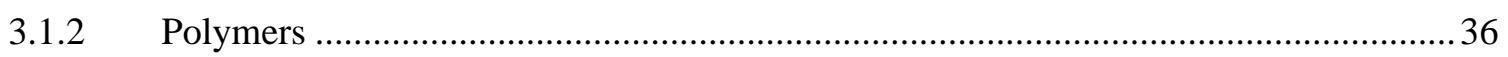

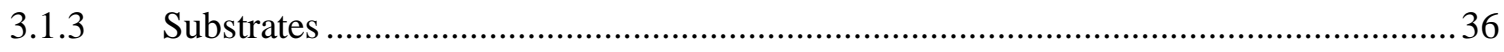

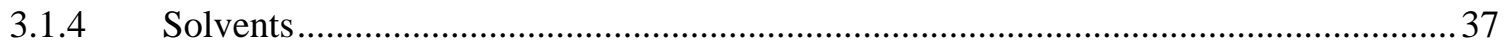

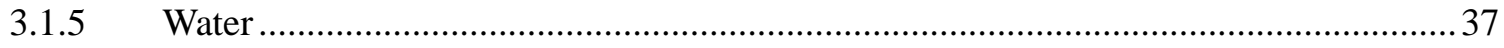

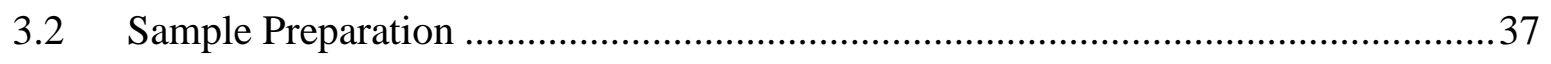

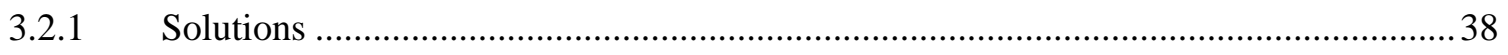

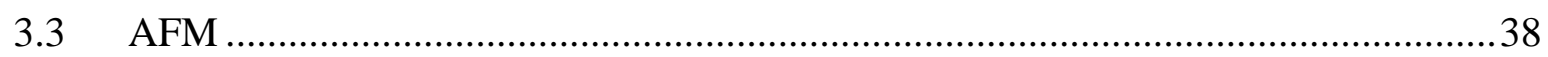

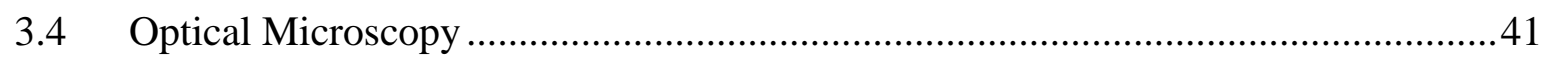

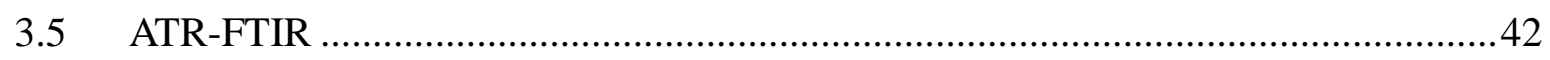

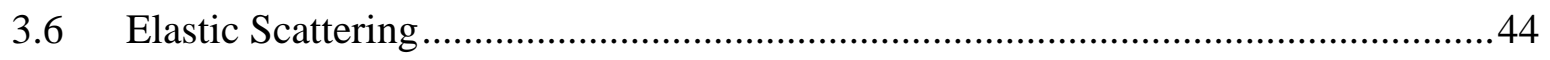

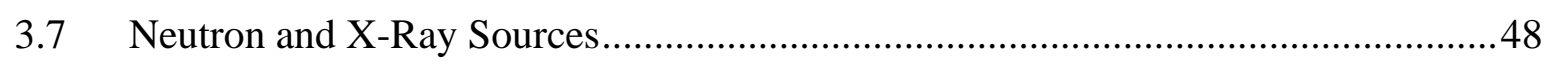

3.8 Neutron Reflectometers and Instrumental Setup ..............................................50 


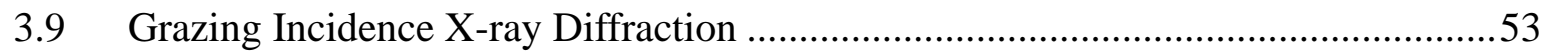

3.10 Neutron Reflectivity: Weak and Strong Scattering ...............................................56

3.11 Neutron Reflectivity: Extracting fit parameters .....................................................58

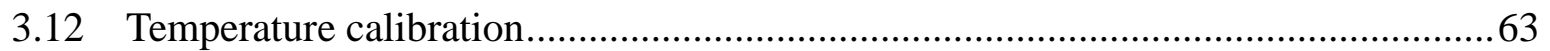

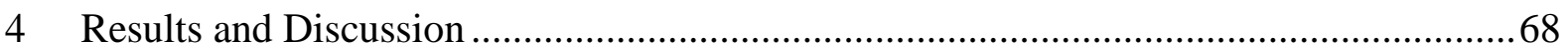

4.1 The impact of molecular weight on the mixing and interfacial width in PCBM/PS

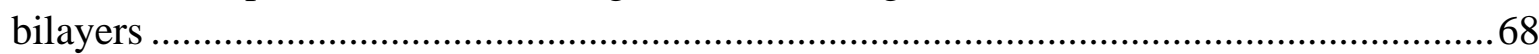

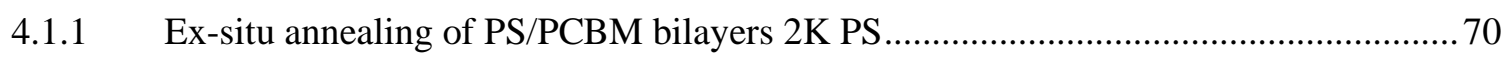

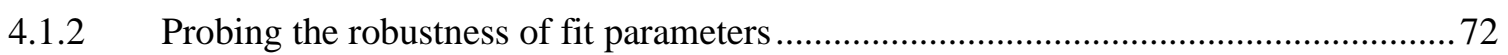

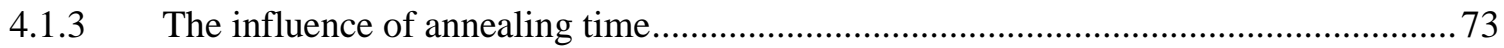

4.1.4 Ex-situ annealing of PS/PCBM bilayers 3.5k and 5k PS ............................................. 74

4.1.5 Ex-situ annealing of PS/PCBM bilayers PS 20k, 100k, 200k and 300k ..................... 75

4.1.6 The impact of sample-to-sample variation and choice of reflectometer on reflectivity

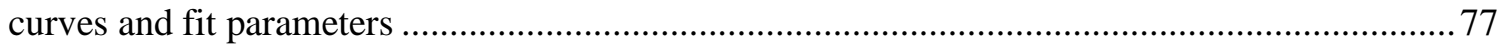

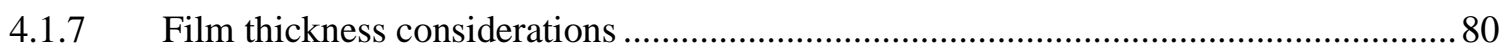

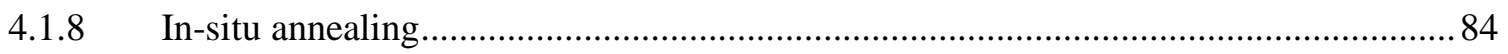

4.1.9 The influence of PS MW on interfacial roughness and coexisting compositions....... 100

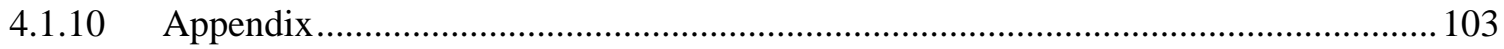

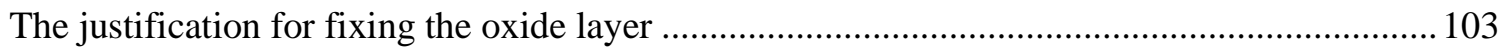

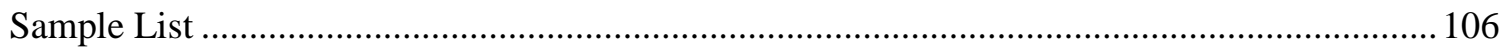

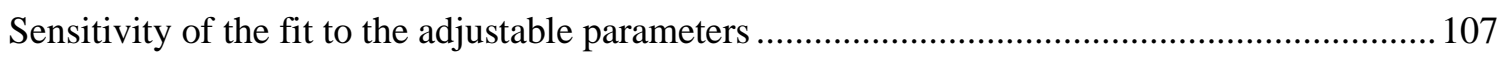

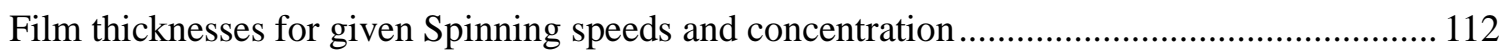

The impact of lateral inhomogeneities in the samples on NR curves ........................................ 113

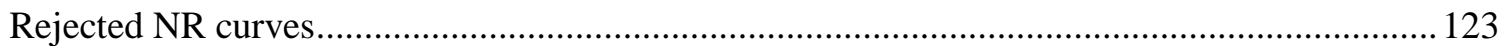

Testing the robustness of the fitted parameters: top layer SLD and intefacial roughness........... 126

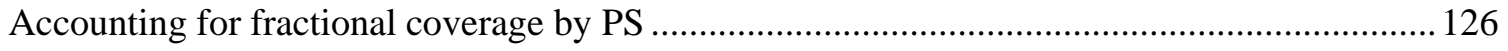

4.2 The impact of molecular weight on mixing and interfacial width in bis-PCBM/PS

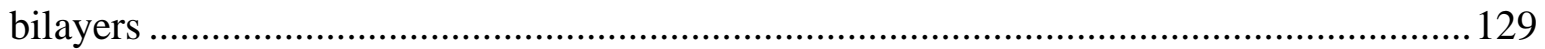

4.2.1 Bilayer fits for 2k, 3.5k, 5k, 20k and 100k PS ex-situ samples ............................... 129

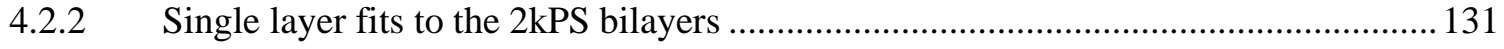

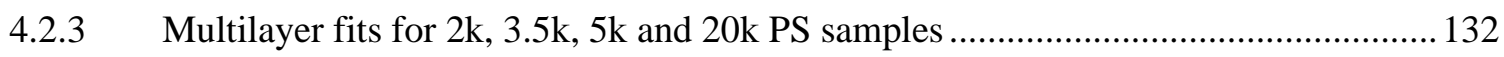

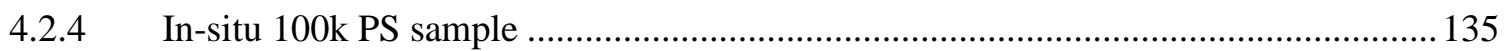

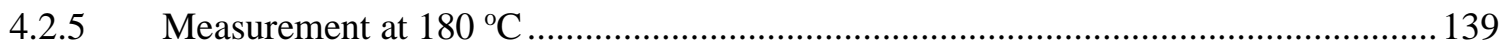

4.2.6 The influence of PS MW on layer composition and interfacial roughness in

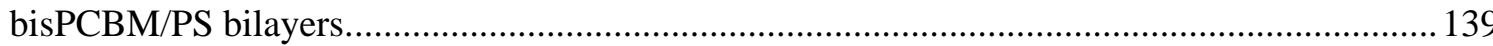

4.2.7 Overall conclusions for bis-PCBM/PS and PCBM/PS …........................................ 144

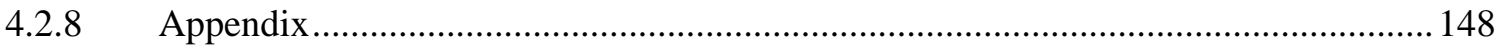

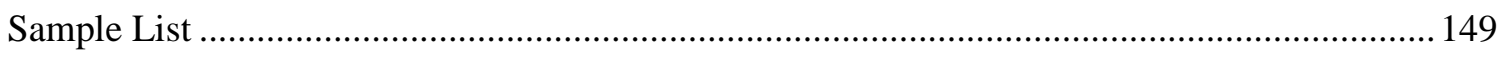


Assessing the level of lateral inhomogeneities on bis-PCBM/PS bilayers 149

Film thickness

4.3 The effect of different storage environments on annealed PCBM/PS composition

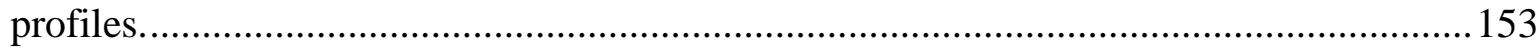

4.3.1 Aged/oxidised PS/PCBM bilayer samples............................................................... 155

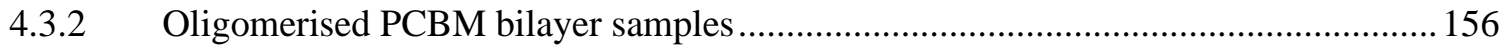

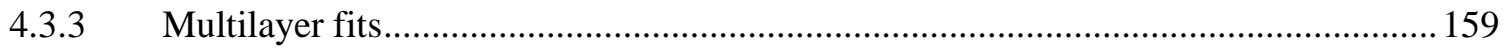

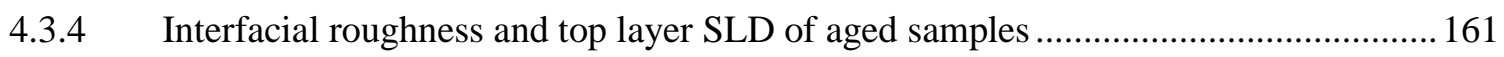

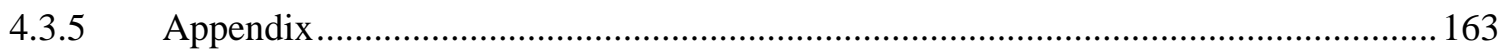

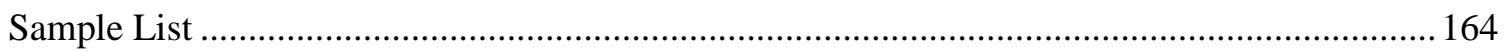

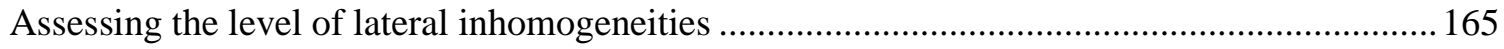

4.4 Bilayers using a non-fullerene acceptor and a conjugated polymer....................... 166

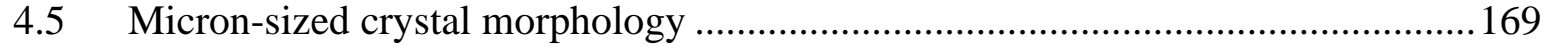

4.5.1 'Phase diagrams' for different layer thicknesses, MW, and temperature. .................... 170

4.5.2 Temperature dependence of fan morphology .............................................................. 176

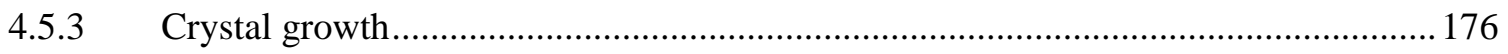

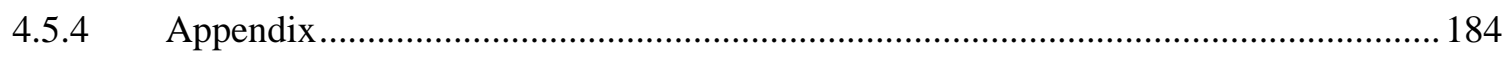

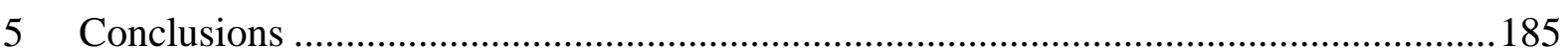

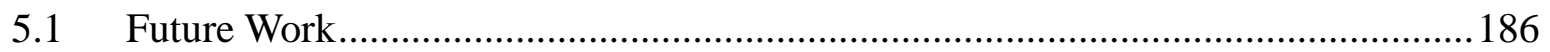

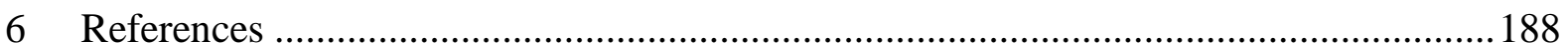





\section{Acknowledgements}

I would like to thank the following people and organisations for support during my $\mathrm{PhD}$. I would like to thank Phillipe Gutfreund ${ }^{1}$ at the ILL and Becky Welbourne ${ }^{2}$ at ISIS for facilitating our experiments. I would like to thank Andrew Parnell ${ }^{3}$ at Sheffield University for use of the Sheffield GIXD apparatus. I would like to thank Joao Cabral ${ }^{4}$ and Seb Pont ${ }^{4}$ for performing oligomerisation of some samples at Imperial College London. I would like to thank Zhe $\mathrm{Li}^{5}$ and Harrison ${ }^{5}$ Lee at SPECIFIC, Swansea University for oxidising some samples and providing some materials. I would like to thank Alan Dunbar ${ }^{3}$ at Sheffield University for collaboration at Diamond. I would like to thank Huw Summers ${ }^{5}$ and Paolo Bertoncello $^{5}$ for use of their optical microscopes. I would like to thank Richard Cobley ${ }^{5}$, Alex Lord ${ }^{5}$ and Chris Wright ${ }^{5}$ for use of their AFMs. I would like to thank Anthony Higgins, Joao Cabral and Fabrizia Foglia ${ }^{4}$ for help carrying out the neutron reflectivity measurements.

I would like to thanks Anthony Higgins ${ }^{5}$ who performed some of the FTIR experiments and some of the temperature calibrations, as well as for being my supervisor and for giving me plenty of feedback and very good advice. I would also like to thank all of my friends and family for their support and encouragement. I would also like to thank my family, especially my sister, mother, father and brother, for helping me with proofreading.

${ }^{1}$ Institut Laue-Langevin, 71 Avenue des Martyrs, 38000 Grenoble, France

${ }^{2}$ ISIS, Rutherford Appleton Laboratory, Harwell Oxford, Didcot OX11 0QX

${ }^{3}$ Department of Physics and Astronomy, The University of Sheffield, Western Bank, Sheffield S10 2TN

${ }^{4}$ Department of Chemical Engineering and Centre for Plastic Electronics, Imperial College London, London SW7 2AZ

${ }^{5}$ College of Engineering, Swansea University, Bay Campus, Swansea, SA1 8EN 


\section{List of Figures}

Figure 1.2-1: Schematic of the conjugated polymer, polyacetylene, showing the alternating single and double bonds.

Figure 1.2-2: Schematic a heterojunction between electron donating (brown) and electron accepting (blue) materials, within OPV devices. HOMO is the highest occupied molecular orbital, and LUMO is lowest unoccupied molecular orbital. Charge generation occurs when an electron moves fro the HOMO to the LUMO to the acceptor to the electrode and is separated from the hole that moves from the HOMO to the other electrode......

Figure 1.2-3: A flexible OPV module reproduced under the Creative Commons licence from reference

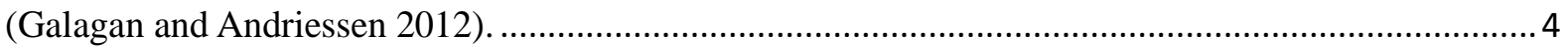

Figure 1.2-4: Left shows a bilayer architecture while right shows a BHJ. ........................................ 6 Figure 1.2-5: Needle-like micron-sized crystals on a glass substrate for 1:1 blend of polymer and PCBM annealed at $190{ }^{\circ} \mathrm{C}$ reproduced with permission from reference(Lindqvist et al. 2013)............8 Figure 1.2-6: Branched crystals formed when PCBM:PCDTBT 2:1 blend annealed at $140{ }^{\circ} \mathrm{C}$ for $1 \mathrm{hr}$ in the dark. Scale bar is $100 \mu \mathrm{m}$, and inset optical image is 20x20um. Reproduced with permission from reference (Z. Li et al. 2013)

Figure 1.2-7: GIXD patterns and optical micrographs (insets) of two PCBM/PS bilayers and a PCBM single layer. The PCBM/PS bilayers were annealed at $170{ }^{\circ} \mathrm{C}$ for 20 minutes ( $\mathrm{h}$ and i) and the PCBM single layer (k) was annealed for 60 minutes. The PS thicknesses were as follows; h) $8 \mathrm{~nm}$, i) $25 \mathrm{~nm}$ PS. All samples had a PCBM layer thickness 20nm (before annealing). The PS MW was 350kDa in all samples. Reproduced with permission from reference (Môn et al. 2015). The scale bar in k) is $20 \mu \mathrm{m}$, and all micrographs are the same magnification. The GIXD patterns are detector maps with the scales indicating qz and qx-y(in units of $\AA^{-1}$ ) as defined in Môn et al (Reproduced with permission

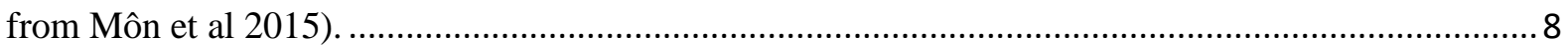

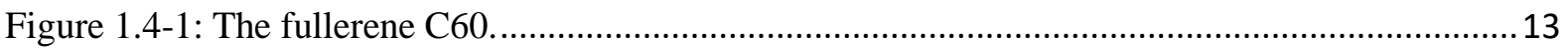

Figure 1.4-2: Chemical structure of PCBM and bis-PCBM reproduced with permission from reference(Brabec et al. 2010)

Figure 1.4-3:The effect of heat treatment on the extent of oligomerisation in PCBM films. The PCBM films were first oligomerised to a maximal $37 \%$, by illumination with visible light in an inert atmosphere. The films were then subjected to an hour of annealing at the temperatures shown. Adapted with permission from reference (Wong, Mcculloch, et al. 2014). The y-axis shows the number fraction of oligomers with-respect-to the total amount of PCBM. The fractions were taken from gel permeation chromatographic analyses comparing the size of the signal from oligomerised PCBM to pristine PCBM.

Figure 1.5-1: The discontinuity in heat capacity indicates the glass transition temperature. Reproduced

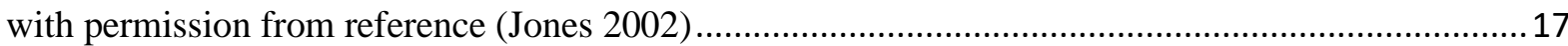
Figure 1.5-2: The glass transition temperature as a function of molecular weight for polystyrene, from Santangelo and Roland (Santangelo and Roland 1998). $\mathrm{M}_{\mathrm{e}}$ is the entanglement molecular weight. ... 18 Figure 1.5-3: Glass transition temperature as a function of film thickness (h) for polystyrene. Reproduced with permission from reference (J. A. Forrest and Dalnoki-Veress 2001)...

Figure 1.6-1: Lattice describing conformation of a polymer in a solution or mixture with a small molecule. Reproduced with permission from reference (Ender et al. 2012).

Figure 1.7-1: Free energy curves for (a) unstable and (b) stable configurations. Reproduced with permission from reference (R. Colby, M. Rubinstein, 2003). 
Figure 1.7-2: A free energy curve with two minima reproduced with permission from reference (R. A.

L. Jones 2002).

Figure 1.7-3: Example free energy curves (Gibbs free energy of mixing) for different temperatures for $\mathrm{NA} \neq \mathrm{NB}$ and $\chi=(5 \mathrm{~K}) / \mathrm{T}$. Reproduced with permission from reference (Y. Zhao et al. 2011)..........24

Figure 1.7-4: A phase diagram for a symmetric mixture of small molecules showing the stable, metastable and unstable regions. Reproduced with permission from reference(R. A. L. Jones 2002). 25

Figure 1.8-1: Schematic of protruding loops illustrating molecular mixing at the interface. .26

Figure 1.8-2: Plot of equation (2.49) from Broseta et al (Broseta et al. 1990) for various degrees of polymerisation, $\mathrm{N}$, on either side of a polymer-polymer interface with $\chi=2$. The MW of 1 monomer is 633Da.

Figure 1.8-3: Polymer-polymer interfacial width from equation (2.48) from Tang and Freed, (R. A. L. Jones and R. W. Richards, W. 1999; Tang and Freed 1991) for a symmetric polymer interface(i.e. $\mathrm{NA}=\mathrm{NB}=\mathrm{N}$ ). The $\mathrm{x}$-axis has been converted from number of monomers, $\mathrm{N}$, into MW for the full range of PS MWs used in this thesis. The curve is also shown for $\mathrm{N}=\mathrm{Nave}$, with $\mathrm{N}_{\mathrm{A}}$ equal to the number of monomers in a PS chain and $\mathrm{NB}=1 . \mathrm{N}_{\mathrm{A}}, \mathrm{N}_{\mathrm{B}}$ and $\chi$ are referenced to the volume of a PCBM monomer ( $\left.\mathrm{V}_{\mathrm{PCBM}}\right)$. The 'effective' statistical segment length, $b_{\text {eff, }}$, is given by $\left(l_{\mathrm{k}} l_{\mathrm{ref}}\right)^{0.5}$, where $\mathrm{l}_{\mathrm{k}}$ is the Kuhn length of PS and $1_{\text {ref }}$ is a reference length equal to $\left(\mathrm{v}_{\mathrm{PCBM}} \mathrm{l}_{\mathrm{k}}\right) / \mathrm{v}_{\mathrm{k}}$ (with $\mathrm{v}_{\mathrm{k}}$ equal to the volume of a PS Kuhn monomer). This plot for $\chi=2$, corresponds to the experimental situation in section 4.1. The 'effective' statistical segment length is the length required to give the correct prefactor to equation (2.48), given that the $\chi$ parameter in the denominator is referenced to the size of a PCBM molecule (rather than the size of a PS monomer).

Figure 1.9-1: $\Delta \mathrm{Gv}$ is the energy change in the bulk and $\Delta \mathrm{Gs}$ is the energy change of the surface.$\Delta \mathrm{G}$ is the total gibbs free energy change. Reproduced with permission from reference (Perrin, Musa, and Steed 2013).

Figure 1.9-2: This shows various crystal sizes and branching for different blend rations (indicated on the vertical axis) for different annealing temperatures (indicated on the horizontal axis). Reproduced with permission from reference (Swinnen et al. 2006).

Figure 1.9-3: The progression from a single needle (A) to a branched needle (B) to a spherulite(E).

Reproduced with permission from reference (Granasy et al. 2005).................................................. 34

Figure 2.2 2: Chemical structure of PCBM and bis-PCBM reproduced with permission from reference (Brabec et al. 2010)

Figure 2.3-1: The tip responds to the force felt by the atoms on the surface and generates the trace, B.

Reproduced with permission from reference (Binnig and Quate 1986). . .39

Figure 2.3-2: Schematic diagram of an AFM sowing the laser light hitting the tip before being reflected into the position sensitive detector (PSD).

Figure 2.3-3: Diagram of forces felt by the tip as it approaches the sample and which region applies to each scanning mode.

Figure 2.5-1: FTIR-ATR spectra of a) PCBM and b) PS films before and after annealing for 30 minutes at the temperatures shown, in a vacuum oven. The PCBM absorbance spectrum after annealing has been scaled by a constant factor so that the peak at $1737 \mathrm{~cm}^{-1}$ has the same height in the spectra before and after annealing. The force applied to the samples in all measurements was kept at a similar value. The differences in background level in the spectra before and after annealing are likely to be due to differences in the applied force pressing the sample onto the ATR crystal, and the resulting contact area between sample and AFM crystal. Especially in the case of PCBM in which the amount of material scraped onto the crystal was not rigorously controlled.

Figure 2.6-1: Geometry of momentum transfer $\mathrm{Q}$ in terms of wave vectors. Double-angle is used for convention. The plot shows the $\mathrm{x}-\mathrm{z}$ plane, where the incident beam has its wavevector pointing along the $\mathrm{z}$ direction.

Figure 2.6 3: Polymer-polymer interfacial width from equation (2.48) from Tang and Freed (R. A. L. Jones and R. W. Richards, W. 1999; Tang and Freed 1991) for a symmetric polymer interface(i.e. 
N_A=N_B=N). The $x$-axis has been converted from number of monomers, N, into MW for the full range of PS MWs used in this thesis. The curve is also shown for $\mathrm{N}=\mathrm{N}$ aave, with NA equal to the number of monomers in a PS chain and $\llbracket \mathrm{N} \rrbracket \_B=1$. NA, NB and $\square \square$ are referenced to the volume of a PCBM monomer (vPCBM). The 'effective' statistical segment length, beff, is given by (lklref)0.5, where $1 \mathrm{k}$ is the Kuhn length of PS and lref is a reference length equal to (vPCBMlk)/vk (with vk equal to the volume of a PS Kuhn monomer).This plot for $\square=2$, corresponds to the experimental situation in section 4.1. The 'effective' statistical segment length is the length required to give the correct prefactor to equation (2.48), given that the $\square$ parameter in the denominator is referenced to the size of a PCBM molecule (rather than the size of a PS monomer).

Figure 3.8-1: Detector setup at Figaro in the ILL for TOF mode.

Figure 3.11-1: Scattering length values as a function of atomic number. Reproduced with permission

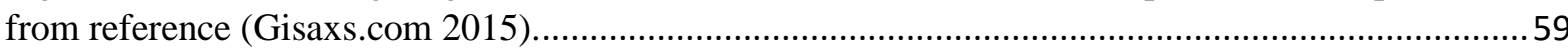

Figure 3.11-2: Diagram of geometric relation between k, $\theta$ and $\mathrm{q}$. ................................................... 60

Figure 3.11-3: Reflectivity curves for a bare silicon substrate (a) and a silicon substrate with a

50nmlayer on top (b)adapted with permission from reference (X. Zhang et al. 2016).

Figure 3.11-4: A model detector map (left) showing the location of a Yoneda peak in relation to the specular scattering seen for $\theta=2.5$, reproduced with permission from reference (D. W. James 2011). Specular and off-specular neutron reflectivity (right) measured from an annealed polymer/polymer bilayer on a silicon substrate. Sample characteristics on this sample were: bottom layer had an SLD of $6.83 \times 106$ and a thickness of $480 \AA$, the top layer had an SLD of 5.3×10-7 and a thickness of 1000A. These were measured by a combination of AFM measurements and fits to the specular and offspecular scattering. The lateral roughness at the silicon/bottom layer was $3 \AA$ or $4 \AA$ ( $3 \AA$ is the fit to the NR data, and $4 \AA$ is the rms measurement from AFM). The total roughness at the buried polymer/polymer interface in this sample was $22 \AA$ (consisting of $15 \AA$ lateral roughness and $16 \AA$ intrinsic roughness). Reproduced with permission from reference (D. James et al. 2015). .................63 Figure 3.12-1:Setpoint of heater and temperature on sample surface, for a 2 inch diameter silicon wafer using the in-situ annealing procedure. Overshoots in the sample surface temperature are seen for jumps of 20 degrees (from $80^{\circ} \mathrm{C}$ to $180^{\circ} \mathrm{C}$ ) and 10 degrees (from $120^{\circ} \mathrm{C}$ to $150^{\circ} \mathrm{C}$ ). The error on the sample surface is $+/-1.5^{\circ} \mathrm{C}$

Figure 3.12-2: This graph shows the cooling of the sample surface from $180^{\circ} \mathrm{C}$ and $140^{\circ} \mathrm{C}$ to $60^{\circ} \mathrm{C}$ when the setpoint is dropped to $60^{\circ} \mathrm{C}$. Error on sample surface of $+/-1.5^{\circ} \mathrm{C}$

Figure 3.12-3: Schematic diagram of how the samples were attached to the stage during the in-situ measurements.

Figure 3.12-4: Demonstration of the dependence of the temperature on the sample surface on how tightly the sample is screwed onto the surface.

Figure 3.12-5: Calibration of vacuum oven for annealing temperatures used in ex-situ samples (performed by Anthony Higgins, Swansea University).The different colours represent the different set-points given in Table 2 (in ascending order from red to orange). Different symbols represent repeat measurements (including at slightly different locations within the oven)

Figure 4.1-1:NR curves (left) and SLD profiles (right) for PCBM single layer and unannealed 2k PCBM/PS bilayer.

Figure 4.1-2: NR curves for PCBM/PS bilayers with PS MW=2k Da annealed at various temperatures. Curves are offset vertically for clarity in this and many subsequent reflectivity figures.

Figure 4.1-3:SLD profiles for PCBM/PS bilayers with PS MW=2k Da annealed at various temperatures.

Figure 4.1-4: Two PCBM/ PS MW=2k Da samples with the oxide layer parameters allowed to vary, in comparison to fixed oxide layer fits. NR curves (top) and SLD profiles (bottom). The sample annealed shown that was annealed at $125^{\circ} \mathrm{C}$ is one of the double thickness samples discussed in section 4.1.7. 
Figure 4.1-5: Interfacial roughness (left) and SLD of top layer (right) for bilayer samples of $\mathrm{PCBM} / \mathrm{PS}$ with $\mathrm{MW}=2 \mathrm{k}$ ex $=$ situ annealed at various times at $120^{\circ} \mathrm{C}$.

Figure 4.1-6: NR curves for PCBM/PS bilayers with PS MW=3.5k Da (top) and 5k Da (bottom) annealed at various temperatures.

Figure 4.1-7: SLD profiles for PCBM/PS bilayers with PS MW=3.5k Da (left)and 5k Da

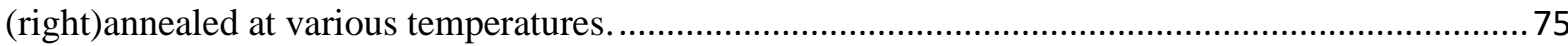

Figure 4.1-8: NR curves for PCBM/PS bilayers with PS MW=20k Da annealed at various temperatures

Figure 4.1-9: SLD profile for PCBM/PS bilayers with PS MW=20k Da annealed at various

temperatures.

Figure 4.1-10: NR curves for PCBM/PS bilayers with PS MW=100k, 200k (top) and 300k (bottom)

$\mathrm{Da}$ annealed at various temperatures.

Figure 4.1-11: SLD profiles for PCBM/PS bilayers with PS MW=100k, 200k (left) and 300k Da

(right) annealed at various temperatures.

Figure 4.1-12:Data and fits for two different $M W=5 \mathrm{k}$ Da bilayers annealed at the same temperature (a), two different $M W=20 \mathrm{k}$ Da bilayers annealed at the same temperature (b) and the same sample measured twice (c) measured at different reflectometers; Figaro at the ILL, D17 at the ILL or INTER at ISIS.

Figure 4.1-13: SLD profiles for data measured from the reproduced and repeated samples measured at

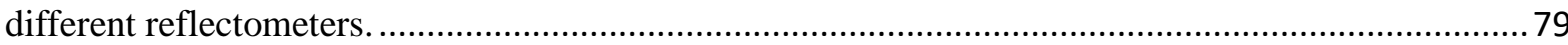

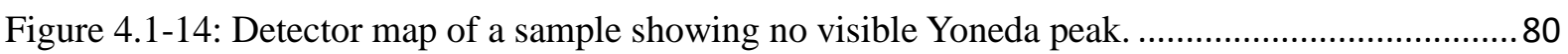

Figure 4.1-15: NR curves and fits for PCBM/PS bilayers with PS MW=2k Da and the thickness of

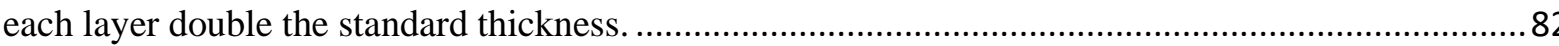

Figure 4.1-16: SLD profiles for PCBM/PS bilayers with PS MW=2k Da and the thickness of each layer thicker than the standard thickness.

Figure 4.1-17:Interfacial roughness post-annealing for a range of post-annealing thicknesses of the bottom layer.

Figure 4.1-18: SLD of the top layer post-annealing for a range of post-annealing thicknesses of the bottom layer.

Figure 4.1-19: In-situ data for samples indicated in the headings. Full NR curves taken before and after annealing, shorter NR curves are 30s kinetic measurement showing distinctive visual changes in the shape of the curve at low Q indicating a change in parameters.

Figure 4.1-20: In-situ SLD profiles for the before and after annealing measurements for the samples indicated in the headings.

Figure 4.1-21: Kinetic measurements of the reflectivity curve of a PCBM single layer, and a PS $\mathrm{MW}=20 \mathrm{k}$ Da single layer.

Figure 4.1-22:The change in fit parameters over time for PCBM/PS MW=2kDa. Each data point is for reflectivity summed over four 30s measurements barring the inset graphs which show data points for individual 30s measurements. Graph a is thickness of the top layer (PS-rich), graph b is the thickness of the bottom layer (PCBM), graph $\mathrm{c}$ is the SLD of the top layer, $d$ is the SLD of the bottom layer, e is the top layer roughness and $f$ is the interfacial roughness.

Figure 4.1-23: The change in fit parameters over time for PCBM/PS MW=3.5kDa. Each data point is for reflectivity summed over four 30s measurements barring the inset graphs which show data points for individual 30s measurements. Graph a is thickness of the top layer (PS-rich), graph $b$ is the thickness of the bottom layer (PCBM), graph $\mathrm{c}$ is the SLD of the top layer, $\mathrm{d}$ is the SLD of the bottom layer, $\mathrm{e}$ is the top layer roughness and $\mathrm{f}$ is the interfacial roughness. .................................................. 90 Figure 4.1-24: The change in fit parameters over time for PCBM/PS MW=5kDa. Each data point is for reflectivity summed over four 30s measurements barring the inset graphs which show data points for individual 30s measurements. Graph a is thickness of the top layer (PS-rich), graph b is the 
thickness of the bottom layer (PCBM), graph $\mathrm{c}$ is the SLD of the top layer, $d$ is the SLD of the bottom layer, $\mathrm{e}$ is the top layer roughness and $\mathrm{f}$ is the interfacial roughness.

Figure 4.1-25 The change in fit parameters over time for PCBM/PS MW=20kDa. Each data point is for reflectivity summed over four 30s measurements barring the inset graphs which show data points for individual 30s measurements. Graph a is thickness of the top layer (PS-rich), graph b is the thickness of the bottom layer (PCBM), graph $\mathrm{c}$ is the SLD of the top layer, $\mathrm{d}$ is the SLD of the bottom layer, $\mathrm{e}$ is the top layer roughness and $\mathrm{f}$ is the interfacial roughness. ............................................92 Figure 4.1-26: The change in fit parameters over time for PCBM/PS MW=100kDa with the top layer having an initial concentration of 5\% PCBM v/v.. Each data point is for reflectivity summed over four 30s measurements barring the inset graphs which show data points for individual 30s measurements. Graph a is thickness of the top layer (PS-rich), graph b is the thickness of the bottom layer (PCBM), graph $\mathrm{c}$ is the SLD of the top layer, $d$ is the SLD of the bottom layer, e is the top layer roughness and $f$ is the interfacial roughness.

Figure 4.1-27:The change in fit parameters over time for PCBM/PS MW=100kDa with the top layer having an initial concentration of $20 \%$ PCBM v/v. Each data point is for reflectivity summed over four 30s measurements barring the inset graphs which show data points for individual 30s measurements. Graph a is thickness of the top layer (PS-rich), graph b is the thickness of the bottom layer (PCBM), graph $\mathrm{c}$ is the SLD of the top layer, $\mathrm{d}$ is the SLD of the bottom layer, e is the top layer roughness and $\mathrm{f}$ is the interfacial roughness.

Figure 4.1-28:The change in fit parameters over time for a PCBM single layer on the right and a PS $\mathrm{MW}=20 \mathrm{kDa}$ single layer on the left.. Each data point is for reflectivity summed over four or five 30s measurements. Graph a is thickness of the PS single layer, graph b is the thickness of the PCBM single layer, graph $\mathrm{c}$ is the SLD of the PS single layer, $d$ is the SLD of the PCBM single layer, e is the PS single layer roughness and $\mathrm{f}$ is the PCBM single layer surface roughness.

Figure 4.1-29: The temperature on the sample surface overlaid against the step in thickness of the bottom layer for PCBM/PS MW=3.5kDa.

Figure 4.1-30: Gaussian interface roughness versus PS molecular weight for PCBM/PS bilayers annealed at various temperatures and times for ex-situ samples.

Figure 4.1-31: The SLD of the top PS rich layer is shown for all of the molecular weight series of PCBM/PS bilayers from ex-situ samples of varying MW. Data points from reference Mon et. al(Môn et al. 2015)

Figure 4.1-32: Free energy curve for an F-H chi value of 2 for a polymer mixture with monomer size being that of a PCBM molecule. The dashed lines show the commom tangent construction for $2 \mathrm{k}$ and 20k-300k PS and the stars indicate experimentally measured compositions.The PS MWs in the legend are weight averages. MW of $1.86 \mathrm{k}$ corresponds to $\mathrm{N}=2.9 ; 2.93 \mathrm{k}$ to $\mathrm{N}=4.6 ; 4.73 \mathrm{k}$ to $\mathrm{N}=7.5 ; 18.5 \mathrm{k}$ to $\mathrm{N}=29.2 ; 111.4 \mathrm{k}$ to $\mathrm{N}=176$; and $278.2 \mathrm{k}$ to $\mathrm{N}=440$. 102

Figure 4.1-33: Parameter values for fixed and varied oxide layers. The same colour represents the same sample.

Figure 4.1-34: Parameter values for fixed and varied oxide layers. The same colour represents the same sample.

Figure 4.1-35: Box and whisker diagram of the average and spread in the magnitude of the change in parameter values between a fixed and a variable oxide layer for all samples. The whisker ends indicate max and min values, the box edges indicate first (lower) and third (upper) quartiles. The middle line of the box shows the median value, and the squares show the mean value. 105

Figure 4.1-36: The change in the fit for altering the value of the bottom layer thickness and keeping the other parameters fixed, which increases the reduced $\chi 2$ value by varying degrees. Data shown is for a PCBM/PS MW=5k bilayer annealed at $135 \mathrm{C}$ for $5 \mathrm{~m}$. 108 Figure 4.1-37: The change in the fit for altering the value of the top layer thickness and keeping the other parameters fixed, which increases the reduced $\chi 2$ value by varying degrees. Data shown is for a $\mathrm{PCBM} / \mathrm{PS} \mathrm{MW}=5 \mathrm{k}$ bilayer annealed at $135 \mathrm{C}$ for $5 \mathrm{~m}$ 
Figure 4.1-38: The change in the fit for altering the value of the interfacial roughness and keeping the other parameters fixed, which increases the reduced $\chi 2$ value by varying degrees. Data shown is for a $\mathrm{PCBM} / \mathrm{PS} \mathrm{MW}=5 \mathrm{k}$ bilayer annealed at $135 \mathrm{C}$ for $5 \mathrm{~m}$.

Figure 4.1-39: The change in the fit for altering the value of the top layer roughness and keeping the other parameters fixed, which increases the reduced $\chi 2$ value by varying degrees. Data shown is for a $\mathrm{PCBM} / \mathrm{PS} \mathrm{MW}=5 \mathrm{k}$ bilayer annealed at $135 \mathrm{C}$ for $5 \mathrm{~m}$ 109

Figure 4.1-40: The change in the fit for altering the value of the bottom layer SLD and keeping the other parameters fixed, which increases the reduced $\chi 2$ value by varying degrees. Data shown is for a $\mathrm{PCBM} / \mathrm{PS} \mathrm{MW}=5 \mathrm{k}$ bilayer annealed at $135 \mathrm{C}$ for $5 \mathrm{~m}$.

Figure 4.1-41: The change in the fit for altering the value of the top layer SLD and keeping the other parameters fixed, which increases the reduced $\chi 2$ value by varying degrees. Data shown is for a $\mathrm{PCBM} / \mathrm{PS} \mathrm{MW}=5 \mathrm{k}$ bilayer annealed at $135 \mathrm{C}$ for $5 \mathrm{~m}$

Figure 4.1-42: Chi-squared contour map for top layer versus interfacial roughnesses for PCBM/PS

$\mathrm{Mw}=2 \mathrm{k}$ Da sample annealed at $120 \mathrm{C}$ for $5 \mathrm{~m}$. Black shows the minimum in chi-squared................111

Figure 4.1-43: Chi-squared contour map for top layer versus interfacial roughness for PCBM/PS

$\mathrm{Mw}=20 \mathrm{k}$ Da sample annealed at $120 \mathrm{C}$ for $5 \mathrm{~m}$. Black shows the minimum in chi-squared..............112 Figure 4.1-44: Micrograph and NR curve for annealed bilayer showing the lateral inhomogeneities on the sample.

Figure 4.1-45:Optical micrograph of samples surface and NR curve and fit for the 2k 145C 2 minutes

sample.

Figure 4.1-46: Optical micrograph of samples surface and NR curve and fit for the 2k 135C 5 minutes sample.

Figure 4.1-47: Micrograph and NR curve for annealed bilayer showing the lateral inhomogeneities on the sample.

Figure 4.1-48: Defect height profiles for lines from Figure 4.1-49.............................................116

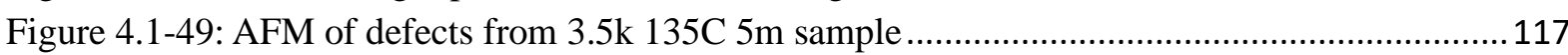

Figure 4.1-50: Micrograph and NR curve for annealed bilayer showing the lateral inhomogeneities on the sample.

Figure 4.1-51: Micrograph and NR curve for annealed bilayer showing the lateral inhomogeneities on the sample.

Figure 4.1-52: Micrograph and NR curve for annealed bilayer showing the lateral inhomogeneities on the sample.

Figure 4.1-53: Top: micrograph of PCBM/PS Mw=2k Da sample annealed at 140C 1m, bottom left, AFM image; bottom right: GIXD image. The rms roughness from AFM for this sample is $2.36 \mathrm{~nm} .120$ Figure 4.1-54: Top: micrograph of PCBM/PS Mw=5k Da sample annealed at $145 \mathrm{C} 5 \mathrm{~m}$, bottom left, AFM image from a region between the lateral inhomogeneities; bottom right: GIXD image.Rms roughness measured by AFM is 5.5nm. This is the same sample as in Figure 4.1-51.

Figure 4.1-55: PCBM/PS MW=2k Da sample annealed in-situ over a range of temperatures. Images taken after cooling.

Figure 4.1-56: PCBM/PS MW=2k Da bilayers whose data we have excluded from our analysis. The data plotted here from the samples annealed at $135^{\circ}, 140^{\circ}$ and $145^{\circ} \mathrm{C}$ are not shown in the main body of this chapter, but the double thickness sample data and fit has already been plotted in Figure 4.1-15. Optical micrographs for the $135^{\circ} \mathrm{C}$ and $145^{\circ} \mathrm{C}$ samples are shown in figures 4.1-45 and 4.146.

Figure 4.1-57: PCBM/PS bilayers with MW=2k Da SLD profiles for rejected data due to a fit that didn't capture the fringes of the data. The samples annealed at 135, 140 and 145 are not shown in the main body of this chapter, but the double thickness sample SLD profile has already been plotted in figure 4.1-15. 
Figure 4.1-58: Micrographs for PCBM/PS 2k double (PCBM layer) thickness 125C 5m sample. Top left: a smooth (uniform) area, top right: intermediate area, bottom: the significant presence of inhomogeneities. Out of 30 images chosen from different locations across the entire sample (chosen in an unbiased way by moving the microscope stage without looking at the image), 17 were covered inhomogeneities similar to the level shown in the bottom image, 5 were smooth (uniform), and 8 were intermediate).

Figure 4.1-59: Interfacial roughness for PCBM/PS bilayers fitted with a constant average SLD of top

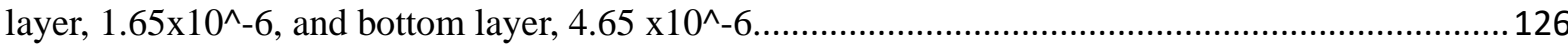

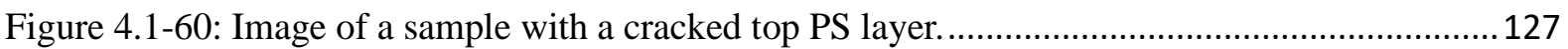

Figure 4.1-61: This figure shows the reflectivity before and after subtraction of the reflectivity from a PCBM single layer for 4 sample $\mathrm{d}, \mathrm{h}, \mathrm{k}$ and $\mathrm{m}$. These samples were ex-situ annealed for $1 \mathrm{~min}$ at $155^{\circ} \mathrm{C}$ (2k-PS sample d), $1 \mathrm{~min}$ at $155^{\circ} \mathrm{C}$ (5k-PS sample h), 5 mins at $145^{\circ} \mathrm{C}$ (20k-PS sample k) and 5 mins at $155^{\circ} \mathrm{C}$ (20k-PS sample $\mathrm{m}$ ). The subtraction was performed using the percentage PCBM-only area coverage on each sample and the reflectivity data from a PCBM single layer sample of the same thickness. In all four cases the chi-squared was drastically improved.............................................128 Figure 4.2-1: NR curves and fits for bis-PCBM/PS bilayers of various PS MW. This graph shows data

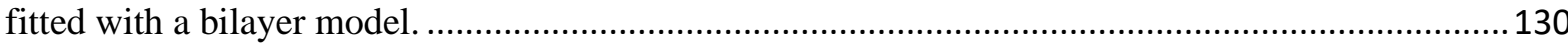

Figure 4.2-2: SLD profiles for bis-PCBM/PS bilayers of various PS MW and unannealed or annealed at various times using a bilayer model.

Figure 4.2-3: bis-PCBM/PS 2k single layer fit: $140 \mathrm{C} 5 \mathrm{~m}$ has a thickness of the single layer as 884 with SLD 2.196 and chisquared=40.4. 145C 5m has a thickness of the single layer as 8603 with SLD

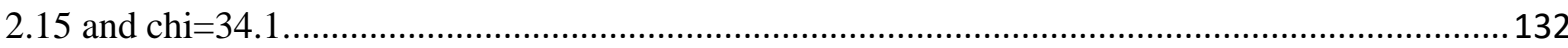

Figure 4.2-4: Multilayer fits for the bis-PSCM/PS samples indicated in the legend........................132 Figure 4.2-5:bis-PCBM/PS samples; multilayer and bilayer SLD profiles. The unannealed profiles are estimated from NR measurements on other (unannealed) samples.

Figure 4.2-6: Multilayer fits of the bis-PCBM/PS bilayers for the molecular weights detailed in the legend.

Figure 4.2-7: This shows the reflectivity curves for a bis-PCBM/PS 100k bilayer showing the full reflectivity curves before and after annealing as well as 30s 'kinetic' measurements taken during annealing showing the gradual change in the reflectivity curve.

Figure 4.2-8: SLD profiles corresponding to the fits before and after in-situ annealing from figure 4.27.

Figure 4.2-9: The change in fit parameters over time for bisPCBM/PS MW=100kDa. Each data point is for reflectivity summed over four 30s measurements barring the inset graphs which show data points for individual 30s measurements. Graph a is thickness of the top layer (PS-rich), graph b is the thickness of the bottom layer (PCBM), graph $\mathrm{c}$ is the SLD of the top layer, $d$ is the SLD of the bottom

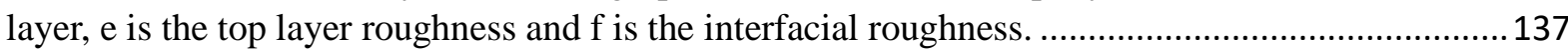

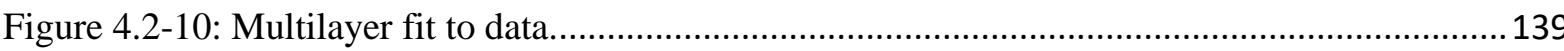

Figure 4.2-11: Comparison of bilayer fit with surface and interfacial roughness to multilayer fit with no roughness

Figure 4.2-12: Interfacial roughness from bilayer fits as a function of PS molecular weight for bisPCBM/PS bilayers. Parameters for the $2 \mathrm{k}$ samples are not included.

Figure 4.2-13: SLD values for the bottom layer as a function of molecular weight (taken from the maximum value of the multilayer fits).

Figure 4.2-14: SLD of the peak of the top layer in initially bis-PCBM/PS bilayers taken from the multilayer fits. The legend indicates the annealing temperature and time.

Figure 4.2-15: The Flory-Huggins free energy of mixing of a PS/bis-PCBM mixture with the lattice size set equal to the size of a bis-PCBM molecule for chi=1.5. The stars indicate experimentally measured compositions 
Figure 4.2-16: Parameter values for fixed and varied oxide layers. The same colour represents the same sample.

Figure 4.2-17: Parameter values for fixed and varied oxide layers. The same colour represents the same sample.

Figure 4.2-18: Micrograph and NR curve for annealed bilayer showing the lateral inhomogeneities on the sample.

Figure 4.2-19: Images of a bis-PCBM/PS MW=100kDa bilayer annealed at 180C 5m. Top left:

micrograph, bottom left: AFM image, bottom right: GIXD image 150 Figure 4.2-20: Micrographs showing variation in area coverage by defects for bis-PCBM/PS 5k 140C $5 \mathrm{~m}$. Top left: a low defect density region, top right: intermediate defect density, bottom: high defect density.

Figure 4.3-1: Reflectivity for two samples measured both prior to annealing and after annealing following storage. Both samples are PCBM/2k-PS.

Figure 4.3-2: This figure shows the SLD profile for samples measured prior to annealing and after annealing three months later (left) and after six weeks (right). Typically the unannealed samples shown were measured within a week of fabrication.

Figure 4.3-3: NR curves and fits for PCBM/PS samples aged under various conditions or made using oxidised PCBM. Bilayer fits with Gaussian interface roughness.

Figure 4.3-4: SLD bilayer profiles for PCBM/PS samples aged in various conditions or using oxidised PCBM and annealed at various times and using various molecular weight polystyrene (2k and 20k $\mathrm{Da})$.

Figure 4.3-5: NR curves and fits for samples whose PCBM layer was oligomerised and deoligomerised to varying extents by thermal annealing before deposition of the top PS layer with varying PS MW using a bilayer model. $40 \%$ was maximally oligomerised and not annealed prior to deposition of top layer, $25 \%$ was annealed at $110^{\circ} \mathrm{C} 1 \mathrm{hr}, 10 \%$ was annealed at $130^{\circ} \mathrm{C} 1 \mathrm{hr}$, fully deoligomerised was annealed at $160^{\circ} \mathrm{C} 1 \mathrm{hr}$. Following deposition of top layer all samples were annealed at $135^{\circ} \mathrm{C}$ for $5 \mathrm{mins}$

Figure 4.3-6: SLD profiles for samples whose PCBM layer was oligomerised and de-oligomerised to varying extents by thermal annealing before deposition of the top PS layer with varying PS MW...158 Figure 4.3-7: Multilayer fits with no roughness between the layers.

Figure 4.3-8: Comparison of bilayer fit with roughness between the layers and multilayer fit with no roughness between the layers. The unannealed data is estimated from NR measurements on unannealed bilayers

Figure 4.3-9: Interfacial roughness from bilayer fits as a function of molecular weight for samples deliberately aged variously in light, dark, air and under nitrogen. The samples aged in light was for one week at 800 lux in air and in the dark for 6 weeks

Figure 4.3-10: Top layer SLD as a function of molecular weight for samples deliberately aged variously in light, dark, air and under nitrogen. The samples aged in light was for one week at 800 lux in air, and in the dark for 6 weeks.

Figure 4.3-11: Parameter values for fixed and varied oxide layers. The same colour represents the same sample.

Figure 4.3-12: Parameter values for fixed and varied oxide layers. The same colour represents the same sample.

Figure 4.3-13: Micrograph and NR curve for annealed bilayer showing the lateral inhomogeneities on the sample.

Figure 4.4-1: Reflectivity curves and fits for single layer blend (initially a bilayer) of PCBM and

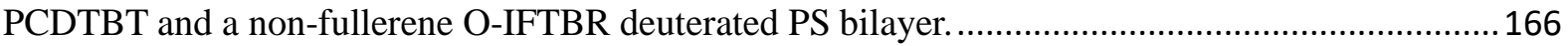

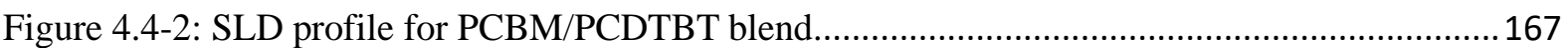
Figure 4.4-3: O-IDFBR/dPS bilayer SLD annealed above the glass transition.................................168 
Figure 4.5-1: Growth of needles and fans for 25nm PCBM, PS 300k 40nm 180C (top) and PCBM $30 \mathrm{~nm}$, PS 20k 30nm 210C (bottom).

Figure 4.5-2: OM images of samples annealed at 170C for PCBM/PS bilayers with MW=20k with the

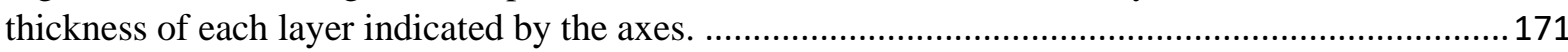
Figure 4.5-3: OM images of samples annealed at 180C for PCBM/PS bilayers with MW=20k with the

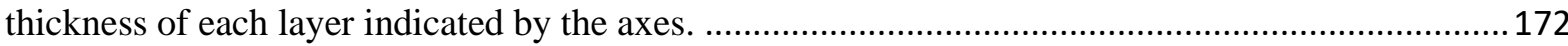

Figure 4.5-4: OM images of samples annealed at 170C for PCBM/PS bilayers with MW=300k with

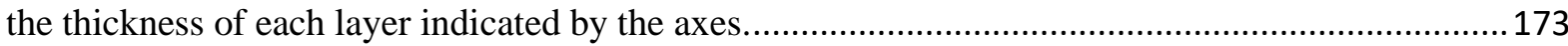
Figure 4.5-5:: OM images of samples annealed at 180C for PCBM/PS bilayers with MW=300k with

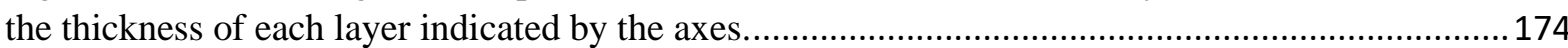
Figure 4.5-6: Categorisation of observed morphologies for the preceding OM images.....................175 Figure 4.5-7: Categorisation of observed morphologies for the preceding OM images.....................175 Figure 4.5-8: Categorisation of observed morphologies for the preceding OM images.....................175 Figure 4.5-9: Categorisation of observed morphologies for the preceding OM images.....................176 Figure 4.5-10: OM images of fans for samples annealed at different temperatures showing a decrease in nucleation density and increased dimensions with increasing temperature. Data are shown for the PCBM and PS thicknesses that are both $30 \mathrm{~nm}$ with PS MW of $20 \mathrm{k}$.

Figure 4.5-11: AFM images showing morphology development for fans annealed at different temperatures for different times (until crystal growth slowed or was impeded). The size of the scan is shown on the axis. PCBM and PS thickness es are both $30 \mathrm{~nm}$. The PS MW is $20 \mathrm{k}$ 179 Figure 4.5-12: A profile of a fan taken normal to the growth direction indicating the depletion zones to either side of the crystal. The red line shows a stem width from trough to trough on either side of the crystal.PS 20k 30nm PCBM 30nm 150C

Figure 4.5-13: Stem width measured by AFM for different annealing temperatures. Values averaged over multiple crystals.

Figure 4.5-14: 30nm PS and 30nm PCBM film annealed at $150^{\circ} \mathrm{C}$ showing distinct branching of the crystals.

Figure 4.5-15: 30nm 20k PS and 30nm PCBM film annealed at $190^{\circ} \mathrm{C}$ showing dinstinct branching of the fibrils within thicker outer arms.

Figure 4.5-16: $30 \mathrm{~nm}$ PS and 30nm PCBM film annealed at $230^{\circ} \mathrm{C}$ showing isolated branches with a broad width.

Figure 4.5-17: Surface profile for the line shown in figure 4.5-16 182 Figure 4.5-18: $30 \mathrm{~nm}$ PS and 30nm PCBM film annealed at $230^{\circ} \mathrm{C}$ showing isolated branches with a broad width. This is the same crystal that is shown in figure 4.5-15. 182 Figure 4.5-19: Surface profile for slice indicated in figure 4.5-18 183 Figure 4.5-20: Crystal length measured on single crystals for progressive annealing times (measured by optical microscopy). Layer thicknesses and MW shown in legend.

Figure 4.5-21: Crystal length (measured by optical microscopy) for unimpeded crystals at different temperatures for layer thicknesses and MW shown in legend.

\section{List of Tables}

Table 1: Setpoint and sample surface temperature for in-situ annealed samples.

Table 2: Setpoint and sample surface temperature for ex-situ annealed samples in the vacuum oven. 67 
Table 3:fit parameters for two PCBM/PS MW=5k bilayers made and annealed under the same protocol $(145 \mathrm{C} 5 \mathrm{~m})$ and measured at the Figaro reflectometer at the ILL and the D17 reflectometer at ISIS.

Table 4: The fitted parameter values for two separate samples measured at two separate detectors but made and annealed with the same protocol; PCBM/PS MW=20k Da 145C 5m.

Table 5: The six fit parameter values for the same PCBM/PS MW=5k Da sample (annealed at $135{ }^{\circ} \mathrm{C}$ for 5 minutes) measured twice, once at INTER detector at ISIS and once at the D17 detector at the ILL.

Table 6:Comparison of parameters for different thicknesses (all annealed for 5mins). These are the thickest samples shown in Figure 4.1-17 and Figure 4.1-18 below.

Table 7: List of parameter values for PCBM/PS MW=2k samples with a fixed or varied oxide layer.for the samples in Figure 4.1-4.....

Table 8: A list of which samples were measured at which reflectometers where all samples are PCBM/PS bilayers unless otherwise indicated.

Table 9: List of measured thickness for different spin speeds, concentrations and molecular weights measured by AFM compared with unannealed values measured by NR

Table 10: Values obtained from ImageJ of the coverage of the top PS layer on the relevant samples.

Table 11:List of where the stated samples were measured 


\section{Introduction}

\subsection{Thesis Motivation and Outline}

In this thesis, the motivation is to improve the understanding of polymer-fullerene thin-film compositions with applications in OPV technology. The aim is to complement OPV device optimisation work by examining the fundamental behaviour of polymer nanocomposite systems. Complex behaviour has been reported for OPV thin films, so simplified geometries and materials are used. The effect of MW on mixing and interfacial width in model polymer/fullerene bilayer systems is explored, as well as the effect of layer thickness on crystal morphology. To probe the depth profile, specular neutron reflectivity is used to characterise the mixing of the materials within a bilayer geometry, as well as the interfacial roughness between the two layers. In almost all samples (barring two exceptions) the initial composition profile consisted of a bilayer of pure materials. Following annealing there is diffusion between the layers, causing a change in the layer thicknesses; however a bilayer structure is preserved. There is an an in-depth investigation of the hypothesis that during annealing the two materials reach a liquidliquid equilibrium. This hypothesis is robustly examined in both the PCBM/PS and bis-PCBM/PS systems, by looking at the composition of the proposed co-existing phases in the two layers, and the interfacial width, as a function of MW, annealing time, annealing temperature and layer thickness.

Flory-Huggins theory and self-consistent-field-theory (SCFT) are applied to compare predictions with experiment. Optical microscopy and AFM are used to assess sample quality with-respect-to the neutron reflectivity samples, and also to identify the formation of micron-sized crystals (that occur at higher temperatures).

The structure of the thesis is as follows; The current chapter (chapter 1) deals with the background of OPV materials, particularly relating to polymer/fullerene systems. Chapter 2 describes the equilibrium theories of polymers, polymer mixtures and polymer interfaces. Chapter 3 details the materials and experimental methods used, and the theory associated with these methods. Chapter 4 describes the results obtained on PCBM/PS and bis-PCBM/PS bilayers, respectively, and discusses these 
results in the light of theory. This includes a discussion of the overall conclusions for both systems at the end of Chapter 4.2. All of the results in Chapter 4 are for samples that were prepared and annealed in the dark, but Chapter 4.3 looks at PCBM/PS samples that that were illuninated by visible light under various conditions, and examines the effects of this on the mixing behaviour, compared to non-illuminated samples. Chapter 4.4 is a very brief chapter in which preliminary data is presented on mixing in polymer/small-molecule bilayers containing either an amorphous conjugated polymer or a non-fullerene (small molecule) acceptor. Chapter 4.5 looks at the morphology of large PCBM crystals in bilayers that are annealed over a wide range of temperatures (mostly at significantly higher temperatures than used in Chapters 4.1 - 4.3). Chapter 5 details the conclusions and potential future work.

\subsection{Organic Photovoltaic Materials}

One of the biggest challenges facing society today is to move away from fossil fuels and generate renewable energy. One candidate for this energy generation is the sun. If all the energy incident on the Earth from the Sun was captured for one hour it would provide for all of humanity's needs for one year. Solar energy is the conversion of the Sun's radiation into electricity. The photovoltaic effect is one way to capture this radiation, where the photons from the Sun excite electrons in a material leading to a voltage across the material. Many materials exhibit the photovoltaic effect. Silicon has a bandgap in the visible part of the spectrum and exhibits this effect. For this reason silicon is used as a photovoltaic material and it is abundant in the Earth's crust. However, silicon is costly to produce. Some organic materials, such as polymers and fullerenes, also exhibit the photovoltaic effect. Research and development into organic photovoltaics (OPVs) is an established field that has shown a steadily increasing power conversion efficiency (PCE) over 30 years (Spanggaard and Krebs 2004). The advantages of OPVs are the ease of solution-processing and the low cost of the materials relative to the cost of silicon. Silicon forms the active element of the solar cells that currently dominate the commercial market. 


\section{The basis for developing OPVs}

The challenges facing the OPV community are to increase the PCE (by using conjugated materials, such as polymers with suitable bandgaps) and to extend the lifetime of the OPV cells. Conjugation refers to the pi orbital electrons being delocalised within the molecules, for example along a polymer backbone due to the presence of alternating single and double carbon-carbon bonds as in Figure 1.2-1.

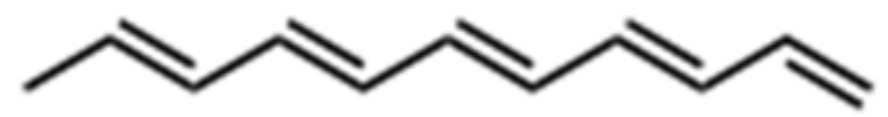

Figure 1.2-1: Schematic of the conjugated polymer, polyacetylene, showing the alternating single and double bonds.

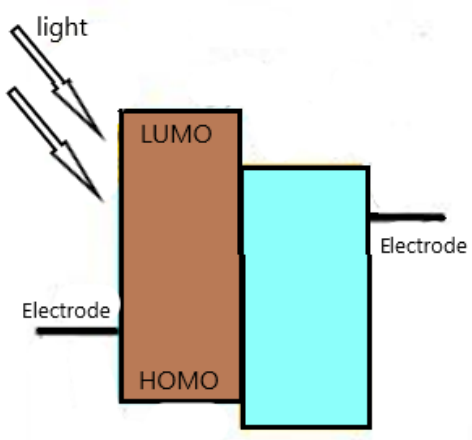

Figure 1.2-2: Schematic a heterojunction between electron donating (brown) and electron accepting (blue) materials, within OPV devices. HOMO is the highest occupied molecular orbital, and LUMO is lowest unoccupied molecular orbital. Charge generation occurs when an electron moves fro the HOMO to the LUMO to the acceptor to the electrode and is separated from the hole that moves from the HOMO to the other electrode.

This conjugation can lead to semiconducting or metallic-like behaviour and also enables visible light absorption and the photovoltaic effect. The photovoltaic effect is the creation of an electrical current from the incidence of photons. A 'heterojunction' can be formed at the interface between two semiconducting materials, which means that there is an offset in their bandgaps, as shown in Figure 1.2-2.

In 1986 an all polymer photovoltaic cell was designed and reached efficiencies of $1 \%$. Introducing the fullerene $\mathrm{C} 60$ as an electron acceptor increased efficiency by one order of magnitude when first introduced (Spanggaard and Krebs 2004). 
Subsequently, efficiency was increased further by use of a blend rather than a bilayer (Spanggaard and Krebs 2004).

By attaching a short organic side-chain to the C60 cage, the molecule becomes more compatible with an organic solvent and more easily dissolved. This allows solutionprocessing. While many fullerenes are being investigated, the most common one used today is phenyl-C60-butyric acid methyl ester (PCBM). PCBM is the fullerene used for this thesis, along with bis-adduct- phenyl-C60-butyric acid methyl ester (bis-PCBM) which contains two (identical) side chains. This inhibits crystallisation as the two side chains can be located at different positions on the C60 cage in each molecule. This uneven distribution makes regular packing very difficult.

The active layer in an OPV must be sandwiched between two planar electrodes, one by necessity must be transparent to allow the sunlight to impinge on the photoconducting materials in the active layer. The most common choice is to use the transparent semiconductor indium-tin-oxide (ITO) deposited onto a glass substrate, although flexible substrates (Figure 1.2-3) are being developed.

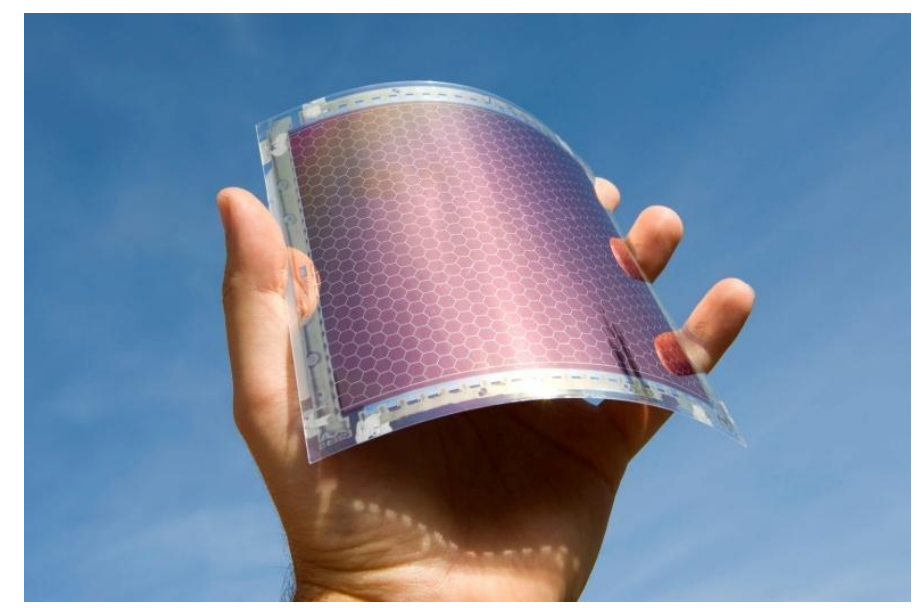

Figure 1.2-3: A flexible OPV module reproduced under the Creative Commons licence from reference (Galagan and Andriessen 2012).

When light is incident on the active layer, an exciton is formed. An exciton is a quasi-particle made up of an electron and hole that are Coulombically bound. In conjugated polymer/fullerene systems the polymer is usually the electron donor and the fullerene the acceptor. If the light was absorbed in the electron donor, the exciton moves along a polymer backbone, or from one polymer molecule to another. Once it meets the donor-acceptor interface, the charges can be separated, and then travel towards their respective electrodes. It has been found that the exciton can only move 
a distance of 10nm before the electron and hole recombine (Van Bavel et al. 2009). Because of this, the morphology of the active layer is of crucial importance in maximising the amount of charge extracted. Larger interfacial area increases charge separation but can lead to a more complex path for charge transport to the electrodes. Finding the optimal structure is not straightforward as different polymers and fullerenes have different miscibilities and dispositions to form crystals when annealed. It is important to understand the underlying physics which might be applied to different polymer/fullerene systems.

The bulk heterojunction (BHJ) is the most effective architecture for obtaining a bicontinuous network of donor and acceptor. It is comprised of an intermixed blend of the donor and acceptor material as in Figure 1.2-4. BHJs can be made by spincoating a blend solution or by annealing a bilayer of two miscible species (D. Chen et al. 2011). While BHJs are more efficient than bilayers, because of the greatly increased interfacial area, they have a complex morphology (Carrillo et al. 2013). Layered structures can be used to simplify the geometry. PCBM and the conjugated polymer poly (3-hexylthiophene) (P3HT) are probably the most used material pair in OPVs. Sequential spin-coating of PCBM onto P3HT causes significant mixing of the two materials, (K. H. Lee et al. 2011), and therefore a number of studies have employed film floating and other techniques to fabricate bilayers and study mixing in this system, starting from two pure layers (D. Chen et al. 2011; H. Chen et al. 2012; Ro et al. 2012). These studies have found considerable miscibility between PCBM and amorphous P3HT. However, this system has two substantial drawbacks in terms of trying to understand the fundamental behaviour of polymer/fullerene mixing; firstly, both components can crystallise in this system (with spin-coated P3HT layers showing extensive crystallisation), and secondly there is poor control of the molecular weight distribution in conjugated polymers, which hampers the study of MW-dependent behaviour (the particular focus of this thesis). This thesis uses model bilayers, consisting of fullerenes and the well-controlled (low polydispersity) polymer, atactic polystyrene, to study the mixing compositions of two originally pure domains upon annealing. 


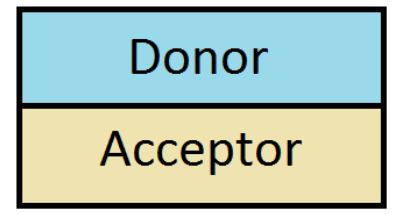

Bilayer

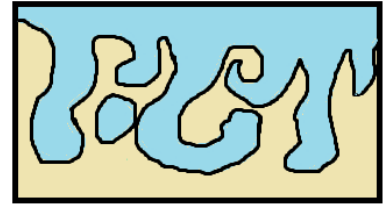

$\mathrm{BHJ}$

Figure 1.2-4: Left shows a bilayer architecture while right shows a BHJ.

\section{Technical challenges of OPVs}

The materials used in devices are conjugated polymers, specifically which ones to use is an active question. In the early days of plastic solar cells polyphenylene vinylene (PPV) based materials were used, but the bandgaps were too large to reach efficiencies above 3\% (Spanggaard and Krebs 2004). The performance was boosted by use of polythiophenes such as poly[3- hexylthiophene-2,5-diyl] (P3HT). Poly[N90-heptadecanyl-2,7-carbazole-alt-5,5-(40,70-di-2-thienyl-20,10,30benzothiadiazole)] (PCDTBT) is a more recently employed polymer which has achieved PCE of 7.5\% (Ragoussi and Torres 2015). PCDTBT has been shown to be stable when tested under accelerated conditions for an estimated seven years (Y. Zhang et al. 2016) making it a promising candidate for future developments. Nonfullerene acceptors (NFAs) are also being developed with PCE already at 10\% (Eftaiha et al. 2014; Baran et al. 2016).

The molecular weight of the polymer can have a complex effect on efficiency. Higher molecular weight can lead to a sharper molecular interface between two components in a polymer-polymer system (see discussion in section 2.6 below) which can improve charge separation (Kuang, Janik, and Gomez 2015). However, higher molecular weights can also decrease charge mobility (Ballantyne et al. 2008). The interfacial width and the extent of mixing can be theoretically described for liquid polymer-polymer mixtures by mean-field theories (Adhikari 2012). These theories and the extent to which they are applicable in polymeric and small-molecule systems are discussed in section 2.6 below.

The PCE is limited theoretically by the Shockeley-Queisser (S-Q) Limit which for an optimum band gap of $1.34 \mathrm{eV}$ is $33.7 \%$. PVs that utilise silicon as the 
semiconductor are near to this limit (mono-crystalline Si cells at 26.7\%) while OPVs are currently at a maximum PCE of $11.2 \%$ (Green et al. 2018) . A detailed description of the S-Q Limit is described in the reference (Polman et al. 2016).

Aside from PCE the other critical factor in enabling OPVs to compete with current commercial PVs is the lifetime of the cell. It has been shown that lifetimes can be on the order of years (Teran-Escobar et al. 2012). However, silicon-based solar cells have lifetimes on the order of tens of years.

The PCE degrades with exposure to oxygen and water as these cause chemical reactions that alter the materials present in the active layer and even the surfaces of the electrodes. Also, even within an inert atmosphere, PCBM is sensitive to light which causes oligomers of the fullerene to form (Wong et al. 2014). Oligomerisation has been shown to be reversible ( $\mathrm{Z}$. Li et al. 2013) by annealing (under an inert atmosphere). There also occurs irreversible aspects of the degradation in the formation of carbonyl and carboxylic groups which act as traps to the charges (Seemann et al. 2011). Studies have found that degradation can be initiated at the electrodes with the formation of oxide layers at the electrodes or chemical bonds within the active layer materials (the BHJ) (Kumar et al. 2016). There is also significant mobility of polymers in thin films at temperatures below the bulk glass transition for low MW, as discussed in section 2.3 (Keddie, Jones, and Cory 1994; Santangelo and Roland 1998). This mobility can cause the morphological or compositional evolution of the active layer during operation (Campoy-Quiles et al. 2009). With conjugated polymers, there is often a high polydispersity (Spoltore et al. 2015), so MW effects are likely to be significant, even when using high nominal MW.

\section{PCBM Crystallisation}

PCBM can crystallise when heated above the glass transition (Y. Yang et al. 2014). Various crystal morphologies are observed depending on the substrate, solvent and the temperature (G. Li et al. 2008; Dang, Hirsch, and Wantz 2011; Volonakis, Tsetseris, and Logothetidis 2012). Two forms of PCBM crystal are reported in the literature: micron-sized crystals (needles or branched crystals; see Figure 1.2-5 and Figure 1.2-6) and nano-scale crystals (Yang et al. 2004; H. H. Lee et al. 2013; Môn et al. 2015). Micron-sized crystals can rupture the carefully developed BHJ 
morphology, drastically reduce the interfacial (heterojunction) area between the donor and acceptor, and decrease the PCE of a cell (Woo et al. 2008).

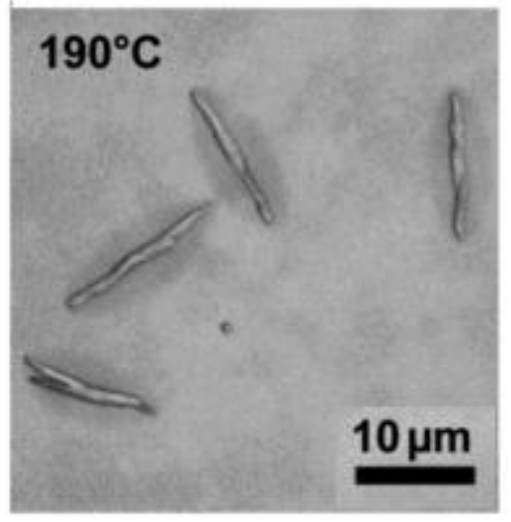

Figure 1.2-5: Needle-like micron-sized crystals on a glass substrate for 1:1 blend of polymer and $\mathrm{PCBM}$ annealed at $190^{\circ} \mathrm{C}$ reproduced with permission from reference(Lindqvist et al. 2013)

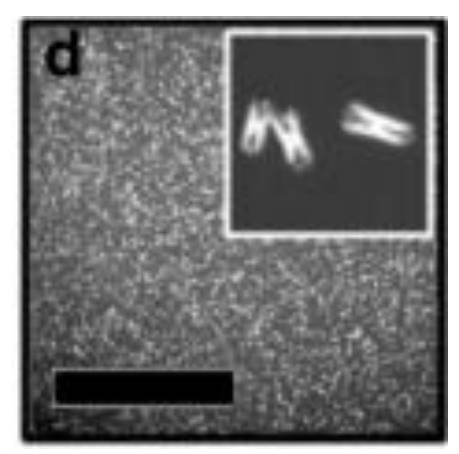

Figure 1.2-6: Branched crystals formed when PCBM:PCDTBT 2:1 blend annealed at 140 ${ }^{\circ} \mathrm{C}$ for $1 \mathrm{hr}$ in the dark. Scale bar is $100 \mu \mathrm{m}$, and inset optical image is 20x20um. Reproduced with permission from reference (Z. Li et al. 2013)

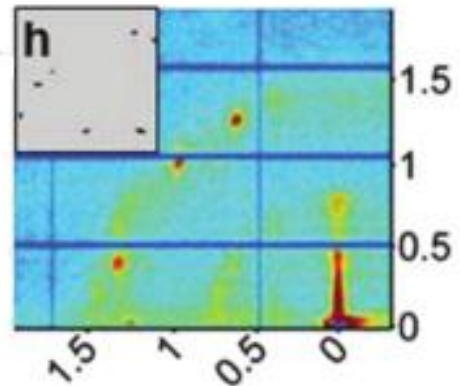

$q_{x y}\left(\AA^{-1}\right)$

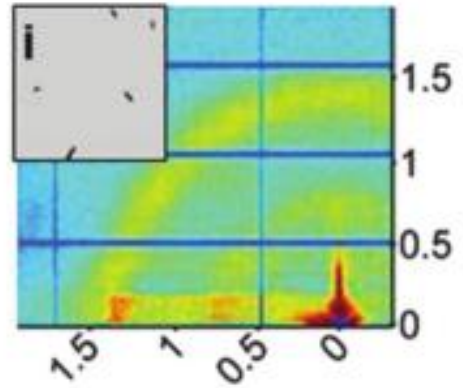

$q_{x y\left(\AA^{-1}\right)}$

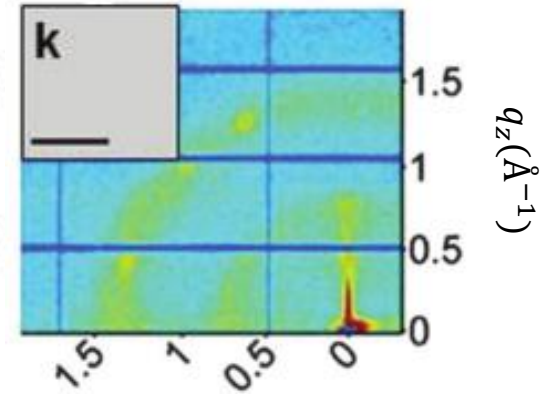

$q_{x y}\left(\AA^{-1}\right)$

Figure 1.2-7: GIXD patterns and optical micrographs (insets) of two PCBM/PS bilayers and a PCBM single layer. The PCBM/PS bilayers were annealed at $170{ }^{\circ} \mathrm{C}$ for 20 minutes ( $h$ and $i$ ) and the PCBM single layer $(k)$ was annealed for 60 minutes. The PS thicknesses were as follows; h) 8nm, i) $25 \mathrm{~nm}$ PS. All samples had a PCBM layer thickness 20nm (before annealing). The PS MW was 350kDa in all samples. Reproduced with permission from reference (Môn et al. 2015). The scale bar in k) is $20 \mu \mathrm{m}$, and all micrographs are the same magnification. The GIXD patterns are detector maps with the scales indicating $q_{z}$ and $q_{x-y}\left(\right.$ in units of $\left.\AA^{-1}\right)$ as defined in Môn et al (Reproduced under the Creative Commons licence from Môn et al 2015).

Mon et al.( Môn et al 2015) found evidence for both nano-scale and micron-sized PCBM crystals in annealed PCBM/PS bilayers (the PS layer on top), with the growth 
of both kinds of crystal strongly dependent on the thickness of the PS layer (for thicknesses below around $30 \mathrm{~nm}$ ).

Figure 1.2-7 shows grazing-incidence $x$-ray diffraction (GIXD) patterns from two bilayers (labelled h and i) and also an annealed PCBM single layer (labelled k).

Figure 1.2-7 k shows the presence of crystallisation (characteristic Bragg peaks, due to PCBM crystals that have been seen by previous workers (Verploegen et al. 2010; Hopkinson et al. 2011)) that is not visible by optical microscopy, as the inset image is an optical micrograph showing no features on the sample surface while the GIXD pattern shows Bragg peaks indicative of extensive crystalline ordering.

Figure 1.2-7 $\mathrm{h}$ and i show similar levels of coverage by micron-sized PCBM crystals to one another (observable via optical microscopy as in the inset image), but very different GIXD patterns. The Bragg peaks seen in

Figure 1.2-7 $\mathrm{h}$ and i suggest the presence of significant nano-scale crystals (as opposed to the micron sized crystals visible by optical microscopy) for the thinner (8nm) PS layer sample, in comparison to the thicker (25nm) PS layer sample (which just shows a ring-like scattering pattern from amorphous PCBM). Mon et al. proposed a mechanism for the growth rate of both micron-sized needles and nanocrystals, involving the interaction between these processes, as a function of PS film thickness. Crystal growth-rates are not examined in this thesis, but (as discussed in later chapters) the amount of crystallisation present in the samples is carefully examined.

Crystallisation, mixing and interfacial width all play a role in device performance. Mon et al. found that there was rapid diffusion of some PCBM into the PS layer in a model PCBM/PS bilayer system using NR. They also found that this diffusion occurred before significant crystallisation and hypothesised that for short annealing times ( $<5-10$ minutes) this led to a liquid-liquid equilibrium. The majority of the work presented in this thesis looks at the development of the composition profile within this same system (and the closely related bis-PCBM/PS) as a function of annealing time, temperature and PS MW, with the aim of testing this equilibrium hypothesis. Crystallisation of PCBM was monitored and minimised during these studies. 


\section{Background}

\subsection{Introduction to Polymers}

The following sections outline standard polymer theory and a complete description can be found in Soft Condensed Matter by Jones (R. A. L. Jones 2002), Polymer Physics by Rubinstein and Colby (R. Colby; M. Rubinstein; 2003) and Polymers at Surfaces and Interfaces by Jones and Richards (R. A. L. Jones and Richards, R. W. 1999).

A polymer is a chain of molecules, each called a monomer, connected along a backbone. Each unit is repeated multiple times and the number of times is called the degree of polymerisation, $N$. This number is typically very large, and can be on the order of $10^{4}-10^{5}$. The molar mass, $M$, of a polymer, is

$$
M=N M_{\text {mon }},
$$

where $M_{m o n}$ is the molar mass of the polymer's chemical monomer.. The atomic arrangement within individual polymer chains is very important in determining the macroscopic qualities, for example, crystallisation. A chain of randomly oriented monomers will be less likely to crystallise as it cannot pack regularly. Different configurations of the same chemical monomers are called isomers. There are three categories of isomers: sequence, structural and stereo.

Sequence isomerism is when the monomers are joined together in different orientations when the monomer has two end groups, and these can be joined either head to head or head to tail(R. Colby; M. Rubinstein; 2003).

Structural isomerism is if the polymer has a double bond in its backbone, and the groups cannot rotate, leading to distinct structures for the same combination of monomer. The third type of isomer is a stereoisomer in which the groups attached to the chain are arranged on the same or opposite sides of the chain, defined by tacticity. If all the groups are on the same side it is known as isotactic, if they alternate regularly, they are known as syndiotactic, and if they are randomly arranged, it is known as atactic.

Polymers can be found in nature or synthesised. When they are synthesised there is always a spread in the distribution of molecular weight, which is known as the 
polydispersity. If all the polymers have the same number of monomers, it is said to be monodisperse.

The number fraction, $n_{N}$, is the fraction of polymers present containing $\mathrm{N}$ monomers. The distribution is this plotted against the molar mass of the of the molecules, $M_{N}=$ $N M_{\text {mon }}$. Two definitions are typically used: weight average and number average molar mass. These are found from taking ratios of moments of the number fraction distribution. The kth moment is defined as

$$
m_{k}=\sum_{N} n_{N} M_{N}{ }^{k} .
$$

The number average molar mass, $M_{n}$, is defined as the ratio of the first to zeroth moments of the distribution as

$$
M_{n}=\frac{m_{1}}{m_{0}}=\sum_{N} n_{N} M_{N}
$$

as $m_{0}=1$ (the distribution is normalised). The weight average molar mass, $M_{W}$, is defined as the ratio of the second and the first moments:

$$
M_{W}=\frac{m_{2}}{m_{1}}=\frac{\sum_{N} n_{N} M_{N}^{2}}{M_{n}}
$$

The polydispersity of a polymer is the ratio of these two: $M_{W} / M_{n}$. The degree of polymerisation affects the physical properties such as glass transition temperature, boiling point. and melting point(R. Colby; M. Rubinstein; 2003). These values typically rise with increased $N$.

\subsubsection{Ideal polymer chains: Polymer random walks}

To define a measure of extension or length for a polymer molecule, the different possible configurations must be taken into account. The polymer can have freely rotating bonds or be stiff with a more restricted range of motion. For a freely jointed polymer molecule of $N+1$ backbone atoms the end-to-end vector, $R$, is the sum of the length of each backbone atom, $r$ :

$$
R=\sum_{i}^{N} r_{i}
$$


As all of the possible conformations are isotropic, the average possible end-to-end distance assumed by the polymer by $\langle\boldsymbol{R}\rangle$ is zero. Instead, the root mean squared, $\sqrt{\left.<R^{2}\right\rangle}$, can be found:

$$
\left\langle R^{2 .}\right\rangle=\left\langle\sum_{i, j}^{N} r_{i} \cdot r_{j}\right\rangle=N l^{2}+\left\langle\sum_{i \neq j}^{N} r_{i} \cdot r_{j}\right\rangle=N l^{2},
$$

where $l$ is the unit length of a monomer. Equation (2.6) is found using $r_{i} \cdot r_{j}=l^{2} \cos \theta$. For $\mathrm{i}=\mathrm{j}, \cos \theta=1$ and for $\mathrm{i} \neq \mathrm{j}$, the mean value of $\cos \theta=0$. In other words, the root mean square end to end distance of a polymer chain of $\mathrm{N}$ steps is the root of the number of steps times the monomer length.

In the freely rotating chain model, the bonds have fixed bond angle, $\theta_{i}$, and the cross terms in equation (2.6) become

$$
\left\langle r_{i} \cdot r_{i-m}\right\rangle=r^{2} \cos ^{m} \theta
$$

As $m$ gets large, $\cos ^{m} \theta$ approaches zero, i.e., the correlations die out along the chain. Using this fact, the chain can be treated as consisting of subunits that are larger than the distance over which the correlations die away, say g steps (chemical monomers) in each new subunit. The chain can now be described as a random walk of N/g new subunits, with vector $d_{i}$ representing subunit $i$. The root mean squared end-to-end distance can then be written as

$$
\left\langle r^{2}\right\rangle=\frac{N}{g}\left\langle d^{2}\right\rangle=N_{b} b^{2} .
$$

Here $b$ is known as the statistical step length, which is a measure of chain stiffness and the number of subunits is redefined as $N_{b}$ so that the end to end distance is the same. An alternative description of a polymer chain undergoing a random walk can be formulated by defining a Kuhn monomer of length $l_{k}$, such that the mean-squared end-to-end distance is equal to $N_{k} l_{k}^{2}$ and the contour length is equal to $N_{k} l_{k}$; i.e. the chain consists of a freely jointed chain of $N_{k}$ Kuhn monomers of length $l_{\mathrm{k}}$. The Kuhn monomer length is equal to twice the persistence length, defined in the worm-like chain model (which is not discussed here) (Saeki 1997).

Returning to the freely-jointed chain model, for large $N$ the probability distribution, $\mathrm{P}$, of different end-to-end vectors is Gaussian and is given by 


$$
P(r, N)=\left(\frac{2 \pi N l^{2}}{3}\right)^{-3 / 2} \exp \left(\frac{-3 r^{2}}{2 N l^{2}}\right)
$$

From this equation the configurational entropy (R. A. L. Jones 2002), S(r) can be written as

$$
S(r)=-k_{B} \sum_{i=1}^{N} P_{i} \ln \left(P_{i}\right)=-\frac{3 k_{B} r^{2}}{2 N l^{2}}+\text { constant }
$$

\subsection{Introduction to Fullerenes}

Fullerenes were discovered by Croto, Curl and Smalley in 1985, winning them the Nobel prize in chemistry. They have truncated icosahedrons in the form of a cage of carbon atoms. There are several such shapes which are stable, with the most stable and common being $C_{60}$, which has 12 pentagons and 20 hexagons as in Figure 2.2-1. The carbon cage is around $7 \AA$ in diameter (Thakral and Mehta 2006).

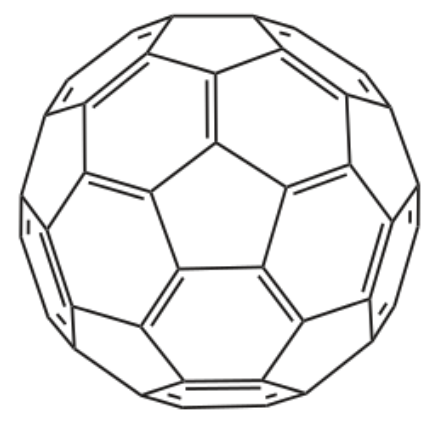

Figure 2.2-1: The fullerene C60.

Fullerenes are highly symmetrical with 120 symmetry operations. Due to the delocalised pi electrons, fullerenes have excellent electronic properties and are a popular choice in OPV devices as the electron acceptor in the active layer.

It is possible to functionalise a fullerene, to make it more soluble, for example, and popular choices of such fullerene derivatives are pheny-C61-butyric acid methyl ester (PCBM) and bis-adduct PCBM (both shown in Figure 2.2-2) which has an extra side-chain that inhibits crystallisation, most likely due to variability in the relative locations of the two side-chains and therefore a lack of regular structure of the molecules. 

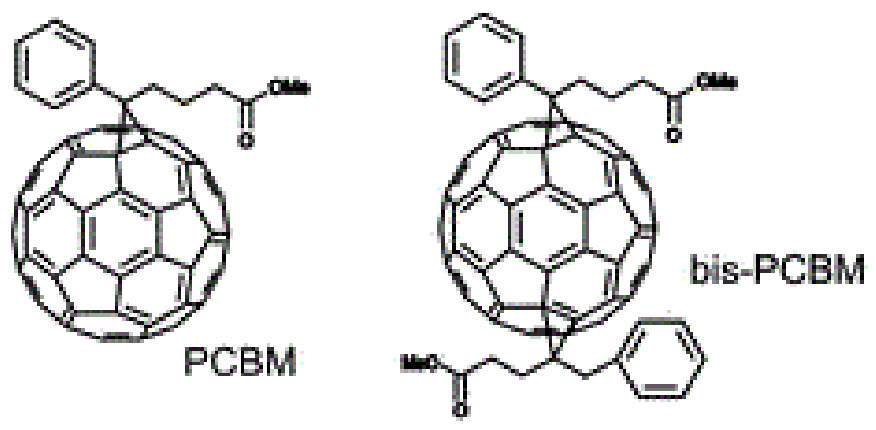

Figure 2.2-2: Chemical structure of PCBM and bis-PCBM reproduced with permission from reference (Brabec et al. 2010)

Because of their low lying lowest unoccupied molecular orbital (LUMO) levels fullerenes act as electron acceptors in OPVs. Theoretically, they might also be used as donors as demonstrated by Zhuang et al. (Zhuang et al. 2013) when they fabricated all fullerene OPV devices. C60 was used in the original discovery of charge transport from polymer to fullerene in the 1990s, but since then the prototypical choice has become PCBM due to increased solubility (Ferguson, Blackburn, and Kopidakis 2013). The light absorption wavelength of PCBM peaks around 300nm.

Fullerenes have applications in OPVs, antioxidants, biopharmaceuticals, catalysts, water purification and medicine. The glass transition of PCBM, which is used in this project, is reported as $131.2^{\circ} \mathrm{C}$ (J. Zhao et al. 2009).

PCBM can oxidise when exposed to light and air (Emily M. Speller et al. 2017). The oxidation effect can be observed for illumination times of less than 40 minutes under a solar spectrum. 


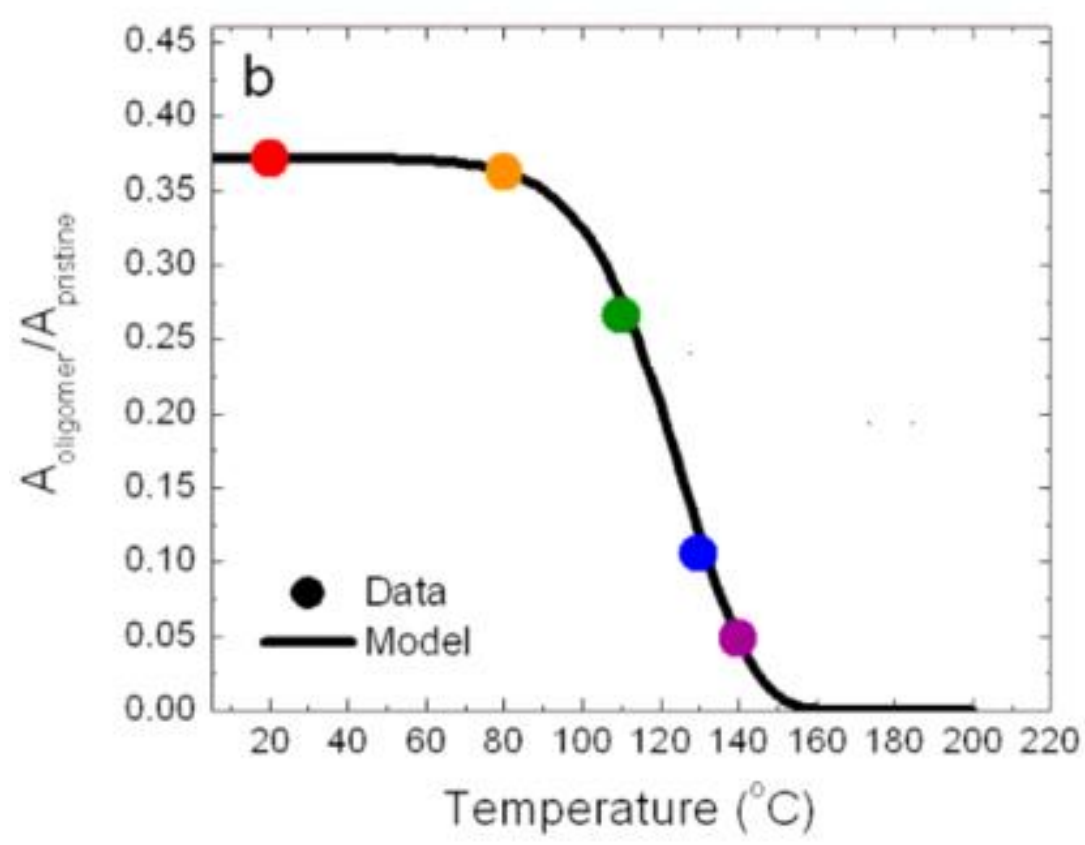

Figure 2.2-3:The effect of heat treatment on the extent of oligomerisation in PCBM films. The PCBM films were first oligomerised to a maximal 37\%, by illumination with visible light in an inert atmosphere. The films were then subjected to an hour of annealing at the temperatures shown. Reprinted (adapted) with permission from (Wong, Him Cheng, Zhe Li, Ching Hong Tan, Hongliang Zhong, Zhenggang Huang, Hugo Bronstein, Iain McCulloch, João T. Cabral, and James R. Durrant. 2014. "Morphological Stability and Performance of Polymer-Fullerene Solar Cells under Thermal Stress: The Impact of Photoinduced PC60BM Oligomerization." ACS Nano 8 (2): 1297-1308. doi:10.1021/nn404687s.). Copyright (2014) American Chemical Society. The y-axis shows the number fraction of oligomers with-respect-to the total amount of PCBM. The fractions were taken from gel permeation chromatographic analyses comparing the size of the signal from oligomerised PCBM to pristine PCBM.

In the experiments described in chapter 4.3 the oxidation was performed on unannealed bilayers or pure solution of PCBM, so the effect of the PS would not be relevant. The effect of oxidation on an OPV device is a reduction in the efficiency due to the creation of traps at the oxidised sites (E. M. Speller 2016). It has been shown that 72 hours exposure of the PCBM solution to AM1.5G in air while being stirred oxidises the solution to $3.6 \%$ (H. K. H. Lee et al. 2018). Fullerenes can also form dimers or oligomers (covalently bonded neighbouring molecules of length from at least two bonded molecules) when they bond with each other under exposure to light in an inert atmosphere(Wong et al. 2014) through the formation of carboncarbon bonds(H. K. H. Lee et al. 2018). This oligomerisation can improve the device efficiency and is reversible by thermal annealing as shown in Figure 2.2-3. 


\subsection{Glass transition}

The glass transition temperature is a pseudo-phase transition below the melting point. Above the glass transition, molecules have the mobility to rearrange into a more energetically favourable state such as a crystal. Below the glass transition they are effectively frozen in a glassy, solid, disordered state. The glass transition can be observed in polymers and glassy polymers are typically brittle solids. Discontinuities in thermodynamic properties mark the glass transition temperature.

For some materials, cooling rapidly to below the freezing point does not allow sufficient time for crystals to form, or perhaps the material is an atactic polymer, for example, and it does not crystallise, but there a transition to the glassy state. This transition is marked by a change in the slope of the volume as a function of temperature, indicating a discontinuity in the thermal expansivity. It is distinct from a true phase transition as it is a kinetic property, as opposed to a thermodynamic transition. Further evidence of the transition being kinetic is that the measured transition temperature depends on the rate of cooling (Gao, Koh, and Simon 2013). Experimentally one can perform calorimetry to determine the glass transition temperature, and we measure the point at which there is a discontinuity in the heat capacity as shown in Figure 2.3-1.

There is also a change in slope of the entropy versus temperature, again with a slower rate of cooling leading to a lower measured glass transition temperature. The molecules below the glass transition temperature are not able to sample their possible statistical configurations on the experimental timescale. When extrapolated to absolute zero the glassy system has a finite entropy.

There is a theoretical lower limit to the measurement of the glass transition temperature called the Kauzmann temperature. This is defined as the point at which a lower glass transition temperature, due to a slower cooling rate, intersects with the entropy of a crystalline solid. At this point the lowest energy configuration is the solid form, and the more prolonged timescale of the lowered cooling rate allows time for the molecules to rearrange into a crystal. 


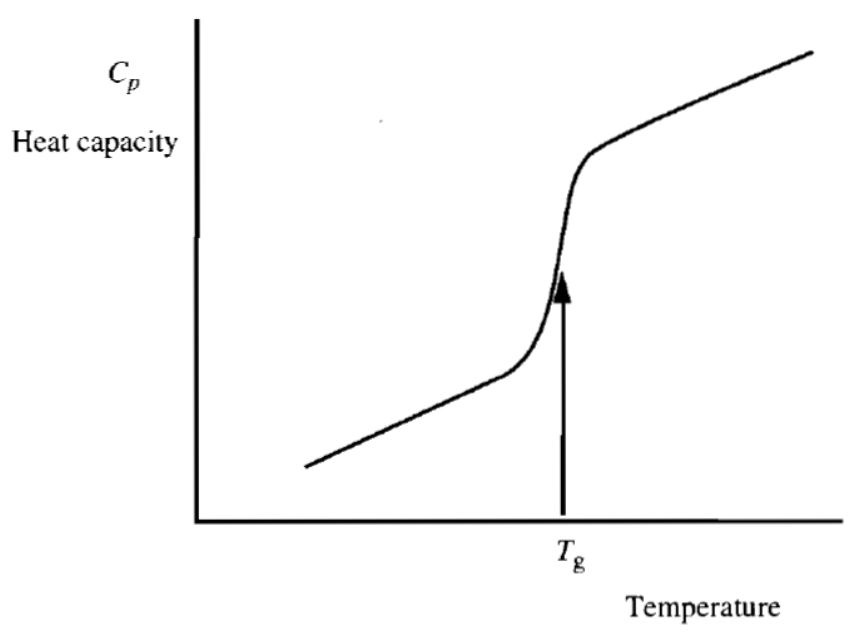

Figure 2.3-1: The discontinuity in heat capacity indicates the glass transition temperature. Reproduced with permission from reference (Jones 2002)

In a binary mixture, the glass transition is a function of the glass transition temperatures of the components, $T_{g i}$, such as in equation (2.11) where $\phi$ is the volume fraction (R. Jones 2002).

$$
\frac{1}{T_{g}}=\frac{\phi}{T_{g 1}}+\frac{1-\phi}{T_{g 2}}
$$

Experiments have shown that for polymers, e.g., polystyrene, the glass transition temperature is depressed by use of lower molecular weight or a thinner film as in Figure 2.3-2 and Figure 2.3-3.

Glass transition temperatures that are lower than the bulk values have been reported for polymer thin-films; for films with one free surface, the effect is observed for thickness $<40 \mathrm{~nm}$. It was shown that it is not purely the thickness of the film, but the presence of a free surface causing enhanced mobility of the polymer. The effect is not apparent in capped films with no free surfaces (Sharp and Forrest 2003). The data shown in Figure 2.3-3 is a compilation over a broad range of studies using different techniques and substrates for PS films with one free surface indicating that these results are reliable and reproducible. 


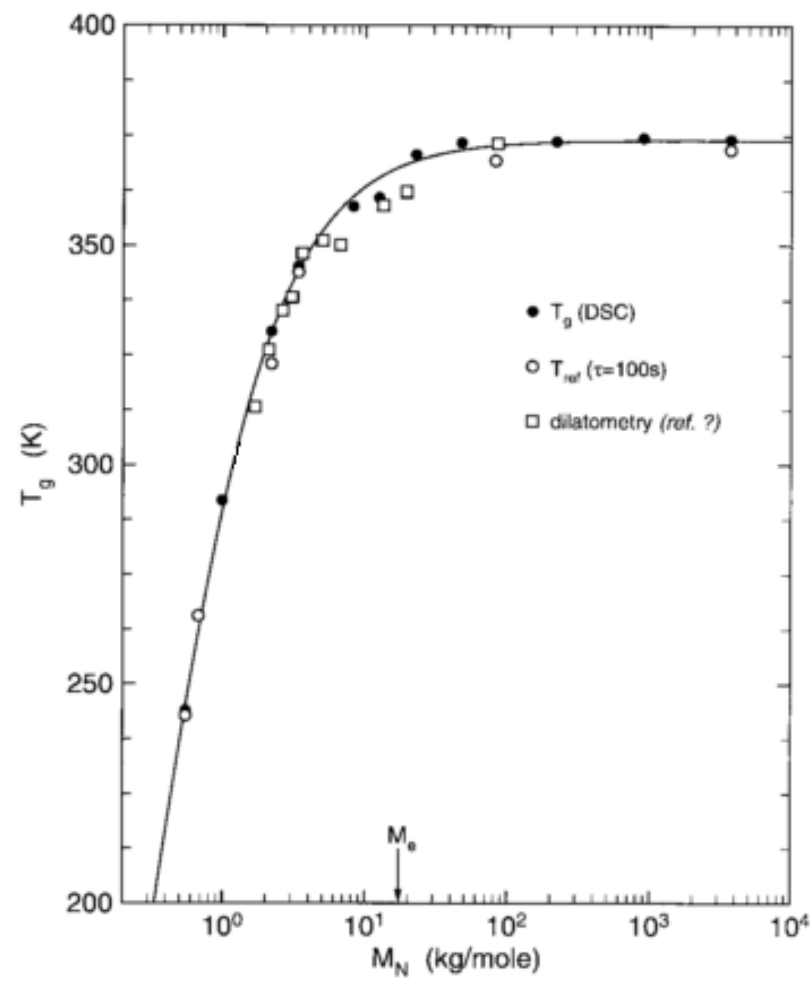

Figure 2.3-2: The glass transition temperature as a function of molecular weight for polystyrene, reproduced with permission from Santangelo and Roland (Santangelo and Roland 1998). $M_{e}$ is the entanglement molecular weight.

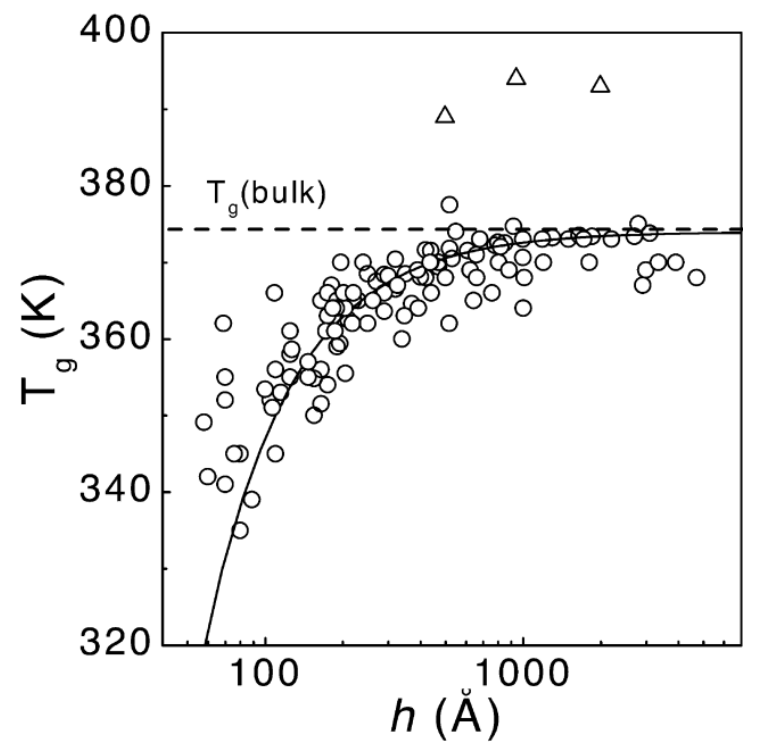

Figure 2.3-3: Glass transition temperature as a function of film thickness $(h)$ for polystyrene. Reproduced with permission from reference (J. A. Forrest and DalnokiVeress 2001).

The enhanced surface mobility at the free surface extends several nanometres into the film and increases the bulk mobility by several orders of magnitude (Ediger and 
Forrest 2014). Keddie et al. were the first to discover this effect and discovered an empirical formula to describe the reduction for PS as

$$
T_{g}(h)=T_{g}^{\text {bulk }}\left[1-\left(\frac{\alpha}{h}\right)^{\delta}\right] .
$$

In this equation, $\mathrm{h}$ is the film thickness and $\alpha$ and $\delta$ are constants with values of 3.2nm and 1.8 respectively (Keddie, Jones, and Cory 1994; J. A. Forrest et al. 1996). Freestanding polymer films with two free surfaces display reductions in $T_{g}$ twice as large than for supported films, suggesting a dependence on the number of free surfaces (Roth and Dutcher 2005).

\subsection{Thermodynamics of binary liquid un-mixing}

To formulate a theory for the miscibility of two substances, it must be possible to calculate the thermodynamic free energy of the mixture (R. A. L. Jones 2002). This will be dealt with in a simple case using the Helmholtz free energy, F, where volume of the system remains constant, defined as

$$
F=U-T S .
$$

$\mathrm{T}$ is the temperature, $\mathrm{U}$ is the internal energy and $\mathrm{S}$ is the entropy of the system. The following theory is a mean-field theory known as the regular solution model. Account is taken of pairwise interactions in a mean-field. A mean field theory is where the field effect of all the particles in a system, on the particle in question, are averaged. The validity of this theory for polymer-polymer systems, and for polymersmall-molecule systems will be examined. Flory-Huggins theory uses a regular lattice to calculate the entropy of organising the sites (which can be taken to be the volume of a monomer, or a small molecule) as in Figure 2.4-1.

The free energy of mixing, $F_{m i x}$, for species $\mathrm{A}$ and $\mathrm{B}$ is

$$
F_{\text {mix }}=F_{A+B}-\left(F_{A}+F_{B}\right) .
$$




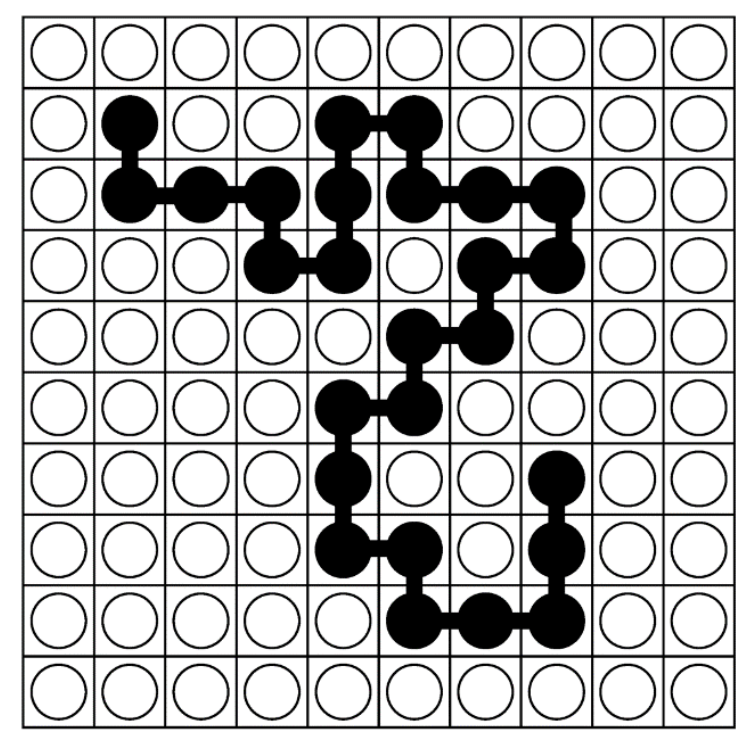

Figure 2.4-1: Lattice describing conformation of a polymer in a solution or mixture with a small molecule. Reproduced with permission from reference (Ender et al. 2012).

To write down the Helmholtz free energy of the mixture, it is necessary to know the internal energy of the mixture, $U_{\text {mix }}$, and the entropy, $S_{\text {mix }}$.

$$
\begin{gathered}
F_{\text {mix }}=U_{\text {mix }}-T S_{\text {mix }}, \\
S_{\text {mix }}=S_{A+B}-\left(S_{A}+S_{B}\right), \\
U_{\text {mix }}=U_{A+B}-\left(U_{A}+U_{B}\right) .
\end{gathered}
$$

The entropy per site of a system, $S$, is given by

$$
S=-k_{B} \sum_{i} p_{i} \ln p_{i}
$$

By assuming that the system is non-compressible, the probability of one site of the system being in a state, $p_{i}$, is the volume fraction of each species, $\phi_{A}, \phi_{B}$, where $\phi_{A}+$ $\phi_{B}=1$.Therefore the entropy of the mixture is

$$
S_{\text {mix }}=-k_{B}\left(\phi_{A} \ln \phi_{A}+\phi_{B} \ln \phi_{B}\right) .
$$

The entropy of mixing for polymers needs to take into account the various possible scenarios(R. Colby; M. Rubinstein; 2003). It can be of similar form to the mixing of a regular solutions if the lengths of the two mixing polymers are equal (the entropy is reduced by a constant factor). Alternatively, if there are two polymers of unequal lengths (with $N_{A}$ and $N_{B}$ monomers in each chain), or there is a polymer in solution or in a mixture with a small molecule (where $N_{A}=N$ and $N_{B}=1$ ), then an asymmetry in the entropy as a function of volume fraction occurs. In all of these cases the 
entropy is modified by taking each monomer of the polymer as a lattice site rather than taking the entire molecule. For a mixture of two polymers

$$
S_{m i x}=-k_{B}\left(\frac{\phi_{A}}{N_{A}} \ln \phi_{A}+\frac{\phi_{B}}{N_{B}} \ln \phi_{B}\right)
$$

In the case of a polymer solution or polymer-small-molecule mixture this gives

$$
S_{\text {mix }}=-k_{B}\left(\frac{\phi_{A}}{N} \ln \phi_{A}+\phi_{B} \ln \phi_{B}\right) .
$$

Because the volume fraction is always less than one, $\phi<1$, the change in entropy will always be positive. The chemical make-up of the blend, i.e. polymers or small molecules, affects the entropy contribution to the free energy: the entropy of mixing for a polymer solution works out to be roughly half of what it would be for a regular solution, taking the same number of lattice sites. More significant entropy changes lead to lower free energies, and, experimentally it is seen that polymers are not as miscible as small molecules. This disposition to phase separate is due to there being fewer possible configurations when the monomers are conjoined into a polymer.

At this point a dimensionless parameter, $\chi$, is defined; called the Flory-Huggins interaction parameter

$$
\chi=\frac{z}{2}\left(\frac{2 u_{A B}-u_{A A}-u_{B B}}{k_{B} T}\right) .
$$

This parameter describes the energetic interactions between the molecules where $u_{i j}$ is the energetic interaction between species $\mathrm{i}$ and $\mathrm{j}$ and $\mathrm{z}$ is the coordination number, or number of neighbours of a particle depending on the dimension of the lattice. This parameter is temperature dependent but is not very sensitive to temperature. Hence the energy of mixing per lattice site is written as

$$
U_{\text {mix }}=\chi \phi(1-\phi) k_{B} T
$$

which holds true for all polymer blends, solutions and regular solutions(R. Colby; M. Rubinstein; 2003).

Substituting these equations into the Helmholtz free energy gives

$$
F_{\text {mix }}=k_{B} T\left(\frac{\phi}{N_{A}} \ln \phi+\frac{1-\phi}{N_{B}} \ln (1-\phi)+\chi \phi(1-\phi)\right) .
$$

The first two terms encourage mixing, although these terms are quite small for long polymer chains. The interaction parameter $\chi$ can be either positive or negative and therefore in polymer mixtures, the value of this parameter controls the miscibility of 
the system. In the case of non-polar molecules where the van der Waals forces dominate the interaction, $\chi$ will be positive; this is the case for the systems in chapter 4. This is for cells on a lattice where it is assumed that each unit takes up the same volume and that the volume does not change on mixing. This is not strictly true in all cases, and a term can be added to account for these differences. The differences arise as some monomers pack better than others when mixed. Complicating factors such as this are usually accounted for by making the $\chi$ parameter a more complex function (e.g. of temperature). The simplest extension of equation (2.22) is to add a constant term so that

$$
\chi=A+\frac{B}{T}
$$

$A$ and $B$ are two constants, and $A$ is referred to as the entropic part while $B / T$ is referred to as the enthalpic part (R. Colby; M. Rubinstein; 2003).

\subsection{Equilibrium and phase diagrams}

The minima of the free energy determine the coexisting compositions. This is because all systems try to lower their free energy in accordance with the laws of thermodynamics. However, these laws also dictate that chemical potentials must be equal at equilibrium, so this will dictate the equilibrium compositions. To understand how the free energy calculation makes predictions about coexisting compositions we will take a graphical approach.

(a)

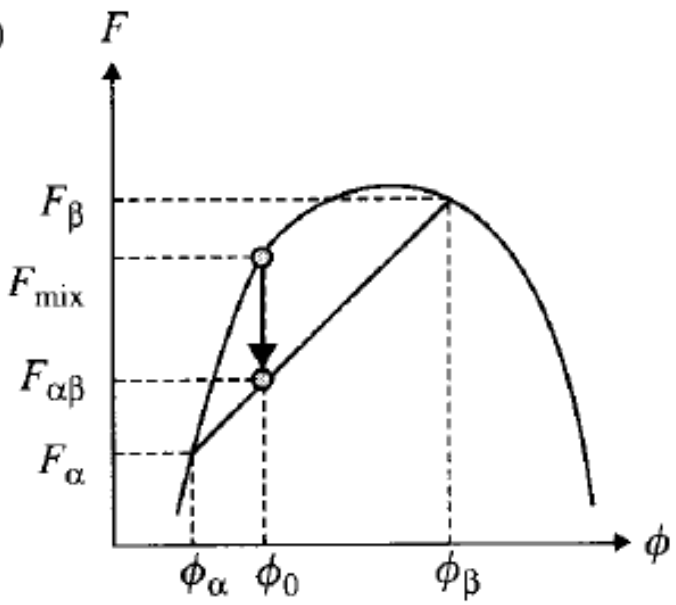

(b)

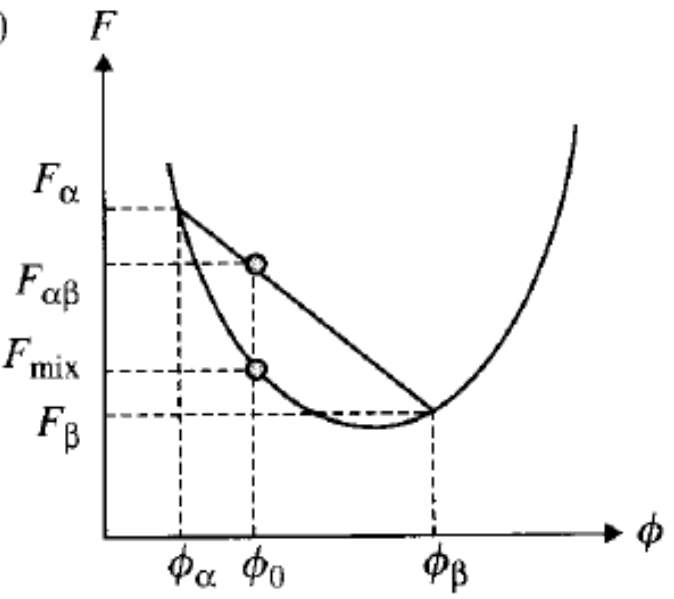

Figure 2.5-1: Free energy curves for (a) unstable and (b) stable configurations. Reproduced with permission from reference (R. Colby, M. Rubinstein, 2003).

For example, we take two simple cases, taking an initial homogenous composition of $\phi_{0}$ with free energy $F_{m i x}\left(\phi_{0}\right)$, it is either locally concave or locally convex, as in 
Figure 6. The determining factor here is whether the free energy of the mixed state is higher or lower than the free energy of a phase separated state, $F_{\alpha \beta}$. The overall composition, $\phi_{0}$, might be made of two phases, with volume fraction of species $\mathrm{A}$ in the $\alpha$ phase, $\phi_{\alpha}$, and in the $\beta$ phase of $\phi_{\beta}$. To find the relative amounts of each phase as a fraction of the total composition we use the lever rule. The fraction of volume, $f_{\alpha}$, has composition $\phi_{\alpha}$, and the fraction of the volume with composition $\phi_{\beta}$ is the fraction $f_{\beta}$. Now the following can be written:

$$
\begin{gathered}
f_{\alpha}=1-f_{\beta}, \\
\phi_{0}=f_{\alpha} \phi_{\alpha}+f_{\beta} \phi_{\beta} .
\end{gathered}
$$

Using these equations to solve for the fraction of each composition it can be found that

$$
\begin{gathered}
f_{\alpha}=\frac{\phi_{\beta}-\phi_{0}}{\phi_{\beta}-\phi_{\alpha}}, \\
f_{\beta}=1-f_{\alpha}=\frac{\phi_{0}-\phi_{\alpha}}{\phi_{\beta}-\phi_{\alpha}} .
\end{gathered}
$$

For simplicity the interfacial energy between the two phases can be neglected and the free energy of the phase separated state, $F_{\alpha \beta}$, can be written as a weighted average of the free energies of the two phases,

$$
F_{\alpha \beta}\left(\phi_{0}\right)=f_{\alpha} F_{\alpha}+f_{\beta} F_{\beta}=\frac{\left(\phi_{\beta}-\phi_{0}\right) F_{\alpha}+\left(\phi_{0}-\phi_{\alpha}\right) F_{\beta}}{\phi_{\beta}-\phi_{\alpha}} .
$$

This linear dependence of the total free energy results in the straight lines we see in Figure 2.5-1 connecting the two free energies of the phase separated compositions and tells their respective volume fractions on the x-axis. If the free energy curve is concave as in Figure 2.5-1 (a) the system can spontaneously lower its free energy by phase separating as $F_{\alpha \beta}<F_{m i x}$.However, as in the case of Figure 2.5-1 (b) where the curve is convex, when $F_{\text {mix }}<F_{\alpha \beta}$, the system is locally stable in the mixed phase. We can write the conditions for stable and unstable states as:

$$
\begin{aligned}
& \frac{\partial^{2} F_{m i x}}{\partial \phi^{2}}<0 \quad \text { unstable } \\
& \frac{\partial^{2} F_{m i x}}{\partial \phi^{2}}>0 \text { stable }
\end{aligned}
$$

In Figure 2.5-2 we have two distinct minima so the free energy will spontaneously lower until it has reached the minimum values joined by a common tangent. This can be explained by the requirement of equilibrium of the chemical potential in the 
system. The chemical potential is the first derivative of the free energy; hence the common tangent indicates the true lowest equilibrium energy compositions of the system.

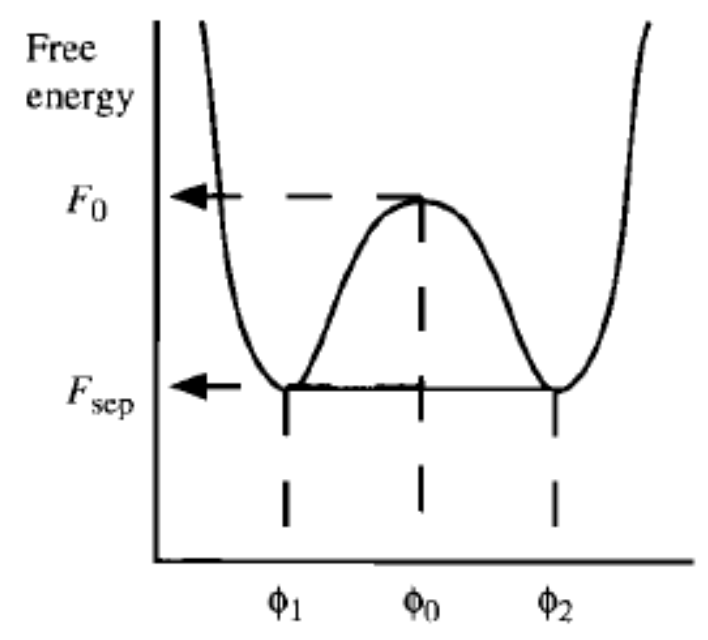

Volume fraction

Figure 2.5-2: A free energy curve with two minima reproduced with permission from reference ( $R$. A. L. Jones 2002)

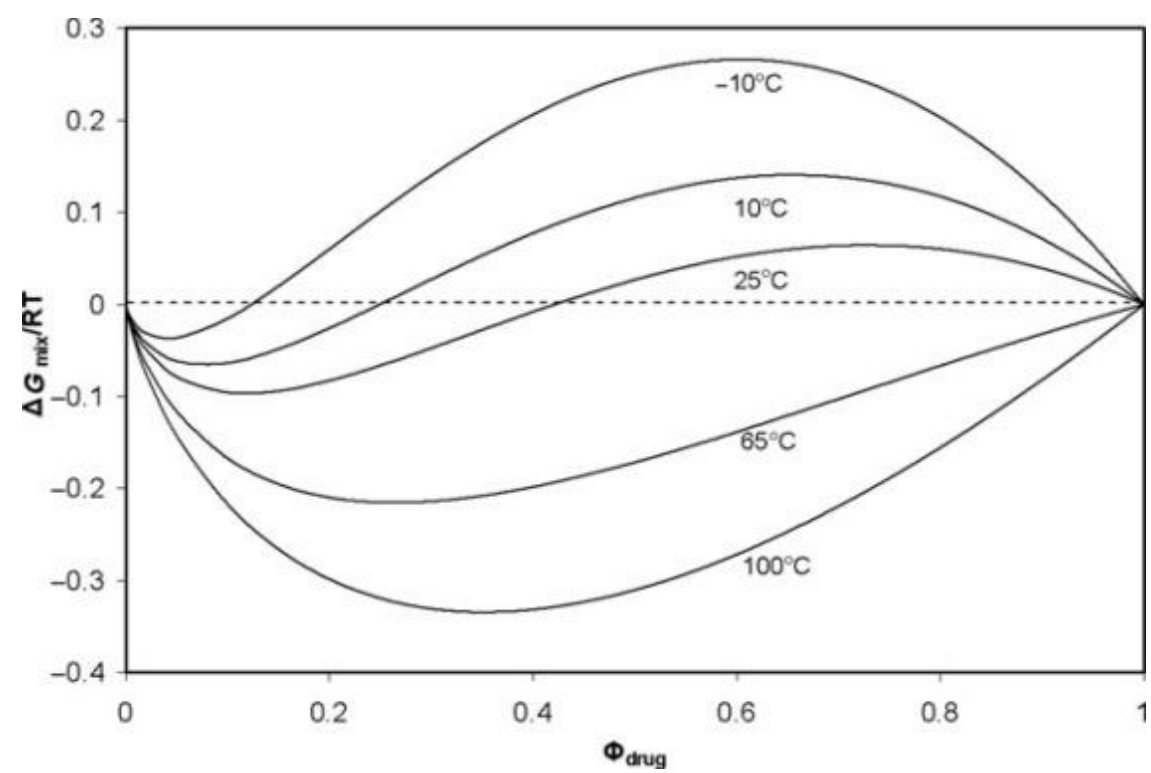

Figure 2.5-3: Example free energy curves (Gibbs free energy of mixing) for different temperatures for $N_{A} \neq N_{B}$ and $\chi=(5 K) / T$. Reproduced with permission from reference ( $Y$. Zhao et al. 2011).

The locus of the coexisting compositions as temperature (and as a result, $\chi$ ) is changed, is known as the coexistence curve or binodal (R. A. L. Jones 2002). A mixture might only be locally stable in respect to small fluctuations. If there exists a lower free energy, then the system is considered metastable. The locus of the points 
of the second derivative is known as the spinodal, which divides stable from metastable regions. It can be noted that there is a critical temperature, $T_{c}$, where the binodal and spinodal lines meet, and this is given by $\frac{d^{3} F}{d \phi^{3}}=0$. As an example, using this regular solution model and a function symmetric around $\phi=1 / 2$, giving the phase diagram shown in Figure 2.5-4. The y-axis can be written as either $\chi$ or $T$.

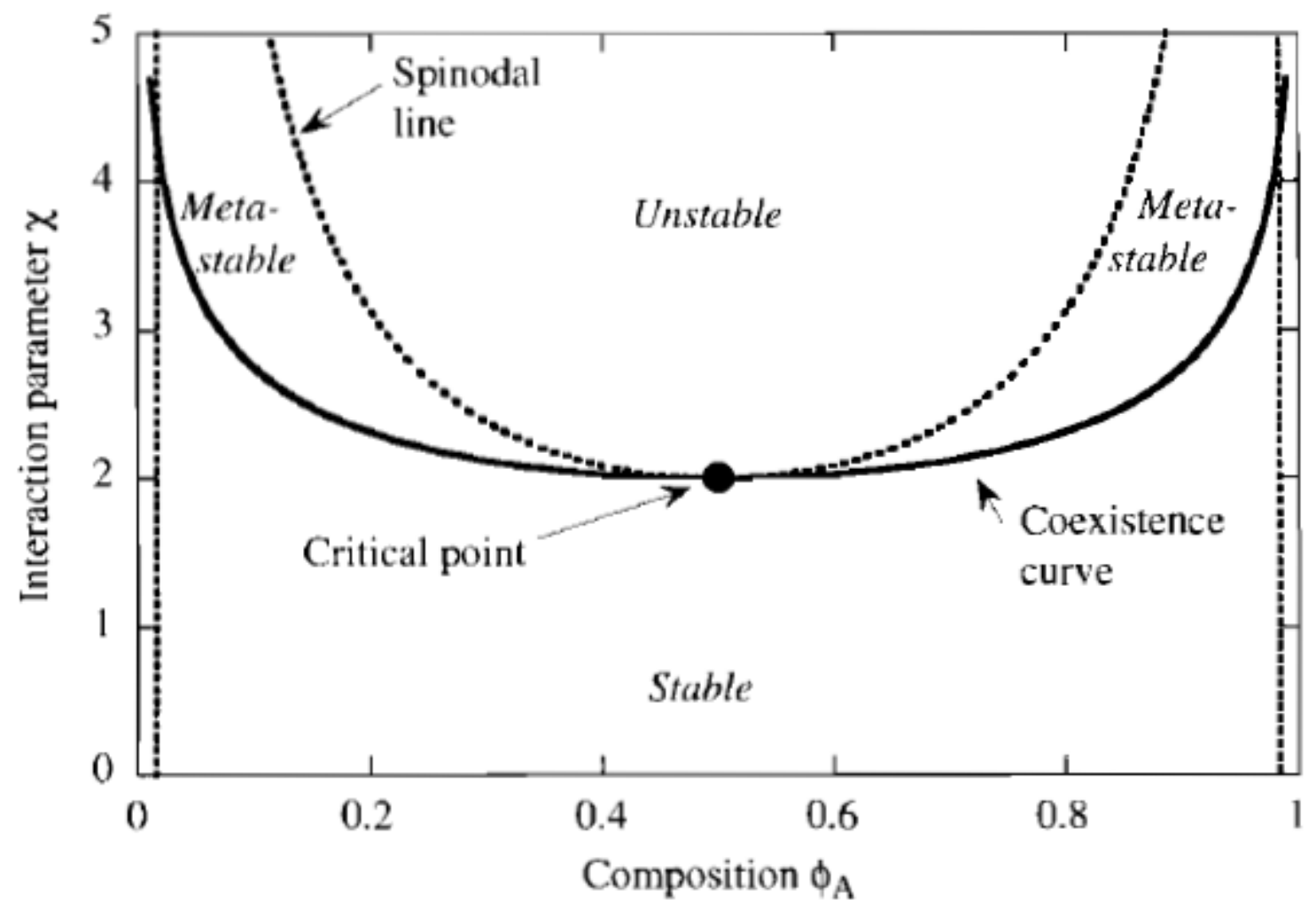

Figure 2.5-4: A phase diagram for a symmetric mixture of small molecules showing the stable, metastable and unstable regions. Reproduced with permission from reference( $R$. A. L. Jones 2002).

The critical interaction parameter, $\chi_{C}$, is given by

$$
\chi_{C}=\frac{2}{N_{\text {ave }}}=\frac{1}{2}\left(\frac{1}{\sqrt{N_{A}}}+\frac{1}{\sqrt{N_{B}}}\right)^{2},
$$

Above this value phase separation occurs. Therefore $N_{\text {ave }}$ is a critical parameter in determining the range of miscibility. $\chi_{c}$ also determines the applicability of mean field theory to finite polymers as will be seen in the following section.

\subsection{Formation of the interface}

The interfacial width, shown schematically in Figure 2.6-1, affects the interfacial tension and the adhesion between two materials. Polymers may, as has been seen, 
mix completely or form domains of different species (coexisting compositions). The phases will have a degree of adhesion that affects the mechanical properties. There is mixing at a molecular level at these interfaces which is a balance of entropic effects; favouring a broader interface, and the energetic penalty of the interaction between the species; favouring a narrow interface. The 'intrinsic interfacial width' is a measure of the distance over which the composition changes from one phase to the other across the interface. To begin, consider an estimation of this width, where a loop of the species B polymer with $N_{\text {loop }}$ units protrudes from the B domain into the A domain, as in Figure 2.6-1, with energy

$$
U_{\text {loop }}=N_{\text {loop }} \chi k T \text {. }
$$

At equilibrium, this energy will be of the order of kT giving

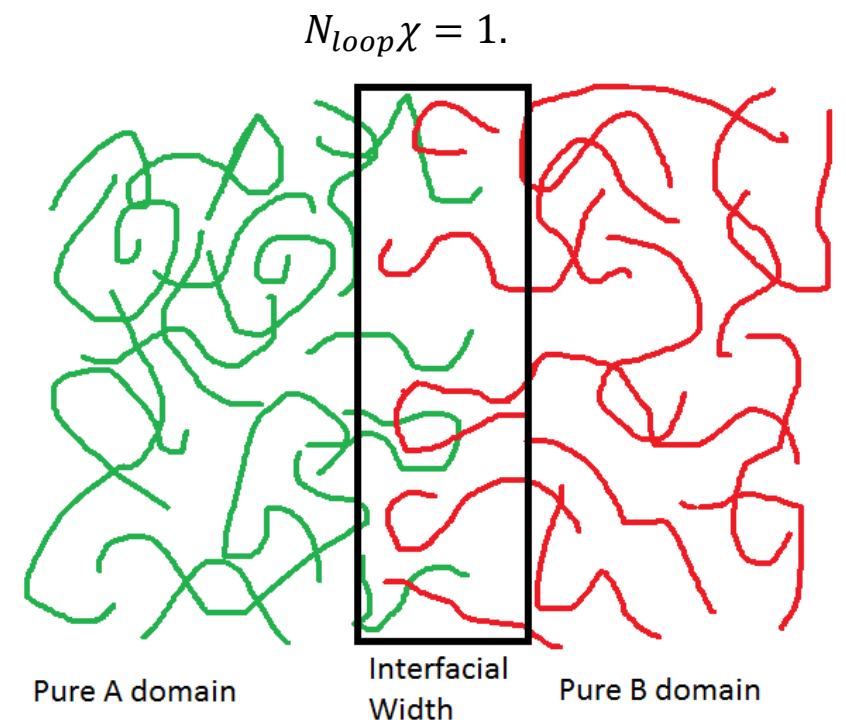

Figure 2.6-1: Schematic of protruding loops illustrating molecular mixing at the interface.

The size of the loop, w, is given by random walk statistics as

$$
w=a \sqrt{N_{l o o p}}
$$

Here $a$ is the statistical step length (R. A. L. Jones 2002). Substitution of equation (2.35) into equation (2.36) gives

$$
w=\frac{a}{\sqrt{\chi}}
$$

Equation (2.37) gives the correct functional form for the interfacial width, which is further refined by square gradient theory and self-consistent field theory (R. A. L. Jones and Richards, R. W. 1999). The addition of a square gradient term to the Flory- 
Huggins free-energy is one method to account for the reduced number of possible configurations of the polymer chains at the interface. Using the square gradient approach, it can be shown that, in the case where $\chi N$ is large, that

$$
w=\frac{a}{2 \sqrt{\chi}}
$$

More accurate results can be found by the self-consistent field theory which at its basis treats the problem of interacting polymers in an analogous way to that of interacting electrons regarding potential energy.

An ideal polymer chain is a random walk with the probability distribution $q\left(\boldsymbol{r}, \boldsymbol{r}^{\prime}, t\right)$. This distribution describes the probability that a polymer chain, $t$ steps long, starts at $\boldsymbol{r}$ and finishes its random walk at $\boldsymbol{r}$ '. In free space, a random walk distribution function obeys a diffusion equation. A diffusion equation can be written, modified by the presence of a spatially varying potential, $\mathrm{U}$ :

$$
\frac{\partial q}{\partial t}=\frac{a^{2}}{6} \nabla^{2} q\left(r, r^{\prime}, t\right)-\frac{U(r)}{k T} q\left(r, r^{\prime}, t\right) .
$$

Equation (2.39) is in the same form as the Schrodinger equation: it describes spatial and temporal behaviour of a particle with probability distribution $\mathrm{q}$ in a potential. Each polymer will experience its own potential $U$, which leads to an intractably large array of equations to solve. To simplify, take a mean-field approach. Assume that each species experiences an average potential with two parts. The first part is a hardcore potential, forbidding two polymers to take up the same space: incompressibility. The second part involves the Flory-Huggins interaction parameter, expressing the chemical attraction between monomers. This attraction is proportional to the average volume fraction for each species. Consider a $\mathrm{z}$-direction interaction for an interface with planar symmetry. The potentials, $U_{A}$ and $U_{B}$, are

$$
\begin{aligned}
& \frac{U_{A}}{k T}=\chi \phi_{B}(z)+v(z), \\
& \frac{U_{B}}{k T}=\chi \phi_{A}(z)+v(z)
\end{aligned}
$$

This is for some function $\mathrm{v}(\mathrm{z})$ which describes the potential and must be chosen so that $\phi_{A}+\phi_{B}=1$.

There is one case where an analytical solution is possible; for the limit of an infinite molecular mass of highly immiscible polymers. In this case, the volume fractions simplify to 


$$
\begin{aligned}
\phi_{A} & =q_{A}{ }^{2} \\
\phi_{B} & =q_{B}{ }^{2}
\end{aligned}
$$

There is no need to integrate along chain lengths as there are no effects of chain ends. The coupled differential equations are:

$$
\begin{aligned}
& \frac{a^{2}}{6} \frac{d^{2} q_{A}}{d z^{2}}=\chi q_{B}^{2} q_{A}+v(z) q_{A}, \\
& \frac{a^{2}}{6} \frac{d^{2} q_{B}}{d z^{2}}=\chi q_{A}^{2} q_{B}+v(z) q_{B} .
\end{aligned}
$$

There is a solution in the limit $\phi_{A}(z \rightarrow-\infty)=0, \phi_{A}(z \rightarrow \infty)=1$ as follows,

$$
\phi_{A}=\frac{1}{2}\left[1+\tanh \left(\frac{z}{w}\right)\right],
$$

where $\mathrm{w}$ is the interfacial width given by

$$
w=\frac{a}{\sqrt{6 \chi}} .
$$

An amendment, with terms inversely proportional to $\chi \mathrm{N}$, for an interface between two polymers of the same $\mathrm{N}$ was found by Tang and Freed (Tang and Freed 1991) and is given by

$$
w(N)=\frac{a}{\sqrt{6 \chi}}\left[\frac{3}{4}\left(1-\frac{2}{\chi N}\right)+\frac{1}{4}\left(1-\frac{2}{\chi N}\right)^{2}\right]^{-1 / 2} .
$$

For two polymers of slightly different lengths to the interfacial width can be described by the following formula derived by Broseta et al. (D Broseta et al. 1990):

$$
w=\frac{2 a}{\sqrt{6\left(\chi-2 \ln 2\left(\frac{1}{N_{1}}+\frac{1}{N_{2}}\right)\right)}} .
$$

In both eqn (2.48) and (2.49), lower $N$ gives a broader interface. This is due to the increased entropy of shorter chains in comparison to longer chains (for a given polymer volume fraction). It has been shown that when $N_{A}=N_{B}$ or $N_{A} \gg N_{B}$, and $N_{B} \gg 1$ mean field theory is applicable (Joanny 1978) however when $N_{B}=1$ the mean field theory is not correct. The validity of mean-field theory is found by evaluating the effects of approaching the critical point, in terms of fluctuations in composition, and the mean field theory is not applicable if the Ginzburg criterion (Binder 1984; Joanny 1978; Wang 2002; Bates et al. 1990) below is met:

$$
\frac{\chi}{\chi_{c}}-1 \leq \frac{1}{N}
$$




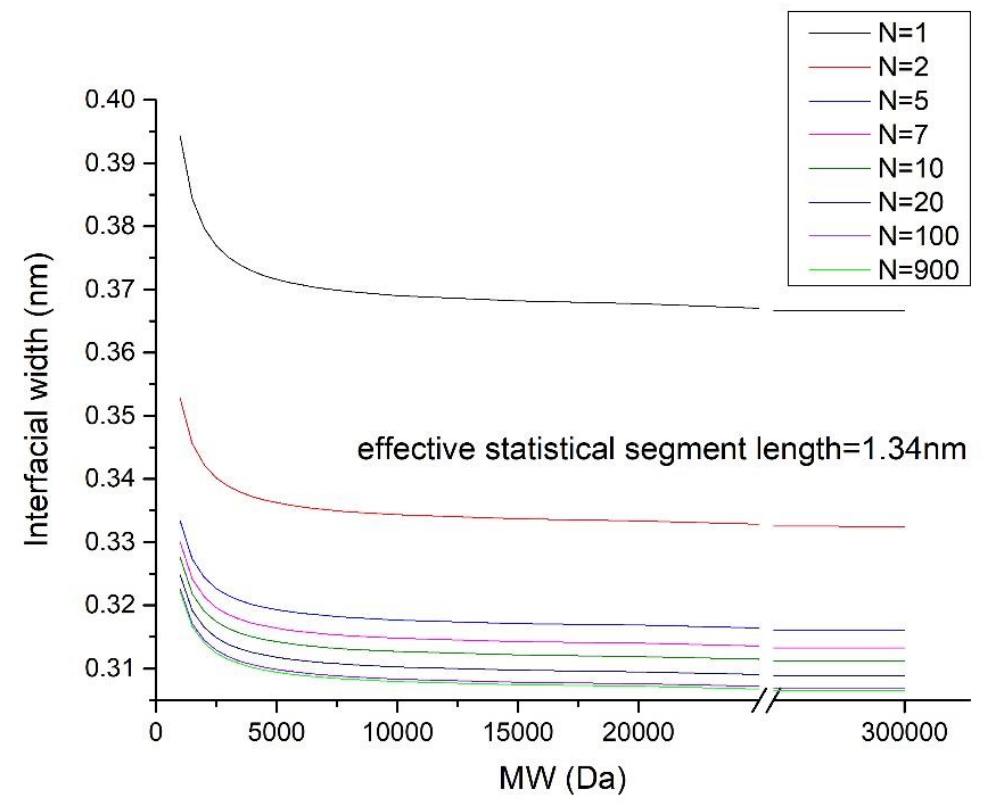

Figure 2.6-2: Plot of equation (2.49) from Broseta et al (Broseta et al. 1990) for various degrees of polymerisation, $N$, on either side of a polymer-polymer interface with $\chi=2$. The MW of 1 monomer is 633Da.

Figure 2.6-2 plots the behaviour of equation (2.49) for the interfacial width. As already stated, this theory is only valid when both molecules have a degree of polymerisation much larger than one. This theory does not apply to our system as $N_{A}=1 \ll N_{B}$. However the plot gives a qualitative idea of the behaviour for polymeric systems; an increase in the interfacial width as either, or both polymer chains become shorter. This graph shows only the prediction for intrinsic interfacial width and excludes any lateral roughness. The $\chi$ parameter value used in Figure 2.6-2 is that calculated for the PS/PCBM system in chapter 4.1, relative to a monomer volume/lattice size equal to that of a PCBM molecule. The statistical segment length is that of PS, also referenced to this lattice size. The values of $\mathrm{N}$ chosen in Figure 2.6-2 cover the range of PS MWs used in chapter 3. Figure 2.6-3 shows the behaviour of equation (2.48) for 2 different $\chi$ parameters and using $N_{\text {ave }}$ from equation (2.35) for $N_{A}=1$ and $N_{B}$ is that of the PS. This shows the same qualitative behaviour with MW as equation (2.49), with the broadening of the interface for lower $\chi$. 


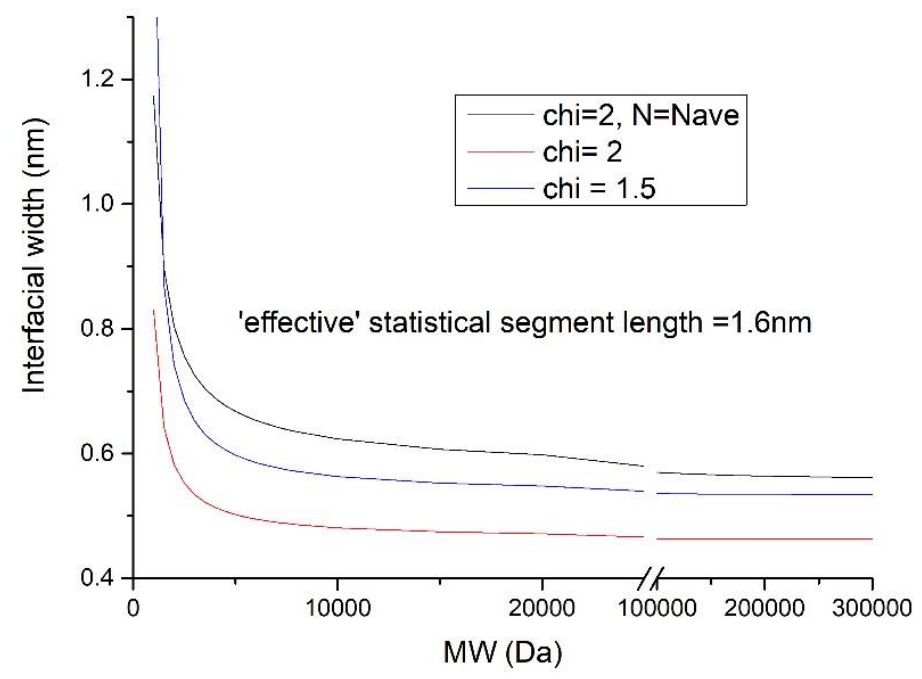

Figure 2.6-3: Polymer-polymer interfacial width from equation (2.48) from Tang and Freed (R. A. L. Jones and R. W. Richards, W. 1999; Tang and Freed 1991) for a symmetric polymer interface (i.e. $\left.N_{A}=N_{B}=N\right)$. The $x$-axis has been converted from number of monomers, $N$, into $M W$ for the full range of PS MWs used in this thesis. The curve is also shown for $N=N_{\text {ave }}$, with $N_{A}$ equal to the number of monomers in a $P S$ chain and $N_{B}=1 . N_{A}, N_{B}$ and $\chi$ are referenced to the volume of a PCBM monomer $\left(\mathrm{V}_{\mathrm{PсBM}}\right)$. The 'effective' statistical segment length, $b_{\text {eff, }}$ is given by $\left(l_{k} l_{\text {ref }}\right)^{0.5}$, where $l_{k}$ is the Kuhn length of PS and $l_{\text {ref }}$ is a reference length equal to $\left(\mathrm{V}_{\mathrm{PCBM}} l_{k}\right) / \mathrm{v}_{\mathrm{k}}$ (with $\mathrm{V}_{\mathrm{k}}$ equal to the volume of a PS Kuhn monomer).This plot for $\chi=2$, corresponds to the experimental situation in section 4.1. The 'effective' statistical segment length is the length required to give the correct prefactor to equation (2.48), given that the $\chi$ parameter in the denominator is referenced to the size of a PCBM molecule (rather than the size of a PS monomer).

\section{Thermal capillary waves}

Figure 2.6-2 and Figure 2.6-3 show predictions for polymer-polymer mixtures for the intrinsic interfacial width. There is another contribution to the measured interfacial roughness, and that is the thermal capillary wave broadening (Sferrazza et al. 1997). The mean squared displacement of the interface due to thermal fluctuations (whose amplitude spectrum in a stable liquid film is cut-off at long wavelengths by either gravitational forces in macroscopic systems, or intermolecular forces, such as van derWaals forces, in thin films) is written $\left\langle\Delta \zeta^{2}\right\rangle$. This displacement adds in Gaussian quadrature with the intrinsic interfacial roughness, $\Delta_{0}$. (NB; Error functions and tanh functions have almost identical shapes when the Gaussian roughness in the error function, $\Delta_{0}$, is equal to the intrinsic interfacial 
width in the tanh function, $w$, multiplied by $\sqrt{2 / \pi}$ ) (R. A. L. Jones and Richards, W. 1999)). ${ }^{1}$ The total roughness is written

$$
\Delta^{2}=\Delta_{0}^{2}+<\Delta \zeta^{2}>
$$

This is related to equations (2.48) and (2.49) by

$$
\Delta_{0}=\frac{\sqrt{2} w}{\sqrt{\pi}}
$$

The capillary broadening is described by

$$
\zeta^{2} \geq \frac{k_{B} T}{4 \pi \sigma_{0}} \ln \frac{\left(\frac{2 \pi}{\Delta_{0}}\right)^{2}}{\left(\frac{2 \pi}{\lambda_{\text {coeh }}}\right)^{2}+\left(\frac{2 \pi}{a_{d i s}}\right)^{2}} .
$$

Here, $\sigma_{0}$ is the interfacial tension, $\lambda_{\text {coeh }}$ is the in-plane coherence length of the neutrons and $a_{d i s}$ is the dispersive capillary length which is given by

$$
a_{d i s}^{2}=\frac{4 \pi \sigma_{0} l^{4}}{A}
$$

where $\mathrm{A}$ is the Hamaker constant and $l$ is the film thickness. Equation (2.53) shows the complex interaction between predicted interfacial width and the interfacial roughness measured by NR. The measured roughness is, in principle, dependent on the thickness of the film.

\subsection{Thermodynamics of the liquid-solid transition}

In the liquid-solid transition, the state of the order changes discontinuously, making it a first order phase transition. There is a change in entropy, $\Delta S_{m}$, related to the latent heat that is released, $\Delta H_{m}$, and the melting temperature, $T_{m}$ :

$$
\Delta S_{m}=\frac{\Delta H_{m}}{T_{m}} .
$$

If a liquid is held at exactly its melting temperature, however, it will not crystallise. This is because the formation of a crystal creates interfacial energy, $\gamma_{s l}$, between the liquid and the solid. $T_{m}$ is defined as the temperature at which the free energies of the liquid and the solid are equal. Because of the energetic penalty from the interface it is necessary to undercool a liquid to achieve crystallisation. It is also necessary to give the system time to sample the microstates of the least energetic configuration, below this temperature, the resulting crystalline phase will be in a more highly

\footnotetext{
${ }^{1}$ Throughout the thesis, the term roughness refers to characterisation using an error $\mathrm{fn}$ which is a convolution of a Gaussian with a step function; and the word width refers to characterisation using a tanh fn (also referred to as intrinsic width).
} 
ordered state than the liquid. Crystallisation usually occurs via a nucleation and growth mechanism, it might take some time for a sufficiently large initial crystalite to form that overcomes the penalty for the crystal/melt interface.

There will be a change in the free energy, $\Delta G(r)$, upon nucleation of a spherical crystal of radius $r$ of

$$
\Delta G(r)=\frac{-4}{3} \pi r^{3} \frac{\Delta H_{m}}{T_{m}} \Delta T+4 \pi r^{2} \gamma_{s l}
$$

This is shown graphically in Figure 2.7-1 (Perrin, Musa, and Steed 2013) where it is clear there is a maximum in the total change in Gibbs free energy at a critical crystal radius, $r^{*}$, given by:

$$
r^{*}=\frac{2 \gamma_{s l} T_{m}}{\Delta H_{m} \Delta T}
$$

This critical radius determines the stability of a crystal, if the crystal is larger than $\mathrm{r}^{*}$ it will continue to grow as it will lower its free energy, but if it is smaller it will remelt (R. A. L. Jones 2002).

The free energy barrier, $\Delta G^{*}$, is

$$
\Delta G^{*}=\frac{16 \pi}{3} \gamma_{s l}^{3}\left(\frac{T_{m}}{\Delta H_{m}}\right)^{2} \frac{1}{\Delta T^{2}} .
$$

This temperature dependence results in significant nucleation only being typically

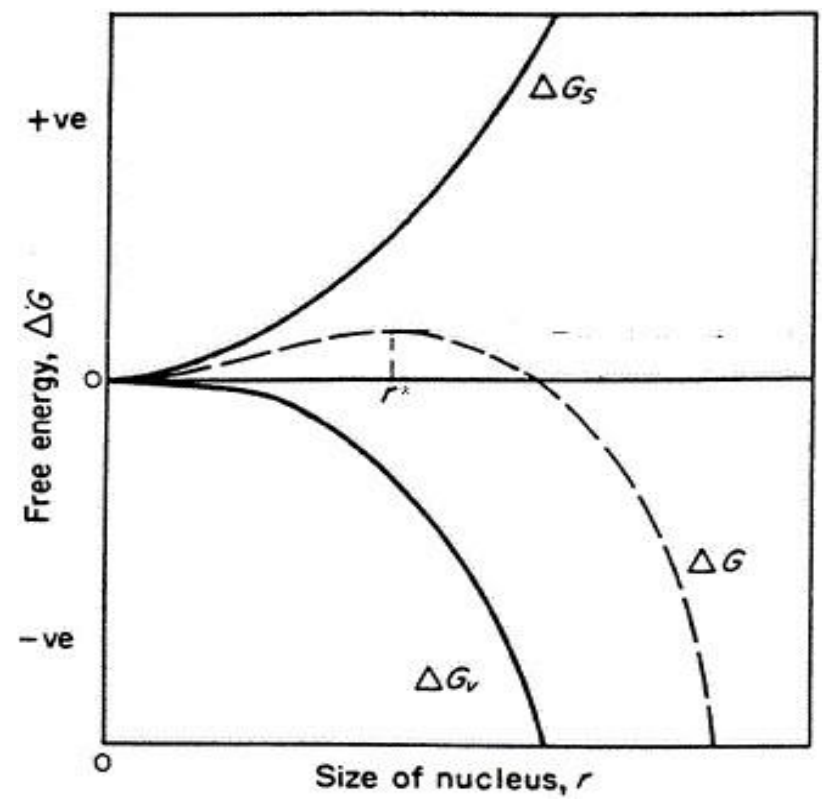

Figure 2.7-1: $\Delta G_{v}$ is the energy change in the bulk and $\Delta G_{s}$ is the energy change of the surface. $\Delta G$ is the total gibbs free energy change. Reproduced with permission from reference (Perrin, Musa, and Steed 2013). 
experimentally observed when the system is tens of degrees below $T_{m}$ (R. A. L. Jones 2002). This theory is called homogeneous nucleation and is true only for a pure liquid free of defects or foreign particles. A much more common type of nucleation in physical systems is heterogeneous nucleation which accounts for the presence of defects or walls of the container to nucleate crystal growth. The defects lower the activation energy substantially, and in practice, we see extensive crystallisation only a few degrees below $T_{m}$ (R. A. L. Jones 2002).

The increase in free energy on melting is greater further from $T_{m}$; which leads to an increase in nucleation as $\mathrm{T}$ is lowered. There is also an opposing factor that the mobility of the molecules is lowered as T is lowered and so nucleation and growth are slower. Thus there is a maximum region of temperature between the melting and glass transition temperatures where nucleation is at its greatest, which has been shown for $\mathrm{PCBM}$ to be at $\mathrm{T}=150^{\circ}-170^{\circ} \mathrm{C}$ (Lindqvist et al. 2013).

The Avrami equation describes the growth of a crystal front into volume $V_{c}$ with an Avrami index, $\mathrm{n}$, a crystallisation rate constant, $\mathrm{k}$, over a time $\mathrm{t}$ (Lorenzo et al. 2007) by

$$
1-V_{c}(t)=e^{-k t^{n}}
$$

Crystal growth in PCBM can take many forms; the fullerene can pack in different ways each representing a local energy minimum and with each structure having different electronic properties (Volonakis, Tsetseris, and Logothetidis 2012). PCBM has been shown to form both nanometre-sized crystals(Môn et al. 2015; Hopkinson et al. 2011; Verploegen et al. 2010) and needle-like micron-sized crystals (Swinnen et al. 2006), as in Figure 2.7-2. It is evident in these images that there is some branching of the needles when cast from a 1:2 solution and annealed at $125^{\circ} \mathrm{C}$. This data is from a blend of P3HT:PCBM. 


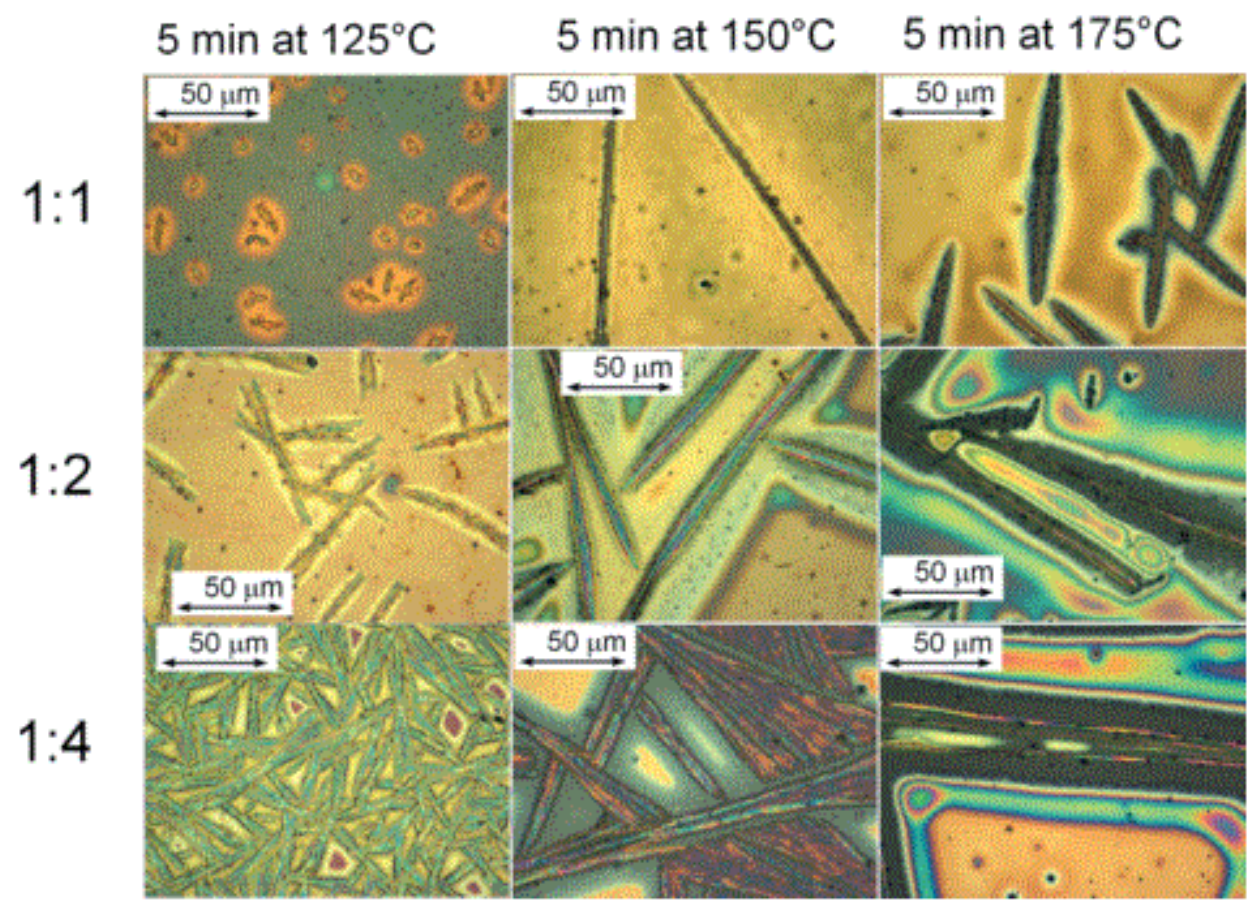

Figure 2.7-2: This shows various crystal sizes and branching for different blend rations (indicated on the vertical axis) for different annealing temperatures (indicated on the horizontal axis). Reproduced with permission from reference (Swinnen et al. 2006).

Such branching may be an early indication of the formation of a spherulite. A spherulite is a roughly spherical (or circular in a confined 2D geometry) formation of a crystal, usually produced by nucleation fronts growing in all directions from the nucleation site, but they can also be formed from needle-like crystals where there is a secondary growth front nucleated along the crystal length as shown by Figure 2.7-3.

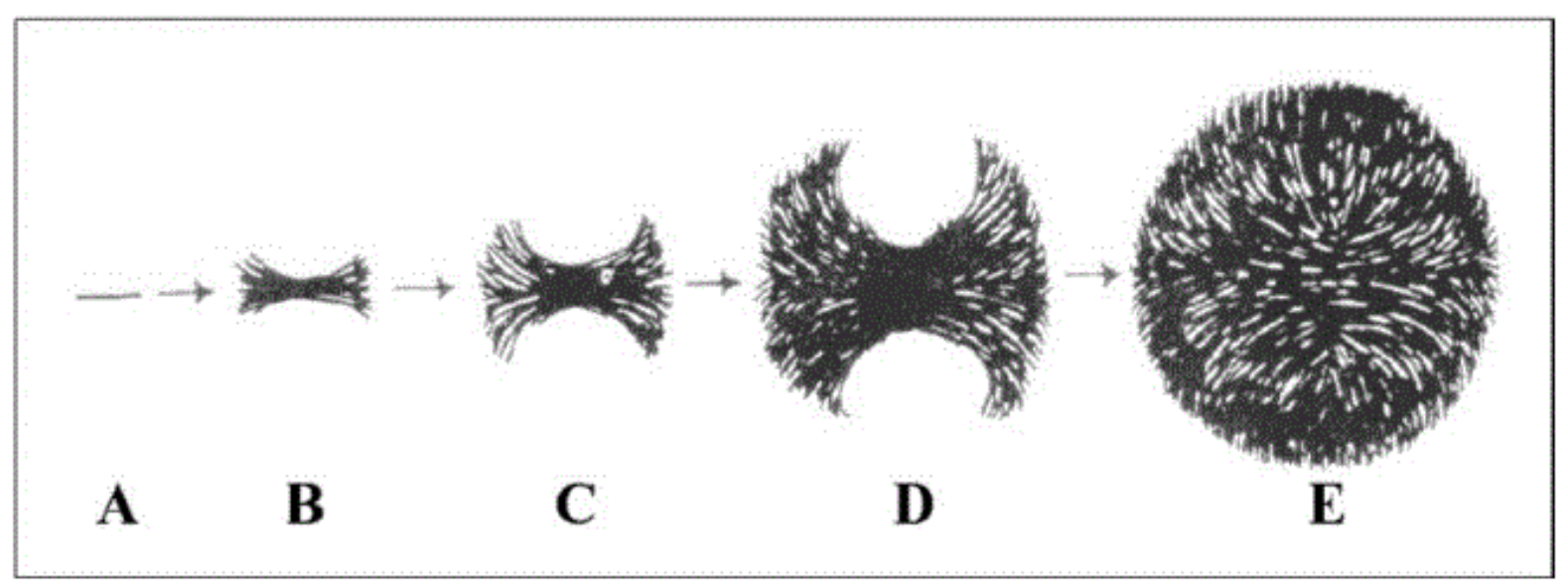

Figure 2.7-3: The progression from a single needle (A) to a branched needle (B) to a spherulite(E). Reproduced with permission from reference (Granasy et al. 2005). 
This occurs when the reorientation of the crystallising molecules is much slower than the interface propagation leading to difficulty aligning with the parent crystal (Granasy et al. 2005)

\subsection{Summary}

Predictions for interfacial width from SCFT, and mixing from Flory-Huggins theory will be compared to experimental results for two model fullerene/PS bilayer systems. Flory-Huggins theory for polymer/small-molecule systems will be compared quantitatively with measurements of coexisting compositions in thin-film bilayers. The predictions for interfacial width as a function of MW will be compared qualitatively to the existing theory. The available theory for interfacial width was derived for polymer/polymer systems and, as such, cannot be directly compared to polymer/small-molecule systems due to the possible effects of composition fluctuations. The effects of light-induced oxidation and oligomerisation of the PCBM, on the mixing behaviour of bilayer systems, will be presented and compared with the findings for the non-illuminated PCBM/PS bilayers. The observations of two micron-sized PCBM crystal morphologies (needle-like shapes and fan-like shapes) will be presented. These morphologies have been observed in previous work and will be examined as a function of annealing temperature, MW of the polymer and thicknesses of the layers in the film. GIXD is used to monitor any (nano) PCBM crystal growth that might occur within the bilayers. 


\section{Materials and Methods}

This section contains details of the materials and experimental methods used in this thesis. The materials were chosen to allow for a detailed study of the effect of MW on the composition profiles of polymer/fullerene systems. PS was chosen as it is available with low polydispersity and PCBM was chosen as it is the benchmark fullerene used in OPV devices. The methods detailed below were chosen to compliment our bilayer geometry: NR allows for measuring a depth profile normal to the substrate. Other methods were used to check the quality of the samples following annealing. This was to check for crystallisation or degradation of the materials which would adversely affect our results.

\subsection{Materials}

\subsubsection{Fullerenes}

The fullerenes used were PCBM and bis-PCBM with a purity of 99.5\% which was purchased from Solenne. The non-fullerene acceptor (NFA) used was a difluorobenzothiadiazole -based polymer (O-IDFBR). This is a small molecule and was synthesised at Imperial College London (Holliday et al. 2016).

\subsubsection{Polymers}

Two polymers were used, atactic polystyrene, PS, and poly[N- 9'-heptadecanyl-2,7carbazole-alt-5,5-(4,7-di-2-thienyl-2',1',3'-benzothiadiazole], PCDTBT. Six different PS molecular weights were used. The nominal molecular weights were 2000, 3500, 5000, 20000, 100000, 200000 and 300000. The weight average molecular weights for these were 1860, 2930, 4730, 18,500, 111,400, 224,200 and 278,200 Da respectively. The polydispersity of these polymers was 1.04, 1.04, 1.04, $1.03,1.03,1.03$, and 1.05 respectively. PCDTBT was obtained from Ossila with a molecular weight of 68,000Da. PS was obtained from Agilent Technologies.

\subsubsection{Substrates}

Two silicon substrates (with a native oxide layer) were used. Four inch diameter wafers of thickness 525 microns, from Prolog Semicor (Ukraine). These were cleaved into smaller pieces $(\sim 1 \mathrm{cmx} 1 \mathrm{~cm})$ prior to making bilayer samples. Thicker 
substrates were used for the neutron scattering experiments. These wafers were 2 inch diameter and of thickness 1150 microns from Siltronix, France. The orientation of both was 100 . Mica sheets of size $65 \mathrm{~mm} \times 65 \mathrm{~mm}$ and thickness $0.15 \mathrm{~mm}$ were purchased from Goodfellow, Cambridge.

\subsubsection{Solvents}

Toluene was used purchased from Sigma Aldrich of purity 99.9\%. Chlorobenzene was used and purchased from Sigma Aldrich of purity 99.9\%. Isopropanol (IPA) was purchased from Fluka Analytical of purity 99.9\%. Acetone was purchased from Sigma Aldrich of purity $99.9 \%$

\subsubsection{Water}

De-ionised water was obtained from an Elix Millipore system.

\subsection{Sample Preparation}

In this study, single layer or bilayers were prepared on top of silicon substrates. The silicon substrates were sonicated for fifteen minutes in acetone and then isopropanol, also for fifteen minutes. They were then rinsed with deionised water and dried by spinning on the spin coater to remove any remaining solvent. We deposited the bottom layer directly onto the silicon substrate by spin coating. The top layer was spin coated onto a mica substrate which had been freshly cleaved and then floated on top of the first layer from a bath of de-ionised water. This was done by attaching one edge of the mica sheet to a clamp so that it was hanging vertically. The silicon substrate (with a PCBM layer) was placed flat below the surface of the water. Then the mica was slowly lowered into the water such that the PS layer remains attached to the mica at one edge and floats above the silicon substrate. Then the water level was lowered by use of a siphon until the PS was deposited onto the substrate, forming the top layer of the bilayer. This created two distinct layers later confirmed by neutron reflectivity. The samples were then dried under vacuum for at least 2 hours. Following this drying, the samples were annealed under vacuum or under dry nitrogen for varying times and temperatures. When annealed under nitrogen a Linkam Controller THMS600 heating stage was used with a liquid nitrogen pump LNP95. 
To determine the film thicknesses, single layer samples of both polymer and fullerene were prepared on silicon substrates and scratched. The depth of the scratch was then measured using AFM which allowed us to determine which concentrations and spinning speeds gave the desired thicknesses; for the majority of samples this was 40-60nm for PS and 20nm for PCBM.

\subsubsection{Solutions}

Various solutions of different concentrations were used to spin coat depending on the desired film thickness. PS was dissolved in toluene and PCBM in chlorobenzene. PCDTBT was dissolved in chlorobenzene and spin-coated onto mica. The solutions were measured by percentage weight using a microbalance (Sartorius). The balance measured in five figures in grammes to 4 decimal places

In the interests of avoiding contamination, the tweezers were rinsed with the solvent before use and pipettes were disposed of after each use. The deionised water in the floating bath was also changed between floatings. Further, fresh vials and lids were used for each solution

\subsection{AFM}

Atomic force microscopy (AFM) is a tool developed from scanning tunnelling microscopy (STM) for measuring surface profiles of both conductive and insulative materials with atomic resolution (when used under ultra-high vacuum). The AFM is based on the measurement of forces by deflection of a cantilever, treated as a spring when an atomically sharp tip interacts with the surface forces of a sample.

To make AFM sufficiently sensitive, a soft spring is needed to enable deflection of the tip for a change in the height of the sample surface. To minimise vibrational noise a stiff spring with a high resonant frequency is desired. The resonant frequency of the spring, $f$, is given by

$$
f=\frac{1}{2 \pi}\left(\frac{k}{m_{0}}\right)^{1 / 2}
$$

$m_{0}$ is the effective mass and $\mathrm{k}$ is the spring constant. It is necessary to decrease both of these values to obtain a soft spring with a high resonant frequency (Binnig and Quate 1986). 


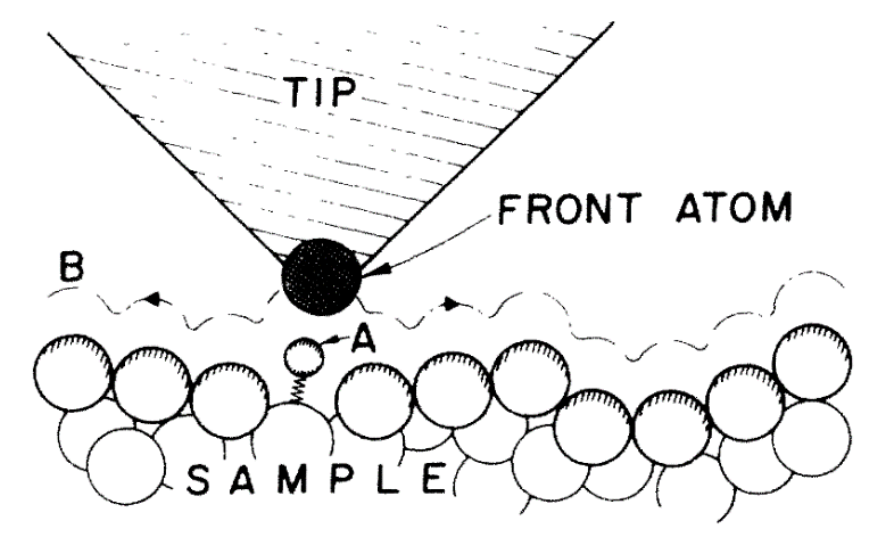

Figure 3.3-1: The tip responds to the force felt by the atoms on the surface and generates the trace, B. Reproduced with permission from reference (Binnig and Quate 1986).

The potential energy of the tip-sample interaction is given by $V_{t s}$ and the force is given by $F_{t s}=-\partial V_{t s} / \partial z$. The spring constant is given by $k_{t s}=\frac{-\partial F_{t s}}{\partial z}$. The force is used as the imaging signal. The force is comprised of short-range chemical forces and long-range van der Waals, electrostatic and magnetic forces. In air, there are also meniscus forces of adhesion between the sample and the tip.

A beam of light measures the deflection of the tip as it moves across the sample surface: a laser is shone on the tip and the reflected signal read by a photodiode as in Figure 3.3-2. Sample surface topography is measured using a feedback loop in which the probe is moved vertically so as to maintain a constant signal on the position sensitive detector (split photodiode). There are three possible modes of operation of an AFM: static or contact mode, non-contact mode and dynamic or tapping mode. In static mode the tip moves across the surface either keeping the force of contact constant and measuring the height or by keeping the height above the sample constant and measuring the force, the tip is less than a few angstroms above the sample surface and the force felt is repulsive. In dynamic mode there are two situations: amplitude modulated and frequency modulated, in both of which the tip is vibrated to tap at the surface from a height of several nanometres to $200 \mathrm{~nm}$. This mode reduces the adhesion effects so is typically used for biological samples or in ambient conditions. Amplitude modulation involves a fixed frequency and changes in the amplitude and phase are used to measure the force (or the amplitude and hence 
force are kept constant when measuring surface topography). The frequency of the tip is just above the resonant frequency. Frequency modulated AFM involves resonating the tip at its resonant frequency. In non-contact mode, the tip is at its resonant frequency and interactions with the surface change this resonant frequency due to van der Waals forces primarily, which are attractive as the tip is held tens to hundreds of angstroms above the surface. Figure 3.3-3 shows the form of the van der Waals force which dominates in AFM measurements particularly of larger sample features (larger than atomic resolution). In this thesis, tapping mode was used for all measurements.

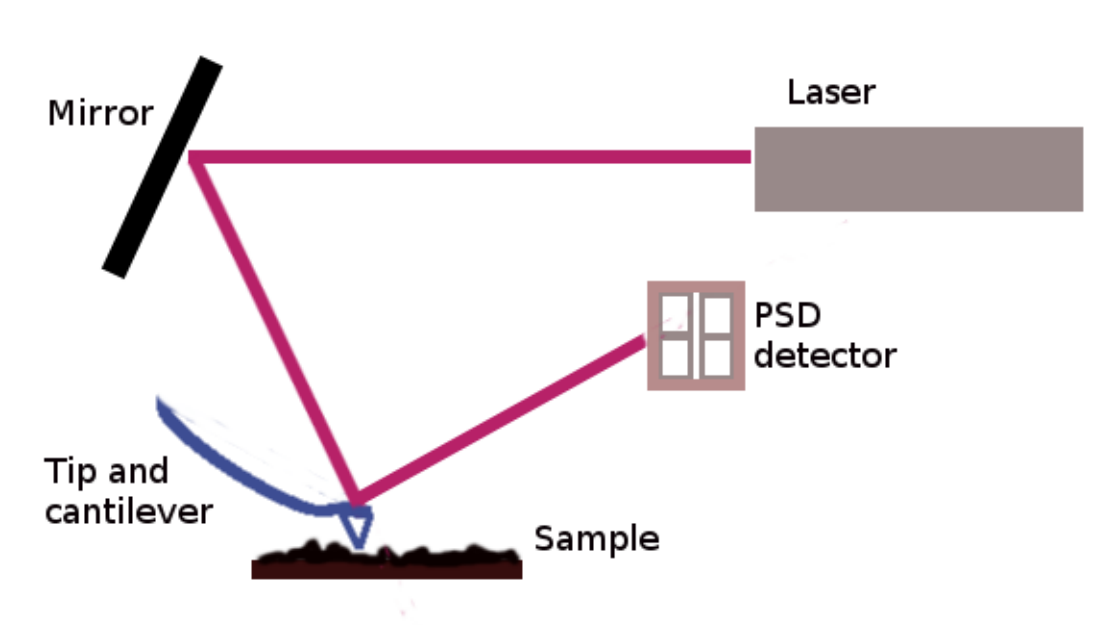

Figure 3.3-2: Schematic diagram of an AFM sowing the laser light hitting the tip before being reflected into the position sensitive detector (PSD).

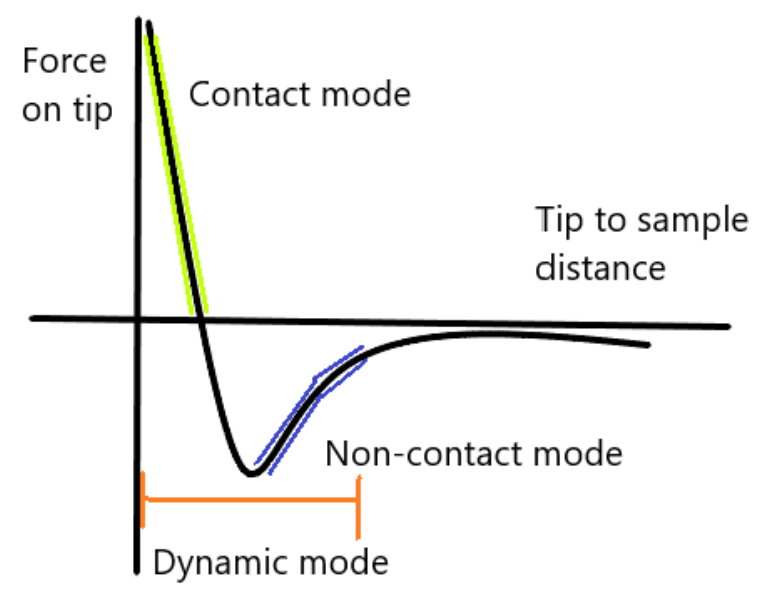

Figure 3.3-3: Diagram of forces felt by the tip as it approaches the sample and which region applies to each scanning mode. 


\subsection{Optical Microscopy}

The basic premise of optical microscopy $(\mathrm{OM})$ is the refraction of light through a lens making the object in question appear larger. The Gaussian lens formula describes the relationship between the focal length, $\mathrm{f}$, the distance from the object to the lens, $S_{1}$, and the distance from the lens to the image, $S_{2}$.

$$
\frac{1}{S_{1}}+\frac{1}{S_{2}}=\frac{1}{f}
$$

Microscopic resolution is quantified by the numerical aperture (NA) which is defined as in equation (3.3), where $\mathrm{n}$ is the refractive index of the medium between the lens and the specimen (usually air) and $\alpha$ is half the angle of the cone of light received by the lens.

$$
N A=n \sin \alpha
$$

Using this definition the minimally resolvable distance can be calculated, $d$, in $\mu \mathrm{m}$, as in equation (3.4) where $\lambda$ is the wavelength of the light being used to illuminate the sample.

$$
d=0.61 \lambda / N A
$$

Another relation using NA is the depth of resolution, Z, which determines the depth on an imperfect sample surface which will be in focus.

$$
Z=\frac{n \lambda}{N A^{2}}
$$

It is clear from equation (3.5) that the larger NA, the shallower the depth of resolution will be. Polarised light can be used to ascertain crystal planes in optically anisotropic features such as spherulites. In the case of spherulites, a maltese cross is formed whose orientation lines up with the direction of the polariser. Light can be vertically or horizontally polarised whereby the light from the light source is passed through a polariser which prevents all oscillatory components from passing through bar those in the direction of the polariser (Murphy and Davidson 2013). The microscopes used were a Zeiss AX10 for the colour images and a Nikon Eclipse E600FN for the greyscale images. 


\subsection{ATR-FTIR}

Fourier transform infrared spectroscopy (FTIR) is a useful tool for analysing the chemical constituents of solid or liquid samples. It is a vibrational technique, that exploits the fact that stretching and bending of interatomic bonds and groups of bonds within molecules, gives rise to well-defined absorption peaks in the infrared. Attenuated total reflection (ATR) is a variant of FTIR that allows for the investigation of films and surfaces. It involves using a prism of high refractive index (at least higher than that of the sample) which totally internally reflects a beam of light. The internal reflection produces an evanescent wave that can permeate a sample which is in contact with the prism surface. The necessary condition for obtaining a clear spectrum is to apply a significant force to the sample material so that it has good contact with the prism. The intensity of the evanescent wave propagating through the sample decays exponentially with distance, usually on the order of microns.

If the sample is absorbing, then some of the evanescent wave will be absorbed. The reflectance is thus attenuated by the selective wavelength absorbance of the sample, and this produces characteristic dips in the reflectance (Melosivic 2012).

To produce total reflection the critical angle, $\theta_{c}$ (given by equation (3.6)), must be exceeded.

$$
\theta_{c}=\sin ^{-1} \frac{n_{2}}{n_{1}}
$$

where, $n_{2}$ is the refractive index of the sample and $n_{1}$ is the refractive index of the ATR crystal (Spectra-Tech 1990). FTIR-ATR was used to assess the effectiveness of the annealing procedures used in the thesis, in terms of preventing any chemical degradation of the PS or PCBM. Of the three different annealing procedures used in the thesis, the ex-situ annealing of the NR samples is the procedure worthy of closest examination. This is because of the need here to briefly expose the samples to air at elevated temperatures at the end of annealing (the vacuum oven takes around 2 minutes to fill with air and enable the door to be opened). This procedure has been used previously (with the same model of vacuum oven) on conjugated polymer systems, and did not show any signs of material degradation (up to the maximum oven set point of $200^{\circ} \mathrm{C}$ ) (A. M. Higgins et al. 2006). To check that no degradation occurred in the present study, PS $\left(\mathrm{M}_{\mathrm{w}}=1860\right)$ and PCBM single layers were 
deposited onto silicon substrates by drop casting and annealed using the same protocol as used for the ex-situ NR samples. The annealing temperatures and times used in these tests were $170{ }^{\circ} \mathrm{C}$ or $180{ }^{\circ} \mathrm{C}$ for 30 minutes (higher temperatures and longer times than used for any of the ex-situ NR samples). FTIR was performed using a Perkin Elmer Frontier spectrometer, with a diamond ATR crystal. The FTIR measurements on PS were performed by taking a background and then pressing the PS film (still attached to the silicon substrate) onto the ATR crystal. The PS film was sufficiently thick to ensure that no signal from the silicon substrate was observed. The PCBM films were thinner than the PS films, and so had to be removed from the silicon substrate to avoid the PCBM absorption peaks from being dominated by that from the silicon. The PCBM films were scraped from the silicon using a scalpel and pressed onto the ATR crystal using a potassium bromide crystal (which has a high and constant transmission across the part of the infrared spectrum of relevance to degradation by exposure to air). Representative spectra from PS films and PCBM films before and after annealing are shown in Figure 3.5-1. The key comparison for PS relates to the growth in the intensity of several peaks at wavenumbers 1650-1800 $\mathrm{cm}^{-1}$ and $3100-3600 \mathrm{~cm}^{-1}$ which is known to occur following oxidation (Mailhot and Gardette 1992), and for PCBM relates to the emergence of a peak at $1782 \mathrm{~cm}^{-1}$ in addition to that at $1737 \mathrm{~cm}^{-1}$ (Emily M. Speller et al. 2017). No evidence for oxidation of the PS or PCBM is seen in any of the spectra. 

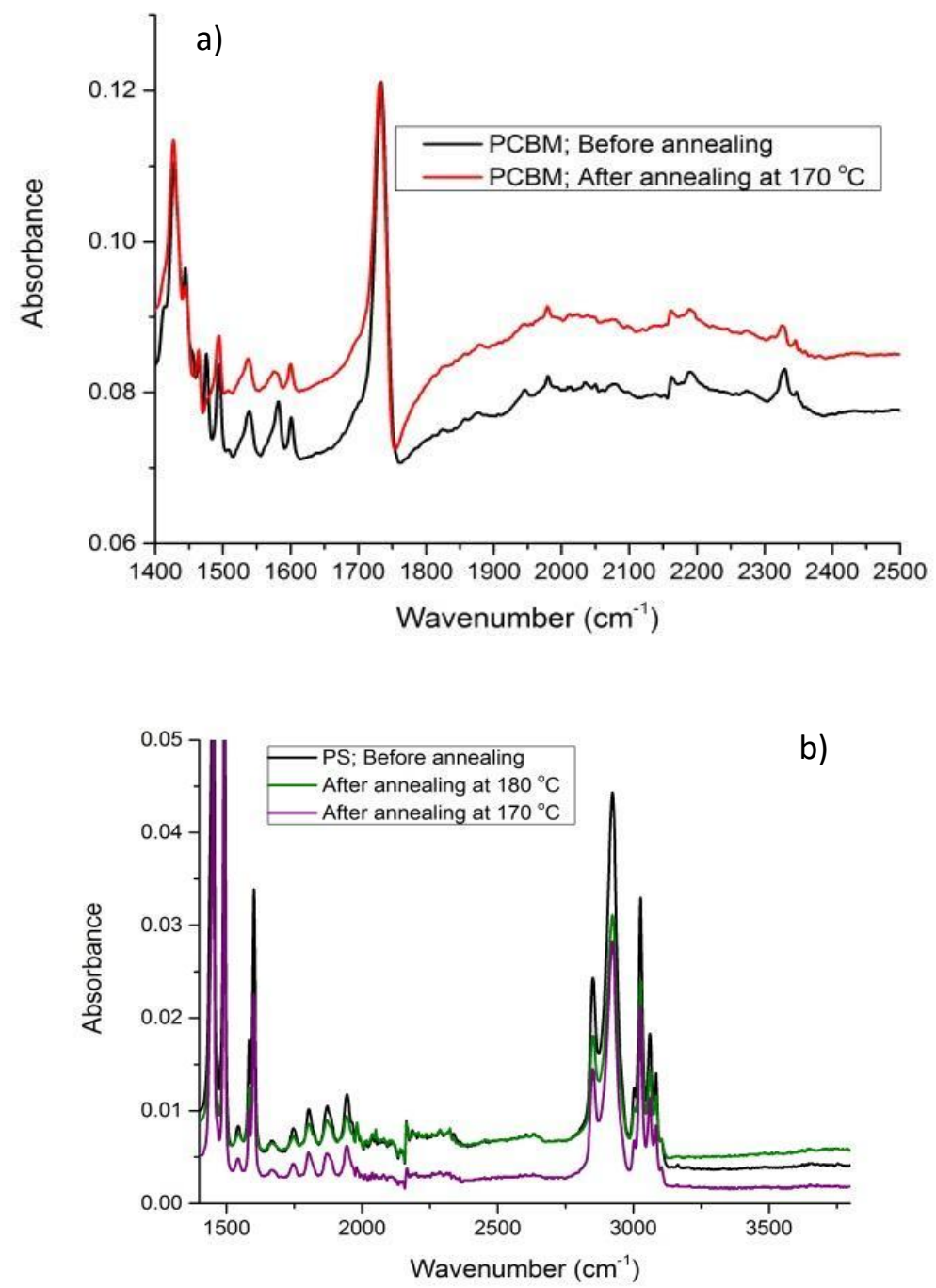

Figure 3.5-1: FTIR-ATR spectra of a) PCBM and b) PS films before and after annealing for 30 minutes at the temperatures shown, in a vacuum oven. The PCBM absorbance spectrum after annealing has been scaled by a constant factor so that the peak at $1737 \mathrm{~cm}^{-1}$ has the same height in the spectra before and after annealing. The force applied to the samples in all measurements was kept at a similar value. The differences in background level in the spectra before and after annealing are likely to be due to differences in the applied force pressing the sample onto the ATR crystal, and the resulting contact area between sample and AFM crystal. Especially in the case of PCBM in which the amount of material scraped onto the crystal was not rigorously controlled.

\subsection{Elastic Scattering}

Specular reflection is a tool used to measure composition profiles, frequently of polymer layers. A complete treatment of the theory presented in this section and 
sections 3.10 and 3.11 is contained in ' $\mathrm{X}$-ray and Neutron Reflectivity for the Investigation of Polymers' by T.P. Russell (Russell 1996), 'Elementary Scattering Theory' by D.S. Sivia (Sivia 2011) and 'Polymers and Neutron Scattering' by J.S. Higgins and H.C. Benoit (J. S. Higgins and Benoit 1994). Although there can be some inelastic scattering due to the motion of the sample molecules at any temperature above absolute zero, it is negligible in comparison to the elastic scattering. There is no transfer of energy between a static molecule and a scattered neutron. Layered samples on a substrate will scatter neutrons coherently, with an inplane coherence length of orders a few tens of microns(Sferrazza et al. 1997; Russell 1996). For neutrons it is usual to use samples that are larger than for x-rays to attain a strong signal, owing to the lower incident intensity of neutron sources, compared to synchrotron $\mathrm{x}$-ray sources. In elastic scattering (neutron or x-ray) we have the simplified case where the kinetic energy before and after the collision are equal. Particles incident with a wave vector $k_{i}$ emerges from the sample collision with wave vector $k_{f}$ (as in Figure 3.6-1) and the momentum transfer, $Q$, is defined a

$$
\boldsymbol{Q}=\boldsymbol{k}_{i}-\boldsymbol{k}_{f}
$$

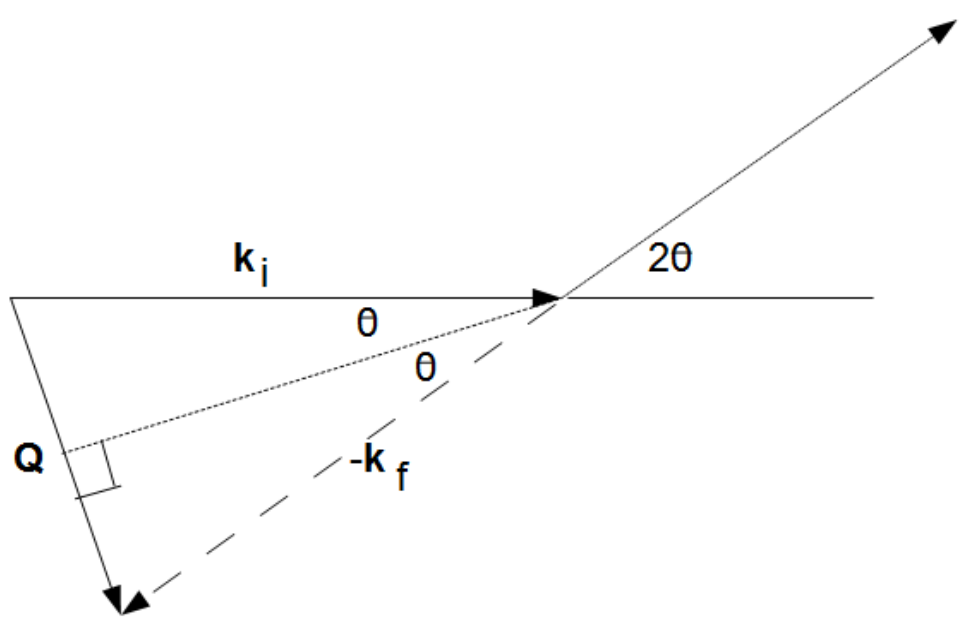

Figure 3.6-1: Geometry of momentum transfer $Q$ in terms of wave vectors. Doubleangle is used for convention. The plot shows the $x$-z plane, where the incident beam has its wavevector pointing along the $z$ direction.

As we are dealing with elastic scattering, the change in energy, $\Delta \mathrm{E}$, and the angular frequency associated with the scattering event, $\omega$, are both zero $\left(\Delta E=\frac{h}{2 \pi} \omega\right.$, where $h$ is Planck's constant). Hence 


$$
\left|\boldsymbol{k}_{i}\right|=\left|\boldsymbol{k}_{f}\right|=\frac{2 \pi}{\lambda}
$$

So the wavelength remains unchanged before and after scattering. From Figure 3.6-1 we infer using geometry that

$$
Q=\frac{4 \pi \sin \theta}{\lambda}
$$

This only a 2-dimensional relation so another angle, $\phi$, is needed to accommodate the third (y) axis which is normal to the $\mathrm{x}-\mathrm{z}$ plane such that

$$
\begin{gathered}
\boldsymbol{k}_{i}=\left(0,0, \frac{2 \pi}{\lambda}\right) \\
\boldsymbol{k}_{f}=\frac{2 \pi}{\lambda}(\sin 2 \theta \cos \phi, \sin 2 \theta \sin \phi, \cos 2 \theta) .
\end{gathered}
$$

This still satisfies $\left|\boldsymbol{k}_{f}\right|=2 \pi / \lambda$. The momentum transfer vector can be written

$$
\boldsymbol{Q}=\frac{4 \pi \sin \theta}{\lambda}(-\cos \theta \cos \phi,-\cos \theta \sin \phi, \sin \theta),
$$

which satisfies equation (3.9). This inverse relationship of wavelength to momentum transfer will be useful when it comes to analysing real data from neutron scattering experiments in later chapters. The incident flux, $\Phi$,of the incoming x-rays or neutrons is equal to their number per unit time per unit area.

Defining a function, $f(\lambda, \theta)$ describes the scattering amplitude that a particle of a given wavelength will scatter in a certain direction (called the scattering factor). For neutrons this tends to be invariant and gives

$$
f(\lambda, \theta)=-b
$$

where the constant $\mathrm{b}$ is called the scattering length (the minus sign is a convention). We take $f(\lambda, \theta)$ from the equation of a scattered wave from a single atom,

$$
\psi_{f}=\psi_{0} f(\lambda, \theta) \frac{e^{i k r}}{r}
$$

where we see that it is apt to call b a scattering length as it must have units of length. In principle scattering lengths are complex numbers, but the imaginary part is so 
small as to be negligible for the materials considered in this thesis. The scattering depends on the make-up of the nucleus and as such is isotope specific. It does not vary in any simple way, and indeed some values are negative. One practical use for this is in labelling by deuteration. Deuterium has a markedly different scattering length than hydrogen, and this fact can be used to contrast between parts of a sample (J. S. Higgins and H. C. Benoit 1994). Neutrons also have spin which gives them a magnetic dipole moment and causes them to interact with the dipole of the sample.

For X-ray scattering some of the principles of neutron scattering apply, and we still use the concept of the scattering factor, $f(\lambda, \theta)$. In this case, it is the long range electromagnetic force which causes the scattering and the properties of the scattering factor are different to that for neutron scattering. The scattering factor now diminishes with increasing $\theta$ and decreasing $\lambda$. It has the same sign for all the elements and is of magnitude proportional to the atomic number, $Z$, as

$$
f(\lambda, \theta)=Z g(Q) r_{e},
$$

where $r_{e}$ is the Thomson scattering length (the classical radius of the electron). Now we define the scattering cross section for both $\mathrm{x}$-rays and neutrons. The intensity of the reflected beam is given by the modulus squared of equation (3.14) with $\left|\psi_{0}\right|^{2}=$ $\Phi$, where $\Phi$ is the incident flux, giving

$$
\left|\Psi_{f}\right|^{2}=\Psi_{f} \Psi_{f}^{*}=\frac{\Phi}{r^{2}}|f(\lambda, \theta)|^{2}
$$

Now the scattering rate over all angles, $R$, is given by

$$
R=\int_{2 \theta=0}^{\pi} \int_{\phi=0}^{2 \pi}\left|\psi_{f}\right|^{2} d A
$$

The cross-section, $\sigma$, is defined as the scattering rate over the incident flux: $\sigma=$ $R / \Phi$.Which leads to the relationship

$$
\sigma(\lambda)=2 \pi \int_{2 \theta=0}^{\pi}|f(\lambda, \theta)|^{2} \sin 2 \theta d 2 \theta
$$

And so we get the relationship using equation (3.13) for neutrons, 


$$
\sigma=4 \pi|b|^{2}
$$

\subsection{Neutron and X-Ray Sources}

Neutrons and X-rays are both used in this thesis. The method of generating either one can have an effect on the counting times required to produce data with sufficiently small error bars. The source also dictates the experimental setup which will be described in detail at the end of this section. Neutrons can be produced two ways, spallation sources or fusion reactors. Spallation sources involve accelerating particles by a synchrotron and periodically colliding them with a target to produce neutrons. This produces pulses of neutrons. In fission reactors we have no synchrotron and a steady beam of neutrons. X-rays are also produced by synchrotrons.

Wiens Law tells us how the black body radiation of an object peaks in terms of temperature, $\mathrm{T}$, by

$$
\lambda_{\max }=\frac{2.90 \times 10^{-3}}{T} \mathrm{~m} .
$$

In the case of X-rays this formula predicts a temperature similar to that inside the Sun. Hence we resort to other ways of producing X-rays than blackbody radiation. Accelerating an electron through a potential $\mathrm{V}$ will emit a photon of wavelength $\lambda$ according to

$$
\lambda \leq \frac{h c}{e V} \approx \frac{1.24 \times 10^{-3}}{V} m
$$

Two mechanisms are at play here, Brehmsstralung radiation and an indirect process wherein the incoming electron knocks out an inner shell electron from the target which is followed by the relaxation of an electron by means of a transition from a higher energy level with the release of a photon. In this second process the energy and hence frequency, $v$, is given by

$$
\begin{gathered}
h v_{i j}=E_{j}-E_{i}, \\
\lambda_{i j}=\frac{h c}{E_{j}-E_{i}} .
\end{gathered}
$$


The dominant quantised energy is generally the transition from the second lowest energy level to the ground state and so that is the predominant wavelength at which most $\mathrm{x}$-ray work is done. Brehmsstralung occurs naturally in space and we see all sorts of X-ray spectra for galaxies and supernovae. X-ray synchrotrons typically have a radius of curvature on the order of $100 \mathrm{~m}$ depending on the number of beamlines.

In a synchrotron, electrons are travelling near to the speed of light and relativistic effects come into play. Recall the Lorentz factor, $\gamma$,

$$
\gamma=\left(1-\frac{v^{2}}{c^{2}}\right)^{-1 / 2} \gg 1
$$

Although radiation is being emitted in all directions, due to this Lorentz transformation the observer in the laboratory sees a narrow beam of $\mathrm{x}$-rays being emitted in the forward direction of the particle's motion. An observer in the plane of the orbit sees brief periods of emission of duration $\Delta t$,

$$
\Delta t \approx \frac{1}{2 \gamma^{2}} \times\left(\frac{\frac{1}{\gamma}}{2 \pi}\right) t,
$$

and

$$
t \approx \frac{2 \pi R}{c}
$$

givig

$$
\Delta t \approx \frac{R}{2 c \gamma^{3}}
$$

where the $2 \gamma^{2}$ factor is the Doppler factor for relativistic speeds. This process gives a continuous spectrum of photons with a lower wavelength bound, $\lambda_{\min }$, and median wavelength bound, $\lambda_{c}$, given by

$$
\begin{gathered}
\lambda_{\min } \approx c \Delta t, \\
\lambda_{c} \approx \frac{4 \pi R}{3 \gamma^{2}} \approx \frac{0.56 R}{E^{3}} \AA .
\end{gathered}
$$


The electric field of the emitted photons lies in the plane of the orbit causing an intrinsic polarisation characteristic of synchrotron radiation. In practise a synchrotron is made up of curved and straight sections. In the straight sections devices called undulators and wigglers act on the electrons with magnetic fields of alternating vertical polarity to force the electrons to execute horizontal oscillations allowing the emissions to build up rather than flash periodically. Undulators have become the principle source of X-ray radiation due to perturbation of the trajectory being much smaller. They are more brilliant than the Sun's emittance which is around $10^{10}$, with bending magnets having a brilliance of $10^{15}$ and undulators having a brilliance of around $10^{20} \mathrm{~s}^{-1} \mathrm{mrad}^{-2} \mathrm{~mm}^{-2} / 0.1$ bandpass.

\subsection{Neutron Reflectometers and Instrumental Setup}

Returning to neutrons, already mentioned briefly are the different types of neutron source. Outside a nucleus a neutron decays in about fifteen minutes into a proton, electron and an antineutrino. This is a relatively stable lifetime for the purposes of neutron scattering. Neutrons can be produced by firing Helium-4 nuclei at Beryllium resulting in Carbon-12 and a neutron. The emission rates are typically then $10^{6}$ to $10^{8}$ neutrons per second which is too low. This was merely the first neutron emitting process discovered and a higher flux interaction is the fission reaction of enriched uranium,

$$
n+{ }^{235} U \rightarrow{ }^{236} U^{*} \rightarrow{ }^{134} \mathrm{Xe}+{ }^{100} \mathrm{Sr}+2 n .
$$

There are other fission paths also in play, altogether leading to the average production of 2.5 neutrons per event and allowing a chain reaction to occur in the reactor over long time periods with a flux of up to $10^{15}$ neutrons per second. The neutrons produced are too fast to use so are slowed down by a moderator made up of light elements resulting in a Maxwell-Boltzmann distribution of wavelengths (Sivia 2011).

The alternative to fission sources are spallation sources wherein a proton is accelerated by a synchrotron and fired at a heavy element target to release neutrons. In this method there is a burst of high energy material at the beginning of the pulse which is cut out by choppers before it reaches the sample as it could be destructive on impact. The choppers regulate the wavelength range allowed in the beam. 
A schematic diagram of one of the reflectometers (Figaro) used in this study is shown in Figure 3.8-1, where we see the choppers that limit the incident neutron beams to certain velocities to prevent faster neutrons from damaging the sample or frame overlap. This reflectometer is designed for horizontal surfaces. The detector measures both specular and off-specular data.

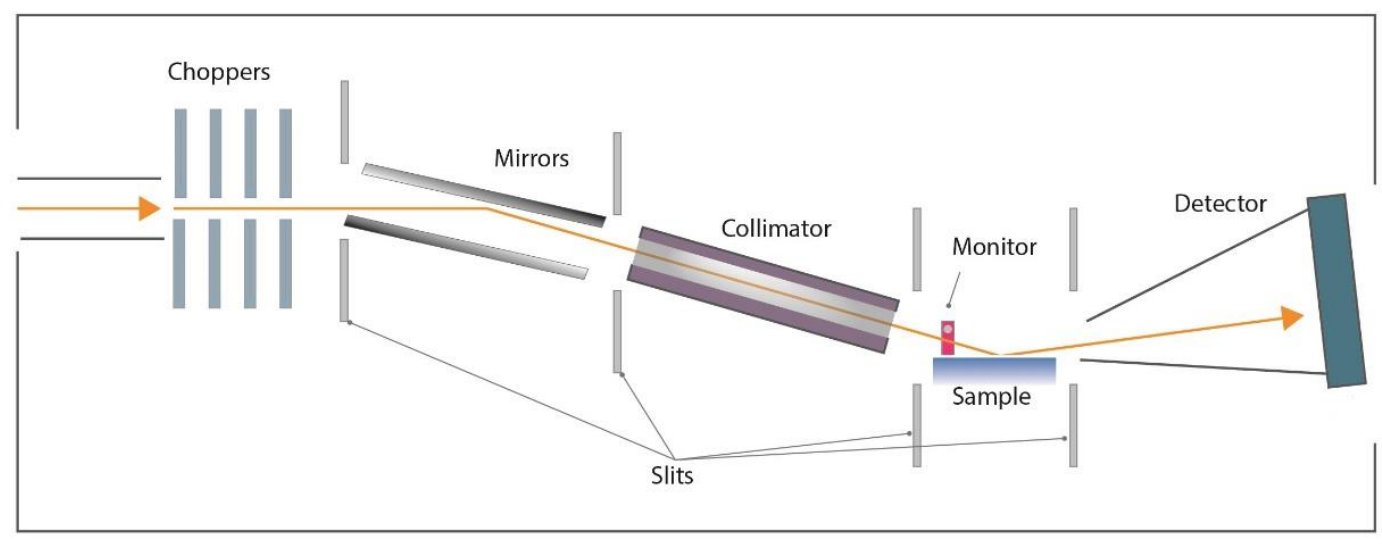

Figure 3.8-1: Detector setup at Figaro in the ILL for TOF mode. Reproduced with permission copyright Ruth Hynes.

Figure 3.8-1 shows a schematic diagram of Figaro, a time of flight (TOF) reflectometer. TOF is used for all NR measurements in this thesis. The neutrons can be generated either by nuclear decay (such as at the Institut Laue Langevin, Grenoble) or by accelerating protons in a synchrotron and colliding them with tungsten in spallation (such as at ISIS at the Rutherford Appleton Laboratory). The principle of TOF is to first measure the wavelength distribution of a collimated and chopped beam with no sample present (the direct beam). This is done by measuring the time taken for the neutrons to reach the detector and thus infering the momentum $\&$ hence wavelength of the neutrons. The intensity of neutrons reflected from a sample is then measured as a function of the wavelength (TOF) of the neutrons, and the reflectivity is calculated as the ratio of the intensity of reflected neutrons to incident neutrons.

Combining the curves obtained at different incident angles, is performed by using an instrumental scale factor for the intensity, matching the overlapping sections of the curves. In the particular case of INTER, two methodologies were used because of the greater consequence of any potential alignment error (due to the use of only a point 
detector at one angle). One of the data reduction methodolgies involved slight angle adjustment due to slight variation in the position of the critical edge, while the other methodology allowed no such adjustment in angle (and just used the incident angles determined during sample alignment). Both sets of reduced data were fitted. It was found that both sets of fits were very similar and the data shown from Inter in the thesis is from whichever method gave the lower chi squared value (for the fits to the data) for that sample.

Figaro has a 2D detector that allows for the collection of off-specular scattering and subtraction of a measured background from a strip of pixels parallel to the specular reflection. There is also a 2D detector at D17, at ILL, but a point detector at INTER, ISIS. At D17 the wavelength range can be selectively controlled by use of a double chopper system enabling a broad q-range to be measured in less than a minute.

For the neutron reflectivity data, three reflectometers were used: Figaro and D17 at the ILL and INTER at ISIS. For the data measured at Figaro the instrumental resolution was $2.9 \%$ to $3.9 \%$ increasing with q, $5 \%$ was used for all these samples in the fitting procedure. As well as using a constant dq/q resolution, a selection of the Figaro and D17 samples were also fitted with the resolution set equal to the (varying) instrument resolution from the data file (given above). These fits gave a higher value of chi-squared for all selected samples, and therefore all fits and fit parameters presented in this thesis are those with constant (fixed \%) resolution. The fit parameters themselves were very insensitive to changes in the resolution; in going from constant to varying dq/q resolution the Figaro and D17 layer SLD parameters changes by a maximum of $0.05 \times 10^{-6} \AA^{-2}$ and the interface roughness by a maximum of $1.2 \AA$ A. At Figaro two incident angles were used; neutrons were counted for ten minutes at the lower incident angle of $0.72^{\circ}$ and 75 minutes at the higher angle of $2.71^{\circ}$. At D17 the first and second angle of $0.2^{\circ}$ and $3.21^{\circ}$ were measured for 15 and 80 minutes with resolution of $1.7 \%$ to $5.8 \%$ increasing with q, and the kinetic samples were measured over a lower angle of $0.87^{\circ}$ for 30 seconds with resolution of $3 \%$ to $7.3 \%$, and were measured up to $q=0.1$. At INTER the first and second angle of $0.5^{\circ}$ and $2^{\circ}$ were measured for 15 and 45 or 90 minutes respectively. The samples were under illuminated for both the D17 and Figaro measurements and overilluminated at INTER. The instrument resolution for INTER of $2.6 \%$ does not fit the 
data so well so the resolution used in fitting was $3.5 \%$ for INTER. The fitted resolution for the D17 samples was $4 \%$. The key point is that in all cases the instrumental resolution parameter used in fitting the data, was fixed for the entire batch of samples measured on each instrument. For both the D17 and Figaro measurements, background was taken from a strip of pixels parallel to the specular reflection and subtracted from the data. This is not unreasonable as there was no significant off-specular scattering to interfere with the background measured at these pixels. The detector at INTER was linear so this was not possible, and no background was subtracted. The beam at INTER is a ribbon beam and the detector integrates across the width of the beam, while at the ILL the beam is also a ribbon but measured using an area detector where the data is measured at multiple parallel strips. The angle on the detector at which the highest intensity signal occurs, is used to form the reflectivity curve, with strips of pixels parallel to the specular reflection being used to measure background and any off-specular scattering. A small (constant) background of $1 \times 10^{-7}$ was included in the fits of the Inter samples, and the sensitivity to the inclusion or absence of background was probed for the extracted fit parameters (from measurements at all 3 instruments). It was seen that it made very little difference) A list of which samples were measured at which reflectometers is given in the Appendix. Errors of the fitted parameters are given by the standard deviation of the mean from groups of samples (either 'duplicate' samples, repeat measurements of the same sample on different instruments, or sets of closely related samples.

\subsection{Grazing Incidence X-ray Diffraction}

X-ray diffraction can be used to probe the state of order in a thin film. The x-rays are diffracted by repeated structures such as interatomic spacing in an amorphous material or a crystalline structure. Amorphous materials have localised ordering over short length scales but the pattern diminishes over longer length-scales. X-rays have very short wavelengths suitable for probing short length scales. When the angle of incidence is slightly less than the critical angle total reflection occurs at the surface and an evanescent wave is formed that penetrates about 5-10nm into the samples. When the incident angle is slightly bigger than the critical angle, the penetration 
depth is only limited by the absorption of the material. This is on the order of $10^{5} \mathrm{~nm}$ normal to the surface for organic materials which are typically in the form of amorphous glasses.

To understand the interaction of $\mathrm{x}$-rays with amorphous materials, take the radial distribution function. The autocorrelation function (ACF) of the scattering length density of the particles, $\beta$, can be written as the sum of two components as

$$
A C F[\beta(r)]=g_{1}(r)+g_{2}(r) .
$$

Here $g_{1}$ describes the interaction of the atom with itself and has a maximum at the origin. It decays rapidly over the atomic distance. The autocorrelation function is a measure of the distance distribution of structures in $\mathrm{f}(\mathrm{x})$ such that $A C F(x)=$ $\int_{-\infty}^{\infty} f(t) * f(x+t) d t$. The second term, $g_{2}$, describes the spatial distribution of the atoms and therefore the structure of the sample. It is spherically symmetric for liquids and amorphous materials so that

$$
\begin{aligned}
& g_{2}(-r)=g_{2}(r)^{*}, \\
& g_{2}(\boldsymbol{r})=g_{2}(r) .
\end{aligned}
$$

This is due to the particles inhabiting all orientations. The radial distribution function integrated over spherical coordinates, $\mathrm{P}(\mathrm{r})$, will be

$$
P(r)=4 \pi r^{2} g_{2}(r)
$$

It can be shown that this symmetric function gives rise to concentric rings on the detector map whose positioning indicate the atomic spacing of the molecule (Sivia 2011). Due to the evenly radially distributed disorder in the amorphous material, there is no preferred direction of refraction and a ring is formed at a constant angle determined by the molecular spacing.

In a crystal, the unit cells for each crystal are ordered in sheets. The path difference for waves reflected from successive sheets determines the intensity of the diffracted beam: a path difference equal to a whole number of wavelengths will produce a maximum intensity through constructive interference. The condition for constructive interference is the Bragg condition relating the angle of incidence, $\theta$, the spacing $\mathrm{d}$ and the wavelength $\lambda$ which is

$$
n \lambda=2 d \sin \theta
$$


where $n$ is an integer. Here $\sin \theta>1$ is impossible hence $d<\lambda / 2$. The crystal forms a three-dimensional diffraction grating which requires three integers to describe the order of the diffracted waves; $h, k$ and 1.

The periodicity can be described as

$$
\beta(r)=\beta\left(r+n_{1} a+n_{2} b+n_{3} c\right)
$$

where $n_{1}, n_{2}$ and $n_{3}$ are all integers. The length of the vectors $\mathrm{a}, \mathrm{b}$ and $\mathrm{c}$ and the angles between them are the lattice constants and the parallelepiped described by these is the unit cell that regularly repeats to form the crystal. The differential crosssection for elastic scattering for transfer momentum, Q, is

$$
\left(\frac{d \sigma}{d \Omega}\right) \propto\left|\iiint_{V} \beta(r) e^{i Q \cdot r} d^{3} r\right|^{2}
$$

There is a summation of an imaginary exponential over $n_{1}, n_{2}$ and $n_{3}$ in the Fourier transform which integrates over a unit cell which will cancel out to zero unless the terms coherently add up. This brings about the condition that

$$
Q \cdot\left(n_{1} a+n_{2} b+n_{3} c\right)=\phi_{0}+2 \pi n \text {. }
$$

$\phi_{0}$ is a constant and we get coherence when

$$
Q=h A+k B+l C,
$$

For $\mathrm{h}, \mathrm{k}$ and $\mathrm{l}$ being integers and $\mathrm{A}, \mathrm{B}$ and $\mathrm{C}$ being functions of $\mathrm{a}, \mathrm{b}$, and $\mathrm{c}$. The separation of consecutive sheets is given by

$$
d=\frac{a / 2}{\sqrt{\left(\frac{h}{m}\right)^{2}+\left(\frac{k}{m}\right)^{2}+\left(\frac{l}{m}\right)^{2}}},
$$

where $a$ is the edge length of a unit cell and $m$ is the wavelength path difference between sheets. In observing a peak the momentum vector is then parallel to the unit vector normal to the planes:

$$
Q \| \widehat{n} .
$$

GIXD measurements were performed on a synchrotron beamline at Diamond Light Source, at the Rutherford Appleton Laboratory, and on a lab based beamline at Sheffield University. At Sheffield a Xeuss 2.0 SAXS/WAXS laboratory beamline was used using a liquid Gallium MetalJet (Excillum) x-ray source (9.2 keV, $1.34 \AA$ ). The scattered X-rays were detected using a Pilatus3R $1 \mathrm{M}$ detector. The sample- 
detector distance was $306 \mathrm{~mm}$. The incident angle was $0.17^{\circ}$. At Diamond the I07 beamline was performed using $10.5 \mathrm{keV}$ or $1.1808 \AA \mathrm{x}$-rays with a sample-detector distance of $293 \mathrm{~mm}$ using a Pilatus $2 \mathrm{M}$ detector with incident angle $0-0.5^{\circ}$.

\subsection{Neutron Reflectivity: Weak and Strong Scattering}

For neutron scattering, consider a layered sample and ask how the reflectivity, R(Q), reveals information about the depth profile of the SLD. In a change with-respect-to Figure 3.6-1, the $\mathrm{z}$ direction is now the normal to the sample surface, and the SLD profile is given as $\beta(z)$. The elastic differential cross-section is proportional to the fourier transform of the SLD function (valid in the Born approximation where scattering is weak), as

$$
\left(\frac{d \sigma}{d \Omega}\right)_{e l} \propto\left|\iint_{V} \int \beta(\boldsymbol{r}) e^{i \boldsymbol{Q} \cdot \boldsymbol{r}} d^{3} r\right|^{2}
$$

As we are dealing with a finite sample of length $2 L_{x}$ in the $\mathrm{x}$ direction and length $2 L_{y}$ in the y direction we can evaluate this integral to

$$
\left(\frac{d \sigma}{d \Omega}\right)_{e l} \propto 16 \frac{\sin ^{2}\left(L_{x} Q_{x}\right)}{Q_{x}^{2}} \frac{\sin ^{2}\left(L_{y} Q_{y}\right)}{Q_{y}^{2}}\left|\int_{-\infty}^{\infty} \beta(z) e^{i z Q_{z}} d z\right|^{2}
$$

Here we have a product of two sinc functions corresponding to a diffraction pattern through a slit of size $\left(2 L_{x} \times 2 L_{y}\right)$ which has a maximum at $\left(Q_{x}, Q_{y}\right)=(0,0)$ of $16 L_{x}^{2} L_{y}^{2}$. Therefore for a layered sample most of the reflectance is specular and equation (3.43) reduces to

$$
\left(\frac{d \sigma}{d \Omega}\right)_{e l} \propto 16 L_{x}^{2} L_{y}^{2}\left|\int_{-\infty}^{\infty} \beta(z) e^{-i z Q} d z\right|^{2}
$$

With $Q_{z}=-Q$ as the z-axis is positioned on the surface of the sample.

With the interactions being elastic we have for $\mathrm{R}$

$$
R=\frac{\text { Rate of specular reflective scattering }}{\text { Rate of incidence }} .
$$




$$
\begin{gathered}
R(Q)=\frac{1}{4 L_{x} L_{y} \theta} \iint_{\Delta \Omega}\left(\frac{d \sigma}{d \Omega}\right)_{e l} d \Omega, \\
\iint_{\Delta \Omega}\left(\frac{d \sigma}{d \Omega}\right)_{e l} d \Omega \approx \frac{\Delta \Omega}{4} \times\left(\frac{d \sigma}{d \Omega}\right)_{e l} \text { and } \Delta \Omega \approx \frac{16 \pi^{2} \sin \theta}{L_{x} L_{y} Q^{2}}
\end{gathered}
$$

Using this approximation at $Q=(0,0,-Q)$ and equation (3.44) this leads to

$$
R(Q) \propto \frac{16 \pi^{2}}{Q^{2}}\left|\int_{-\infty}^{\infty} \beta(z) e^{-i z Q} d z\right|^{2}
$$

And by integration by parts

$$
R(Q) \approx \frac{16 \pi^{2}}{Q^{4}}\left|\int_{-\infty}^{\infty} \frac{d \beta}{d z} e^{-i z Q} d z\right|^{2}
$$

This highlights the $\mathrm{R}(\mathrm{Q}) \propto 1 / Q^{4}$ relationship which is clearly visible in the reflectivity from samples with sharp interfaces. This is one of the most basic properties between the inverse relationship of real space, $r$, and inverse space, $Q$.

In the quartic decay there are a series of fringes; Bragg and Kiessig fringes, which contain information on the layer thicknesses and internal structure (R. Kelsall, I Hamley 2005).

An exact optical treatment of a sharp interface, is given by the requirement that the wave is smooth and continuous over the interface; which means that $\psi$ and $d \psi / d z$ must be equal on either side.

The intensity, $\mathrm{T}$, of the transmitted wave is defined in the same way as the reflectivity and $R+T=1$. In medium 0 the total amplitude perpendicular to the surface is the sum of the reflected and incident waves.

$$
\psi(z)=\exp \left(i k_{0} \sin \theta_{0} z\right)+R^{1 / 2} \exp \left(-i k_{0} \sin \theta_{0} z\right) .
$$

The perpendicular component in medium 1 is then given by

$$
\psi(z)=T^{1 / 2} \exp \left(-i k_{1} \sin \theta_{1} z\right) .
$$

Equating these and the derivatives and solving for $\mathrm{R}$ gives

$$
R=\left|\frac{k_{0} \sin \theta_{0}-k_{1} \sin \theta_{1}}{k_{0} \sin \theta_{0}+k_{1} \sin \theta_{1}}\right|^{2}
$$

Recalling that $k$ is a function of $\lambda, R=R\left(\theta_{0}, \lambda\right)$ so that the reflectivity profile can be found by varying either parameter. In the measurements taken for this thesis the wavelength varied and two angles were measured to get a full $\mathrm{q}$ range from the 
critical edge to the lower q range of the same order of magnitude as the background. The position of the critical edge tells us about the refractive indices of the materials. The gradient directly after the critical edge tells us about the buried interfacial roughness. The gradient at high $\mathrm{q}$ tells us about the surface roughness and the periodicity of the fringes tells us about the layer thicknesses (J. S. Higgins and Benoit 1994).

\subsection{Neutron Reflectivity: Extracting fit parameters}

Neutron reflectivity is used to probe the depth profile of bilayers. This is possible because the neutron is neutrally charged so does not interact with the electron cloud of the atoms, only the nucleus, allowing it travel deeper into the sample. When a neutron beam is incident on a layered sample that only varies in composition normal to the substrate, the wave will be refracted and reflected so long as the refractive indices of the layers are different. The refractive index of a material, $\mathrm{n}$, is usually slightly less than 1 and is given to a good approximation by

$$
n=1-\delta+i \beta
$$

For neutrons the imaginary component accounts for absorbing media and is usually $0 ; \beta=0 . \delta$ is given by

$$
\delta=\frac{\lambda^{2}}{2 \pi} N_{A} \sum_{i} \frac{\rho_{i}}{A_{i}} b_{i}
$$

$b_{i}$ is the neutron scattering length of the ith component with density $\rho_{i}$, atomic weight $A_{i}$, $\lambda$ is the wavelength of the indicent neutrons and Avogadro's constant $N_{A}$. For polymers of monomer mass $M_{m o n}$ and the sum of the scattering lengths of the monomer, $b_{m o n}$,is given by

$$
\delta=\frac{N_{A} \rho \lambda^{2}}{2 \pi} \frac{b_{m o n}}{M_{m o n}}
$$

The scattering length density is output as a fit parameter, SLD, and is characteristic of the chemical makeup of the layer. The scattering length does not vary monotonically across the periodic table as it is isotope dependent as shown in Figure 3.11-1. SLD is given by

$$
S L D=\frac{\sum_{i}^{n} b_{i}}{V_{m}}
$$


$V_{m}$ is molecular volume which is a function of mass density.

At incident angles $\theta$ that are less than the critical angle $\theta_{c}$ there is total external reflection, therefore for the neutron beam to penetrate the samples it is required that $\theta>\theta_{c}$. It can be shown (Russell 1990) that

$$
\theta_{c}=(2 \delta)^{2}
$$

In vacuum, indicated by subscript 0 , the $\mathrm{z}$ component (defined as normal to the substrate) of the wave vector is given by

$$
k_{z, 0}=\frac{2 \pi}{\lambda} \sin \theta
$$

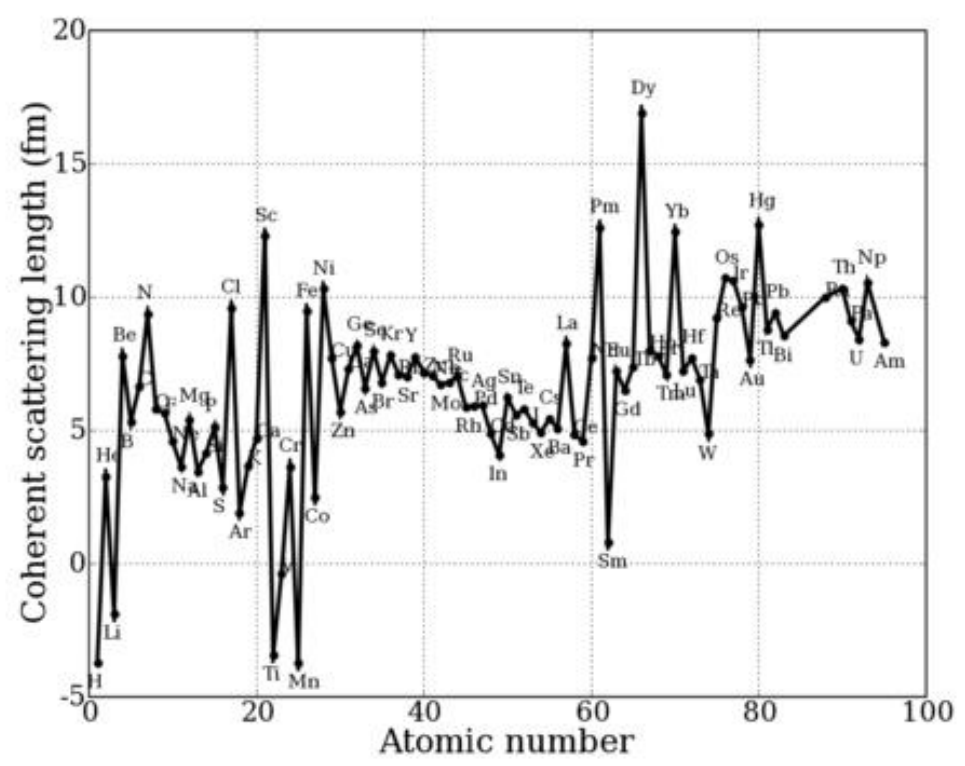

Figure 3.11-1: Scattering length values as a function of atomic number. Reproduced under the Creative Commons licence from reference (Gisaxs.com 2015).

This can also be described in terms of momentum transfer for specular reflection, $q$, which is easily verifiable from Figure 3.11-2, as

$$
\begin{gathered}
\boldsymbol{q}=\boldsymbol{k}_{f}-\boldsymbol{k}_{i}, \\
|\boldsymbol{q}|=q=\frac{4 \pi}{\lambda} \sin (\theta) .
\end{gathered}
$$




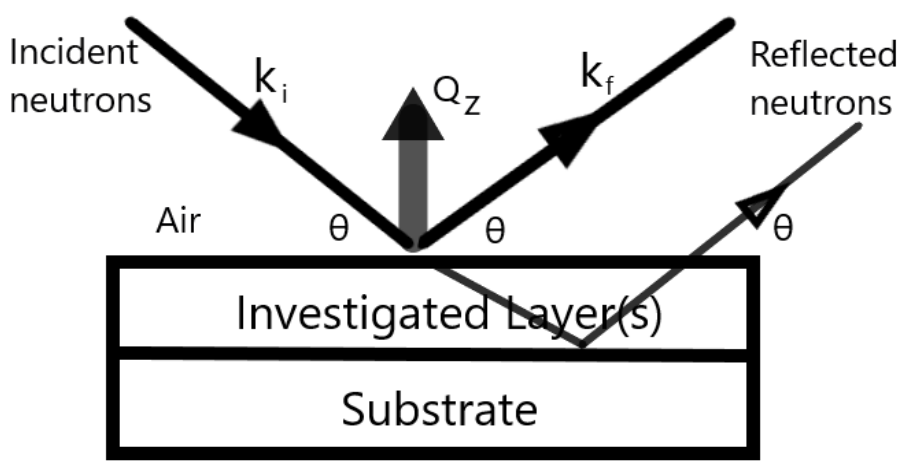

Figure 3.11-2: Diagram of geometric relation between $k$, $\theta$ and $Q$.

For specular reflectivity, the angle of incidence is equal to the angle of detection and for the nth layer

$$
k_{z, n}=\left(k_{z, 0}^{2}-k_{c, n}^{2}\right)^{1 / 2}
$$

Therefore the condition for specular reflection can be achieved by varying with the wavelength of the incident neutrons or the incident angle. For an arbitrary number of layers on the substrate, it is possible to calculate the total reflectance by iterating through the Fresnel reflectance of each layer, $r_{n, n+1}$, starting with the layer closest to the substrate, the interface between the nth and $(n+1)$ th, by

$$
r_{n, n+1}^{\prime}=\frac{k_{z, n}-k_{z, n+1}}{k_{z, n}+k_{z, n+1}}
$$

The Fresnel reflectance between the nth and (n-1)th layer, of thickness $d_{i}$, is given by

$$
r_{n-1 . n}=\frac{r_{n-1, n}^{\prime}+r_{n, n+1}^{\prime} \exp \left(2 i d_{n} k_{z, n}\right)}{1+r^{\prime}{ }_{n-1, n} \exp \left(2 i d_{n} k_{z, n}\right)}
$$

Equation (3.63) is iterated up through the layers by replacing n-1 with n-2 and n with n-1 until the sample/air interface reflectance, $r_{0,1}$, is reached (Russell 1996). The Fresnel reflectivity, $\mathrm{R}$, is then given by

$$
R=r_{0,1} r_{0,1}^{*}
$$

Total external reflection occurs below $k_{c}$ as in Figure 3.11-3. For a bare silicon substrate with an infinitely sharp interface, the reflectivity obeys $R \propto\left(\frac{k_{c, 1}}{k_{z, 0}}\right)^{4}$ at high values of $k_{z, 0}$. 

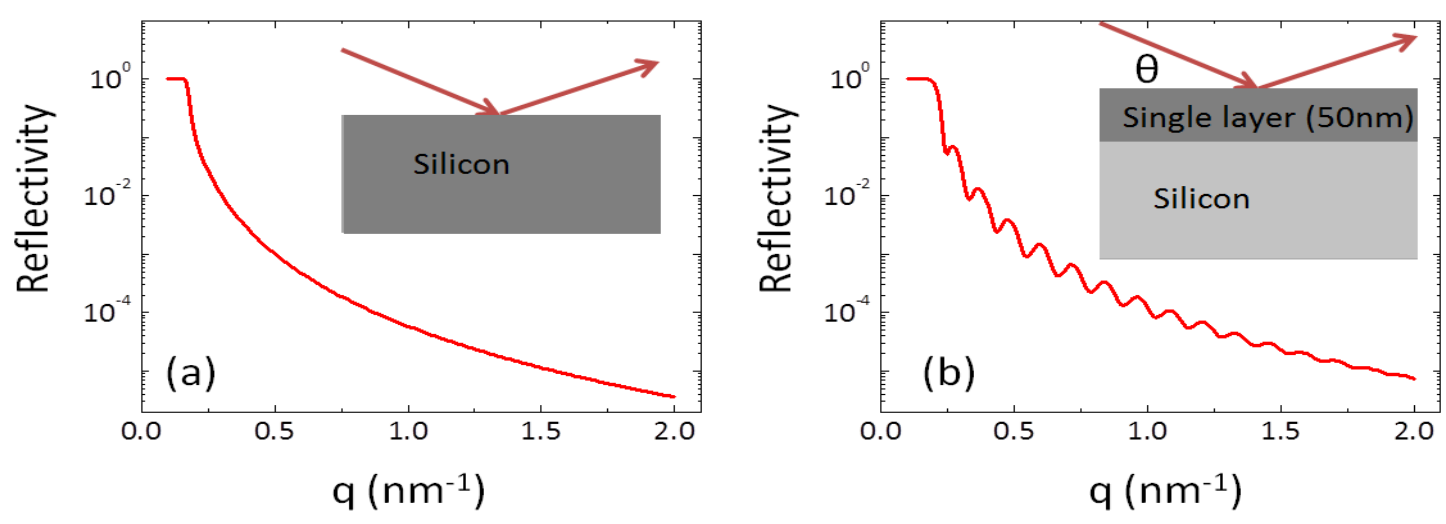

Figure 3.11-3: Reflectivity curves for a bare silicon substrate (a), and a silicon substrate with a 50nmlayer on top $(b)$, adapted with permission from reference $(X$. Zhang et al. 2016).

However, a diffuse interface causes the reflectivity to fall off more rapidly and dampens the fringes observed that describe the width between each interface. Where the exponential in equation (3.63) is multiplied by its complex conjugate we obtain real values for the reflectivity in the form of a cosine with the argument of film thickness and wave vector. Differentiation of this yields a sine function with successive minima at even multiples of pi, giving the thickness of a single layer, for example, directly as

$$
d=\frac{\pi}{\Delta k_{z, 1}}
$$

Each layer contributes a group periodicity in the observed reflectivity profile as well as smaller fringes (due to being from a larger distance between the surfaces in the sample) that describe the overall thickness of the sample. Knowing the thicknesses by using other techniques, such as AFM, enables corroboration of the fitted profile parameter values. The above is for infinitely sharp interfaces between the layers. In the case of a rough interface, the scattering length density (SLD) profile can be modelled as a number of very thin layers with gradually changing SLD values for each thin layer. It is also possible to account for this rough interface by applying a Gaussian convolution to the reflectivity. The roughness measured is dependent on the coherence length of the neutron beam and the size scale over which lateral differences in the roughness can be measured. For instance, a surface profile measured over two length-scales with the same average roughness but different profiles will give the same result if both length-scales are less than the coherence 
length. The effect of a wavy surface that modifies the incident angle is indistinguishable from divergence of the beam.

The smearing function in the z-direction for standard deviation from the average position of the interface, $\sigma$, is given by

$$
G(z)=\frac{1}{(2 \pi)^{1 / 2} \sigma} \exp \left(\frac{-z^{2}}{2 \sigma^{2}}\right) .
$$

The reflectivity for infinitely sharp interfaces will be denoted as $R_{F}$ and the smearing function can be applied (for a single interface) as

$$
R\left(k_{z, 0}\right)=R_{F}\left(k_{z, 0}\right) \exp \left(-4 k_{z, 0}^{2} \sigma^{2}\right)
$$

For more than one interface, the reflectance from each interface is multiplied by an exponential function of the roughness at each interface (Russell 1996; R. A. L. Jones and R. W. Richards, 1999). This has the effect of damping the fringes but has no effect on the spacing of the fringes so the previous relation in equation (3.65) holds true. The roughness of different interfaces can be distinguished if they are considerably different from one another as it can be shown that they will case damping at different rates across q (Russell 1990).

As mentioned previously, the roughness of the interface measured in this way includes both the molecular mixing at the interface between the phases, and lateral non-uniformity (lateral roughness) of the sample surface (due to the capillary waves at a liquid interface). It is possible to distinguish between these by taking offspecular measurements. For laterally rough samples there will be a Yoneda peak as shown in Fig 3.11-4.

This shows the scattering from one particular sample studied by James et al. In this study a range of polymer/polymer bilayer samples were measured, of differing bottom layer thickness. Fitted values of the lateral roughness at the buried interface in these samples ranged from $10 \AA$ to $25 \AA$, with all of these samples showing extensive off-specular scattering. 

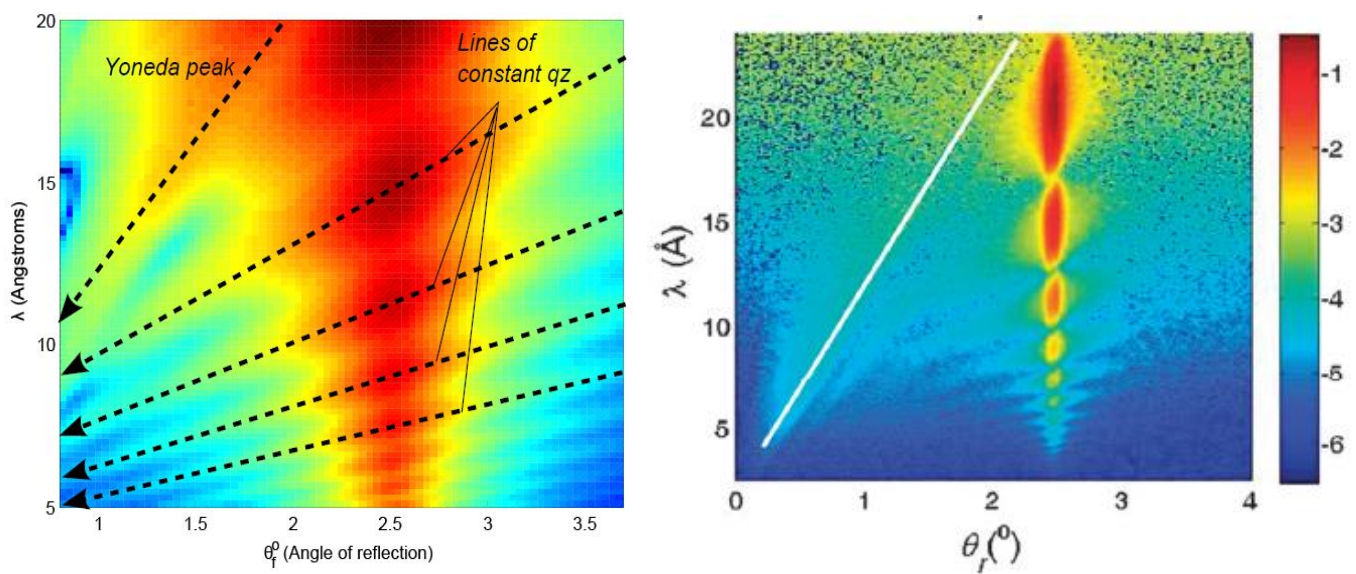

Figure 3.11-4: A model detector map (left) showing the location of a Yoneda peak in relation to the specular scattering seen for $\theta=2.5$, reproduced with permission from reference $(D . W$. James 2011). Specular and off-specular neutron reflectivity (right) measured from an annealed polymer/polymer bilayer on a silicon substrate. Sample characteristics on this sample were: bottom layer had an SLD of $6.83 \times 10^{6}$ and a thickness of $480 \AA$, the top layer had an SLD of $5.3 \times 10^{-7}$ and a thickness of 1000A. These were measured by a combination of AFM measurements and fits to the specular and offspecular scattering. The lateral roughness at the silicon/bottom layer was $3 \AA$ or $4 \AA$ ( $3 \AA$ is the fit to the NR data, and $4 \AA$ is the rms measurement from AFM). The total roughness at the buried polymer/polymer interface in this sample was $22 \AA$ (consisting of $15 \AA$ lateral roughness and $16 \AA$ intrinsic roughness). Reproduced with permission from reference (D. James et al. 2015).

\subsection{Temperature callibration}

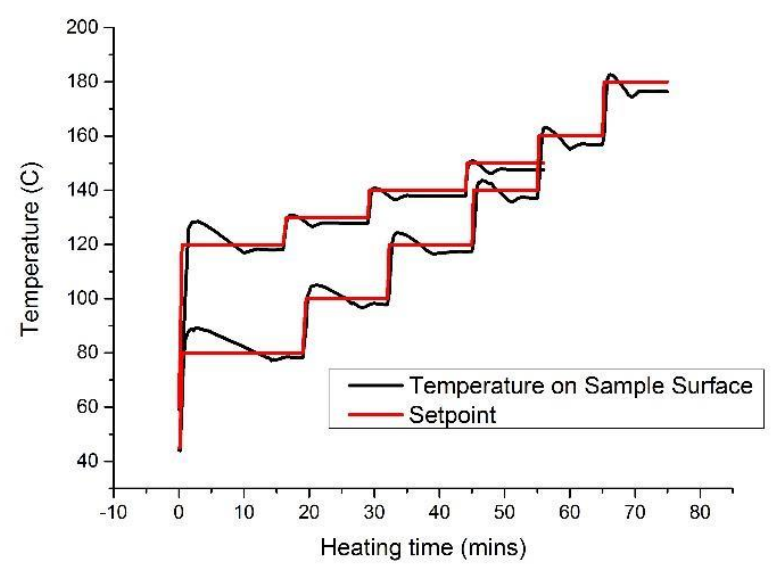

Figure 3.12-1:Setpoint of heater and temperature on sample surface, for a 2 inch diameter silicon wafer using the in-situ annealing procedure. Overshoots in the sample surface temperature are seen for jumps of 20 degrees (from $80^{\circ} \mathrm{C}$ to $180^{\circ} \mathrm{C}$ ) and 10 degrees (from $120^{\circ} \mathrm{C}$ to $150^{\circ} \mathrm{C}$ ). The error on the sample surface is $+/-1.5^{\circ} \mathrm{C}$ 


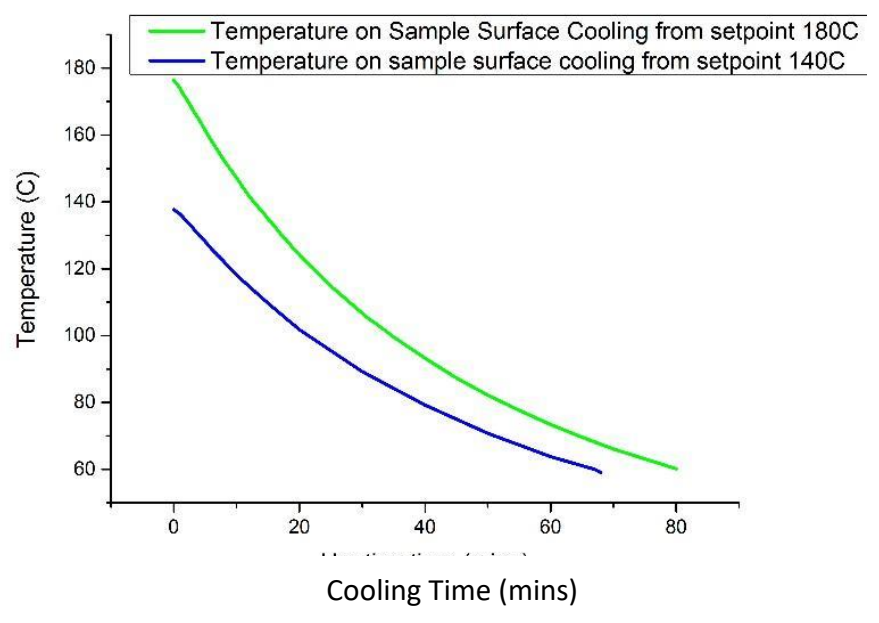

Figure 3.12-2: This graph shows the cooling of the sample surface from $180^{\circ} \mathrm{C}$ and $140^{\circ} \mathrm{C}$ to $60^{\circ} \mathrm{C}$ when the setpoint is dropped to $60^{\circ} \mathrm{C}$. Error on sample surface of $+/-$ $1.5^{\circ} \mathrm{C}$

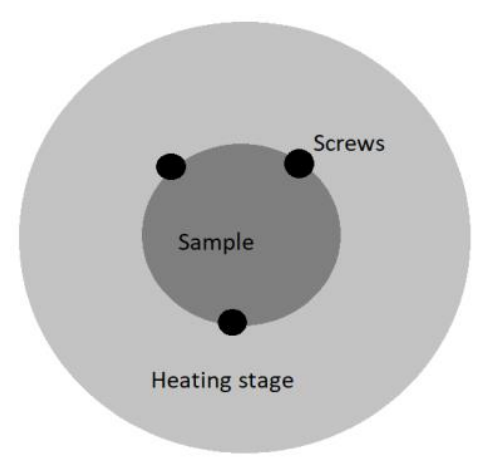

Figure 3.12-3: Schematic diagram of how the samples were attached to the stage during the in-situ measurements. 


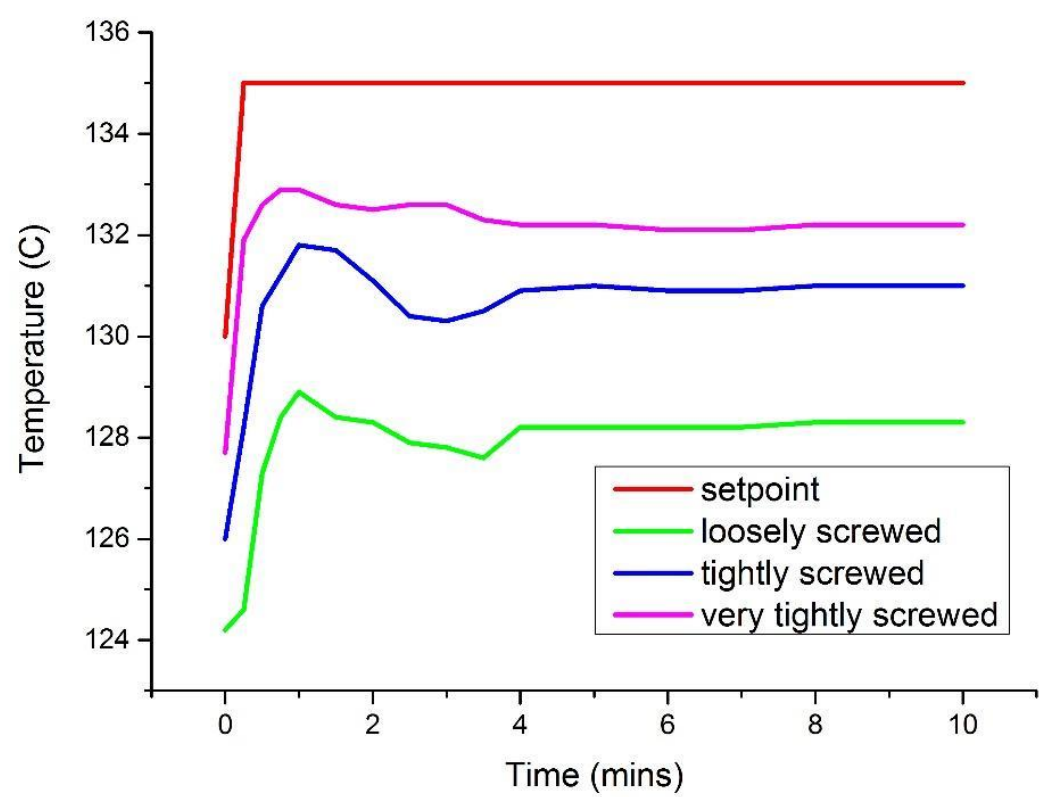

Figure 3.12-4: Demonstration of the dependence of the temperature on the sample surface on how tightly the sample is screwed onto the surface.

The ex-situ samples were heated in a vacuum oven and the in-situ samples were heated inside a vacuum chamber with a heating stage (in the neutron beam). The crystal study samples were heated using a Linkam heating stage under a dry nitrogen atmosphere. The temperatures quoted for the crystal study are from the thermocouple of the heating block in the Linkam stage. Temperature calibration at D17 for in situ samples as in Figure 3.12-1 and Figure 3.12-2 shows the setpoint and the temperature measured at the sample surface using a k-type thermocouple. For the calibration, the samples were screwed on very tightly, so after the experiment, the calibration shown in Figure 3-14 was performed which gives a more accurate error bar to the conditions of the experiment. The temperature overshoots the setpoint initially and then stabilises within five minutes. Figure 3.12-4 shows the offset between the setpoint and the sample surface temperature for variously tightly screwed samples. This is due to the fact that very tightly screwed samples bent slightly which distorted the reflectivity profile. During the in-situ annealing measurements, the samples were therefore screwed down to the heater in ways that were in-between the 'loosely' and 'tightly' attached cases shown in Figure 3.12-4. This resulted in a uncertainty in the sample surface temperatures of $+/-1.5$ degrees, which is within the accuracy of the thermocouple (which is $+/-2.2$ degrees). This 
error applies to the in-situ samples only, whose stabilised temperature offsets are shown in Table 1.

\begin{tabular}{|l|l|l|l|l|l|l|l|l|}
\hline Setpoint & 80 & 100 & 120 & 130 & 140 & 150 & 160 & 180 \\
\hline $\begin{array}{l}\text { Sample surface } \\
\text { temperature }\end{array}$ & 77 & 94 & 114 & 124 & 134 & 144 & 153 & 172 \\
\hline
\end{tabular}

Table 1: Setpoint and sample surface temperature for in-situ annealed samples.

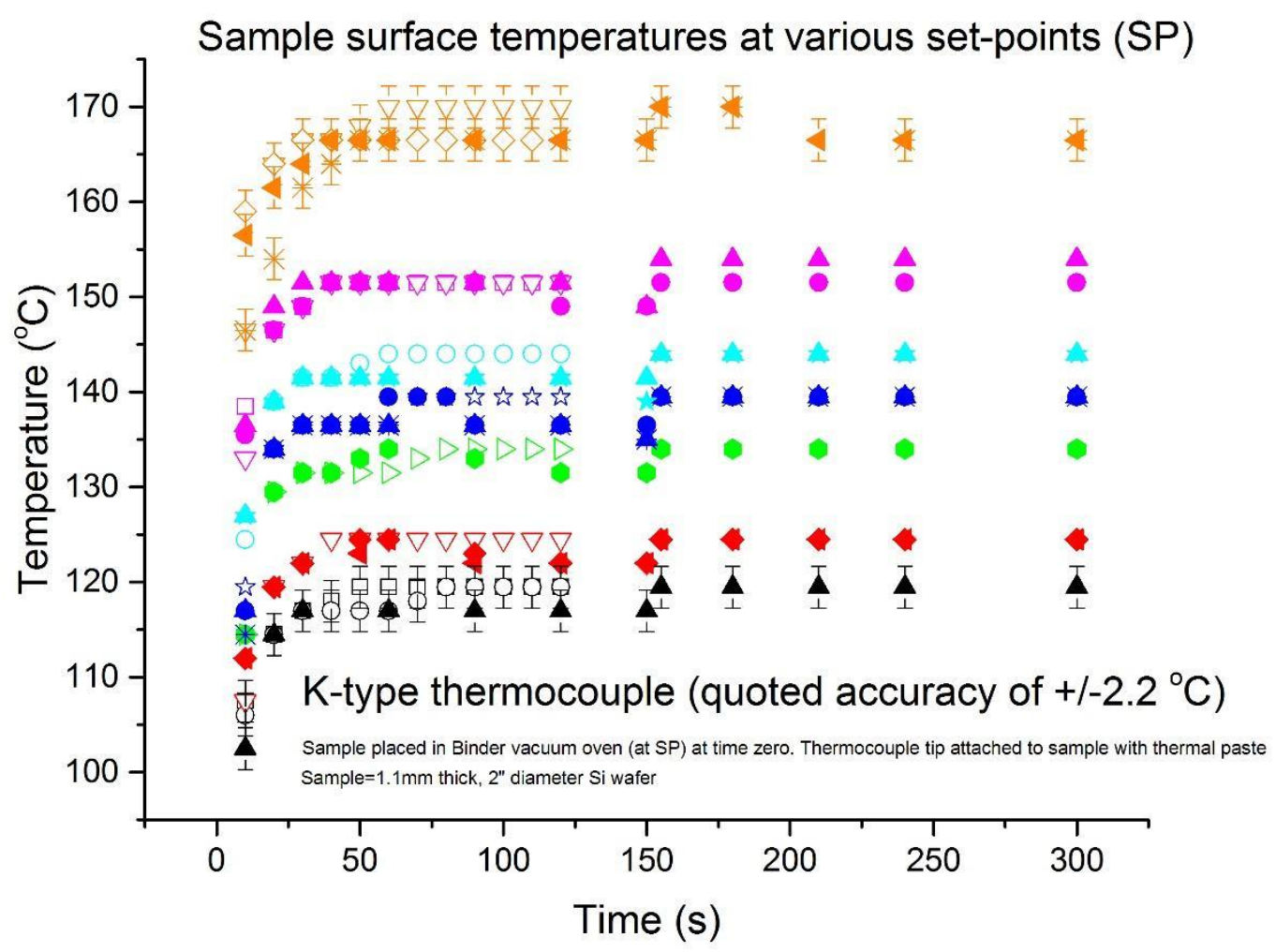

Figure 3.12-5: Calibration of vacuum oven for annealing temperatures used in exsitu samples (performed by Anthony Higgins, Swansea University).The different colours represent the different set-points given in Table 2 (in ascending order from red to orange). Different symbols represent repeat measurements (including at slightly different locations within the oven)

The offsets of the ex-situ samples annealed in the vacuum oven were similarly calculated using a thermocouple attached to a silicon sample. The vacuum oven was left for hours to stabilise at the set-point temperature, and the silicon sample with a thermocouple attached to its surface, was placed in the oven and the vacuum pump started at time zero. The sample surface temperatures versus time are shown in 
Figure 3.12-5, and the average stabilised sample surface temperatures are shown in Table 2 with an error of the thermocouple of $+/-2.2$ degrees.

\begin{tabular}{|l|l|l|l|l|l|l|l|}
\hline Setpoint temperature $\left({ }^{\circ} \mathrm{C}\right)$ & 120 & 125 & 135 & 140 & 145 & 155 & 170 \\
\hline Sample surface temperature $\left({ }^{\circ} \mathrm{C}\right)$ & 117 & 122 & 132 & 139 & 142 & 152 & 167 \\
\hline
\end{tabular}

Table 2: Setpoint and sample surface temperature for ex-situ annealed samples in the vacuum oven.

Bearing in mind the potential sensitivity of the sample behaviour to the temperature a range of annealing temperatures above the glass transition were explored. Further, the in-situ experiments give reason to believe that the annealing times chosen (1minute $[1 \mathrm{~m}]$ to $10 \mathrm{~m}$ and usually $5 \mathrm{~m}$ ) are sufficiently long for the system to reach equilibrium without the formation of crystals in the case of the PCBM bottom layer.

All the temperatures quoted are the nominal setpoint temperature unless otherwise stated and the molecular weights are the nominal values unless otherwise stated. 


\section{Results and Discussion}

\subsection{The impact of molecular weight on the mixing and interfacial width in PCBM/PS bilayers}

In this chapter, the data from NR experiments on thermally annealed PCBM/PS bilayers is presented and discussed. The molecular weight, annealing temperature and annealing time were varied to see the effect on the measured reflectivity and fitted composition profile. As detailed in chapter 2, the thermodynamics of mixing and interface formation of the benchmark fullerene derivative, PCBM, and the wellcharacterized, atactic polymer, PS will be probed. It is of use in the design of efficient OPV devices (Spanggaard and Krebs 2004). Molecular weight has a potentially complex effect on OPV devices (Ballantyne et al. 2008; Spoltore et al. 2015), so this model, low-polydispersity polymer system was used to probe the fundamental science occurring in polymer nanocomposite films.

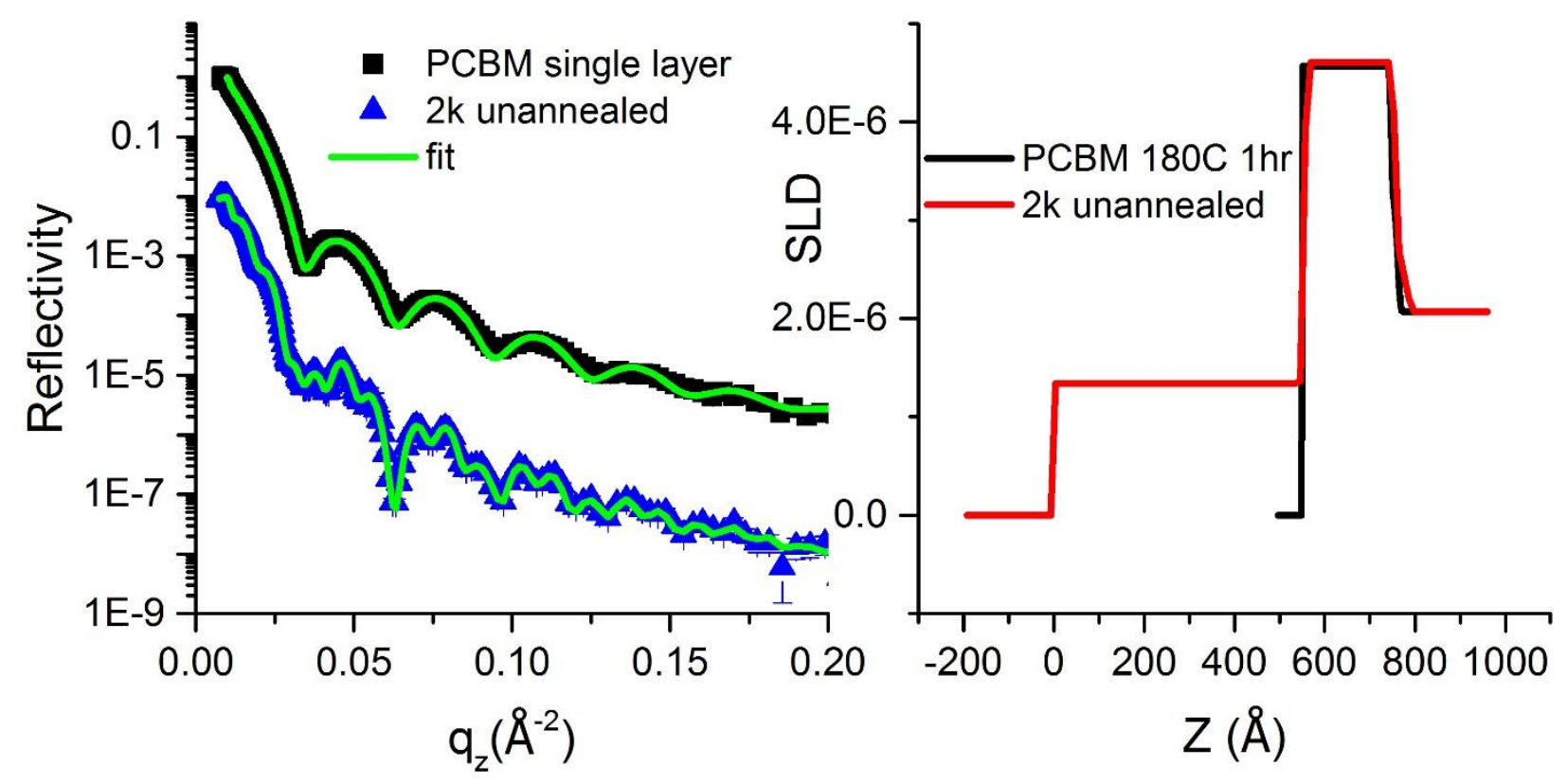

Figure 4.1-1:NR curves (left) and SLD profiles (right) for PCBM single layer and unannealed $2 k$ PCBM/PS bilayer.

Figure 4.1-1 shows the NR curve for a single layer of PCBM annealed at $180^{\circ} \mathrm{C}$ for one hour on a silicon substrate. This corresponds to fitted parameters of $19.8 \mathrm{~nm}$ for 
the thickness of the layer and an SLD value of $4.56 \times 10^{-6} \AA^{-2}$. This corresponds reasonably closely with the literature value (Clulow et al. 2014) of $4.66+/-0.23$ $\times 10^{-6} \AA^{-2}$ where there is some spread in the reported values found by neutron experiments. The value calculated by the NIST calculator for a density of $1.5 \mathrm{~g} / \mathrm{cm}^{3}$ (Sun, Han, and Liu 2013) is $4.34 \times 10^{-6} \AA^{-2}$. The errors in this thesis are taken as the standard error of the mean except for the error bars on the NR curves which are the standard deviation of the Poisson distribution.

Several unannealed bilayers consisting of pure PS layers, of various MW, on top of pure PCBM were measured by NR. A typical example is shown in Figure 4.1-1 The NR curves, fits and SLD profiles for the remaining unannealed samples are presented throughout this chapter, but they all show a consistent bilayer SLD profile with the following average fit parameters; PCBM SLD $=4.65+/-0.02 \times 10^{-6} \AA^{-2}$, PS SLD $=1.31+/-0.035 \times 10^{-6} \AA^{-2}$, Gaussian interface roughness $=6.41+/-1.86 \AA$, surface roughness $=5.1+/-2.98 \AA$. The large error for the surface roughness indicates that NR is not particularly sensitive to this parameter. The value for the SLD of PS according to the polymer handbook (Sinha and Buckley 2007) is $1.41 \times 10^{-6} \AA^{-2}$. The density of PS reported in Polymer Physics (R. Colby; M. Rubinstein; 2003) is $0.969 \mathrm{~g} / \mathrm{cm}^{3}$ which gives a theoretical SLD value of $1.303 \times 10^{-6} \AA^{-2}$. The values of SLD for pure PCBM and pure PS, used in this thesis are taken as the average of experimentally measured unannealed values.

In this chapter ex-situ and in-situ annealing measurements are presented. These methodologies are complementary. Ex-situ allows heating of a sample at a controlled temperature for a short time (typically 1-5 minutes), which enables control over sample quality. Rapid quenching of the ex-situ samples freezes in the morphology at elevated temperature, whereas in-situ annealing allows measurement of the composition as a function of temperature and to observe the effect of cooling. While ex-situ does not in principle allow a study of the effect of temperature, due to the potential for composition development during cooling, the two techniques can be compared directly. In-situ measurements allow us to probe the kinetics albeit with lower quality measurements due to the shorter counting times.

GIXD was performed on a subset of the samples to determine whether there was any significant crystallisation following annealing. Potentially extensive 
crystallisation at either crystal size scale could impact the measured roughness. In some cases we have rejected samples due to the presence of defects, some of which are seen to be crystals. GIXD rings are shown in the Appendix to this chapter.

\subsubsection{Ex-situ annealing of PS/PCBM bilayers 2K PS}

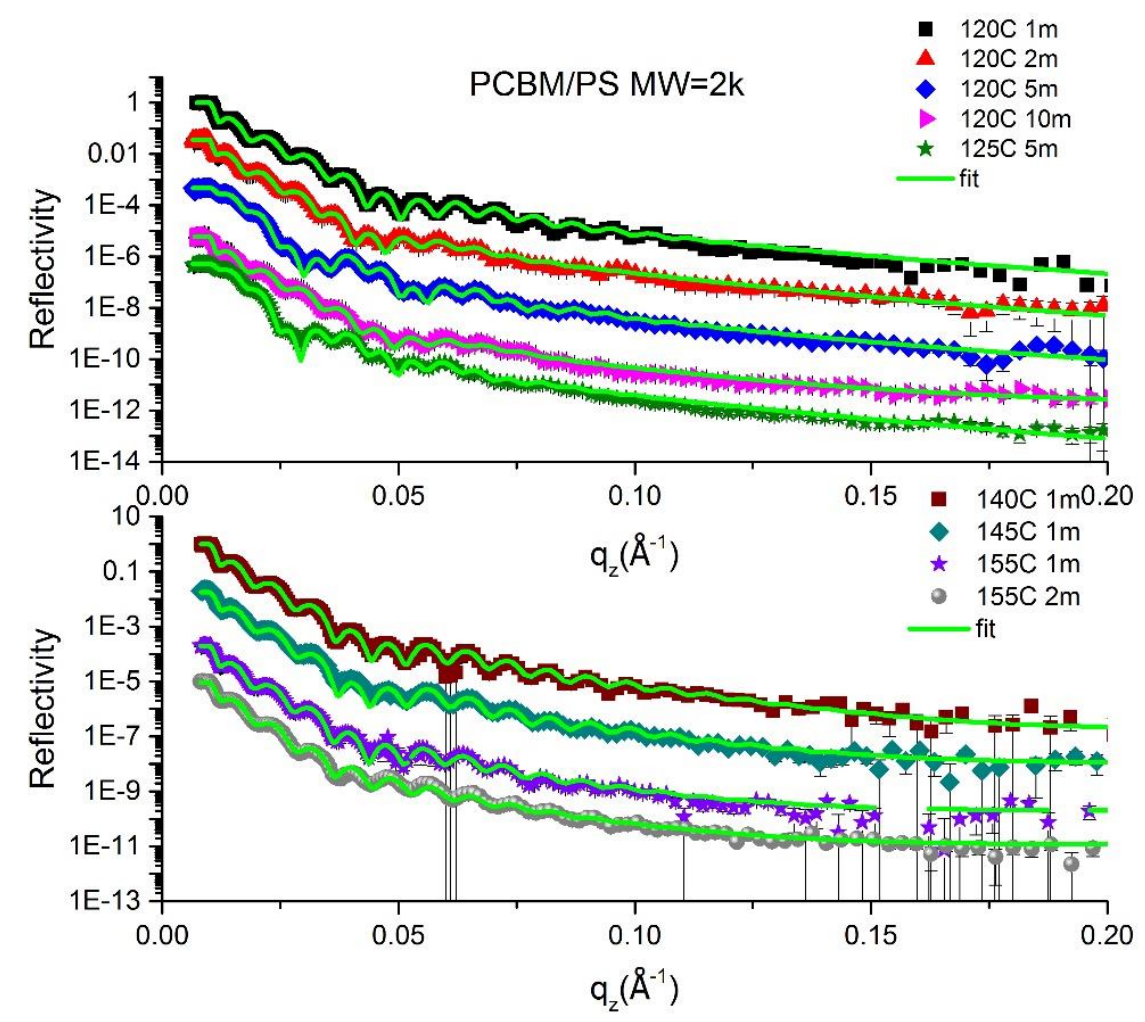

Figure 4.1-2: NR curves for PCBM/PS bilayers with PS $M W=2 k$ Da annealed at various temperatures. Curves are offset vertically for clarity in this and many subsequent reflectivity figures. 


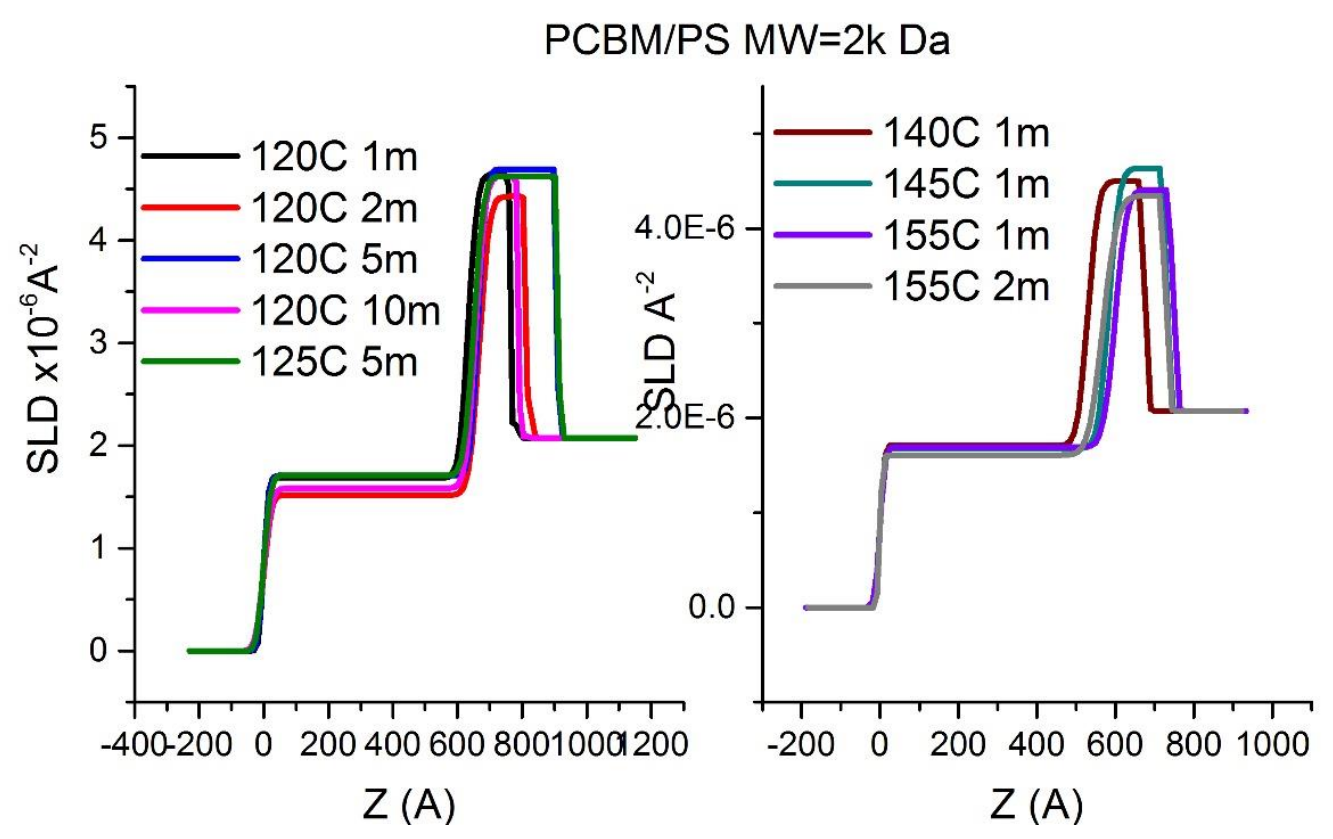

Figure 4.1-3:SLD profiles for PCBM/PS bilayers with PS MW=2k Da annealed at various temperatures.

Figure 4.1-2 and Figure 4.1-3 show the NR curves and SLD profiles for the annealed 2K PS/PCBM bilayers. The neutrons interfere to cause the observed NR curves with clear visible evidence that more than a single layer exists on top of the silicon substrate. Two periodicities are clearly seen, providing evidence of significant reflection from a buried interface between two layers. The fitted SLD profiles reveal an elevated SLD value for the top layer of $1.65+/-0.024 \times 10^{-6} \AA^{-2}$. This is significantly above the value for pure PS and is evidence of diffusion of some PCBM into the top layer. In contrast, the SLD of the bottom layer has not changed significantly following annealing (for the annealed 2K PS samples the bottom layer SLD of $4.53+/-0.042 \times 10^{-6} \AA^{-2}$ is unchanged within error, from the value before annealing). Consistent with such an increase in the top-layer SLD and a constant bottom layer SLD, is the thinning of the PCBM layer following annealing. All samples in Figure 4.1-2 show bottom (PCBM) layers that are considerably thinner than the starting thickness. The starting thickness was the same for 7 of the 9 samples (all except two, at 120C and 125C 5m, samples; all 7 PCBM layers were spin-coated from the same concentration of PCBM solution at the same spin-speed for the single layer and bilayers in Figure 4.1-1 and Figure 4.1-2 ). The starting bottom layer thickness of the $2 \mathrm{k}$ samples (taken from unannealed samples) was $21.3 \mathrm{~nm}+/-0.52 \mathrm{~nm}$ and the average thickness after annealing was $14.14 \mathrm{~nm}+/-$ $0.35 \mathrm{~nm}$. 
The gradient of the line joining the top and bottom layer in the SLD profile is also less steep in annealed bilayers than in the unannealed samples. This indicates a broader interfacial width. This broadening of the interface after annealing is seen in all the MW that were looked at.

\subsubsection{Probing the robustness of fit parameters}

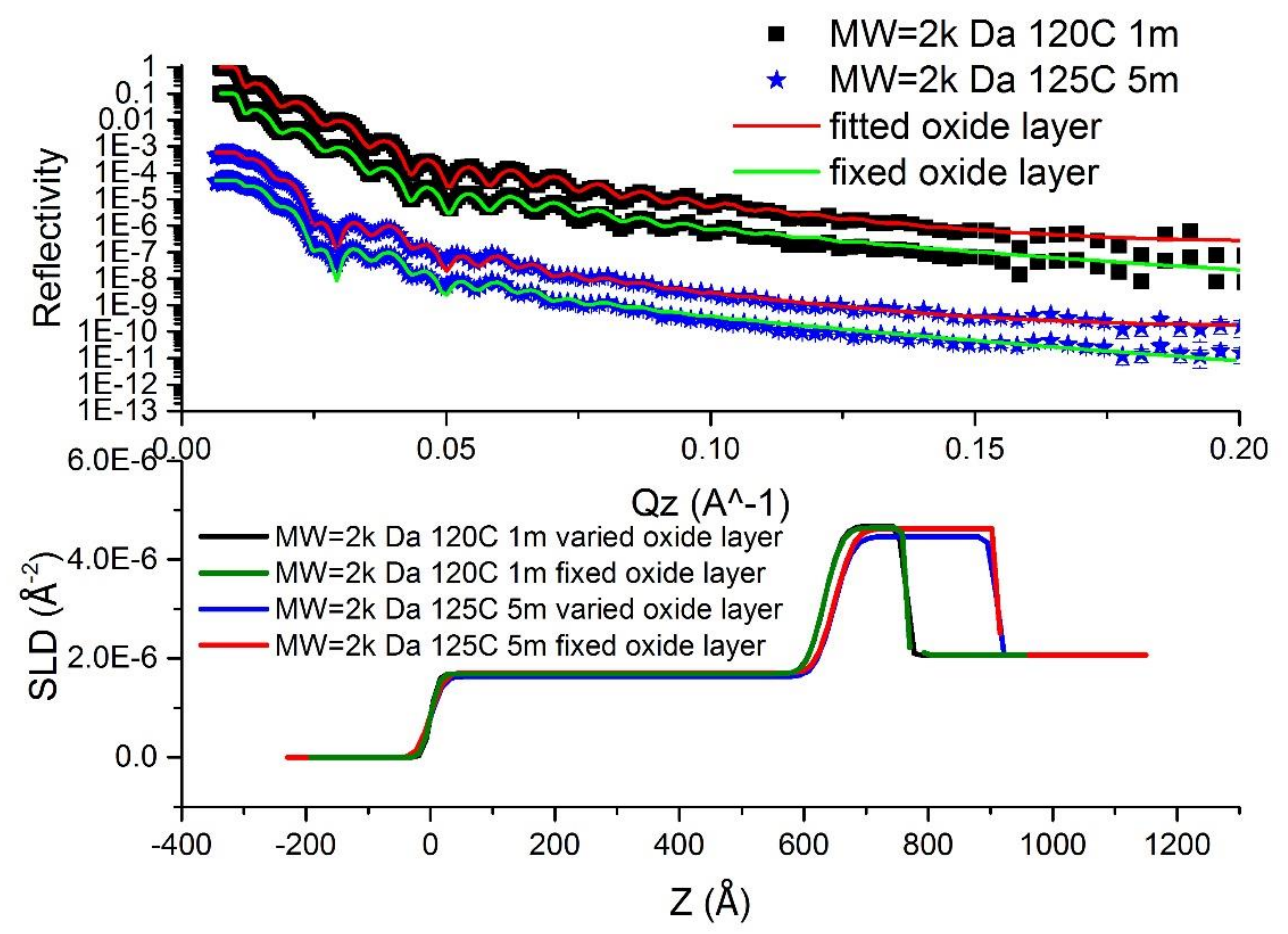

Figure 4.1-4: Two PCBM/ PS MW=2k Da samples with the oxide layer parameters allowed to vary, in comparison to fixed oxide layer fits. NR curves (top) and SLD profiles (bottom).The sample annealed shown that was annealed at $125^{\circ} \mathrm{C}$ is one of the double thickness samples discussed in section 4.1.7.

Many practical issues arose that could in-principle affect sample quality and therefore the reliability of extracted fit parameters. These were the appearance of inhomogeneities across the sample, splits in the top layer formed during floating, macroscopic variability due to dewetting, macroscopic thickness variation and variability in the oxide layer. There is also the potential for over-parametrisation of models used to fit the reflectivity curves. To reduce the potential for overparametrisation, the oxide layer values were fixed. All fits and SLD profiles shown in this thesis have fixed silicon oxide layer parameters (thickness, SLD and surface roughness), unless otherwise stated. As can be seen in Figure 4.1-4, allowing the oxide layer parameters to vary, between samples, enhances the fits very slightly. This 
is compared to the fixed oxide layer fits where there is a slight discrepancy between the data points and the fit between 0.1 to $0.15 \AA^{-1}$ (the SLD profile of these samples shows only a small difference between the SLDs, thickness and roughness of the two PCBM and PS rich layers). More information about the change in fit parameters after fixing the oxide layers can be found in the appendix. Overall the values for the interfacial roughness and the top layer SLD remain robust within error when the oxide layer is allowed to vary. In Figure 4.1-4 the samples with fixed oxide layers had chi-squared values of 4.8 and 7 for the $2 \mathrm{k} 120^{\circ} \mathrm{C} 1$ minute and $2 \mathrm{k} 125^{\circ} \mathrm{C} 5$ minute samples respectively, compared to chi-squared values of 4.7 and 5.9 for variable oxide layers. To determine the values for the fixed silicon oxide layer parameters, the oxide layers were initially fitted to each individual sample and then the mean of this was taken for each batch of silicon wafers, by experiment. More information about the effect of fixing the oxide layers (on a range of samples), as well as information regarding acceptable/unacceptable levels of inhomogeneities across samples, and why some sample fits were rejected, is given in the appendix.

As all roughnesses within a sample have the same qualitative effect of damping the fringes in the reflectivity, we have examined the potential correlation of the fitted roughnesses at both the surface and the interface during the fitting procedure. The effect of both roughnesses on the reflectivity curves and chi-squared contour maps are shown in the appendix to this chapter.

\subsubsection{The influence of annealing time}
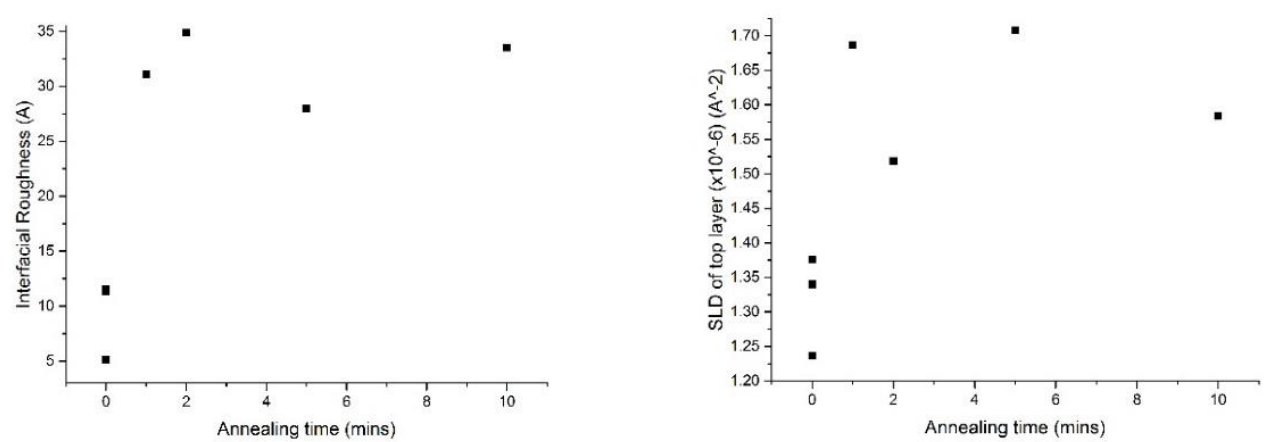

Figure 4.1-5: Interfacial roughness (left) and SLD of top layer (right) for bilayer samples of $P C B M / P S$ with $M W=2 k$ ex=situ annealed at various times at $120^{\circ} \mathrm{C}$ 
Figure 4.1-5 shows the interfacial roughness (left) and top layer SLD (right) as a function of annealing time. This data is for PCBM/PS MW=2k Da samples annealed at $120^{\circ} \mathrm{C}$ and shows no systematic change in either parameter value after annealing times as short as one minute. This justifies our chosen annealing times. These times were chosen to be short enough to prevent the PCBM from crystallising, and also to minimise potential problems such as dewetting, but long enough to allow liquidliquid equilibrium to be reached. These graphs show the most sensitive of our bilayers with-respect to sample quality, the $2 \mathrm{k}$ samples, suggesting that in our more stable systems (higher MW PS, which were less prone to dewetting) annealing times of 5 minutes as standard would lead to a stable film morphology. This was checked for higher MW PS, and it was found that equilibration occurred within one minute for these samples also. These annealing times and temperatures are also justified by in-situ measurements.

\subsubsection{Ex-situ annealing of PS/PCBM bilayers 3.5k and 5k PS}

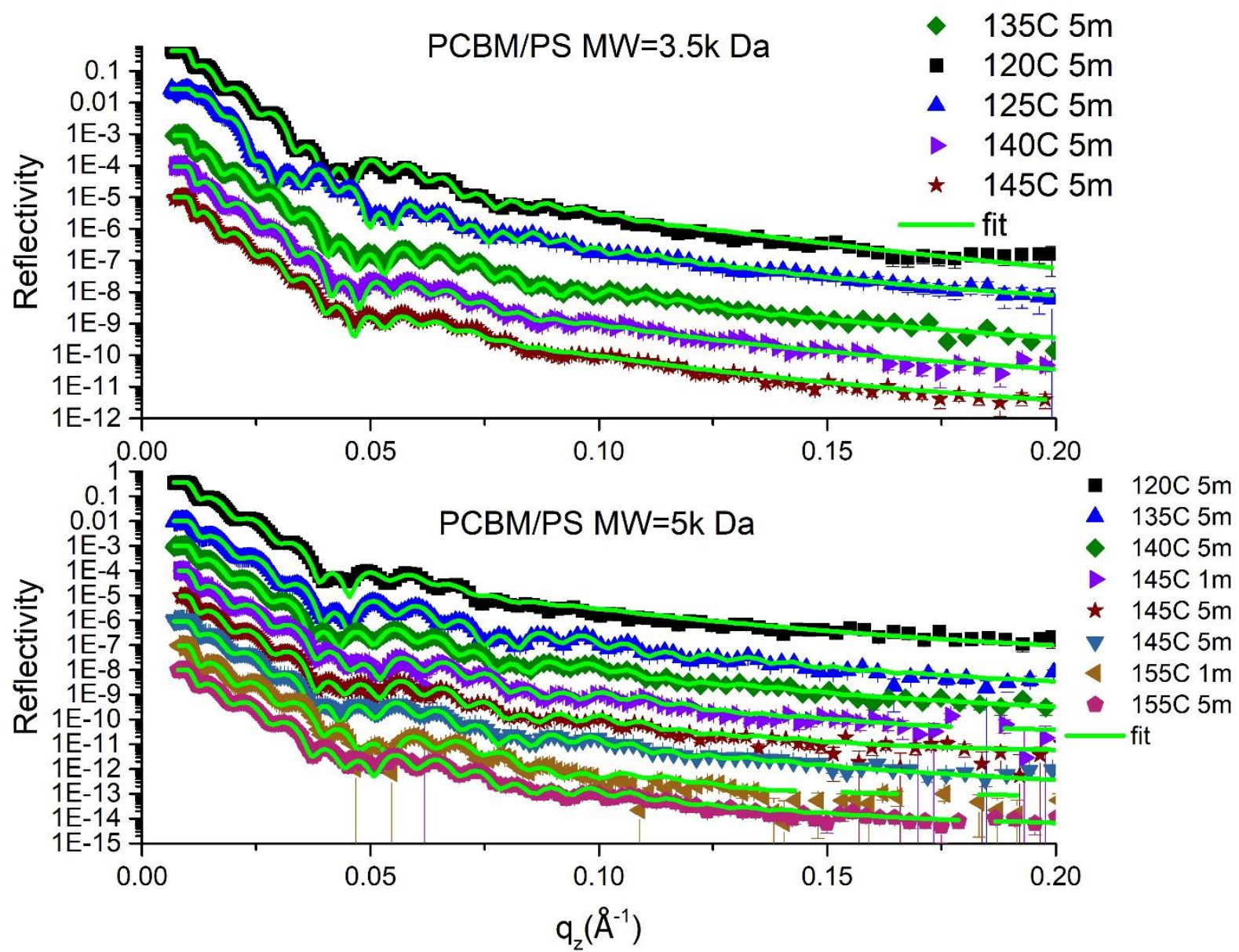

Figure 4.1-6: NR curves for PCBM/PS bilayers with PS MW=3.5k Da (top) and 5k Da (bottom) annealed at various temperatures. 


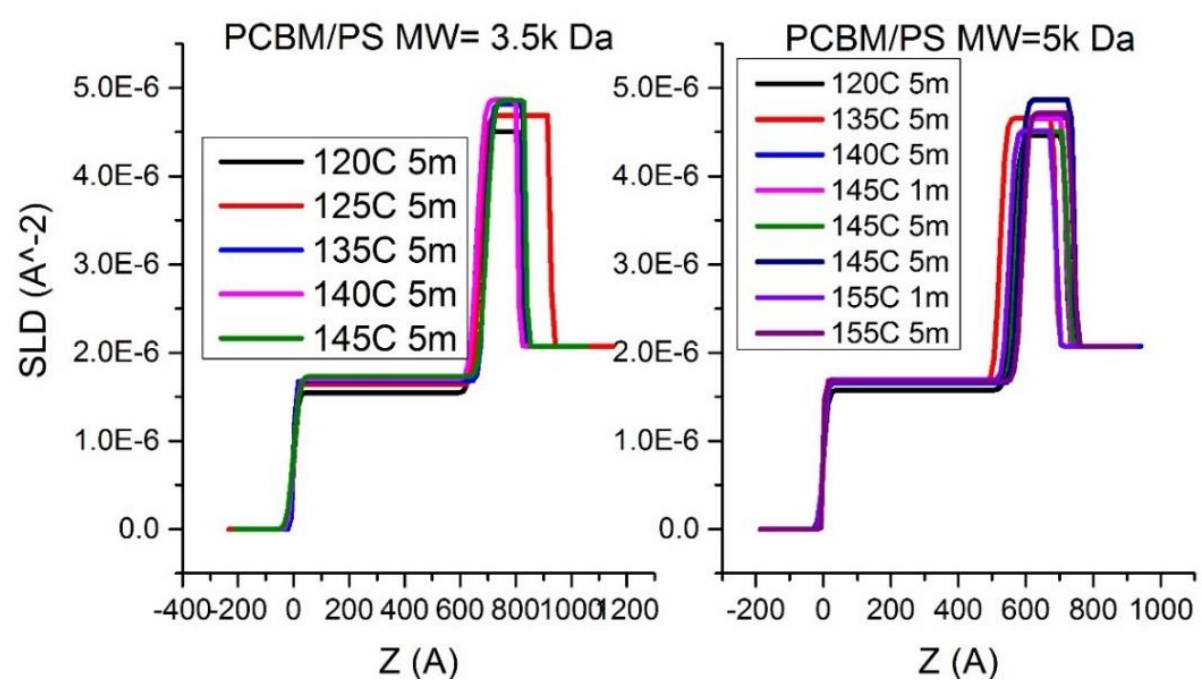

Figure 4.1-7: SLD profiles for PCBM/PS bilayers with PS MW=3.5k Da (left)and $5 k$ Da (right)annealed at various temperatures.

In Figure 4.1-6 and Figure 4.1-7 we see the same qualitative behaviour for the $\mathrm{PCBM} / \mathrm{PS} \mathrm{MW}=3.5 \mathrm{k}$ Da and $\mathrm{MW}=5 \mathrm{k}$ Da bilayers, as seen for the $2 \mathrm{k}$ bilayers: thinning of the bottom layer, an increase in the SLD of the top layer and a broadening of the interface. It is worth noting that four of the PS MW=5k Da curves have been annealed under the same conditions: two of these are duplicate samples and two are the same sample measured twice on different reflectometers. This will be discussed in section 4.1.6 as a means of assessing the different contributions to the experimental errors in the measurements.

\subsubsection{Ex-situ annealing of PS/PCBM bilayers PS 20k, 100k, 200k and 300k}

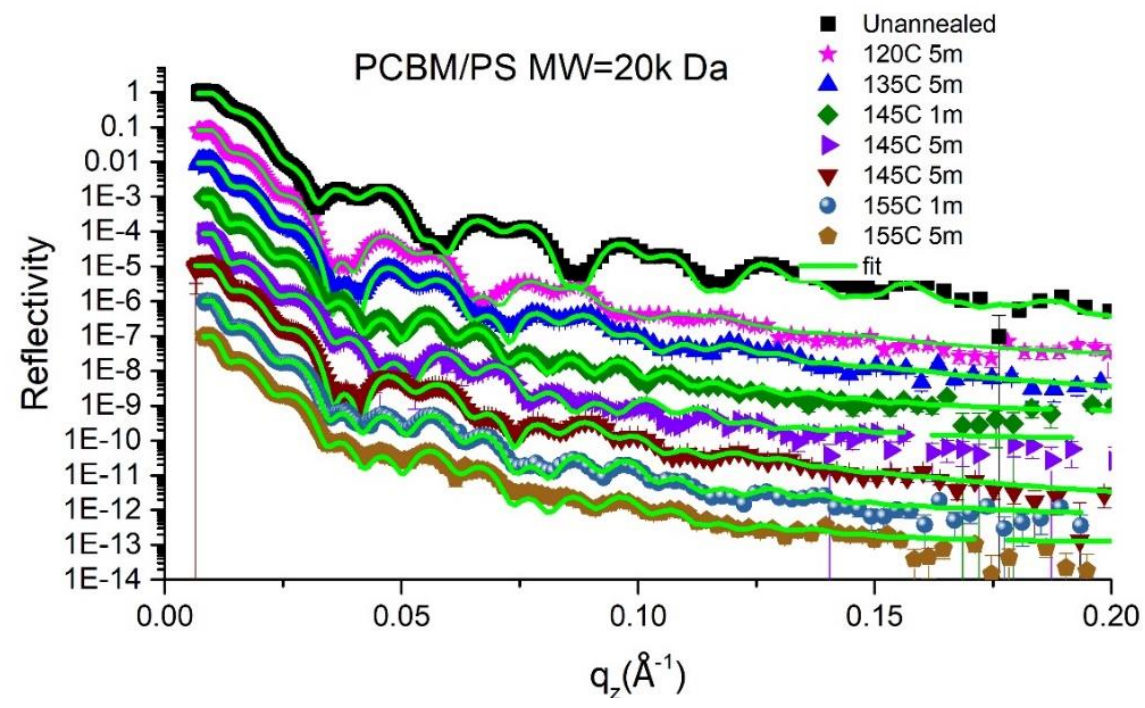

Figure 4.1-8: NR curves for PCBM/PS bilayers with PS MW=20k Da annealed at various temperatures. 


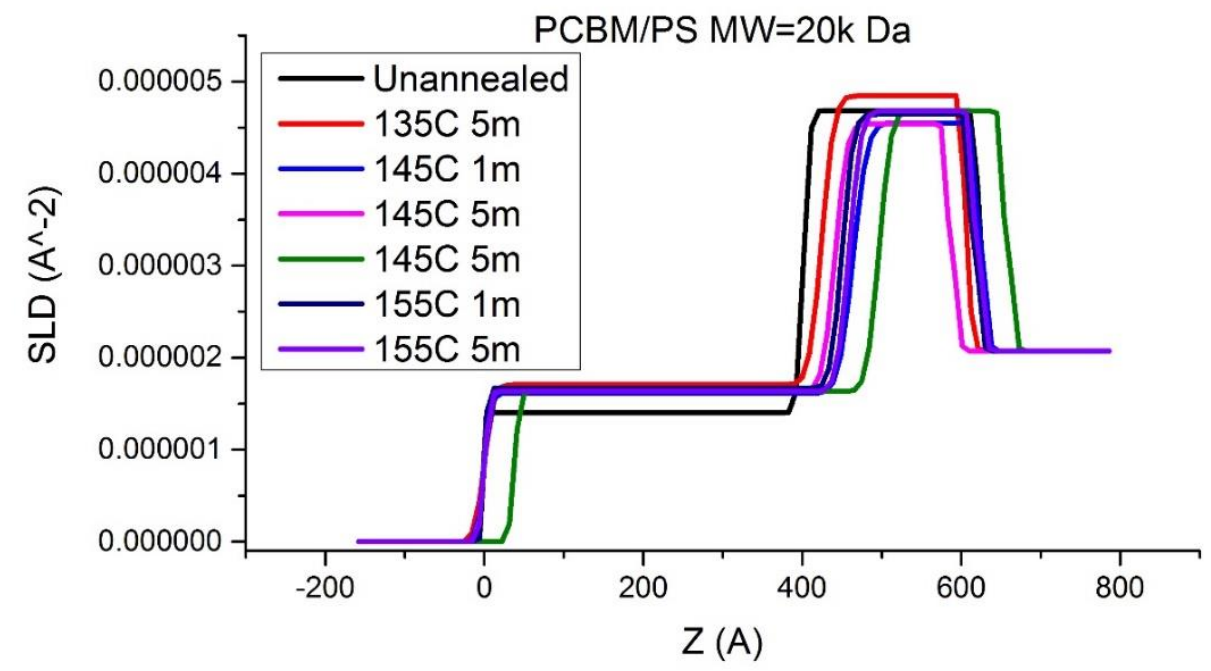

Figure 4.1-9: SLD profile for PCBM/PS bilayers with PS MW=20k Da annealed at various temperatures.

Figure 4.1-8 and Figure 4.1-9 show the NR curves and SLD profiles for PCBM/PS $\mathrm{MW}=20 \mathrm{k}$ Da samples including data from an unannealed sample. This shows an elevated top layer SLD, relative to the unannealed sample. The gradient of the interface in the SLD profiles is steeper than the MW=2k samples, indicating a sharper interface in terms of total roughness after annealing.

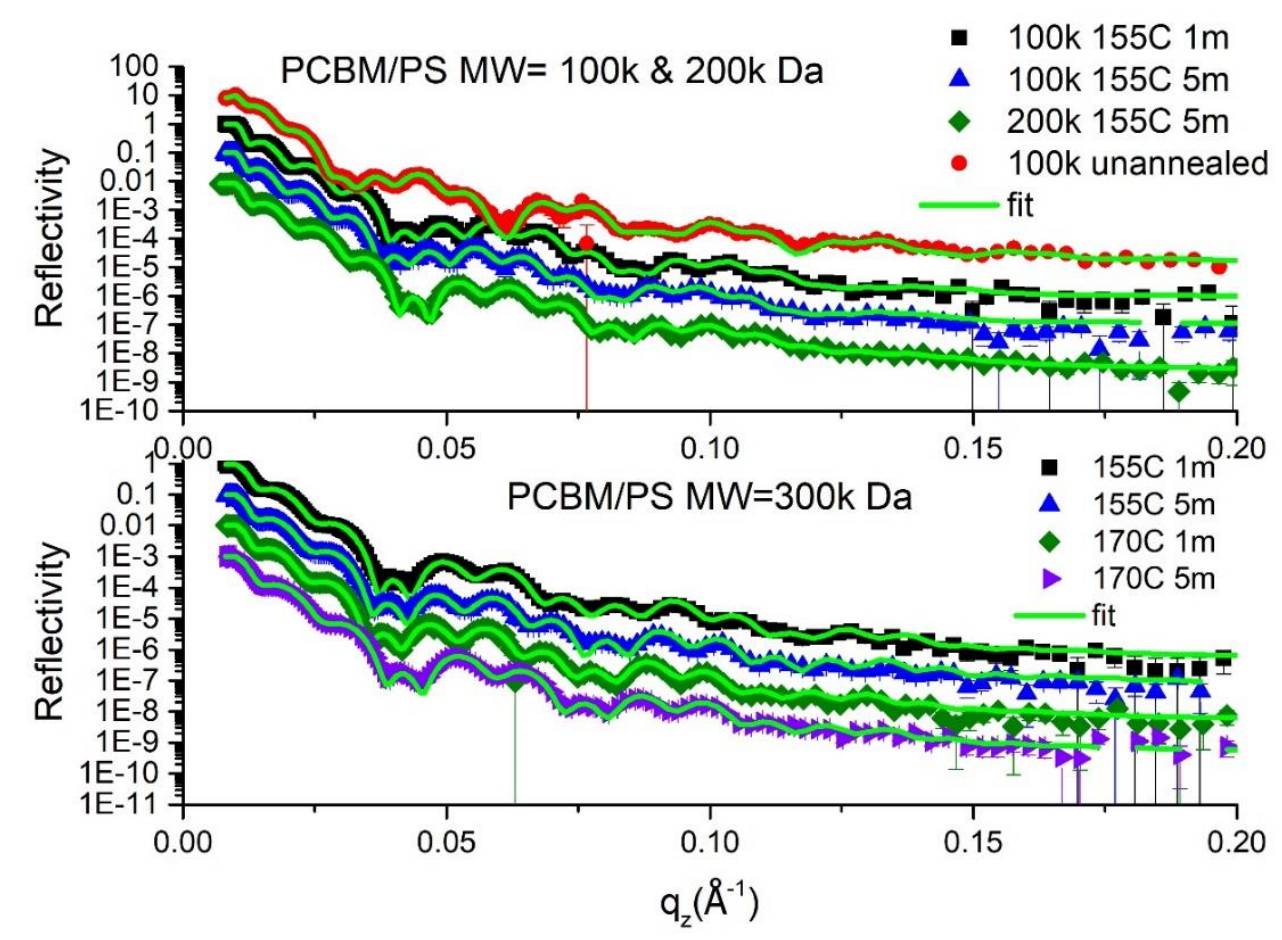

Figure 4.1-10: NR curves for PCBM/PS bilayers with PS MW=100k, 200k (top) and 300k (bottom) Da annealed at various temperatures. 


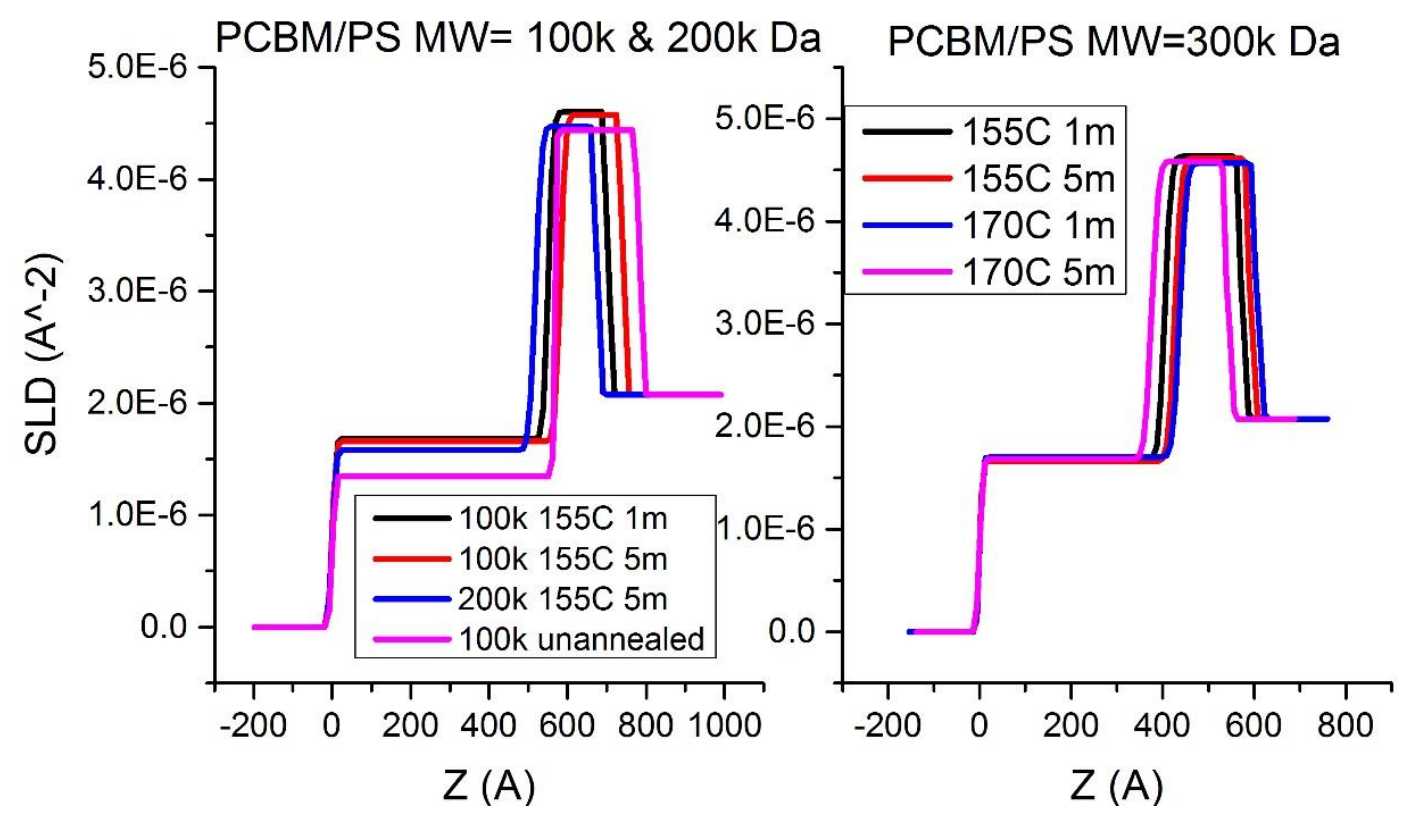

Figure 4.1-11: SLD profiles for PCBM/PS bilayers with PS MW=100k, 200k (left) and $300 k \mathrm{Da}$ (right) annealed at various temperatures..

In Figure 4.1-10 and Figure 4.1-11similar results were seen to what was seen for the lower molecular weights in terms of miscibility of the species, as indicated by the SLD of the top and bottom layers after annealing. However, there is a sharper interfacial roughness between the PCBM and the PS than for the lower MW.

\subsubsection{The impact of sample-to-sample variation and choice of reflectometer on reflectivity curves and fit parameters}

To assess the reproducibility of the results, three comparisons were performed. Figure 4.1-12 a) and b) and Figure 4.1-13 a) and b) show data from two pairs of samples with the same nominal sample parameters within each pair (same layer thicknesses and PS MW). The two pairs had PS MWs of 5k and 20k, and all four samples were annealed at $145{ }^{\circ} \mathrm{C}$ for 5 minutes. Comparing the fit parameters of these samples shows the typical level of reproducibility of thicknesses and the resulting fit parameters, using our fabrication protocol. This accounts for the potential error in setting the spin speed and making solutions of a given concentration, both of which affect the measured film thickness. The fitted parameter values for these samples are given in Table 3. Figure 1-12 c) and 1-13 c) show data for the same sample measured at two different reflectometers: INTER and D17. The values for their fitted parameters are shown in Table 5. The differences between these values give the experimental error, in terms of the reliability of the fitted parameters that have come from the NR measurements. In total, there are three 
duplicated samples for 5kPS and 20kPS demonstrating the reproducibility of our data in using the same sample fabrication procedure and neutron scattering methodology. The uncertainties measured here for the fitted parameters are small in comparison to the spread of the fit parameters seen for both different annealing times and temperatures. They are also small with respect to the spread seen as a function of MW that are discussed in section 4.1.9.

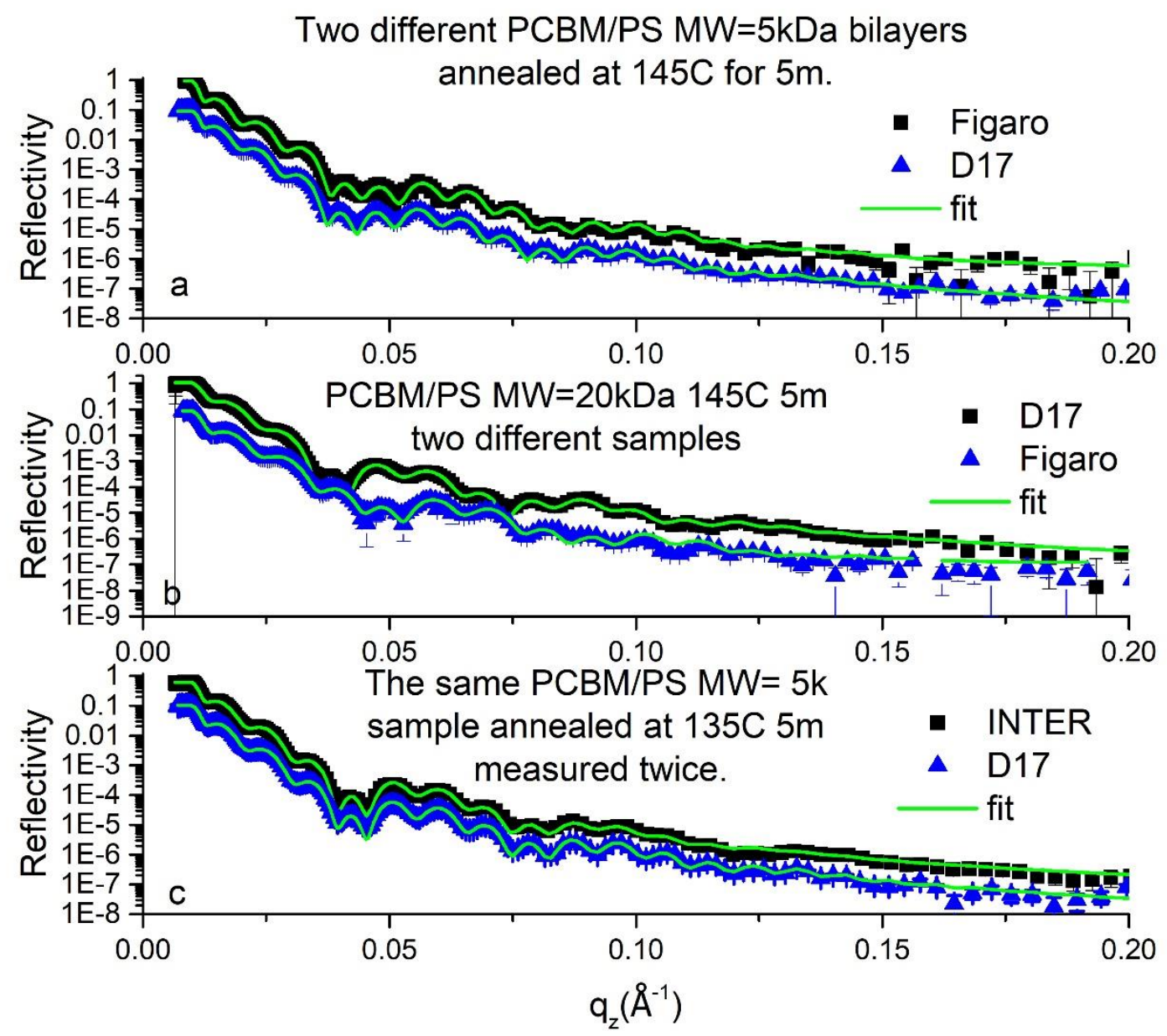

Figure 4.1-12:Data and fits for two different $M W=5 k$ Da bilayers annealed at the same temperature (a), two different $M W=20 k$ Da bilayers annealed at the same temperature (b) and the same sample measured twice (c) measured at different reflectometers; Figaro at the ILL, D17 at the ILL or INTER at ISIS. 


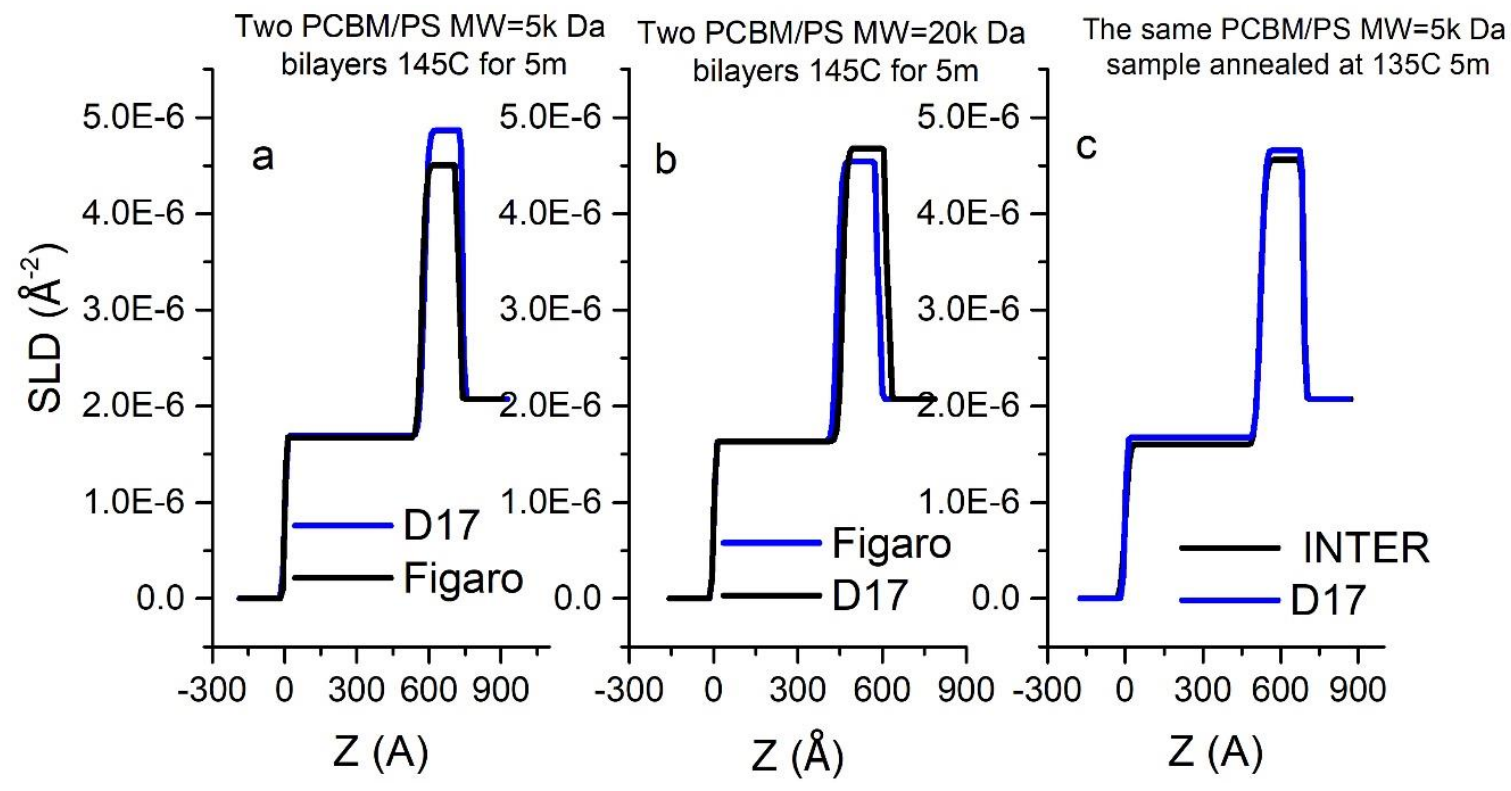

Figure 4.1-13: SLD profiles for data measured from the reproduced and repeated samples measured at different reflectometers.

\begin{tabular}{|l|l|l|l|l|l|l|}
\hline Instrument & $\begin{array}{l}\text { Thickness } \\
\text { of top lay- } \\
\text { er }(\AA)\end{array}$ & $\begin{array}{l}\text { SLD of top } \\
\text { layer } \\
(\times \\
\left.10^{-6} \AA^{-2}\right)\end{array}$ & $\begin{array}{l}\text { Roughness } \\
\text { of top lay- } \\
\text { er }(\AA)\end{array}$ & $\begin{array}{l}\text { Thickness } \\
\text { of bottom } \\
\text { layer }(\AA)\end{array}$ & $\begin{array}{l}\text { SLD of } \\
\text { bottom } \\
\text { layer } \\
(\times \\
\left.10^{-6} \AA^{-2}\right)\end{array}$ & $\begin{array}{l}\text { Roughness } \\
\text { of bottom } \\
\text { layer }(\AA)\end{array}$ \\
\hline D17 & 579.68 & 1.6921 & 10.134 & 157.09 & 4.8673 & 19.614 \\
\hline Figaro & 570.91 & 1.673 & 6.379 & 147.52 & 4.503 & 18.938 \\
\hline Variation & 8.77 & 0.0191 & 3.755 & 9.57 & 0.3643 & 0.676 \\
\hline
\end{tabular}

Table 3:fit parameters for two PCBM/PS $M W=5 \mathrm{k}$ bilayers made and annealed under the same protocol $(145 \mathrm{C} 5 \mathrm{~m})$ and measured at the Figaro reflectometer at the ILL and the D17 reflectometer at ISIS.

The duplicated Mw 5k samples also show variation from sample to sample and instrument to instrument within error. The reproducibility is robust and the difference is the same magnitude as our experimental error.

\begin{tabular}{|l|l|l|l|l|l|l|}
\hline Instrument & $\begin{array}{l}\text { Thickness } \\
\text { of top lay- } \\
\text { er }(\AA)\end{array}$ & $\begin{array}{l}\text { SLD of top } \\
\text { layer } \\
(\times \\
\left.10^{-6} \AA^{-2}\right)\end{array}$ & $\begin{array}{l}\text { Roughness } \\
\text { of top lay- } \\
\text { er }(\AA)\end{array}$ & $\begin{array}{l}\text { Thickness } \\
\text { of bottom } \\
\text { layer }(\AA)\end{array}$ & $\begin{array}{l}\text { SLD of } \\
\text { bottom } \\
\text { layer } \\
(\times \\
\left.10^{-6} \AA^{-2}\right)\end{array}$ & $\begin{array}{l}\text { Roughness } \\
\text { of bottom } \\
\text { layer }(\AA)\end{array}$ \\
\hline D17 & 428.7 & 1.5705 & 14.278 & 171.88 & 4.6522 & 19.541 \\
\hline Figaro & 440.89 & 1.639 & 7.815 & 140.54 & 4.544 & 17.07 \\
\hline Variation & 12.9 & 0.0685 & 6.463 & 31.34 & 0.1082 & 2.471 \\
\hline
\end{tabular}

Table 4: The fitted parameter values for two separate samples measured at two separate detectors but made and annealed with the same protocol; PCBM/PS $M W=20 k$ Da $145 C 5 m$. 


\begin{tabular}{|l|l|l|l|l|l|l|}
\hline Instrument & $\begin{array}{l}\text { Thick- } \\
\text { ness of } \\
\text { top layer } \\
(\AA)\end{array}$ & $\begin{array}{l}\text { SLD of } \\
\text { top layer } \\
(\times \\
10^{-6} \AA^{-2} \\
)\end{array}$ & $\begin{array}{l}\text { Rough- } \\
\text { ness of } \\
\text { top layer } \\
(\AA)\end{array}$ & $\begin{array}{l}\text { Thick- } \\
\text { ness of } \\
\text { bottom } \\
\text { layer }(\AA)\end{array}$ & $\begin{array}{l}\text { SLD of } \\
\text { bottom } \\
\text { layer } \\
(\times \\
10^{-6} \AA^{-2} \\
)\end{array}$ & $\begin{array}{l}\text { Rough- } \\
\text { ness of } \\
\text { bottom } \\
\text { layer }(\AA)\end{array}$ \\
\hline INTER & 521.8 & 1.596 & 17.455 & 161.84 & 4.5626 & 21.192 \\
\hline D17 & 523.6 & 1.6742 & 8.6851 & 161.81 & 4.655 & 18.652 \\
\hline $\begin{array}{l}\text { Experi- } \\
\text { mental Error }\end{array}$ & 1.8 & 0.0782 & 8.7699 & 0.03 & 0.0924 & 2.54 \\
\hline
\end{tabular}

Table 5: The six fit parameter values for the same PCBM/PS MW=5k Da sample (annealed at $135{ }^{\circ} \mathrm{C}$ for 5 minutes) measured twice, once at INTER detector at ISIS and once at the D17 detector at the ILL.

\subsubsection{Film thickness considerations}

Layer thickness could, in principle, influence the measured composition profile in PCBM/PS bilayers. Theoretical predictions for lateral roughness due to thermal capillary-waves at an equilibrium liquid-liquid interface or liquid surface, state that this will in principle have a dependence on the thicknesses of the liquid films involved, due to the influence of van der Waals forces across the thin-films on the capillary-wave spectrum (see equation 2.53) (Sferrazza et al. 1997). In PCBM/PS it was not expected that lateral roughness changes with layer thickness would be particularly significant, due to the absence of visible Yoneda scattering.

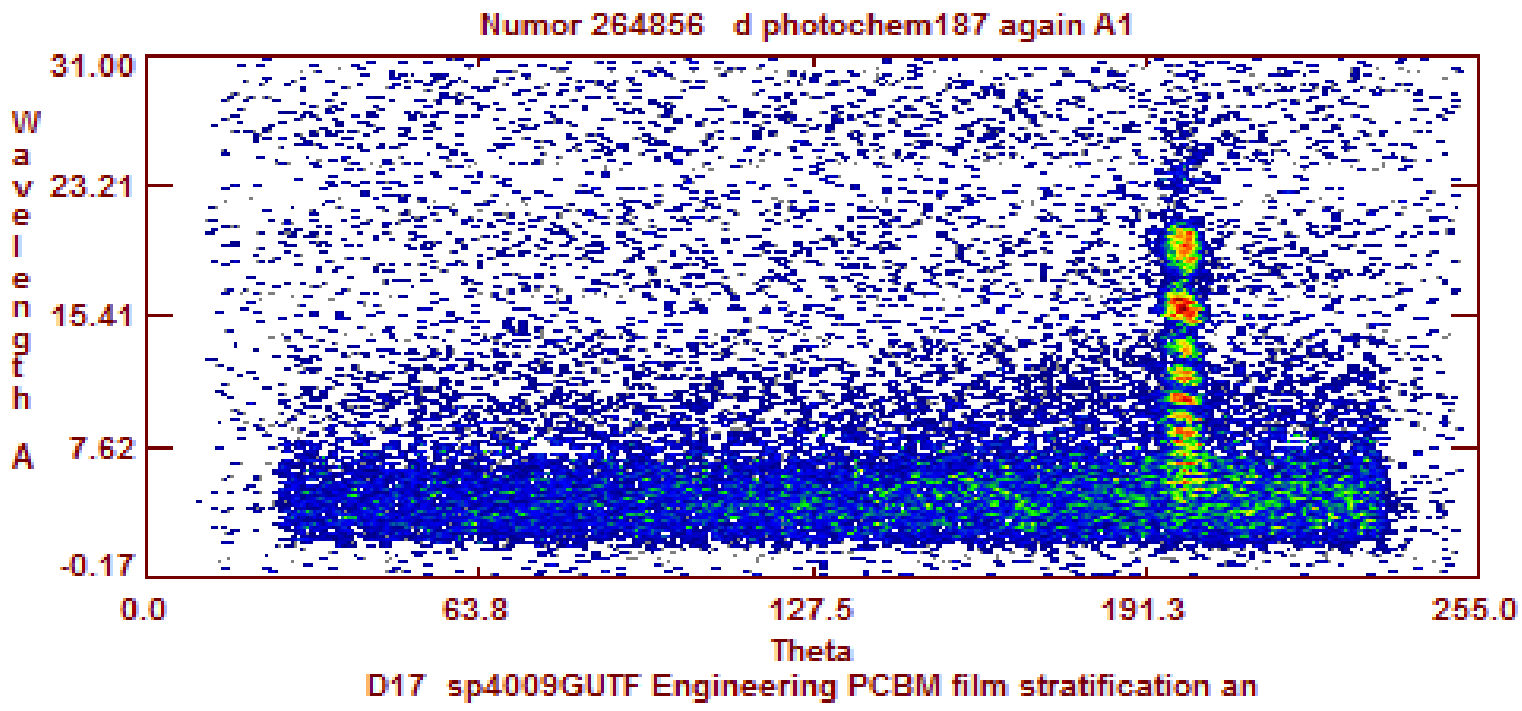

Figure 4.1-14: Detector map of a sample showing no visible Yoneda peak.

Figure 4.1-14 shows a detector map showing the strong specular reflection but no discernible off-specular reflection, such as would be seen from a Yoneda peak. A 
Yoneda peak would tell us about the thermal capillary roughness (lateral roughness) as separate from the combined thermal and molecular roughness that is measured from the specular region, and there is no significant peak in our data (for counting times on the order of one hour). This indicates that there is no significant lateral roughness (on lateral length-scales that are accessible in the detector map- typically a few hundred $\mathrm{nm}$ to several microns) to take into account and, on this assumption, the total interfacial roughness measured can be compared to predictions for intrinsic roughness for polymer-polymer mixtures. However, in addition to probing the thickness-dependence of lateral roughness, it was also necessary to ensure that the layers are thick enough that the width of the buried interface remains significantly smaller than the thickness of the layers. Failure to do this could potentially invalidate the fitting of the composition profile using an error function (or a tanh function, see equation 2.46). Mon et al (Môn et al. 2015) examined the influence of PS film thickness in PCBM/PS bilayers on composition profiles and on PCBM crystallisation. They found that the thickness of the PS layer did not significantly affect the SLD profiles; in terms of either the layer compositions or the interfacial roughness. However, as stated in chapter 1.2, Mon et al found that PCBM crystallisation behaviour was PS-thickness-dependent. This included the growth of nanocrystals and also micron-sized crystals that could disrupt the bilayer architectures. To determine the presence or absence of significant crystallisation occurring within (shorter) annealing times (which was showed to be sufficient for equilibrium) GIXD was performed on a selection of PCBM/PS bilayers. Only an amorphous PCBM ring was visible in all samples measured, with no PCBM Bragg peaks, indicating no significant crystallisation of the PCBM (see the appendix to this chapter for further details). To avoid any potential PS thickness-dependent behaviour (such as depressed glass transition temperature as discussed in section 2.3), the PS layers in the present study were all of $40 \mathrm{~nm}$ or above.

Mon et al did not perform any reflectivity measurements as a function of PCBM film thickness (all unannealed PCBM layers were 20nm thick). In the present study, the sensitivity of the composition profiles to the thickness of the PCBM layer was probed by making several 2k PS bilayers with different bottom layer thicknesses. As stated in section 4.1.1, the majority of the PCBM/PS bilayers had an initial (unannealed) PCBM layer thickness of approximately 21nm. Figure 4.1-15 and 


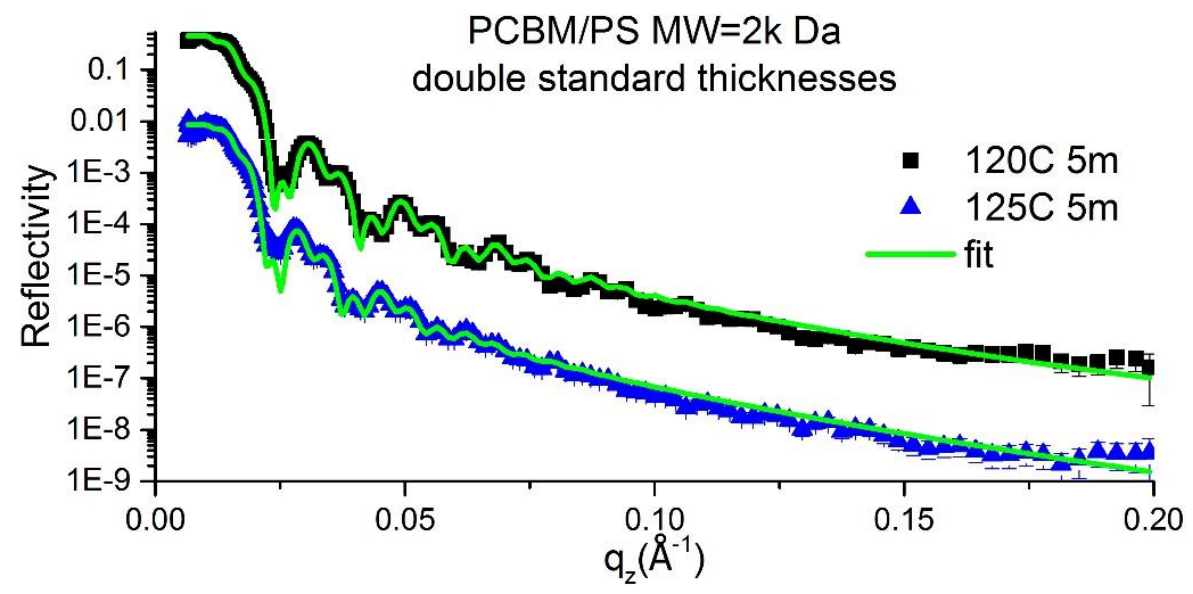

Figure 4.1-15: NR curves and fits for PCBM/PS bilayers with PS $M W=2 k$ Da and the thickness of each layer double the standard thickness.

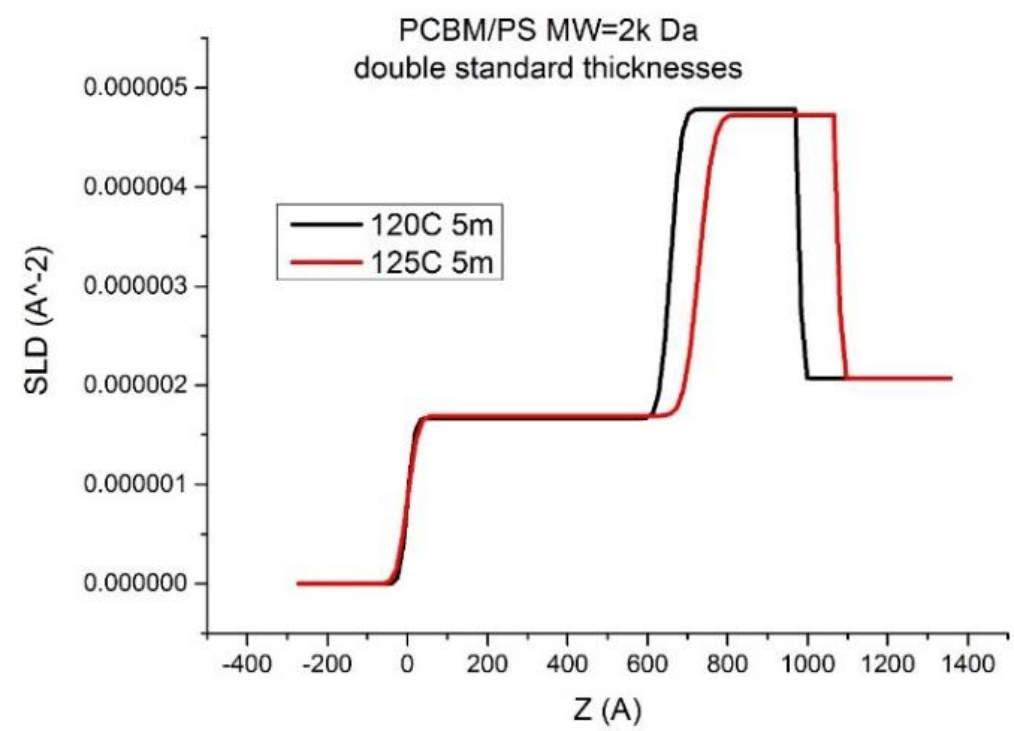

Figure 4.1-16: SLD profiles for PCBM/PS bilayers with PS MW=2k Da and the thickness of each layer thicker than the standard thickness.

Figure 4.1-16 show measurements of two further samples with thicker PCBM layers (the unannealed samples had $35 \mathrm{~nm}$ PCBM layers). Figure 4.1-16 shows the composition profiles for the two samples with the thickest bottom (PCBM) layers. The reflectivity curves for these samples are shown in Figure 4.1-15. The NR curves from these thicker samples have shorter period fringes in comparison to Figure 4.1-2, but little difference is seen in the top and bottom layer SLDs and the interfacial roughness (see Table 6 ). One thing that is important to note here is that the $125^{\circ} \mathrm{C}$ sample in Figure 4.1-15 had a significant coverage by macroscopic defects (see the appendix to this chapter). For this reason, the fit parameters from this sample are not 
included in the final analysis in section 4.1.9 below. However, it is interesting to point out that this macroscopic inhomogeneity across this sample, had surprisingly little impact on the fitted SLD profile shown in Figure 4.1-15 (in comparison to the $120{ }^{\circ} \mathrm{C}$ sample, in which there was excellent uniformity across the sample). The top layer SLDs of the two samples in Figure 4.1-15 are very similar, and the only significant difference is the higher interfacial roughness of the $125^{\circ} \mathrm{C}$ sample $(40.5$ $\AA$ in comparison to $29 \AA$ for the $120{ }^{\circ} \mathrm{C}$ sample).

\begin{tabular}{|l|l|l|l|l|l|l|}
\hline Sample & $\begin{array}{l}\text { Thickness } \\
\text { of top } \\
\text { layer }(\AA)\end{array}$ & $\begin{array}{l}\text { SLD of } \\
\text { top layer } \\
(\times \\
\left.10^{-6} \AA^{-2}\right)\end{array}$ & $\begin{array}{l}\text { Surface } \\
\text { roughness } \\
(\AA)\end{array}$ & $\begin{array}{l}\text { Thickness } \\
\text { of bottom } \\
\text { layer }(\AA)\end{array}$ & $\begin{array}{l}\text { SLD of } \\
\text { bottom } \\
\text { layer } \\
(\times \\
\left.10^{-6} \AA^{-2}\right)\end{array}$ & $\begin{array}{l}\text { Interfacial } \\
\text { roughness } \\
(\AA)\end{array}$ \\
\hline 2k 120C & 663 & 1.7 & 17.8 & 241 & 4.69 & 27.9 \\
\hline 2k 120C & 657 & 1.67 & 19.4 & 319 & 4.78 & 29.4 \\
\hline 2k 125C & 650 & 1.7 & 22.8 & 258 & 4.62 & 33.6 \\
\hline
\end{tabular}

Table 6:Comparison of parameters for different thicknesses (all annealed for 5mins). These are the thickest samples shown in Figure 4.1-17 and Figure 4.1-18 below.

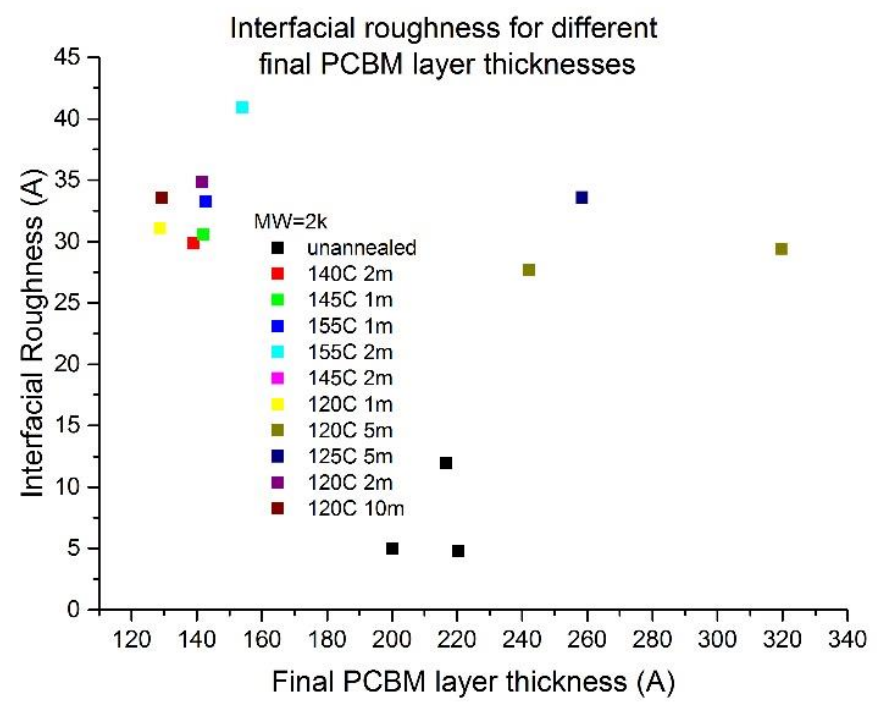

Figure 4.1-17:Interfacial roughness post-annealing for a range of post-annealing thicknesses of the bottom layer. 


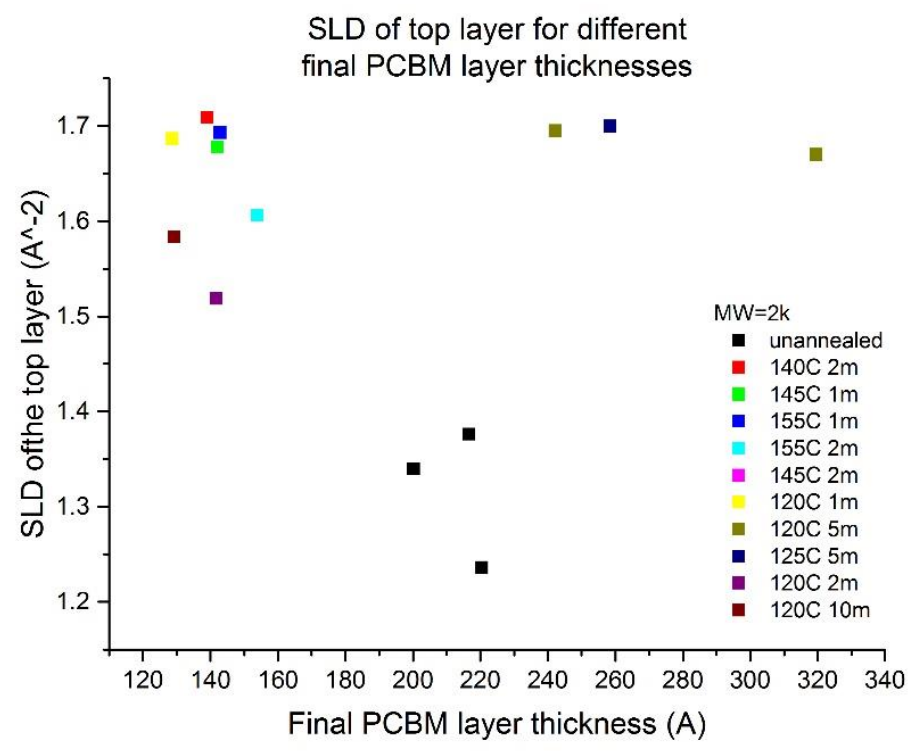

Figure 4.1-18: SLD of the top layer post-annealing for a range of post-annealing thicknesses of the bottom layer.

The full effect of PCBM layer thickness is quantified in Figure 4.1-17 and Figure 4.1-18. These show the interfacial roughness and top layer SLD as a function of the final thickness of the bottom layer, for all of the 2K PS samples (in comparison to the unannealed 2k PS samples). No systematic changes in either parameter are evident as a function of PCBM layer thickness. The variation in measured interface widths seen in Figure 4.1-17 is not significant compared to the differences between these samples and the higher MW. The implications of these ex-situ results are that, as a function of PCBM and PS thickness (studied by Mon et al), consistent layer compositions are formed. The implications from the ex-situ measurements, as a function of annealing time, show that consistent coexisting layer compositions are formed of around 10\% and 100\% PCBM. It is proposed that these two compositions are co-existing phases in a liquid-liquid system at thermodynamic equilibrium. This is discussed in more detail in Section 4.1.9 below with attention to the effect of MW on the composition profile. Before this is discussed, in-situ measurements are presented.

\subsubsection{In-situ annealing}

This section describes in-situ annealing measurements that were performed in the neutron beam on eight different samples. Four of these were duplicates of the ex-situ annealed PCBM/PS (with MW of 2k, 3.5k, 20k and 100k) bilayers discussed 
previously, and two were single layers (one PCBM and one PS). Two further samples, in which the top layer started off as a PS-rich blend with PCBM (on top of a pure PCBM layer), were also measured. In-situ NR experiments were performed on the PCBM/PS bilayers to corroborate the ex-situ measurements and to probe for temperature dependence in this system. This was done by annealing in steps below and above the bulk glass transition temperature of both materials until a clear change in the shape of the reflectivity profile was observed. Following these changes, which indicate mixing and interfacial broadening occurring, the temperature was then set to $30^{\circ} \mathrm{C}$ and the sample allowed to cool. This was to probe whether there was any effect of quenching used in the ex-situ sample preparation on the film composition compared to slow cooling from similar temperatures. There are no significant differences between the profiles obtained from slow cooling from high temperature (in-situ annealing) and rapid quenching on a metal block (ex-situ annealing).

The cooling rate was much slower than when quenching was applied to the ex-situ samples. For the $2 \mathrm{k}$ sample, the fit of a full NR curve following in-situ annealing doesn't reproduce the fringes well and significant lateral inhomogeneities are found on some (but not all) of these in-situ samples after annealing (see appendix at the end of this chapter). However, no evidence of significant crystal formation is seen from GIXD on these samples. The in-situ sample parameters following annealing are not included in our ex-situ comparison of SLDs and interface roughness in section 4.1.9.

These measurements were similar to the in-situ measurements performed by Mon et. al, although the MW in this set was lower (2k-20k, in comparison to $344 \mathrm{k}$ used by Mon et al), which posed challenges to avoid dewetting during annealing. Two actions were followed in fitting the kinetic data: summing together four 30s slices, and fitting individual 30s slices. The individual 30s slice data is fitted to look at the kinetics in time periods where the four summed slices indicate changes in the fitted parameter values. Adding together four 30s measurements reduces the error bars. However, as can be seen in the $20 \mathrm{k}$ sample, there is some potential overparametrisation of these kinetic slices. In the $20 \mathrm{k}$ sample we see a correlation between jumps in two parameter values. In general, the kinetic fits are reliable qualitatively, but are quantitatively not as accurate as the ex-situ and full NR curves measured before and after the in-situ heating. 

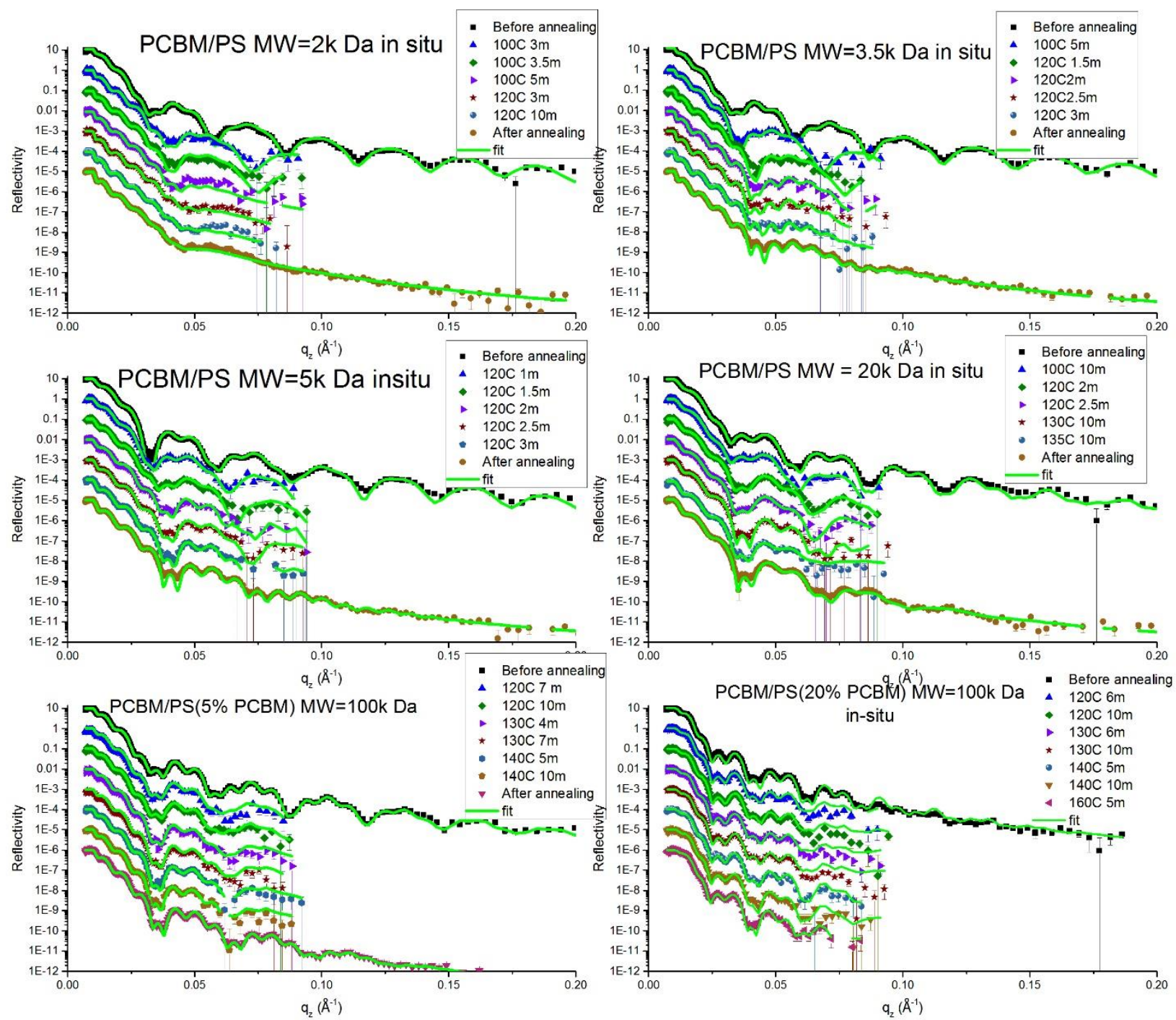

Figure 4.1-19: In-situ data for samples indicated in the headings. Full NR curves taken before and after annealing, shorter NR curves are 30s kinetic measurement showing distinctive visual changes in the shape of the curve at low $Q$ indicating a change in parameters. 

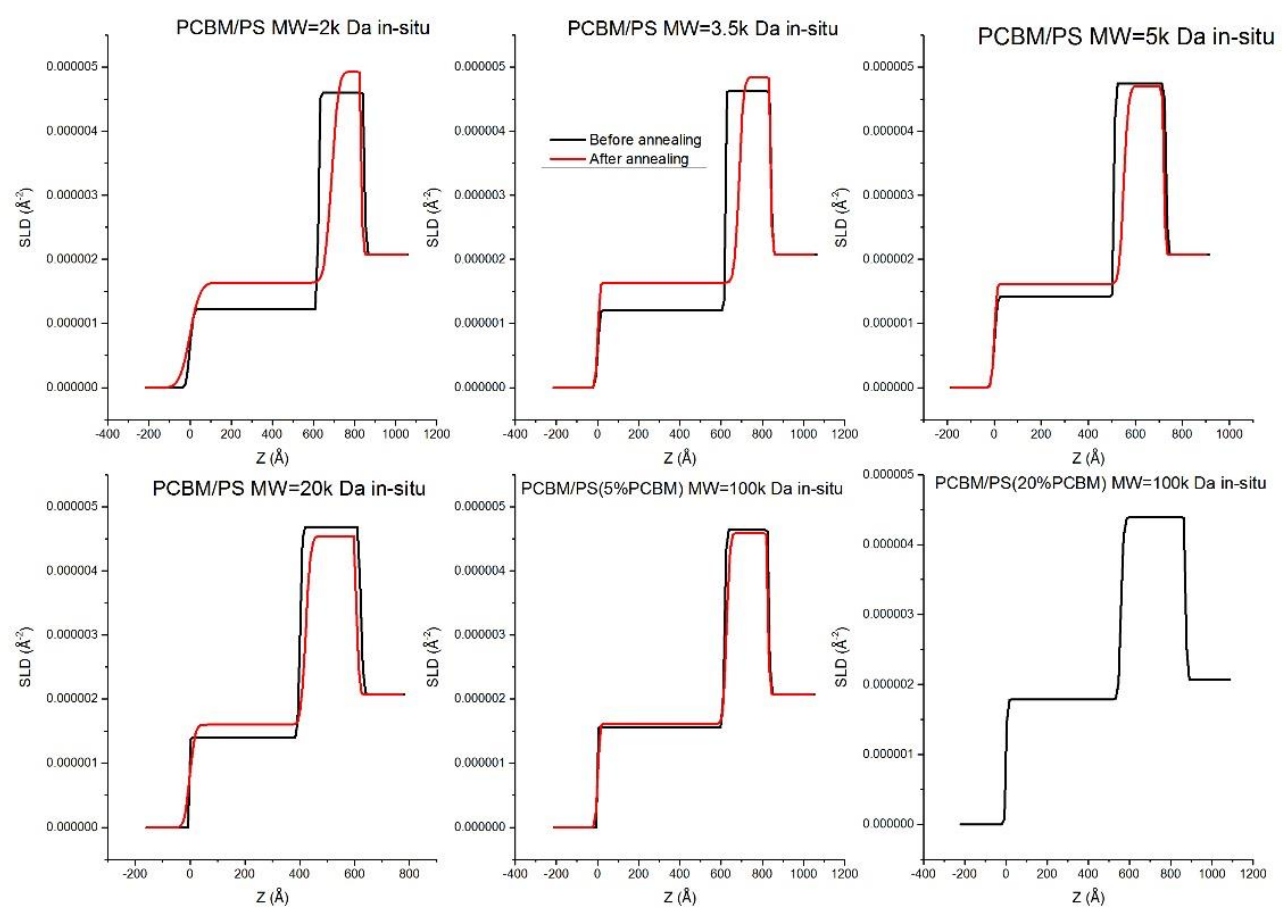

Figure 4.1-20: In-situ SLD profiles for the before and after annealing measurements for the samples indicated in the headings.

Figure 4.1-19 and Figure 4.1-20 show the in-situ NR curves and SLD profiles, for bilayers, taken before and after in-situ heating in the neutron beam. Figure 4.1-21 shows the NR curves for two single layer samples. The shorter NR curves that only extend to 0.1 in $\mathrm{Q}$ are the 30s kinetic measurements taken which show the progressive change in the measured curve, albeit with larger error bars due to the shorter counting times. These graphs also show a sample prepared with an initial top layer composition of 5\% by volume PCBM blended with the PS. This sample was prepared to probe the free energy landscape surrounding the observed (equilibrium coexisting) layer compositions found in the ex-situ samples annealed from two initially pure layers. In this case, the top layer SLD increased on annealing to the level seen for the initially pure phase samples. A similar blended top layer is shown but starting from a composition on the other side of the proposed free energy minimum, with a $20 \%$ by volume PCBM content in the PS-rich top layer before annealing. The kinetic measurements do indicate that the sample again approached the SLD values seen for annealed initially pure phases but this sample dewetted and 
became too rough to measure before the heating and cooling cycle was complete so we do not have a full reflectivity curve for after the annealing to compare it to. For the blended top layer samples there was a slower evolution rate towards equilibrium as one would expect from slower diffusion between two phases that are closer in composition to each other. In all of the in-situ samples qualitatively similar changes in SLD profile were seen to those observed in the ex-situ measurements. The compositions progressed from pure (or 5\% and 20\% PCBM) top layers with a sharp interface to (or towards) stable coexisting compositions of approximately $10 \%$ and $100 \%$ PCBM. The evolution of these samples' parameters with time is shown in Figure 4.1-22 to Figure 4.1-28.

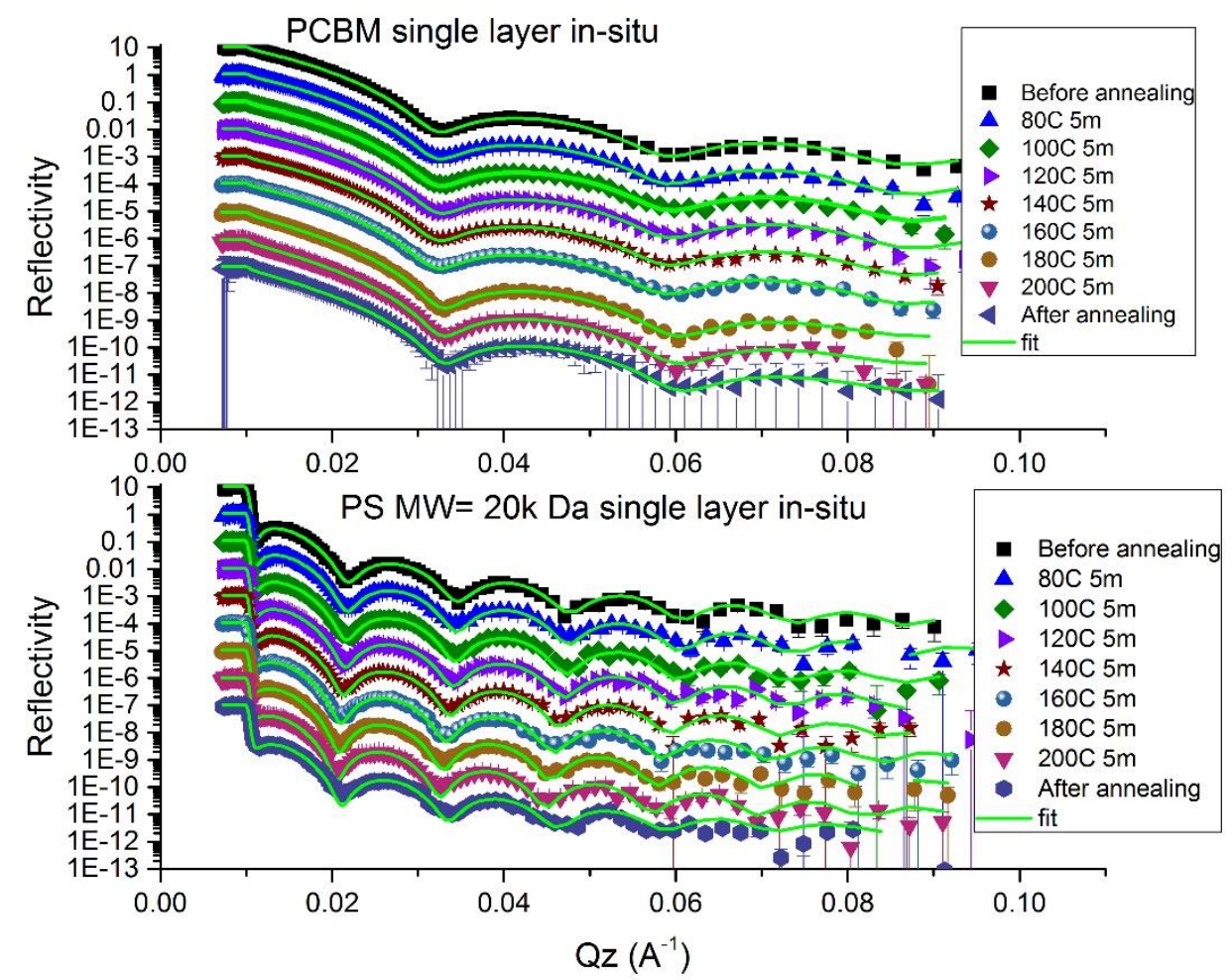

Figure 4.1-21: Kinetic measurements of the reflectivity curve of a PCBM single layer, and a PS MW=20k Da single layer. 


\section{$\mathrm{PCBM} / \mathrm{PS} \mathrm{MW}=2 \mathrm{k}$ Da in-situ fit parameters}
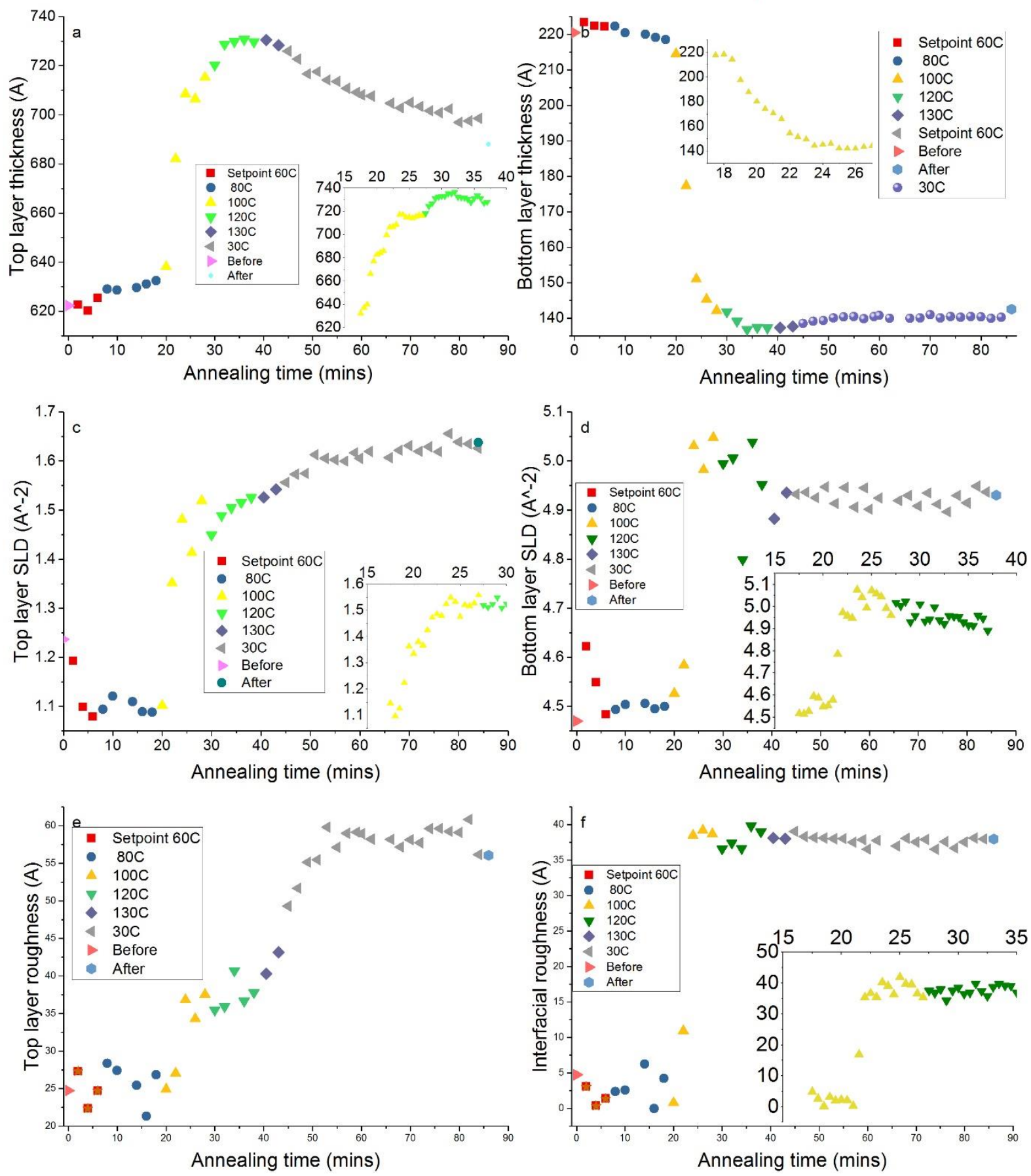

Figure 4.1-22:The change in fit parameters over time for PCBM/PS $M W=2 k D a$. Each data point is for reflectivity summed over four 30s measurements barring the inset graphs which show data points for individual 30s measurements. Graph a is thickness of the top layer (PS-rich), graph $b$ is the thickness of the bottom layer (PCBM), graph $c$ is the SLD of the top layer, $d$ is the SLD of the bottom layer, $e$ is the top layer roughness and $f$ is the interfacial roughness. 

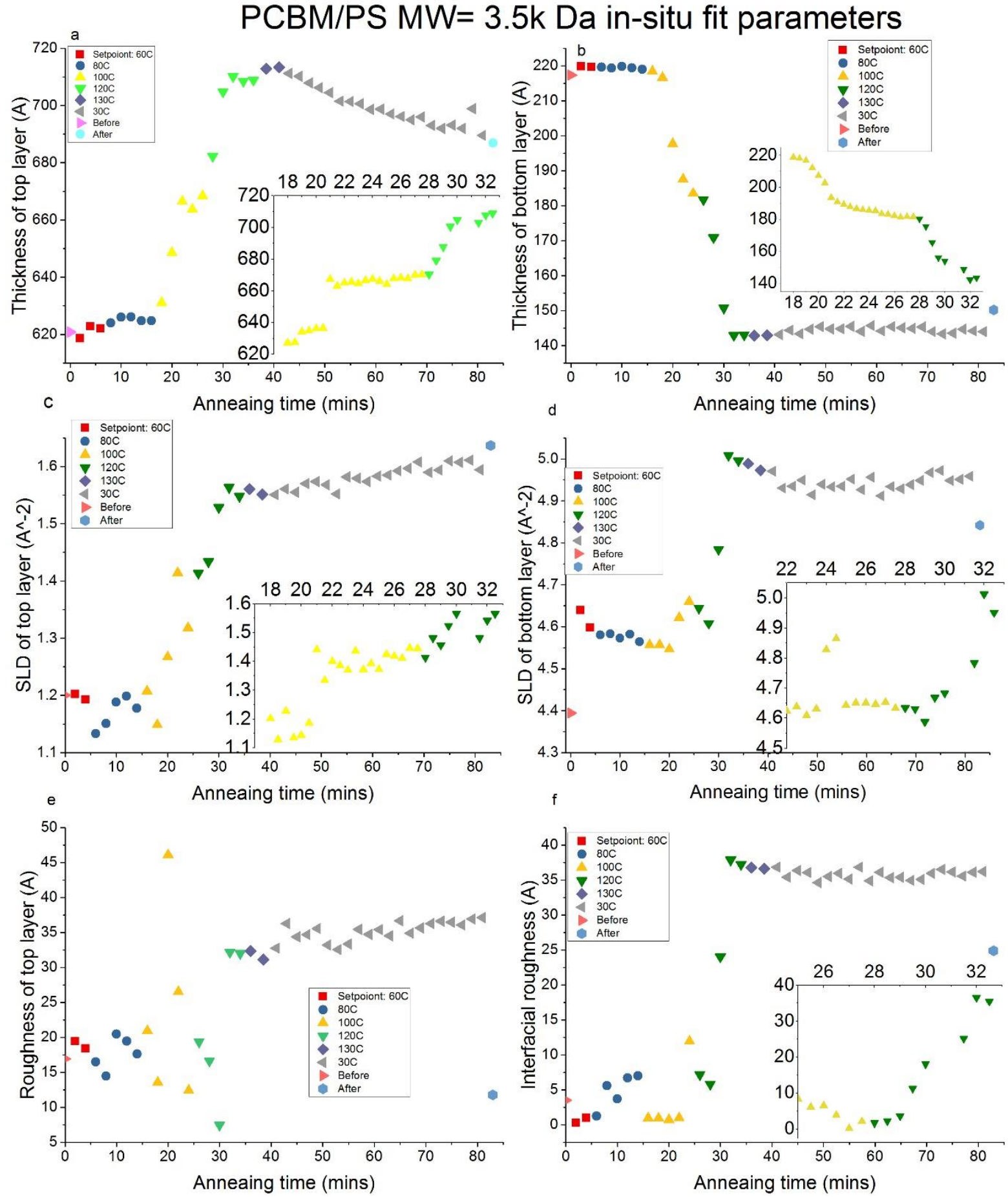

Figure 4.1-23: The change in fit parameters over time for PCBM/PS $M W=3.5 \mathrm{kDa}$.

Each data point is for reflectivity summed over four 30s measurements barring the inset graphs which show data points for individual 30s measurements. Graph a is thickness of the top layer (PS-rich), graph $b$ is the thickness of the bottom layer (PCBM), graph c is the SLD of the top layer, $d$ is the SLD of the bottom layer, $e$ is the top layer roughness and $f$ is the interfacial roughness. 


\section{$\mathrm{PCBM} / \mathrm{PS} \mathrm{MW}=5 \mathrm{k}$ Da in-situ fit parameters}
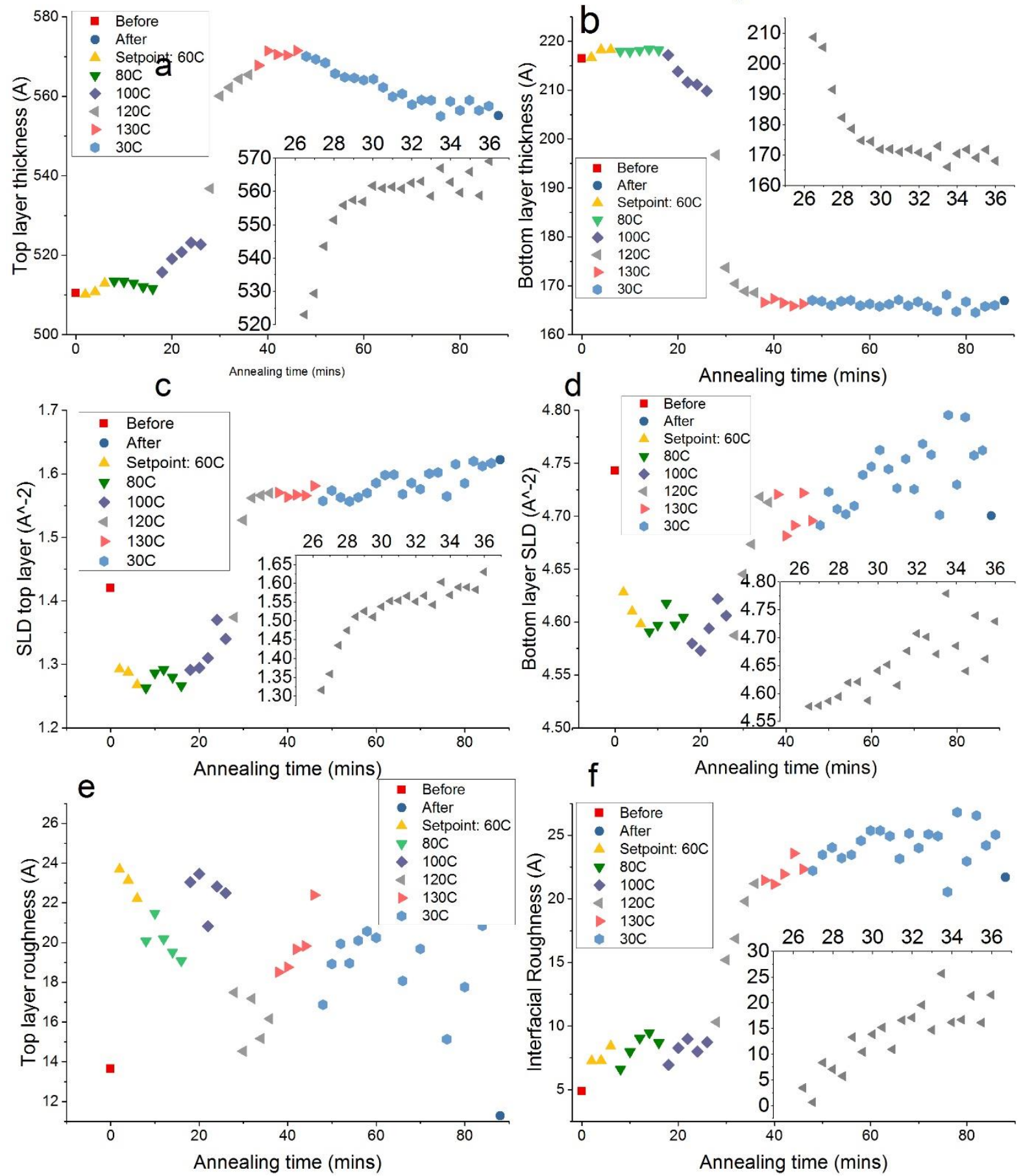

Figure 4.1-24: The change in fit parameters over time for PCBM/PS MW=5kDa.

Each data point is for reflectivity summed over four 30s measurements barring the inset graphs which show data points for individual 30s measurements. Graph a is thickness of the top layer (PS-rich), graph $b$ is the thickness of the bottom layer (PCBM), graph $c$ is the SLD of the top layer, $d$ is the SLD of the bottom layer, $e$ is the top layer roughness and $f$ is the interfacial roughness. 


\section{PCBM/PS MW= 20k Da in-situ fit parameters}
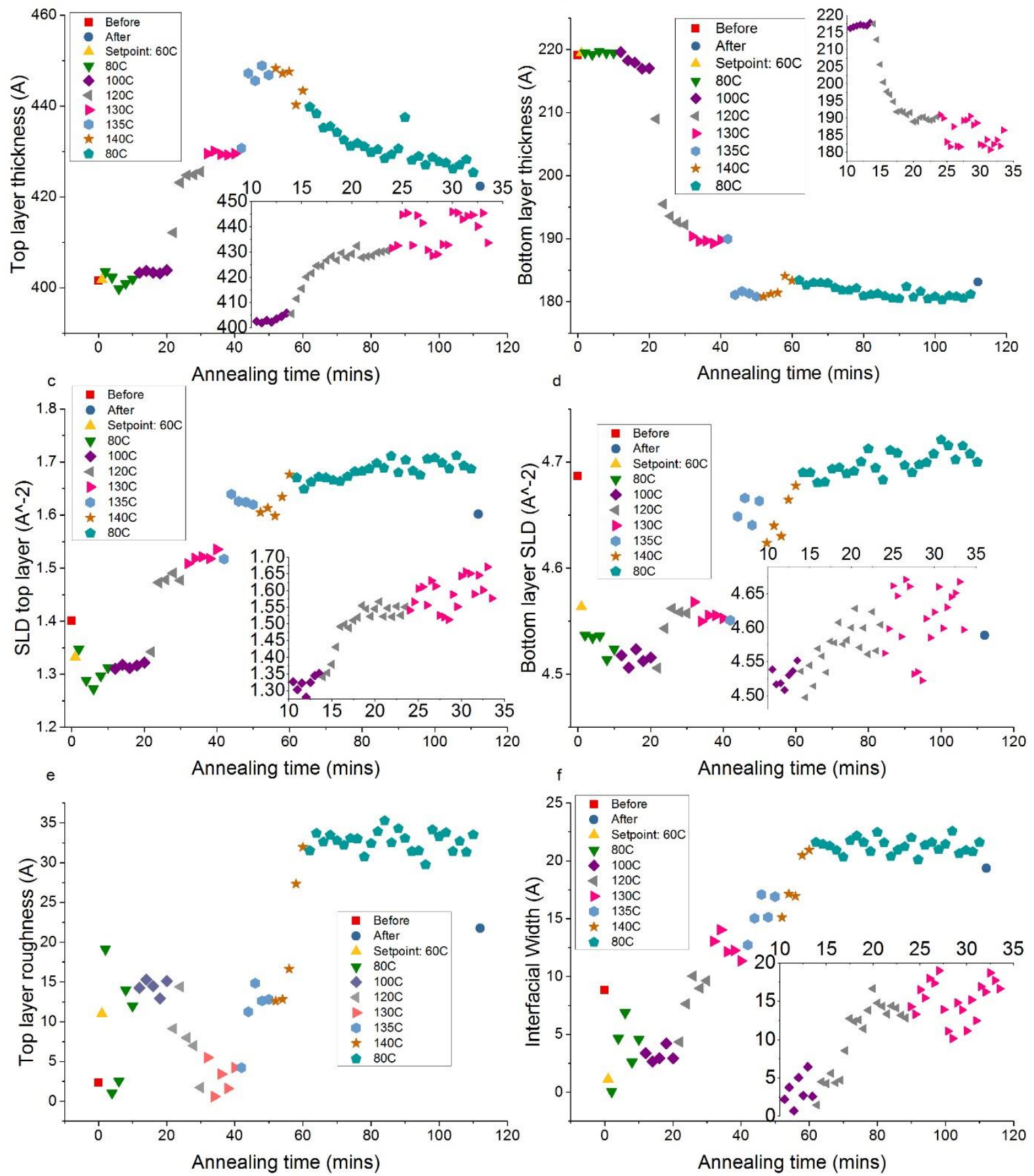

Figure 4.1-25 The change in fit parameters over time for PCBM/PS MW=20kDa. Each data point is for reflectivity summed over four 30s measurements barring the inset graphs which show data points for individual 30s measurements. Graph a is thickness of the top layer (PS-rich), graph b is the thickness of the bottom layer (PCBM), graph $c$ is the SLD of the top layer, $d$ is the SLD of the bottom layer, $e$ is the top layer roughness and $f$ is the interfacial roughness. 


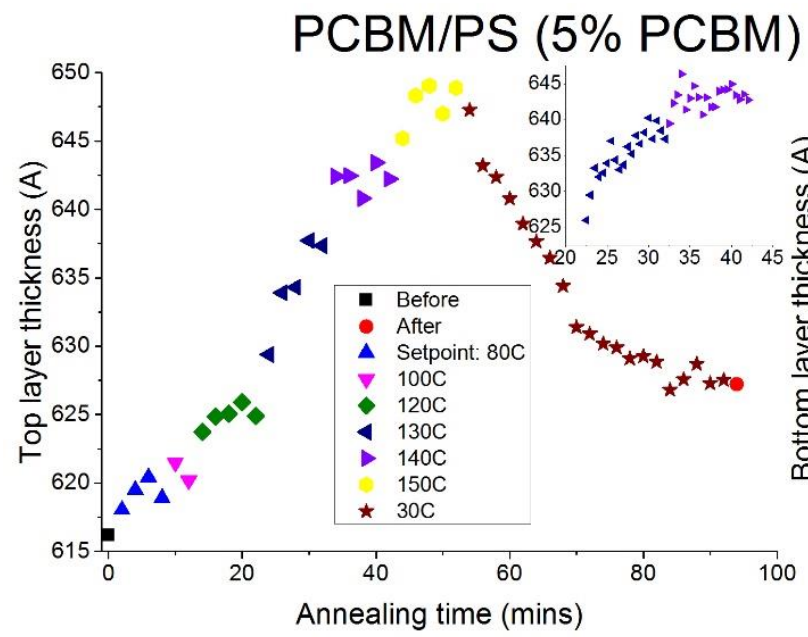

$\mathrm{MW}=100 \mathrm{k} \mathrm{Da}$ in-situ fit paramete
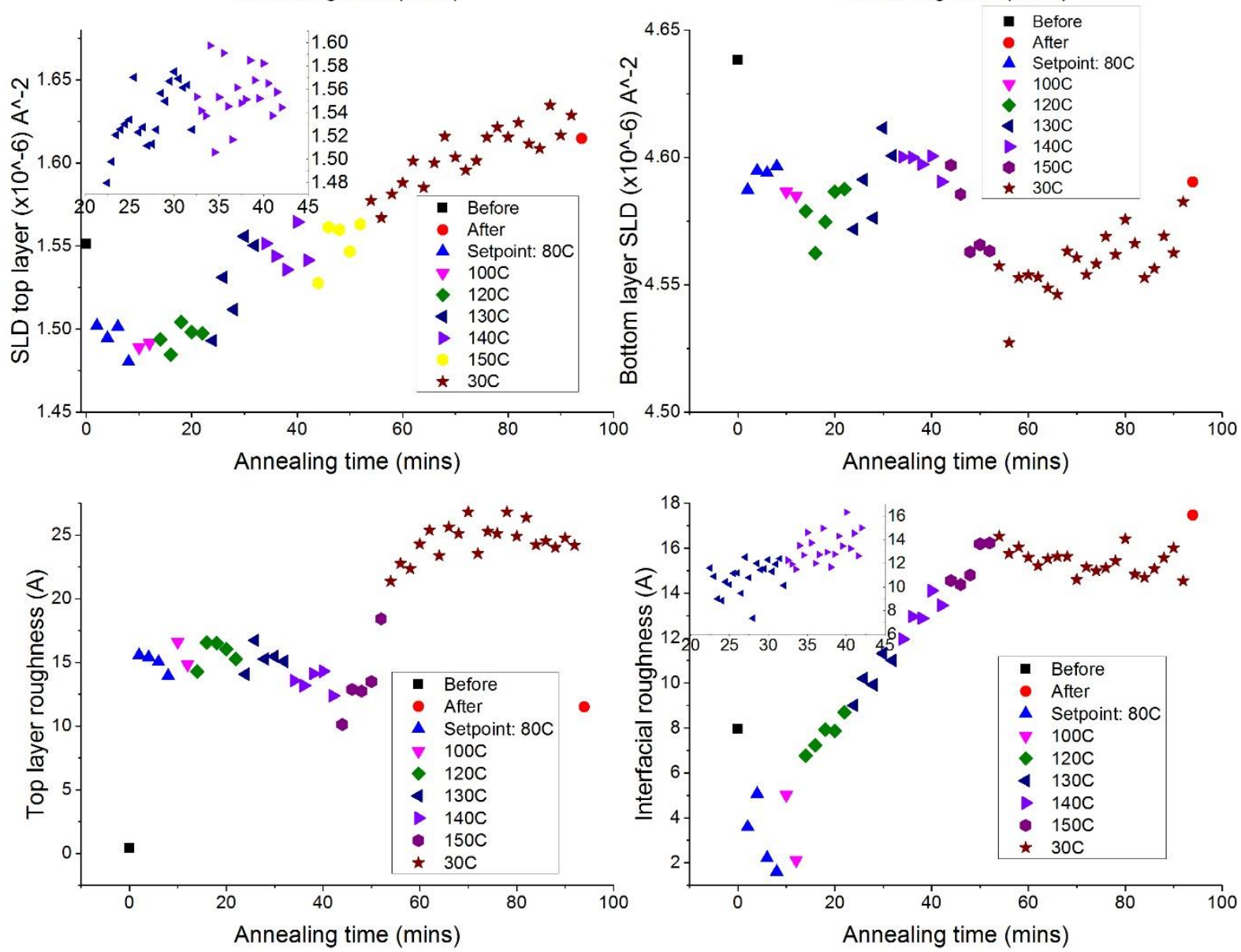

Figure 4.1-26: The change in fit parameters over time for PCBM/PS MW=100kDa with the top layer having an initial concentration of 5\% PCBM v/v.. Each data point is for reflectivity summed over four 30 s measurements barring the inset graphs which show data points for individual 30s measurements. Graph a is thickness of the top layer (PS-rich), graph $b$ is the thickness of the bottom layer (PCBM), graph $c$ is the SLD of the top layer, $d$ is the SLD of the bottom layer, $e$ is the top layer roughness and $f$ is the interfacial roughness. 

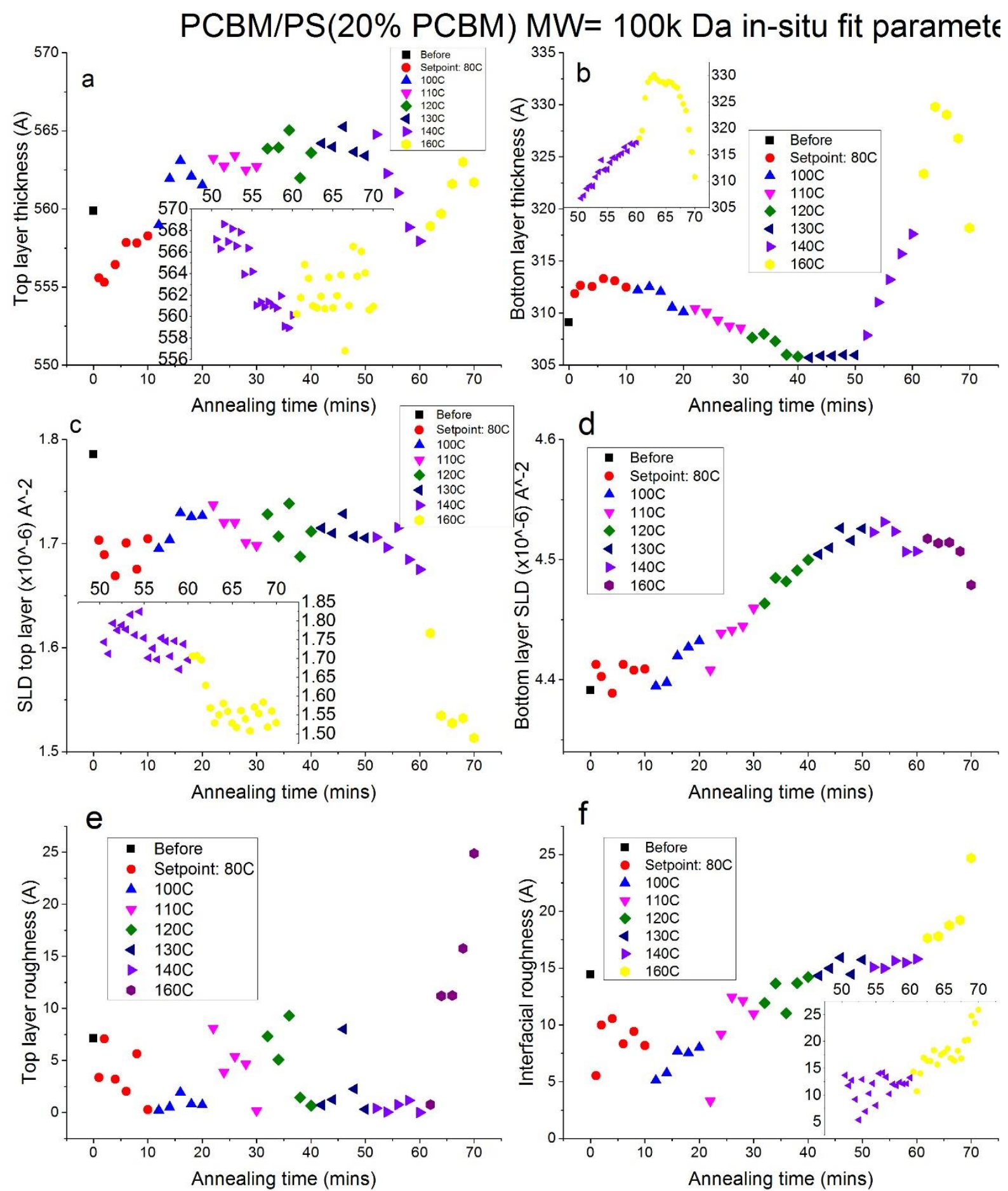

Figure 4.1-27:The change in fit parameters over time for PCBM/PS MW=100kDa with the top layer having an initial concentration of 20\% PCBM v/v. Each data point is for reflectivity summed over four 30 s measurements barring the inset graphs which show data points for individual 30s measurements. Graph a is thickness of the top layer (PS-rich), graph b is the thickness of the bottom layer (PCBM), graph $c$ is the SLD of the top layer, $d$ is the SLD of the bottom layer, $e$ is the top layer roughness and $f$ is the interfacial roughness. 

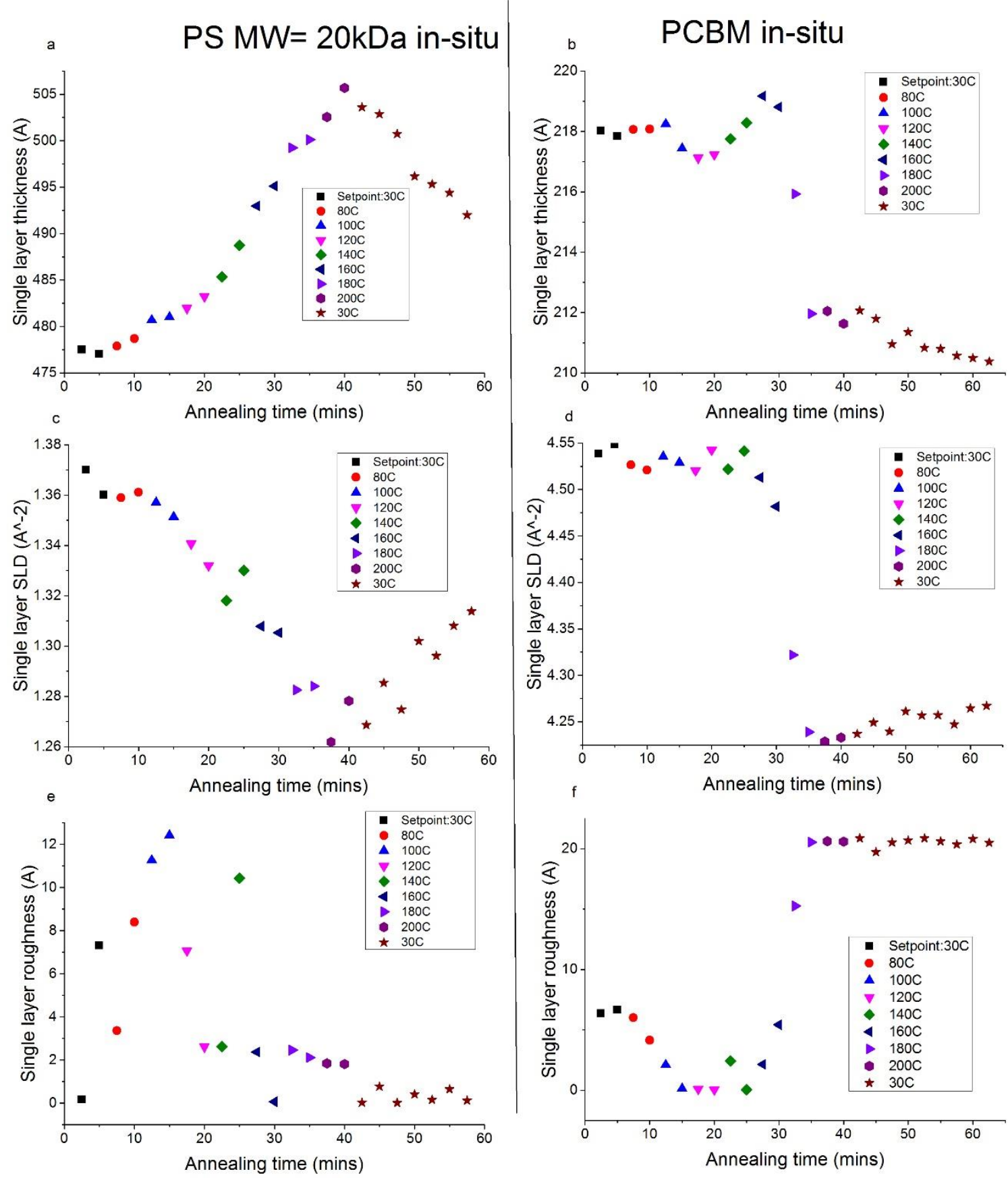

Figure 4.1-28 :The change in fit parameters over time for a PCBM single layer on the right and a PS MW=20kDa single layer on the left. Each data point is for reflectivity summed over four or five 30s measurements. Graph a is thickness of the $P S$ single layer, graph $b$ is the thickness of the PCBM single layer, graph $c$ is the SLD of the PS single layer, $d$ is the SLD of the PCBM single layer, e is the PS single layer roughness and $f$ is the PCBM single layer surface roughness.

Figure 4.1-22 to Figure 4.1-28 show the change in the parameter values when fitted to data in which the reflectivity was summed over four consecutive 30 second 
measurements, with the inset graphs showing data points for each 30 second measurement in the region at which the parameters changed significantly. Thirtysecond single angle measurements capture the kinetics of the in-situ samples yet sacrifice the accuracy of the fits as the higher Q, lower reflectivity regions, are ignored and the error bars are larger as there are fewer counts. The chi-squared contour map in the appendix (Figure 4.1-42 and Figure 4.1-43) shows that there is no strong correlation between interfacial and surface roughness (with fits much more sensitive to the interface roughness).

For all samples we see an increase in the top layer thickness (except for the $20 \%$ by volume PCBM:PS top layer) at the same time as an increase in the SLD of the top layer and a decrease of the thickness of the bottom layer. These thickness changes (comparing samples before and after annealing) are substantial. We see a change of $13 \%$ and $36 \%$ for the PS and PCBM layer thickness respectively for both the $2 \mathrm{k}$ and 3.5k samples, as in Figure 4.1-22 and Figure 4.1-23. For the 5k and 20k PS layers, we see a change of $7 \%$ in the top layer and $20 \%$ and $17 \%$ in the bottom layers respectively (see Figure 4.1-24 and Figure 4.1-25). For the 5\% by volume PCBM:PS blended top layer we see a top layer change in thickness of $2 \%$ and a bottom layer change in thickness of 9\% (Figure 4.1-26). As can be seen in Figue 4.1-20, all of these thickness changes conserve the total sample thickness well. When looking at layer thicknesses at elevated temperature during annealing, the effects of thermal expansion must be considered. These are portrayed in the single layer data for both PCBM and PS (MW=20k), shown in Figure 4.1-28. There is an expansion of around $2 \%$ for PS between room temperature $/ 80^{\circ} \mathrm{C}$ and the maximum in-situ bilayer annealing temperature of $160{ }^{\circ} \mathrm{C}$, and a similar contraction on cooling. PCBM shows minimal (sub 1\%) expansion over this range. The only observable change in the bilayer samples during cooling is a reduction in the thickness of the top layer which is in line with the thermal contraction seen for the single PS layer. The fact that there are no significant changes to the composition profiles (layer compositions of interfacial roughness) on cooling corroborates the ex-situ measurements. Both approaches (rapid quenching ex-situ or slow in-situ cooling) give very similar SLD profiles, lending further weight to the argument that the system rapidly forms a liquid-liquid equilibrium. 
Figure 4.1-22 shows the evolution of the parameters for the $2 \mathrm{k}$ sample showing a distinct change at $100^{\circ} \mathrm{C}$. Figure $4.1-23$ shows the parameter values for the $3.5 \mathrm{k}$ sample which exhibits a change at $100^{\circ} \mathrm{C}$ and $120^{\circ} \mathrm{C}$. Figure $4.1-24$ shows a change for the $5 \mathrm{k}$ sample at $120^{\circ} \mathrm{C}$. Figure $4.1-25$ shows a change in fitted parameters for the $20 \mathrm{k}$ sample above $120^{\circ} \mathrm{C}$ through $130^{\circ} \mathrm{C}$ to $135^{\circ} \mathrm{C}$. The increase in the temperature at which significant PCBM diffusion is first seen, with PS MW, indicates that the process is influenced by the (MW-dependent) mobility of the polymer, rather than just the Tg of the PCBM (which is above that of the PS).

Figure 4.1-26 shows the sample with initially 5\% PCBM in the top layer, this sample has a slower evolution toward equilibrium; occurring above $130^{\circ} \mathrm{C}$ through to $150^{\circ} \mathrm{C}$. This slower evolution is most likely due to the similar initial concentrations of the initial bilayer and the equilibrium composition (10\%). Figure 4.1-27 shows, similarly, a gradual change above $140^{\circ} \mathrm{C}$ through to $160^{\circ} \mathrm{C}$. There are significant steps in the fit parameter values of samples (most clearly in the $\mathrm{MW}=3.5 \mathrm{k}$ Da sample) as the temperature is raised. These steps (rather than everything occurring at a single temperature) could be interpreted as indicating the existence of a temperaturedependent composition profile. Some parameter values also appear to be correlated as in Figure 4.1-25 where abrupt changes in the values seen at 40 minutes is unphysical and most likely due to the fit moving between two similar solutions, both with a low chi-squared value.

To probe a hypothesis of temperature-dependent composition profiles for the $3.5 \mathrm{k}$ sample we plot the sample surface temperature on top of the bottom layer thickness measurements for this sample in Figure 4.1-29. The colours of the data points show the setpoint temperature from which the sample surface temperature can be estimated. The most obvious explanation for changes in parameters at more than one temperature is that the overshoot in sample surface temperature for the PCBM/PS $3.5 \mathrm{k}$ sample allowed the composition to evolve briefly at a setpoint of $100^{\circ} \mathrm{C}$ and then fully at a setpoint of $120^{\circ} \mathrm{C}$. The step in the thickness of the bottom layer for a PCBM/PS 3.5k in Figure 4.1-23 could be explained by the overshoot in temperature meaning that perhaps the diffusion of PCBM only occurs above $105^{\circ} \mathrm{C}$ and that the system simply didn't have time to fully equilibrate when the set-point changed to $100^{\circ} \mathrm{C}$ as shown in Figure 4.1-29. 
In contrast to the ex-situ experiments (in which the sample surface temperatures increase rapidly and then stabilise without overshoot), it appears that these temperature overshoots (particularly at around the polymer $\mathrm{Tg}$ ) can give the appearance of temperature-dependent composition profiles, which is in fact just an artefact of the annealing methodology.

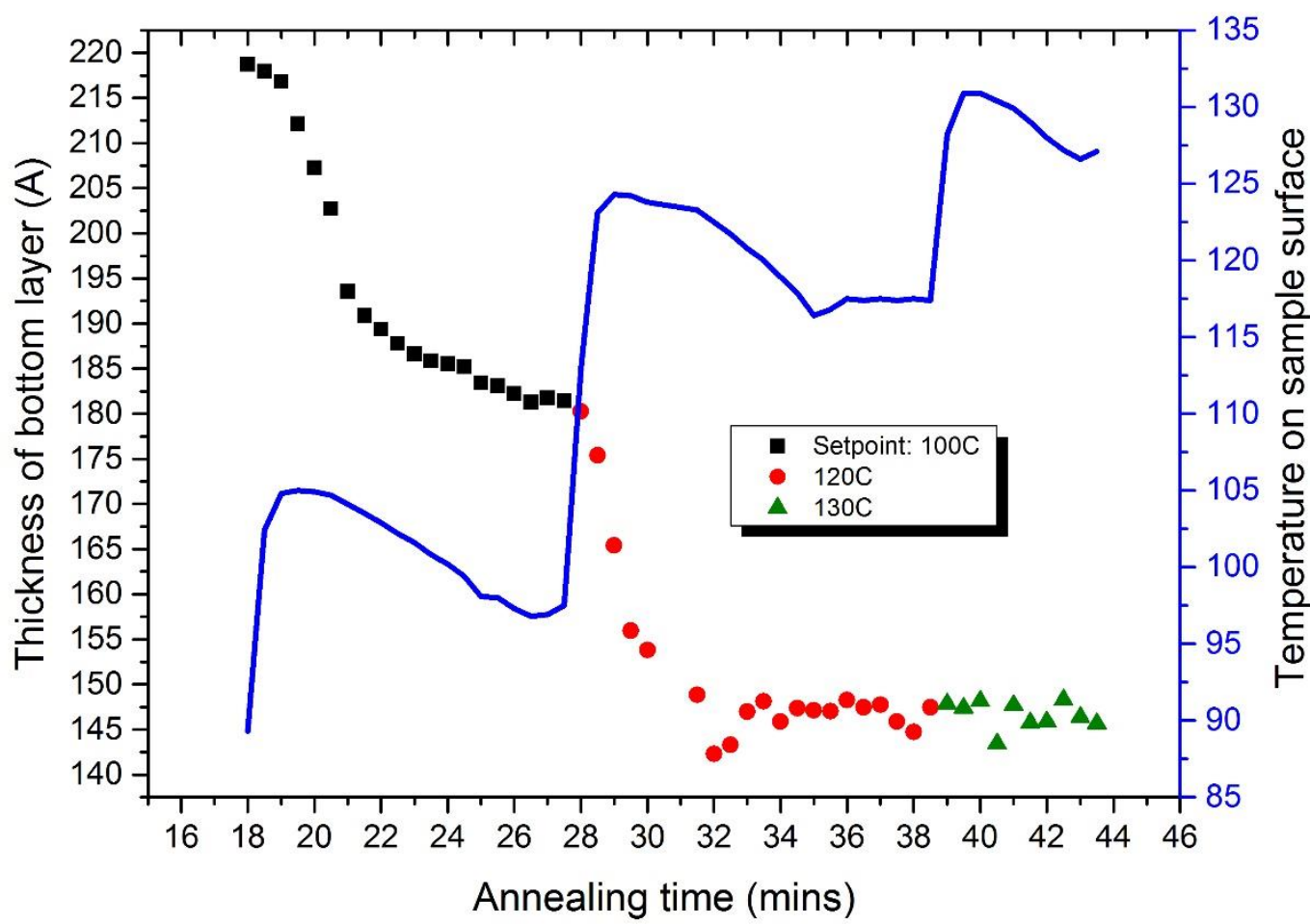

Figure 4.1-29: The temperature on the sample surface overlaid against the step in thickness of the bottom layer for PCBM/PS MW=3.5kDa.

In the in-situ data for the $\mathrm{MW}=2 \mathrm{k}$ and $3.5 \mathrm{k}$ Da there was a change in composition below the bulk experimental glass transition for PCBM. There is a reduced glass transition temperature for lower molecular weights, in the case of polystyrene as discussed in Section 2.3. A reduced glass transition has not been observed in PCBM but the enhanced mobility at the interface caused by the presence of the amorphous polystyrene, as proposed by Mon et al, could explain the diffusion of the PCBM into the top layer despite being below its bulk glass transition temperature. The reported glass transition temperature of PCBM is $118^{\circ} \mathrm{C}$ degrees (Ngo, Nguyen, and Nguyen 2012). The bulk glass transition temperature of PS is $107^{\circ} \mathrm{C}$ (Rieger 1996) with depression in this value observed as in Figure 2.3-2 for low MW. Using equation (2.11) the system can be expected to evolve at $109^{\circ} \mathrm{C}$ for an approximate volume 
fraction of $25 \%$ PCBM across the bilayer. Using the calibration curves in section 3.12 it is seen that at the setpoint temperature of $100^{\circ} \mathrm{C}$ there is some overshoot above this value. The samples which evolve slowly above higher temperatures indicate that there may not have been sufficient overshoot at $100^{\circ} \mathrm{C}$ (most samples only evolved above $120^{\circ} \mathrm{C}$ ). Very slow rate of evolution is seen in the samples with a blended top layer.

With the PCBM single layer, we see a contraction at $180^{\circ} \mathrm{C}$, perhaps due to some crystallisation and roughening of the PCBM. However, it may be due to correlation between fit parameters as the thickness and SLD both go down at the same time. This annealing temperature of $180^{\circ} \mathrm{C}$ is not relevant for the bilayer samples.

The longest relaxation times (reptation-times) for entangled PS chains at the various temperatures (sample surface temperatures) used for annealing were calculated. This was done using experimental data from the literature (Bent et al, Science, 301, 16911695), which was then scaled using the reptation-time-versus-MW relationship (proportional to $\mathrm{MW}^{3.4}$, for entangled polymers) and the Williams-Landel-Ferry (WLF) equation for the temperature-dependence (R. Colby; M. Rubinstein; 2003; R. A. L. Jones 2002)

Apart from the reptation time for $278 \mathrm{k}$ PS at $152^{\circ} \mathrm{C}$ (which was $\sim 1$ minute) these relaxation times are all less than 10 seconds. This time scale is much shorter than the shortest annealing times used. Byway of example, at $152{ }^{\circ} \mathrm{C}$ the reptation time of $111 \mathrm{k}$ PS is 2.4 seconds, while an $18.5 \mathrm{k}$ PS molecule, which is only just longer than the entanglement MW of $\sim 18 \mathrm{k}$ (R. Colby; M. Rubinstein; 2003), has a reptation time of $\sim 5$ milliseconds at $152{ }^{\circ} \mathrm{C}$ and 9 seconds at $117{ }^{\circ} \mathrm{C}$. The unentangled lower MWs $(2 \mathrm{k}, 3.5 \mathrm{k}$ and $5 \mathrm{k})$ used in the thesis will have shorter relaxation times than these. 


\subsubsection{The influence of PS MW on interfacial roughness and coexisting compositions.}

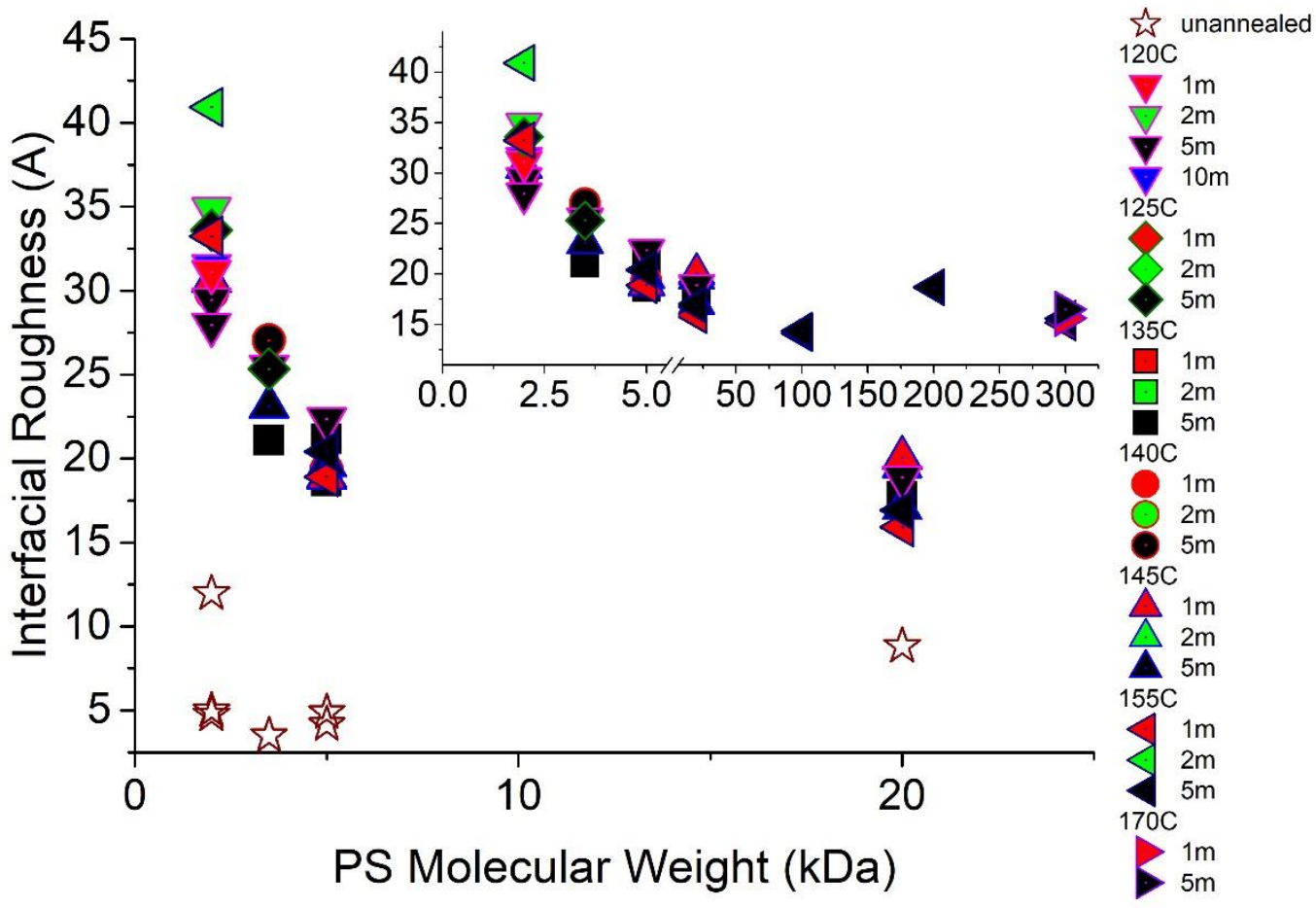

Figure 4.1-30: Gaussian interface roughness versus PS molecular weight for PCBM/PS bilayers annealed at various temperatures and times for ex-situ samples.

Figure 4.1-30 shows the measured values for interfacial roughness across a broad range of molecular weights for a range of ex-situ annealing times and temperatures as indicated by the legend. This figure combines the data from all ex-situ annealed samples, except for four samples, that were rejected for reasons of sample/fit quality that are explained in the appendix to this chapter. It is clear by eye that there is no obvious dependence on temperature and that there is a strong dependence on molecular weight. The $\mathrm{MW}=2 \mathrm{k}$ Da samples have up to double the interfacial roughness of the higher molecular weights.

In Figure 4.1-30 the experimental values include any lateral roughness, due to thermal capillary waves, so these measurements represent an upper-bound for the intrinsic interfacial width, due to molecular mixing. Although, as discussed previously, no significant Yoneda peak was observed, indicating that capillary-wave roughness is likely to be small. Qualitatively, an upturn in the interfacial width at around $\mathrm{MW}=5 \mathrm{kDa}$ is seen for both the theory and the experimental measurements (see Figure 4.1-30 above and Figure 2.6-2 and 2.6-3 in Section 2.6). 


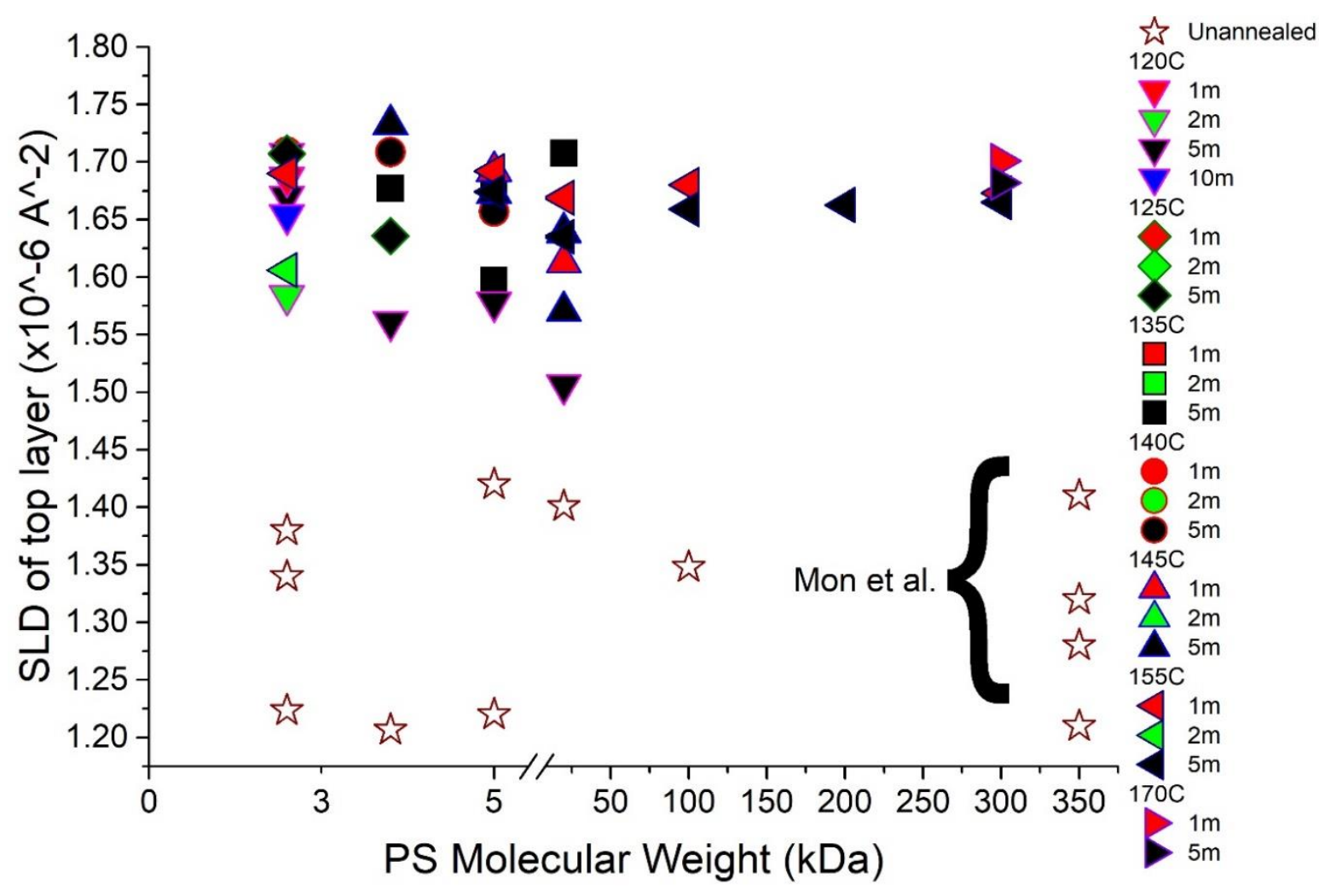

Figure 4.1-31: The SLD of the top PS rich layer is shown for all of the molecular weight series of PCBM/PS bilayers from ex-situ samples of varying MW. Data points from reference Mon et. al(Môn et al. 2015)

Figure 4.1-31 shows the top layer SLDs over a range of MW and includes the unannealed values that we measured by NR (again combining all ex-situ samples, except for the four rejects). The annealed values are consistently above the unannealed values and the average of these values, represents a volume fraction of $10.3+/-1.1 \%$ PCBM in the PS-rich layer although it can be seen from the graph that there is some spread in these values. 


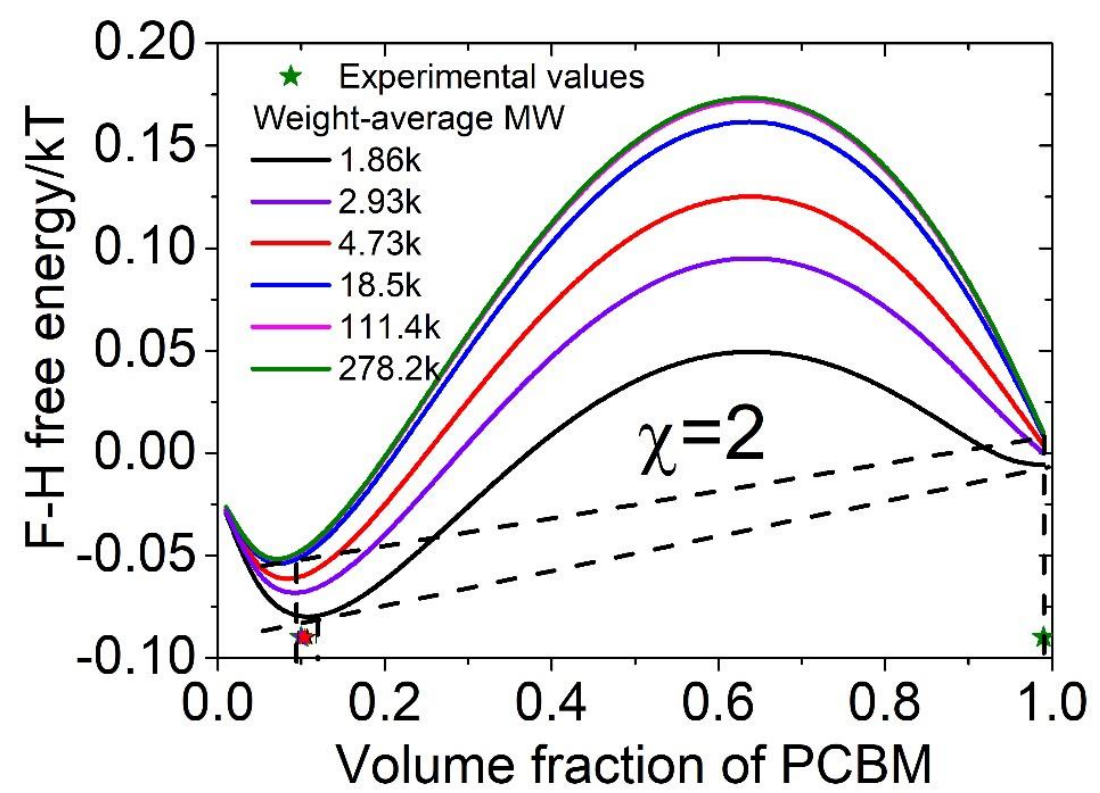

Figure 4.1-32: Free energy curve for an $\mathrm{F}-\mathrm{H}$ chi value of 2 for a polymer mixture with monomer size being that of a PCBM molecule. The dashed lines show the commom tangent construction for $2 k$ and $20 k-300 k P S$ and the stars indicate experimentally measured compositions.The PS MWs in the legend are weight averages. $M W$ of $1.86 k$ corresponds to $N=2.9 ; 2.93 k$ to $N=4.6 ; 4.73 k$ to $N=7.5$; $18.5 k$ to $N=29.2 ; 111.4 k$ to $N=176$; and $278.2 k$ to $N=440$.

Flory-Huggins theory applies to polymer-small-molecule systems and it can be used to describe PCBM/PS by taking the lattice size to be the volume of a PCBM molecule, which was calculated to be $1 \mathrm{~nm}^{3}$. This was found by taking the mean unannealed PCBM SLD $\left(4.65+/-0.02 \times 10^{-6} \AA^{-2}\right)$ and using the NIST calculator (Kienzle 2016) to work out the density corresponding to this SLD to be $1.55 \mathrm{~g} / \mathrm{cm}^{3}$. Then dividing the molecular weight by this density gives the molar volume. Dividing the molar volume by Avogadro's number gives the volume of a single molecule. The number of monomers in a PS molecule of a particular MW is the volume of the PS molecule (calculated using a density of $1.04 \mathrm{~g} / \mathrm{cm}^{3}$ (Wypych 2012)[a range of densities are reported in the literature]) divided by the PCBM molecular volume. Figure 4.1-32 is drawn for an F-H chi interaction parameter value of 2. This has been calculated by choosing a chi-parameter that predicts the experimentally found compositions for the higher MW (above 5k, within error). The free energy curves for the lower MW values $(2 \mathrm{k}, 3.5 \mathrm{k}$ and $5 \mathrm{k})$ are then predictions. The common-tangent construction predicts a slight deviation for lower MW. The data is not sensitive to this predicted change due to the spread in the measured SLD values. The 
compositions (\%PCBM by volume) calculated for the top layer post-annealing are $10.7+/-1.1 \%$ for $2 \mathrm{k}, 10+/-1.3 \%$ for $3.5 \mathrm{k}, 10.4+/-1.1 \%$ for $5 \mathrm{k}$ and $10+/-1.1 \%$ for $20-$ $300 \mathrm{k}$. These compositions, with an average of $10.3 \%$, agree within error with that found by Mon et. al of $9.5+/-1.4 \%$ for $350 \mathrm{k}$ PS.

These compositions were found by comparing the pure measured SLDs and the mixed post-annealing SLDs of each layer as follows

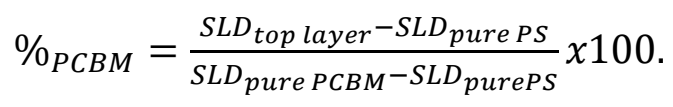

The value for pure PCBM was taken as the average measured from unannealed bilayers as $4.65+/-0.02 \times 10^{-6} \AA^{-2}$. The pure SLD for the PS layer was taken as the average value from unannealed bilayers which was $1.31+/-0.035 \times 10^{-6} \AA^{-2}$.

These results and their implications will be discussed further at the end of chapter 5, together with the results for the bis-PCBM/PS system.

\subsubsection{Appendix}

The justification for fixing the oxide layer

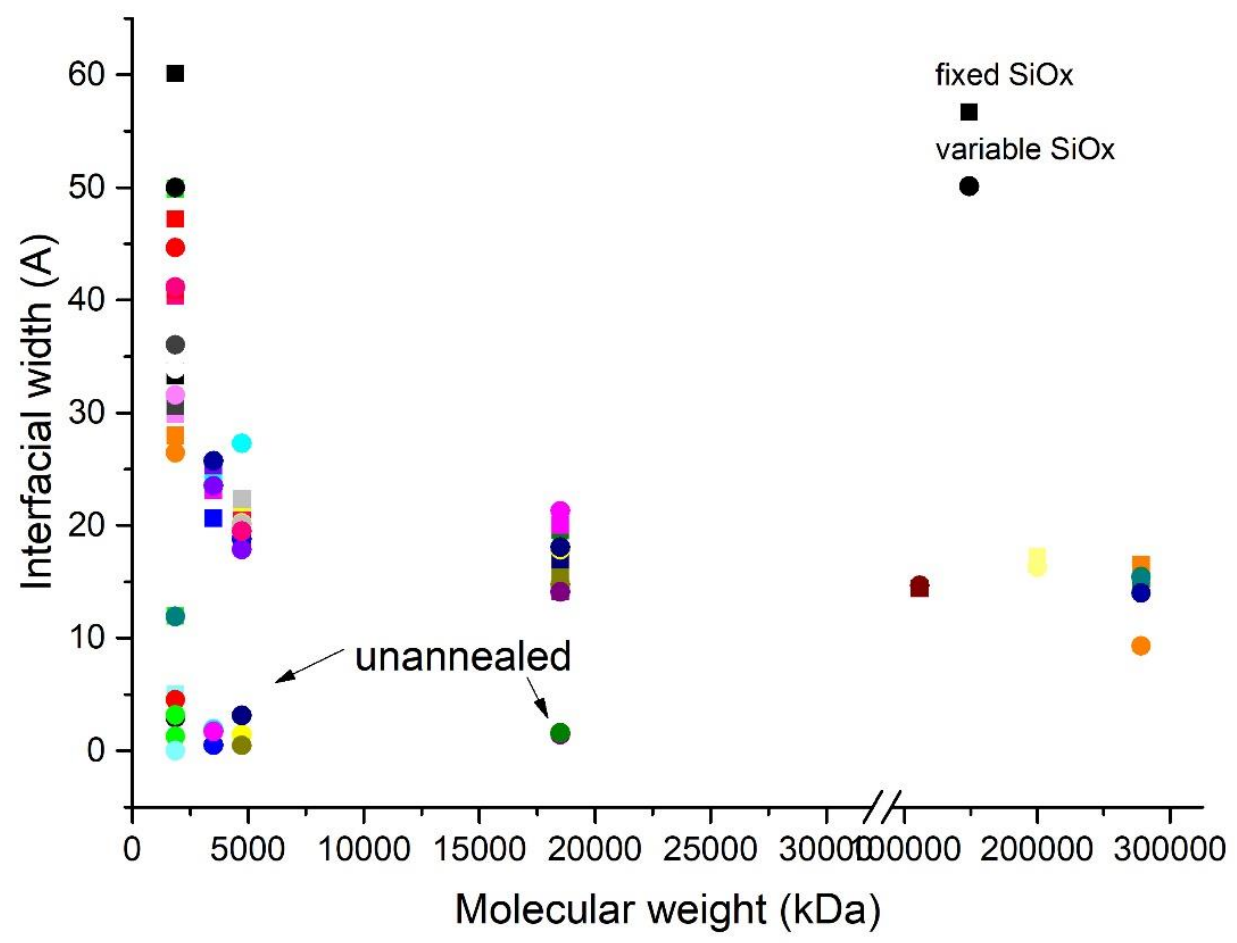

Figure 4.1-33: Parameter values for fixed and varied oxide layers. The same colour represents the same sample. 


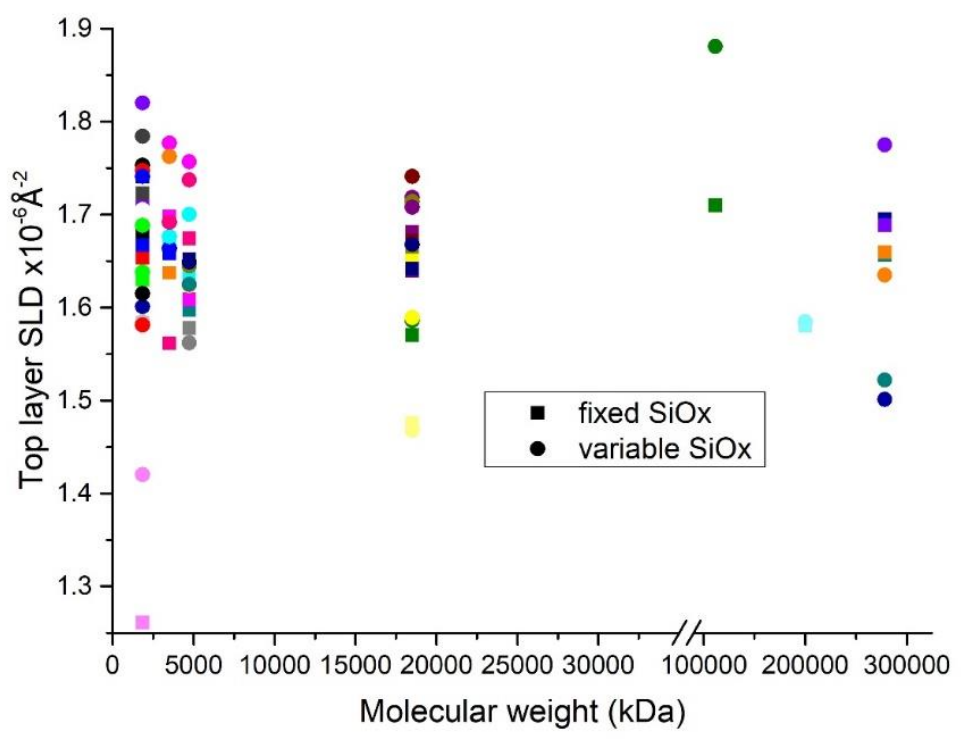

Figure 4.1-34: Parameter values for fixed and varied oxide layers. The same colour represents the same sample.

Figure 4.1-33 and Figure 4.1-34 show the change in the parameters of interfacial width and SLD of the top layer, for fits with a variable or a fixed oxide layer. There is good agreement between these values, with the circles being the value for a varied (from sample to sample) oxide layer while the squares show the values for fixed oxide layers (set as average values of the varied values and set within a given experiment/batch of silicon substrates). Symbols of the same colour represent the same sample. Figure 4.1-35 shows the spread in differences between the fixed and varied oxide layer parameters for all of the samples in this thesis.

Figure 4.1-35 shows the spread in differences between parameter values for fixed and varied oxide layer parameters. This graph shows data for all of the samples in this thesis. 


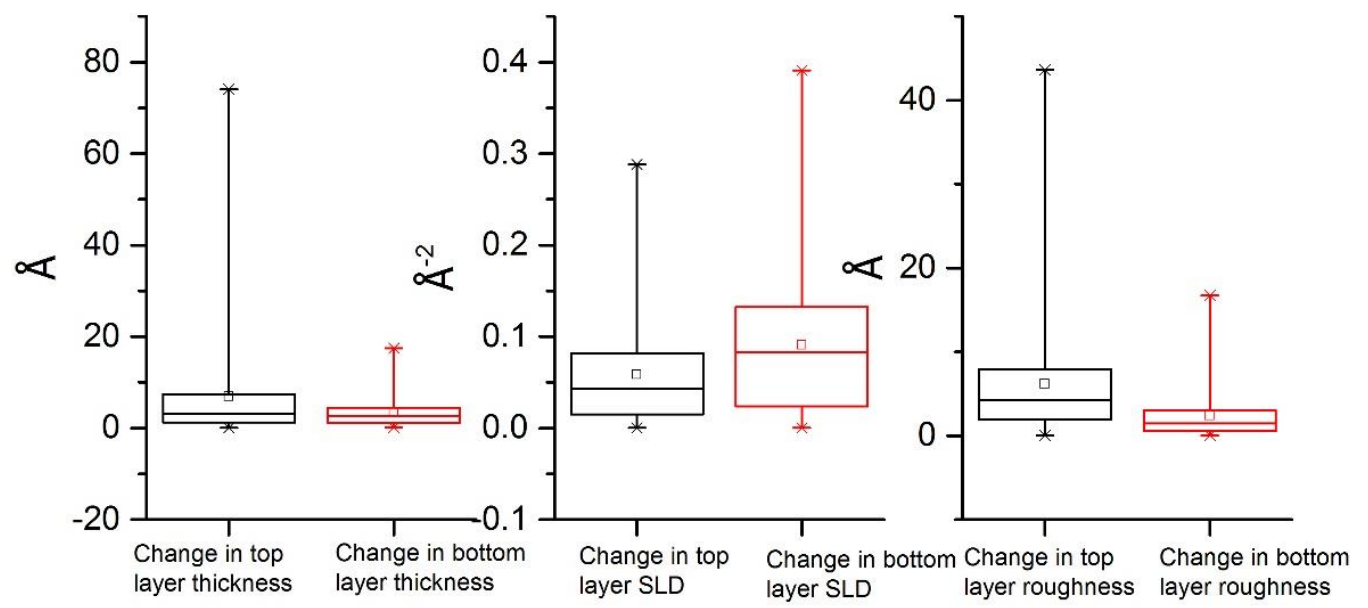

Figure 4.1-35: Box and whisker diagram of the average and spread in the magnitude of the change in parameter values between a fixed and a variable oxide layer for all samples. The whisker ends indicate max and min values, the box edges indicate first (lower) and third (upper) quartiles. The middle line of the box shows the median value, and the squares show the mean value.

\begin{tabular}{|c|c|c|c|c|c|c|c|c|c|}
\hline & $\begin{array}{l}\text { Thick } \\
\text { ness } \\
\text { of the } \\
\text { top } \\
\text { layer } \\
(\AA)\end{array}$ & $\begin{array}{l}\text { SLD } \\
\text { of the } \\
\text { top } \\
\text { layer } \\
(\times \\
10^{-6} \AA \\
)\end{array}$ & $\begin{array}{l}\text { Rough } \\
\text { ness of } \\
\text { the top } \\
\text { layer } \\
(\AA)\end{array}$ & $\begin{array}{l}\text { Thick } \\
\text { ness } \\
\text { of the } \\
\text { bot- } \\
\text { tom } \\
\text { layer } \\
(\AA)\end{array}$ & $\begin{array}{l}\text { SLD } \\
\text { of the } \\
\text { bot- } \\
\text { tom } \\
\text { layer } \\
(\times \\
10^{-6} \AA^{-}\end{array}$ & $\begin{array}{l}\text { Rough } \\
\text { ness at } \\
\text { the } \\
\text { inter- } \\
\text { face } \\
(\AA)\end{array}$ & $\begin{array}{l}\text { Thick } \\
\text { ness } \\
\text { of the } \\
\text { oxide } \\
\text { layer } \\
(\AA)\end{array}$ & $\begin{array}{l}\text { SL } \\
D \\
\text { of } \\
\text { the } \\
\text { ox- } \\
\text { ide } \\
\text { lay } \\
\text { er }\end{array}$ & $\begin{array}{l}\text { Rough } \\
\text { ness of } \\
\text { the } \\
\text { oxide } \\
\text { layer } \\
(\AA)\end{array}$ \\
\hline $\begin{array}{l}120 \\
\mathrm{C} \\
1 \mathrm{~m} \\
\text { fixed } \\
\text { ox- } \\
\text { ide } \\
\text { layer }\end{array}$ & 633 & 1.69 & 15.9 & 129 & 4.64 & 31 & 12 & $\begin{array}{l}2.5 \\
9\end{array}$ & 3 \\
\hline $\begin{array}{l}120 \\
\mathrm{C} \\
1 \mathrm{~m} \\
\text { vari- } \\
\text { able } \\
\text { ox- } \\
\text { ide } \\
\text { layer }\end{array}$ & 633 & 1.69 & 13.4 & 123 & 4.61 & 31.4 & 13 & $\begin{array}{l}3.4 \\
5\end{array}$ & 5.3 \\
\hline $\begin{array}{l}120 \\
\mathrm{C} \\
10 \mathrm{~m} \\
\text { fixed } \\
\text { ox- } \\
\text { ide } \\
\end{array}$ & 656 & 1.58 & 28.2 & 129 & 4.63 & 33.5 & 11.5 & 2.8 & 3 \\
\hline
\end{tabular}




\begin{tabular}{|l|l|l|l|l|l|l|l|l|l|}
\hline layer & & & & & & & & & \\
\hline $\begin{array}{l}120 \\
\text { C } \\
10 \mathrm{~m} \\
\text { vari- } \\
\text { able } \\
\text { ox- } \\
\text { ide } \\
\text { layer }\end{array}$ & 656 & 1.58 & 28.1 & 125 & 4.65 & 34 & 13.1 & 3.4 & 0.006 \\
\hline $\begin{array}{l}125 \\
\text { C } \\
\text { 5m } \\
\text { fixed } \\
\text { ox- } \\
\text { ide } \\
\text { layer }\end{array}$ & 650 & 1.7 & 20.9 & 260 & 4.57 & 33 & 11.5 & 2.8 & 3 \\
\hline $\begin{array}{l}125 \\
\text { C } \\
\text { 5m } \\
\text { vari- } \\
\text { able } \\
\text { ox- } \\
\text { ide } \\
\text { layer }\end{array}$ & 648 & 1.63 & 22.2 & 251 & 4.46 & 33.4 & 13.6 & 3.4 & 5.64 \\
\hline
\end{tabular}

Table 7: List of parameter values for PCBM/PS $M W=2 k$ samples with a fixed or varied oxide layer.for the samples in Figure 4.1-4.

\section{Sample List}

Table 8 shows the NR sample list showing which samples were measured at which reflectometers.

\begin{tabular}{|l|l|l|}
\hline INTER & Figaro & $\mathrm{D} 17$ \\
\hline $2 \mathrm{k} 120^{\circ} \mathrm{C} 5 \mathrm{~m}$ double & $2 \mathrm{k}$ unannealed & $2 \mathrm{k} 135^{\circ} \mathrm{C} 5 \mathrm{~m}$ \\
thickness & $2 \mathrm{k} 140^{\circ} \mathrm{C} 1 \mathrm{~m}$ & $2 \mathrm{k} 140^{\circ} \mathrm{C} 2 \mathrm{~m}$ \\
$2 \mathrm{k} 125^{\circ} \mathrm{C} 5 \mathrm{~m}$ & $2 \mathrm{k} 145^{\circ} \mathrm{C} 1 \mathrm{~m}$ & $2 \mathrm{k} 145^{\circ} \mathrm{C} 2 \mathrm{~m}$ \\
$3.5 \mathrm{k} 125^{\circ} \mathrm{C} 5 \mathrm{~m}$ & $2 \mathrm{k} 155^{\circ} \mathrm{C} 1 \mathrm{~m}$ & $3.5 \mathrm{k} 135^{\circ} \mathrm{C} 5 \mathrm{~m}$ \\
$2 \mathrm{k} 120^{\circ} \mathrm{C} 5 \mathrm{~m}$ & $2 \mathrm{k} 155^{\circ} \mathrm{C} 2 \mathrm{~m}$ & $3.5 \mathrm{k} 140^{\circ} \mathrm{C} 5 \mathrm{~m}$ \\
$3.5 \mathrm{k} 120^{\circ} \mathrm{C} 5 \mathrm{~m}$ & $2 \mathrm{k} \mathrm{unannealed}$ & $3.5 \mathrm{k} 145^{\circ} \mathrm{C} 5 \mathrm{~m}$ \\
$2 \mathrm{k} 125^{\circ} \mathrm{C} 5 \mathrm{~m}$ double & $2 \mathrm{k} 145^{\circ} \mathrm{C} 1 \mathrm{~m}$ & $5 \mathrm{k} 135^{\circ} \mathrm{C} 5 \mathrm{~m}$ \\
thickness & $5 \mathrm{k} \mathrm{unannealled}$ & $5 \mathrm{k} 140^{\circ} \mathrm{C} 5 \mathrm{~m}$ \\
$2 \mathrm{k} 120^{\circ} \mathrm{C} 2 \mathrm{~m}$ & $5 \mathrm{k} 145^{\circ} \mathrm{C} 5 \mathrm{~m}$ & $5 \mathrm{k} 145^{\circ} \mathrm{C} 5 \mathrm{~m}$ \\
$2 \mathrm{k} 120^{\circ} \mathrm{C} 10 \mathrm{~m}$ & $5 \mathrm{k} 155^{\circ} \mathrm{C} 1 \mathrm{~m}$ & $20 \mathrm{k} 135^{\circ} \mathrm{C} 5 \mathrm{~m}$ \\
$5 \mathrm{k} 120^{\circ} \mathrm{C} 5 \mathrm{~m}$ & $20 \mathrm{k} 145^{\circ} \mathrm{C} 1 \mathrm{~m}$ & $20 \mathrm{k} 140^{\circ} \mathrm{C} 5 \mathrm{~m}$ \\
$20 \mathrm{k} 120^{\circ} \mathrm{C} 5 \mathrm{~m}$ & $20 \mathrm{k} 145^{\circ} \mathrm{C} 5 \mathrm{~m}$ & $20 \mathrm{k} 145^{\circ} \mathrm{C} 5 \mathrm{~m}$ \\
$200 \mathrm{k} 155^{\circ} \mathrm{C} 5 \mathrm{~m}$ & $20 \mathrm{k} 155^{\circ} \mathrm{C} 1 \mathrm{~m}$ & PCBM $170^{\circ} \mathrm{C} 2 \mathrm{hrs}$ \\
\hline
\end{tabular}




\begin{tabular}{|l|l|l|}
\hline & $20 \mathrm{k} 155^{\circ} \mathrm{C} 5 \mathrm{~m}$ & $2 \mathrm{k}$ in-situ \\
$100 \mathrm{k}$ & $3.5 \mathrm{k}$ in-situ \\
unannealled & $5 \mathrm{k}$ in-situ \\
$100 \mathrm{k} 155^{\circ} \mathrm{C} 1 \mathrm{~m}$ & $20 \mathrm{k}$ in-stu \\
$100 \mathrm{k} 155^{\circ} \mathrm{C} 5 \mathrm{~m}$ & PCBM 5\% PS 100k in-situ \\
$300 \mathrm{k} 155^{\circ} \mathrm{C} 1 \mathrm{~m}$ & PCBM 20\% PS 100k in-situ \\
$300 \mathrm{k} 155^{\circ} \mathrm{C} 5 \mathrm{~m}$ & \\
$300 \mathrm{k} 170^{\circ} \mathrm{C} 1 \mathrm{~m}$ & \\
$300 \mathrm{k} 170^{\circ} \mathrm{C} 5 \mathrm{~m}$ & \\
$5 \mathrm{k} 145^{\circ} \mathrm{C} 1 \mathrm{~m}$ & \\
$5 \mathrm{k} 155^{\circ} \mathrm{C} 5 \mathrm{~m}$ & \\
PCBM $180^{\circ} \mathrm{C}$ & \\
$2 \mathrm{hrs}$ & \\
\hline
\end{tabular}

Table 8: A list of which samples were measured at which reflectometers where all samples are PCBM/PS bilayers unless otherwise indicated.

\section{Sensitivity of the fit to the adjustable parameters}

Figure 4.1-36 to Figure 4.1-41 show the change in the fitted NR curve as each parameter is varied (with the other 5 parameters fixed at the best-fit values). The chisquared value is shown, indicating how sensitive this is to each parameter. This shows that changes in the bottom layer thickness of $10 \AA$ (a $6 \%$ change), in the top layer thickness of $20 \AA$ (4\%), in the interfacial roughness of $10 \AA$ (47\%), in the top layer roughness of $30 \AA$ (175\%), in the bottom layer SLD of 0.2 (4\%) and in the top layer SLD of $0.4(25 \%)$, all cause an increase in chi-squared of an order of magnitude. 


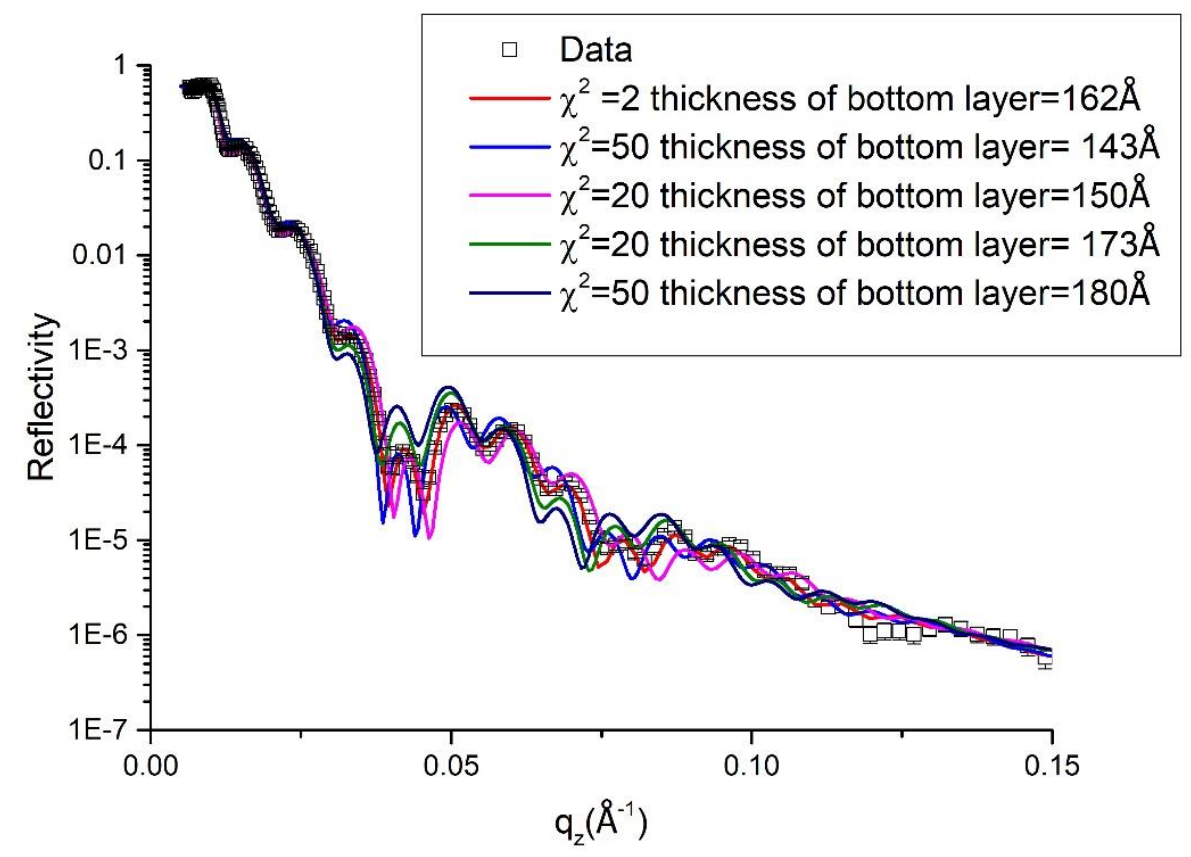

Figure 4.1-36:The change in the fit for altering the value of the bottom layer thickness and keeping the other parameters fixed, which increases the reduced $\chi^{2}$ value by varying degrees. Data shown is for a PCBM/PS MW=5k bilayer annealed at $135 \mathrm{C}$ for $5 \mathrm{~m}$.

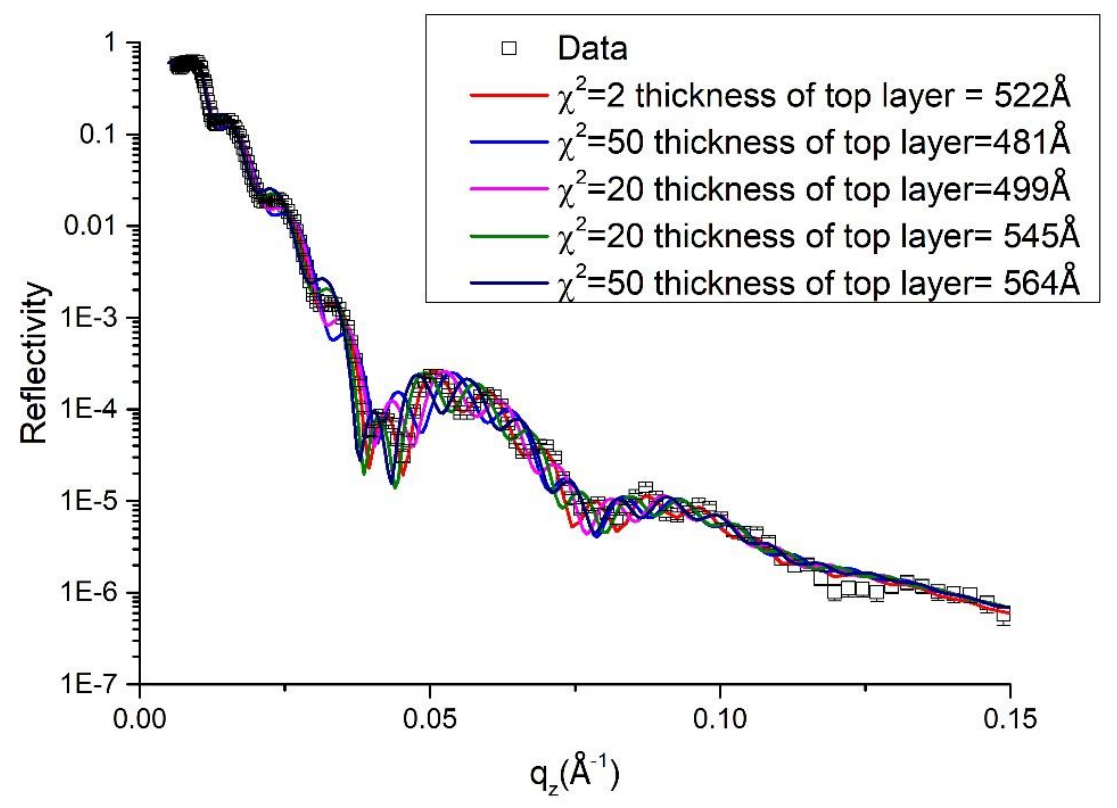

Figure 4.1-37: The change in the fit for altering the value of the top layer thickness and keeping the other parameters fixed, which increases the reduced $\chi^{2}$ value by varying degrees. Data shown is for a PCBM/PS MW=5k bilayer annealed at $135 C$ for $5 \mathrm{~m}$. 


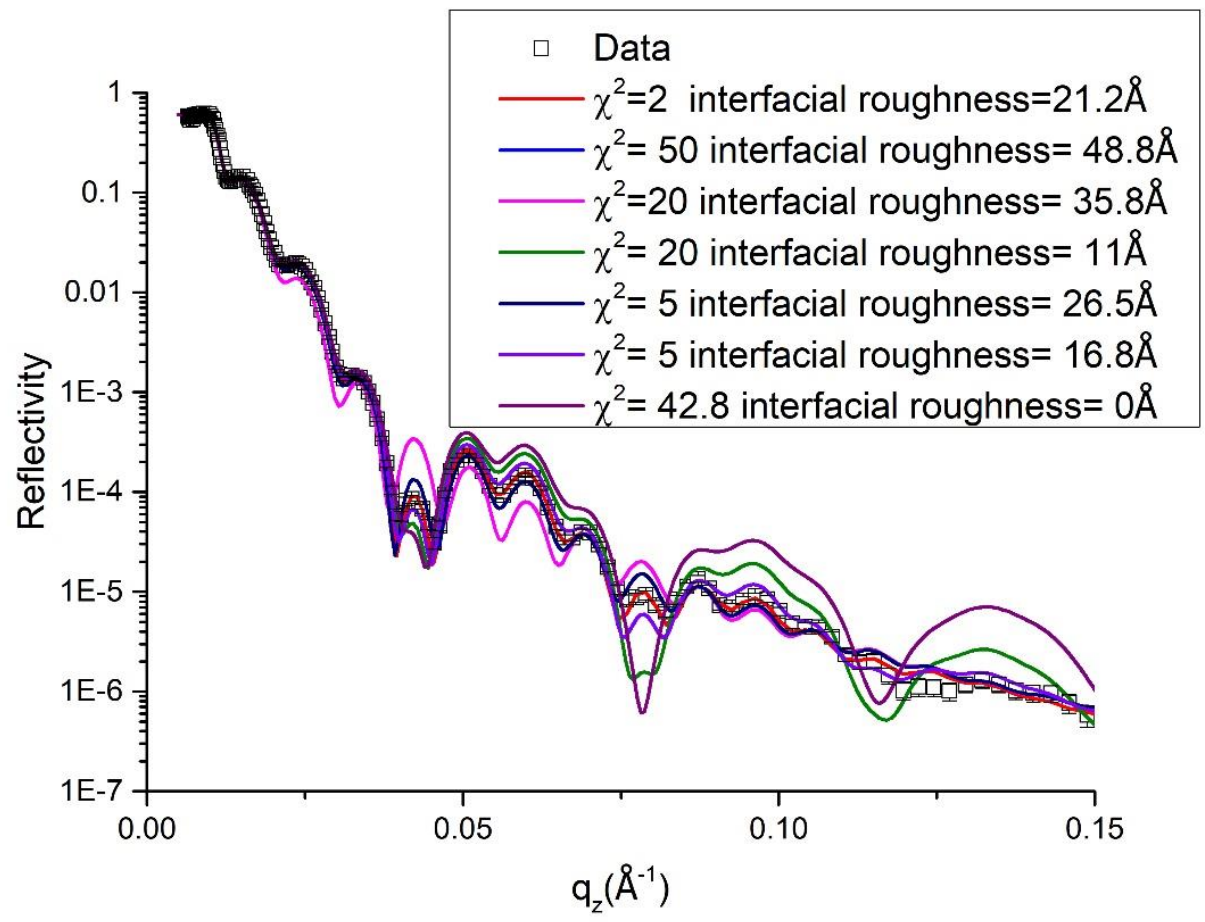

Figure 4.1-38: The change in the fit for altering the value of the interfacial roughness and keeping the other parameters fixed, which increases the reduced $\chi^{2}$ value by varying degrees. Data shown is for a PCBM/PS $M W=5 \mathrm{k}$ bilayer annealed at $135 \mathrm{C}$ for $5 \mathrm{~m}$.

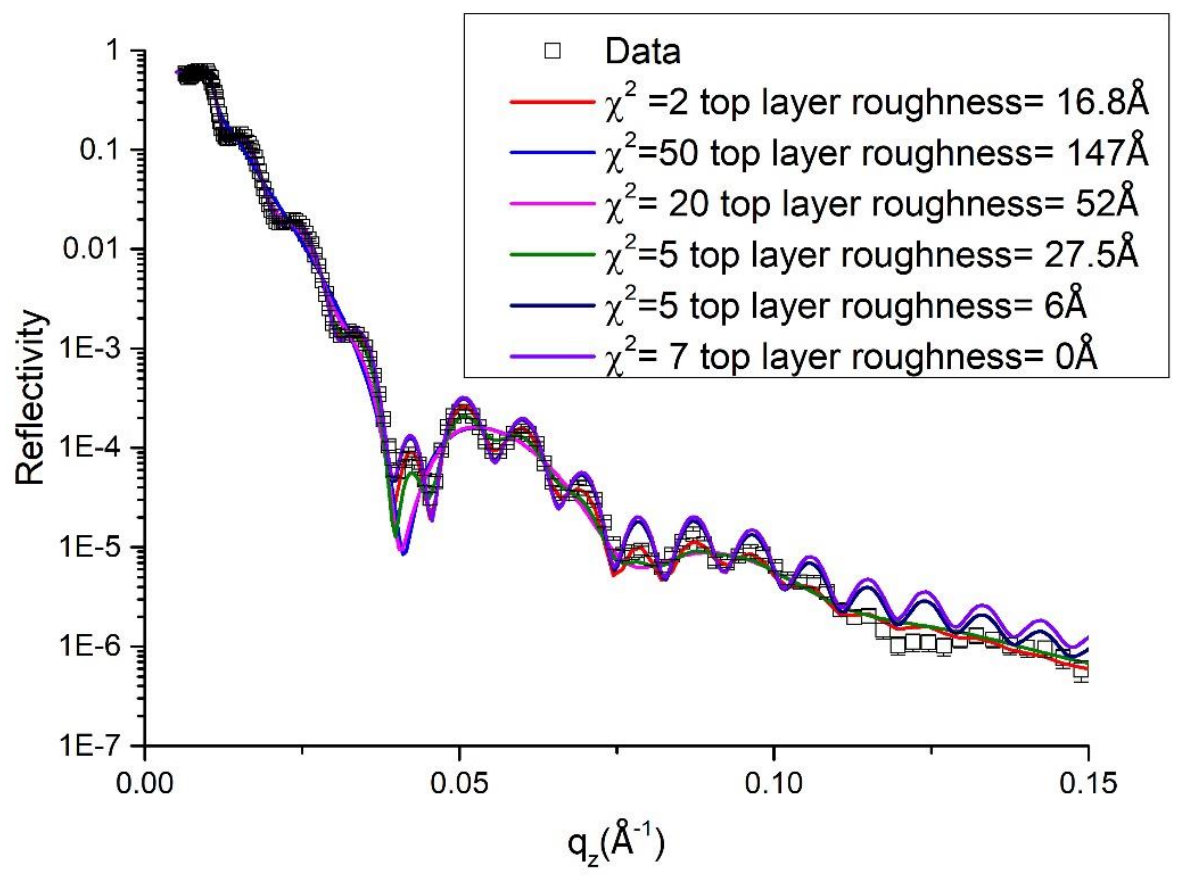

Figure 4.1-39: The change in the fit for altering the value of the top layer roughness and keeping the other parameters fixed, which increases the reduced $\chi^{2}$ value by 
varying degrees. Data shown is for a PCBM/PS $M W=5 k$ bilayer annealed at $135 C$ for $5 \mathrm{~m}$.

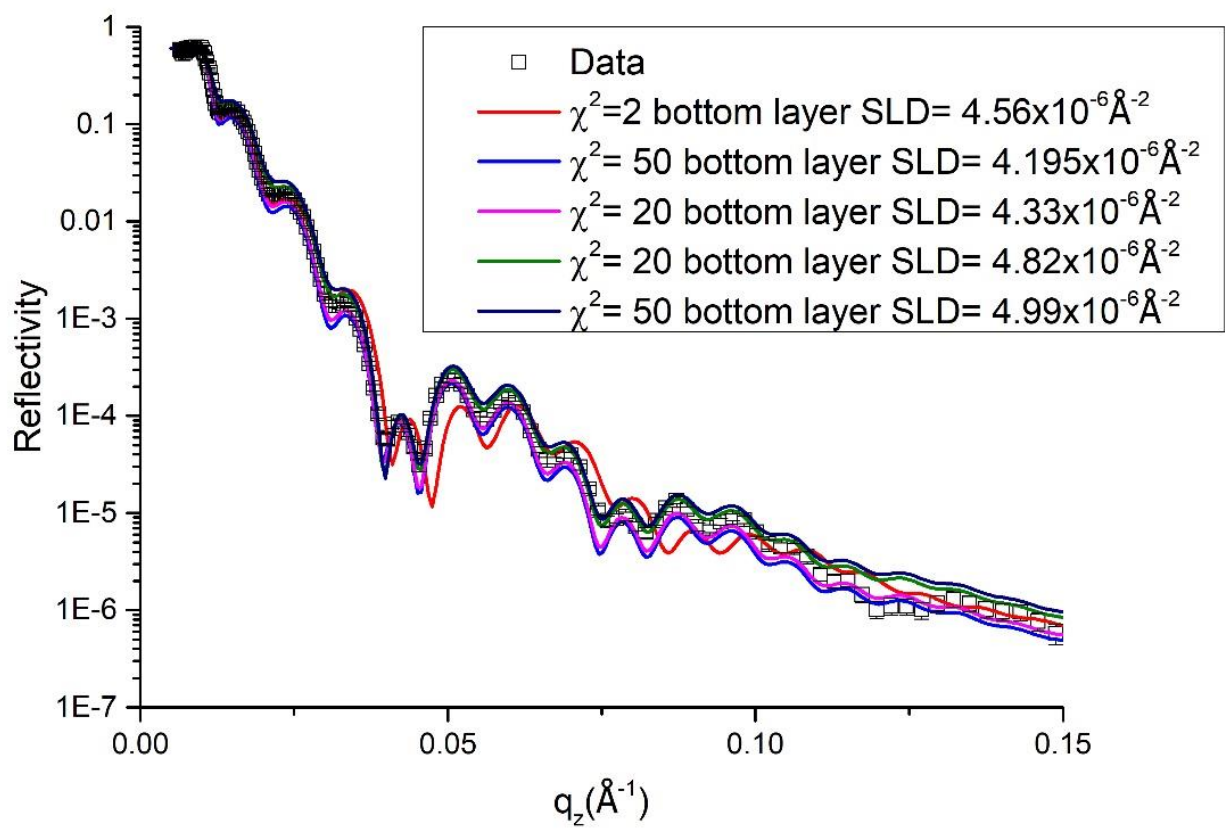

Figure 4.1-40: The change in the fit for altering the value of the bottom layer SLD and keeping the other parameters fixed, which increases the reduced $\chi^{2}$ value by varying degrees. Data shown is for a PCBM/PS MW=5k bilayer annealed at $135 \mathrm{C}$ for $5 \mathrm{~m}$.

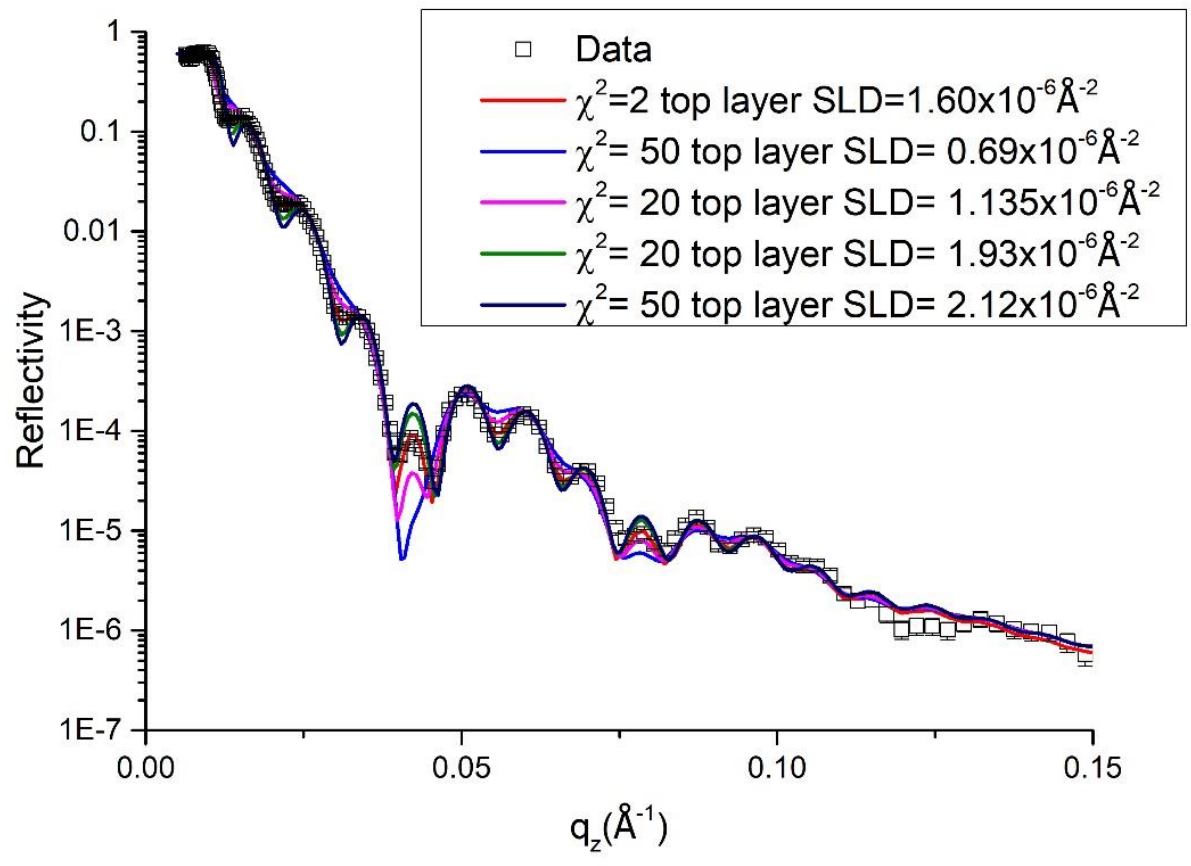


Figure 4.1-41: The change in the fit for altering the value of the top layer SLD and keeping the other parameters fixed, which increases the reduced $\chi^{2}$ value by varying degrees. Data shown is for a PCBM/PS MW=5k bilayer annealed at $135 \mathrm{C}$ for $5 \mathrm{~m}$.

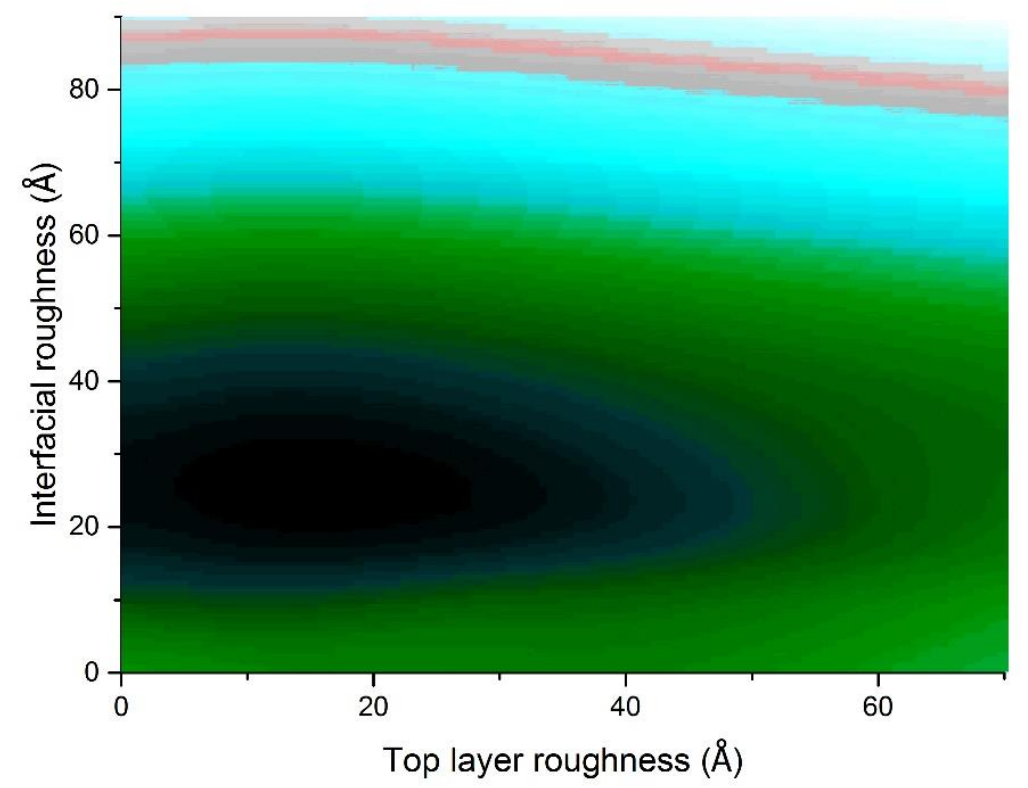

Figure 4.1-42: Chi-squared contour map for top layer versus interfacial roughnesses for PCBM/PS Mw=2k Da sample annealed at $120 \mathrm{C}$ for $5 \mathrm{~m}$. Black shows the minimum in chi-squared. 


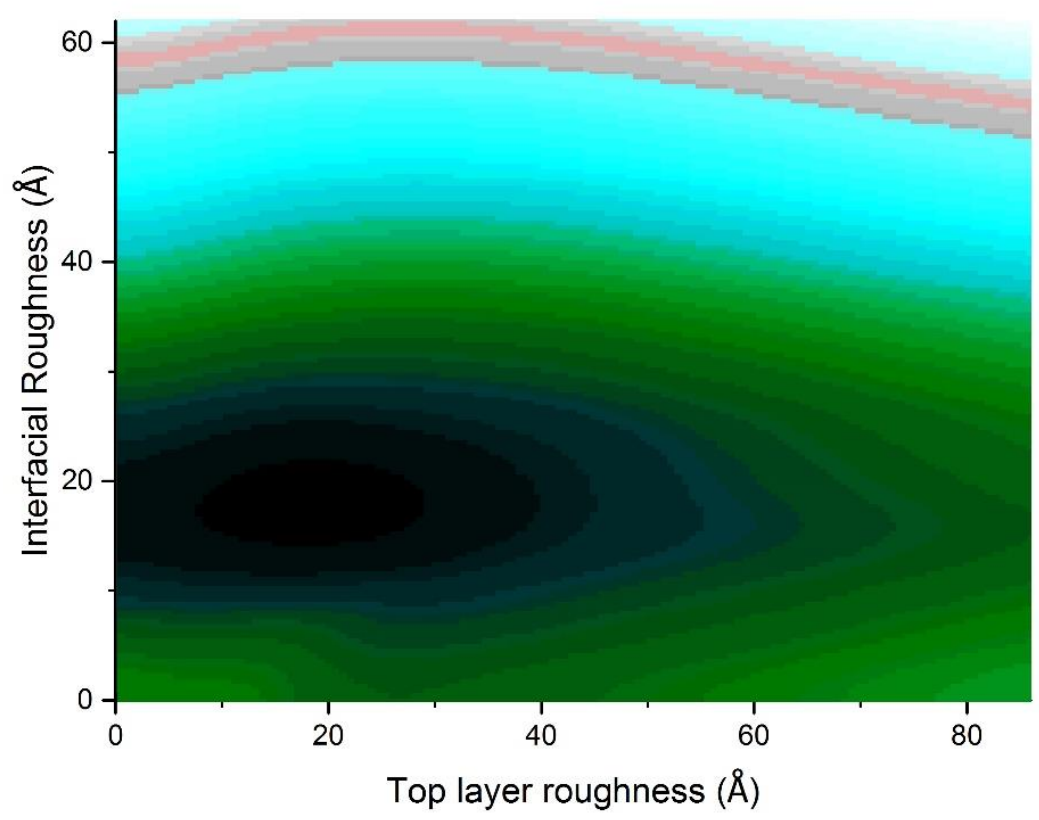

Figure 4.1-43: Chi-squared contour map for top layer versus interfacial roughness for PCBM/PS Mw=20k Da sample annealed at 120C for 5m. Black shows the minimum in chi-squared.

Figure 4.1-42 and Figure 4.1-43 show typical chi-squared contour maps for interfacial and surface roughness. The surface roughness does not affect interfacial roughness strongly although it has a broader minimum suggesting that the fitted values for this parameter are subject to a larger error. The fits are significantly more sensitive to the buried interface roughness between the polymer and fullerene layers.

\section{Film thicknesses for given Spinning speeds and concentration}

Table 9 shows the spinning speeds and concentrations that were used to achieve the thicknesses also shown. The thicknesses were initially measured by AFM using scratch on the sample surface.

\begin{tabular}{|l|l|l|l|}
\hline $\begin{array}{l}\text { Spinning speed } \\
\text { krpm) and con- } \\
\text { centration }(\mathrm{m} / \mathrm{m}) \\
\text { and } \mathrm{MW} \text { of PS }\end{array}$ & $\begin{array}{l}\text { Thickness } \\
\text { measured by } \\
\text { AFM }(\mathrm{nm})\end{array}$ & $\begin{array}{l}\text { Error of AFM } \\
\text { measurement } \\
\text { (standard devia- } \\
\text { tion) }(\mathrm{nm})\end{array}$ & $\begin{array}{l}\text { Thickness meas- } \\
\text { ured by NR of } \\
\text { unannealed bi- } \\
\text { layers (nm) }\end{array}$ \\
\hline $3,2.5 \% 2 \mathrm{k}$ & 54.067 & 0.756 & 55.391 \\
\hline $2,2 \%, 5 \mathrm{k}$ & 56.133 & 0.74944 & 56.332 \\
\hline $3,2.5 \%, 3.5 \mathrm{k}$ & 55.28 & 6.8672 & 62.078 \\
\hline $2,1.5 \%, 20 \mathrm{k}$ & 48.0833 & 0.5634 & 40.16 \\
\hline $3,3 \%, 2 \mathrm{k}$ & 110.75 & 55.74 & 58.185 \\
\hline $1.5,1.5 \%, 100 \mathrm{k}$ & 52.067 & 1.344 & 52.034 \\
\hline Spinning speed & & & \\
\hline
\end{tabular}




\begin{tabular}{|l|l|l|l|}
\hline $\begin{array}{l}\text { (krpm) and con- } \\
\text { centration }(\mathrm{m} / \mathrm{m}) \\
\text { of PCBM }\end{array}$ & & & \\
\hline $3,1.5 \%$ & 21.563 & 6.2884 & 21.734 \\
\hline $3,3 \%$ & 54.06 & 3.9878 & \\
\hline $1.5,1.5 \%$ & 35.4767 & 3.5747 & \\
\hline $\begin{array}{l}\text { Spinning speed } \\
\text { (krpm) and con- } \\
\text { centration }(\mathrm{m} / \mathrm{m}) \\
\text { of bis-PCBM }\end{array}$ & & & \\
\hline $3,1.5 \%$ & 21.94 & 4.0487 & 22.22 \\
\hline $2,2 \%$ & 41.85 & 10.247 & 31.993 \\
\hline
\end{tabular}

Table 9: List of measured thickness for different spin speeds, concentrations and molecular weights measured by AFM compared with unannealed values measured by $N R$.

The AFM measurements were in good agreement with the NR measurements for film thicknesses but there is some variation due to using different batches of quoted concentration which has some error every time it is reproduced, as AFM was not performed on every batch.

The impact of lateral inhomogeneities in the samples on NR curves

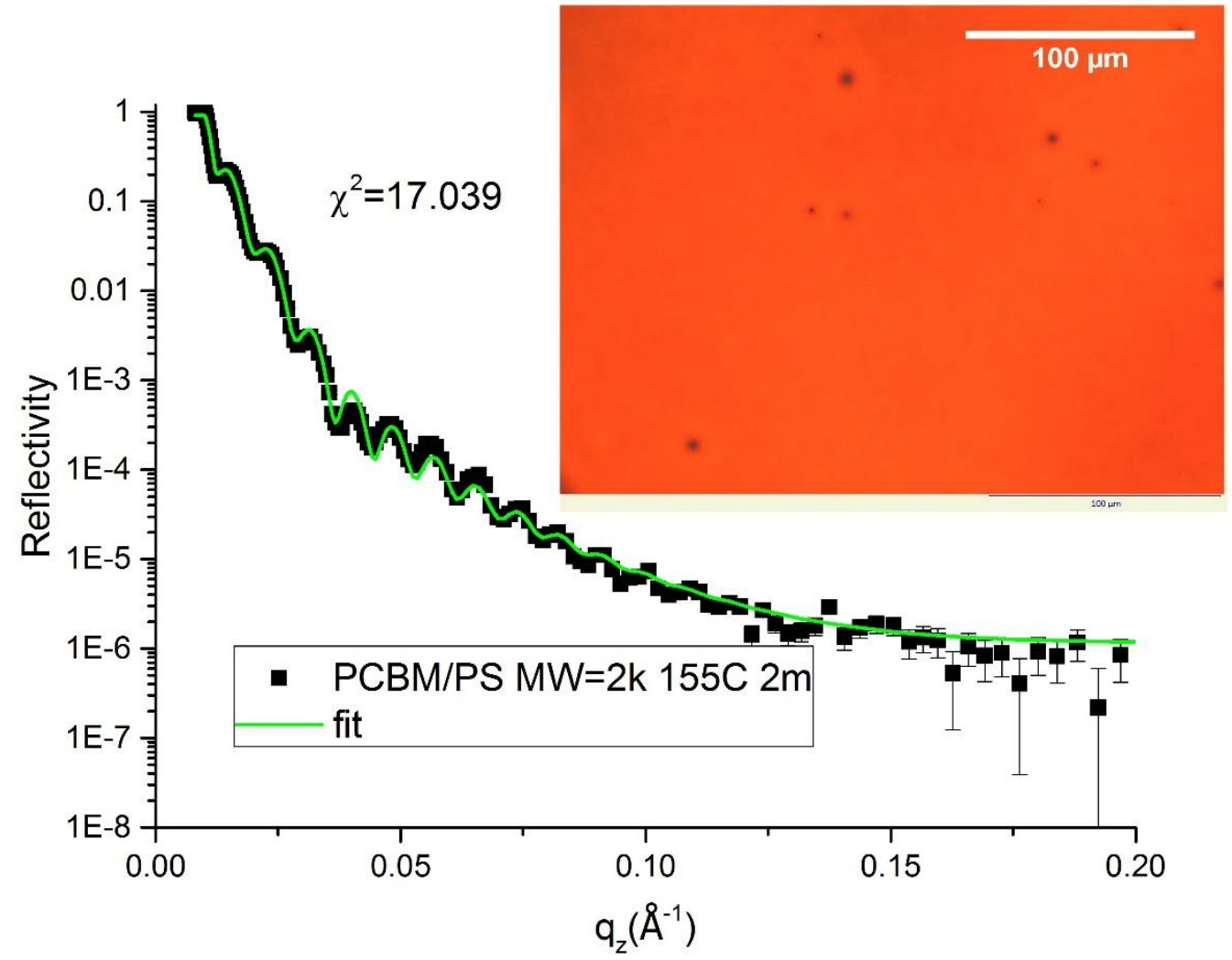

Figure 4.1-44: Micrograph and NR curve for annealed bilayer showing the lateral inhomogeneities on the sample. 
Chi-squared values are only compared for fits to data measured with the same instrumental settings and fitted using the same software. Figure 4.1-44 shows a fit with a high chi-squared and relatively poor reproduction of the fringes in the NR curve; in comparison to other $2 \mathrm{k}$ samples the $4^{\text {th }}$ fringe is too high and the $6^{\text {th }}$ and $7^{\text {th }}$ fringes are too low wrt the data). However this sample actually has a microscopically smooth surface. This sample is considered marginal, in terms of acceptability using a bilayer model. The interfacial roughness was $40.93 \AA$ and the top layer SLD was $1.61 \times 10^{-6} \AA^{-2}$. These values are included in Figure 4.1-30 and Figure 4.1-31. Figure 4.1-44 to Figure 4.1-52 show micrographs of the sample surfaces and the corresponding NR curves and fits from a range of samples in which either the fits were poor, or there were significant lateral inhomogeneities on the sample. This demonstrates that it is only in some samples that we see poor fits to the data. These figures also show some samples whose data is included in our analysis as the inhomogeneities did not seem to affect the NR curves. The samples that were not included in the final analysis for this chapter are shown in 'Rejected NR curves'.

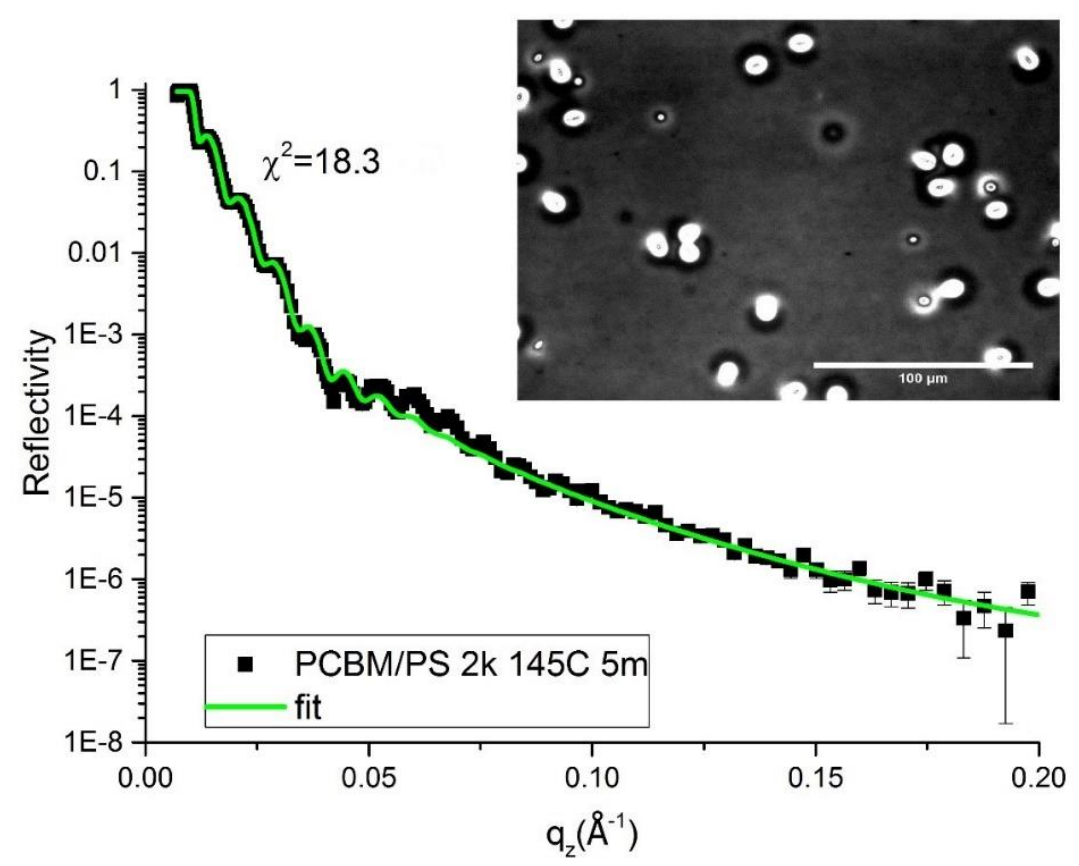

Figure 4.1-45:Optical micrograph of samples surface and NR curve and fit for the $2 k$ 145C 2 minutes sample. 


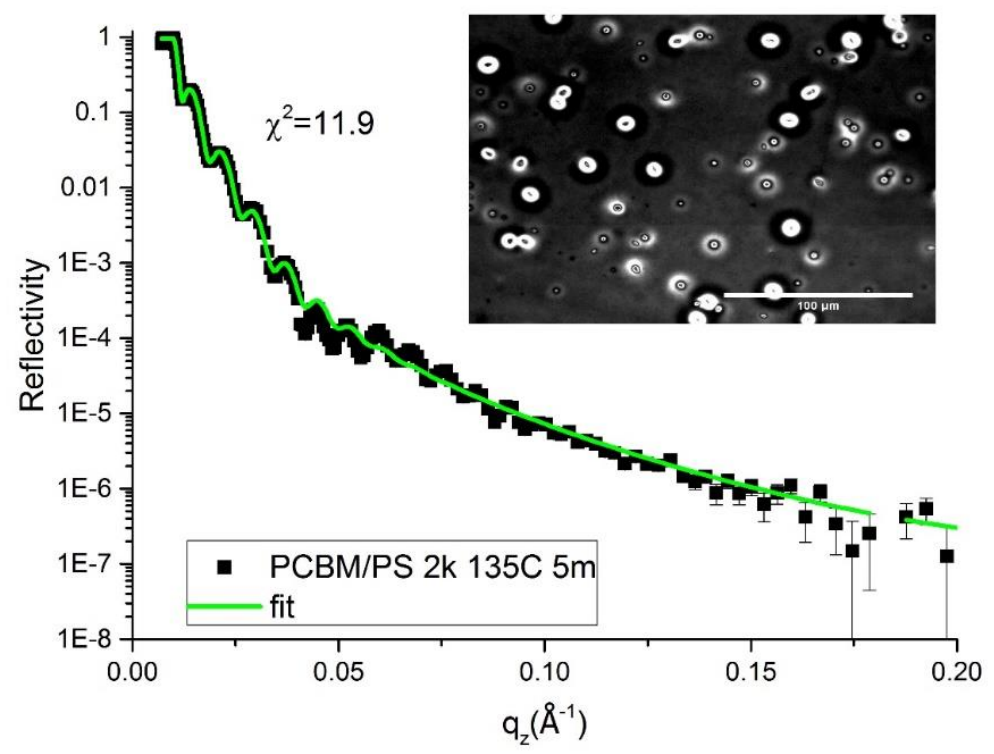

Figure 4.1-46: Optical micrograph of samples surface and NR curve and fit for the $2 k 135 C 5$ minutes sample.

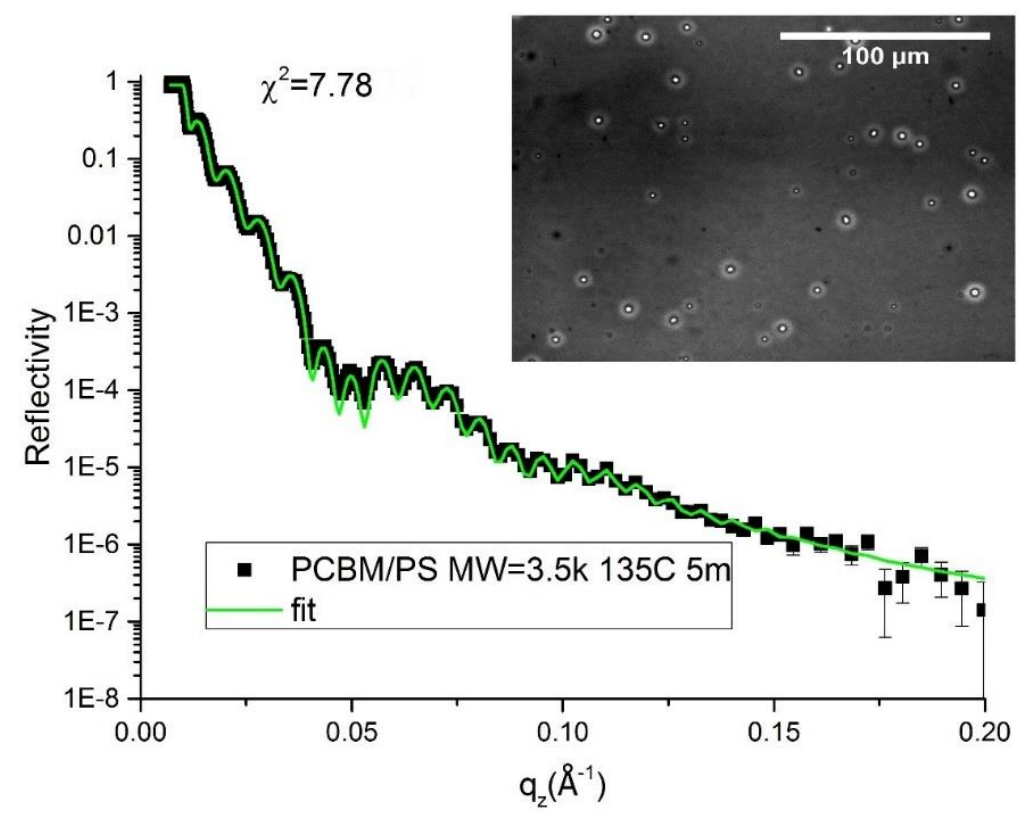

Figure 4.1-47: Micrograph and NR curve for annealed bilayer showing the lateral inhomogeneities on the sample.

Two examples of $2 \mathrm{k}$ samples with extensive lateral inhomogeneities and also with poor fits to the reflectivity curves are shown in figs $1-45$ and 1-46. A further $2 \mathrm{k}$ sample, annealed at a temperature of $140{ }^{\circ} \mathrm{C}$ had a very similar level of lateral inhomogeneities and a similarly poor fit to the fringes. As discussed previously, these three samples are not included in the final analysis of interfacial width and layer 
SLD. However, not all samples containing lateral inhomogeneities resulted in poor fits to the reflectivity. Figure 4.1-47 shows a relatively good fit to a $3.5 \mathrm{k}$ sample, with good reproduction of the fringes, despite the appearance of microscopic defects on the sample surface. This shows that in some cases good bilayer fits can be obtained despite the presence of lateral inhomogeneities, that do not appear to significantly impact the NR curves. Figure 4.1-48 and Figure 4.1-49 show AFM image and height profiles of defects from the sample shown in Figure 1-47. The defects protrude to varying degrees from the film surface and some are anisotropic and resemble initial stages of needle-like micron-sized crystals. These defects are typical examples and the defects present in other samples have similar features.

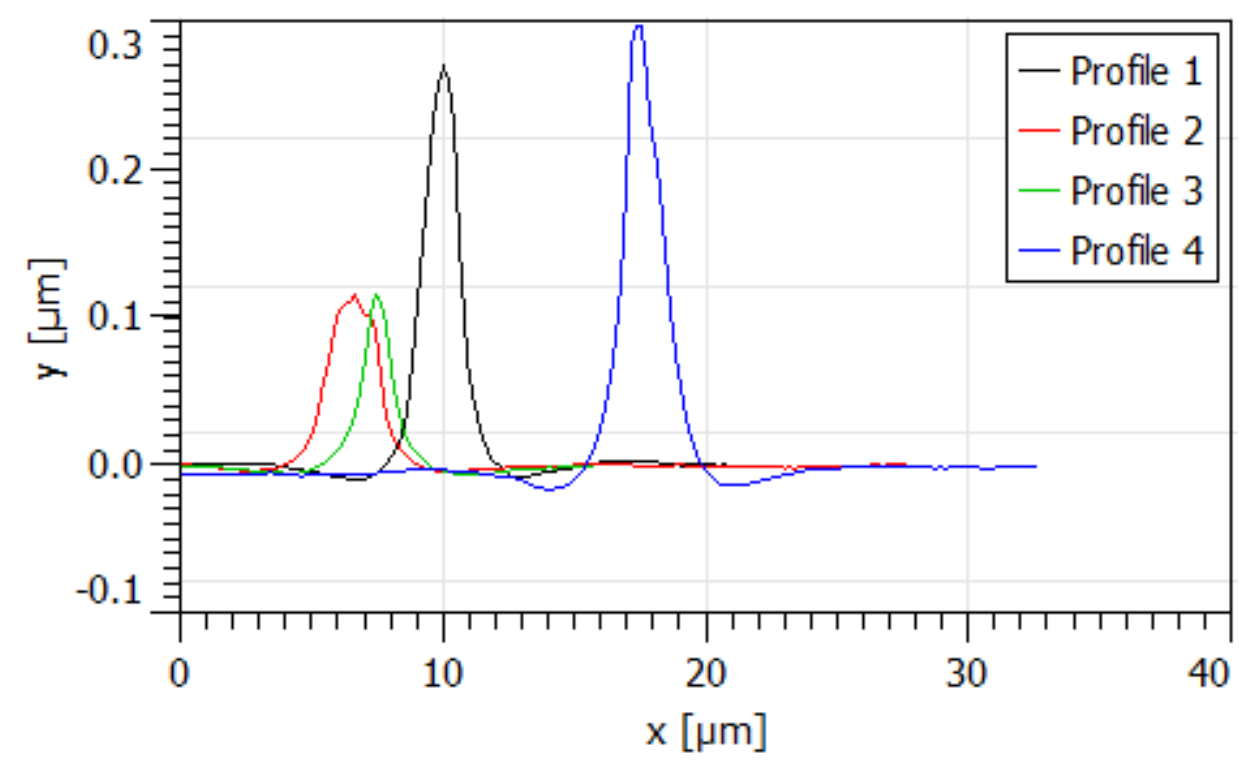

Figure 4.1-48: Defect height profiles for lines from Figure 4.1-49. 


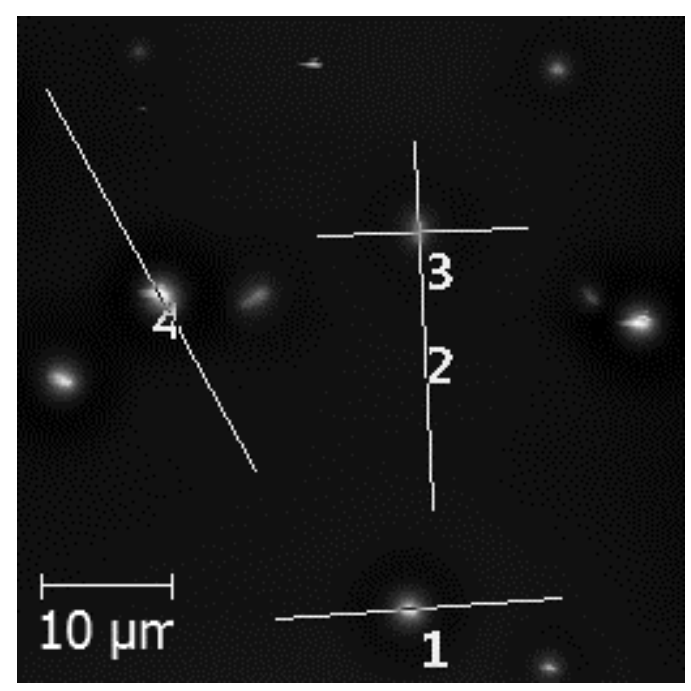

Figure 4.1-49: AFM of defects from 3.5k 135C 5m sample

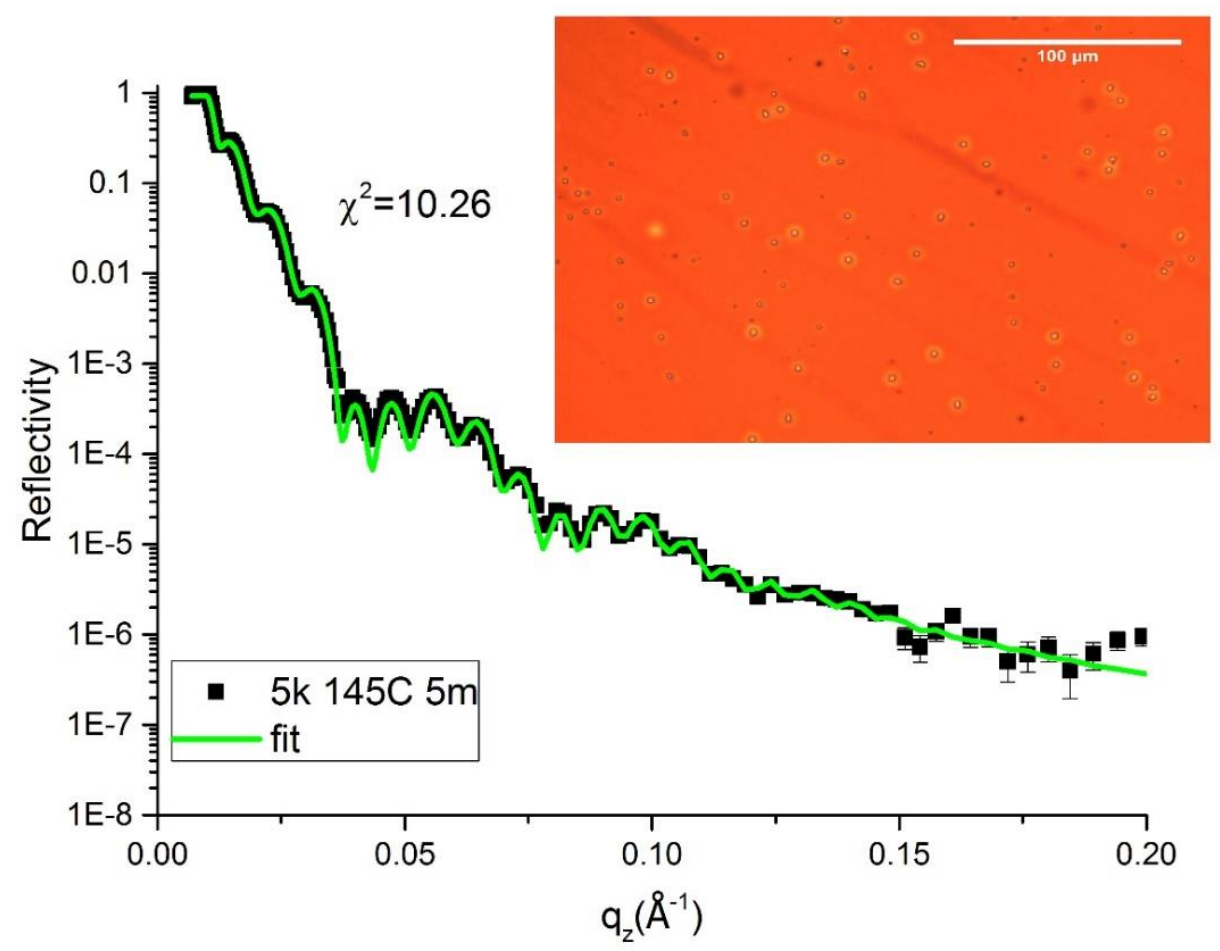

Figure 4.1-50: Micrograph and NR curve for annealed bilayer showing the lateral inhomogeneities on the sample. 


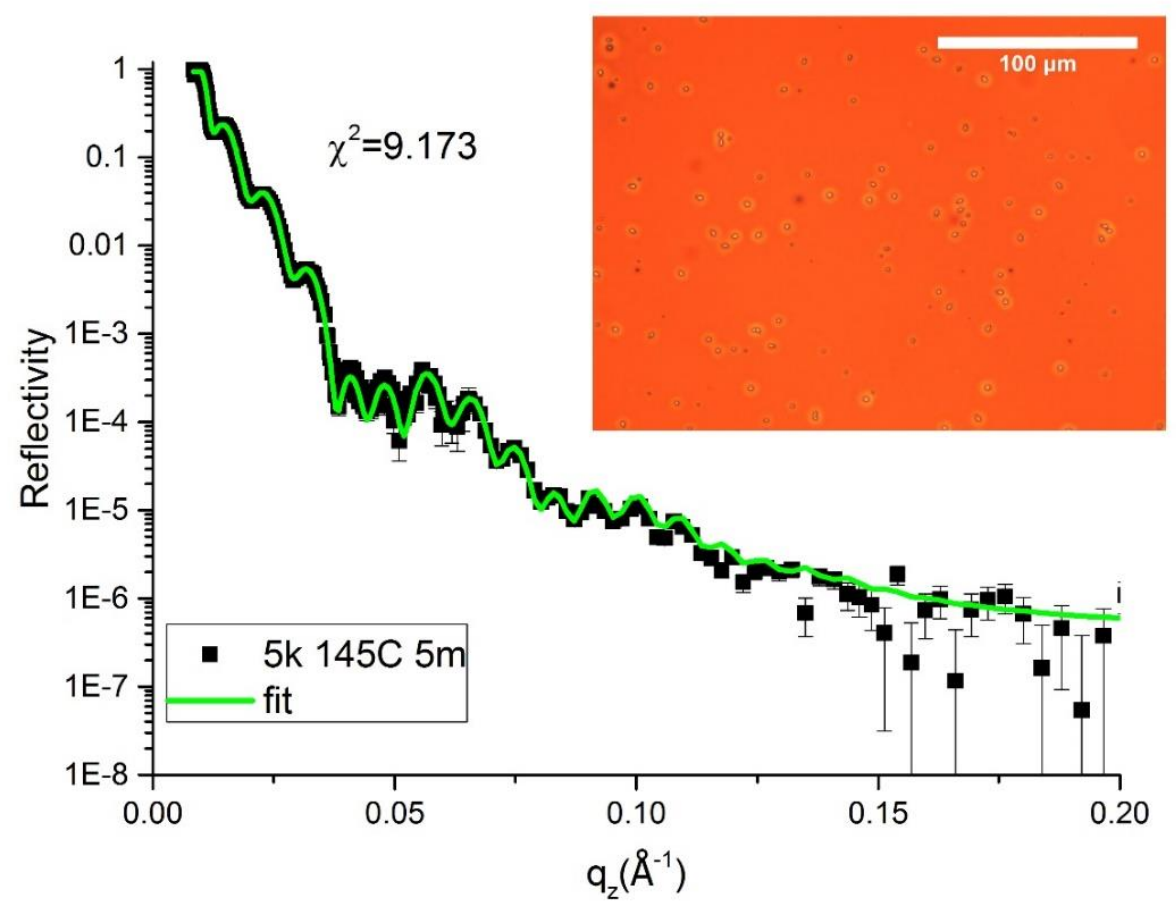

Figure 4.1-51: Micrograph and NR curve for annealed bilayer showing the lateral inhomogeneities on the sample.

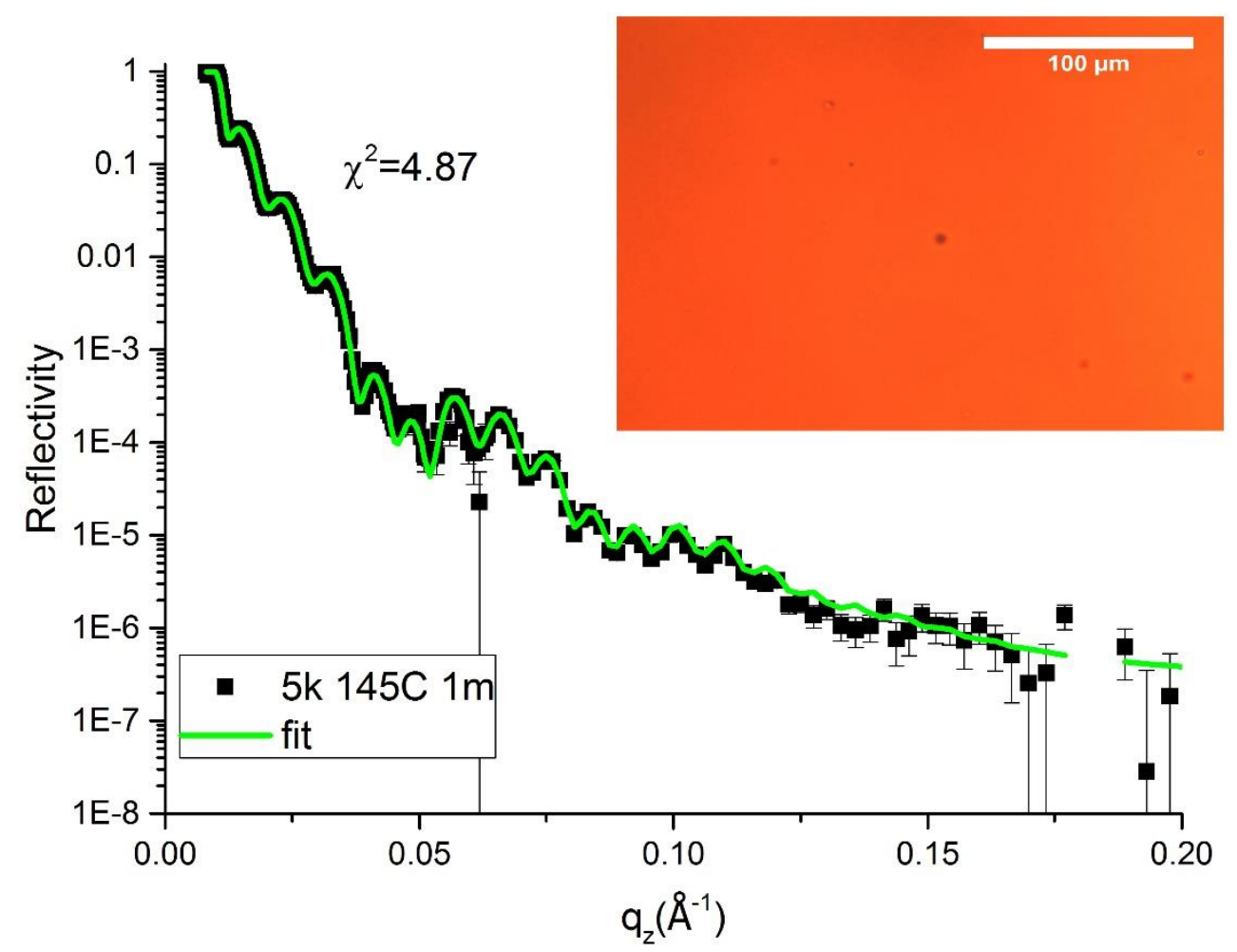

Figure 4.1-52: Micrograph and NR curve for annealed bilayer showing the lateral inhomogeneities on the sample. 
Figure 4.1-50 to Figure 4.1-52 show some examples of varying degrees of lateral inhomogeneities on a selection of $5 \mathrm{k}$ samples. These images and corresponding NR curves show that for all of these samples regardless of the density of inhomogeneities there is a consistent behaviour. The $5 \mathrm{k}$ samples have a lower interfacial roughness than all of the $2 \mathrm{k}$ samples for all of the samples of acceptable quality as in Fig 4.1-30, irrespective of the level of lateral inhomogeneity on the samples. They also have a higher interfacial roughness than the 20k samples in the majority. For this reason, and after careful examination of some of the $2 \mathrm{k}$ samples, and subsequent rejection of a small number of samples, there is no evidence to suggest that the results shown in Figure 4.1-30 are influenced by lateral inhomogeneities on the samples. Several $2 \mathrm{k}$ samples showing no evidence of significant lateral inhomogeneities at all (such as the sample shown in Figure 1-55, and several samples annealed at $120^{\circ} \mathrm{C}$ and $125^{\circ} \mathrm{C}$ ), have interfacial roughness that are significantly higher than all of the $5 \mathrm{k}$ samples. The roughness measured by NR does not seem to be affected by the presence of dots until the level (a combination of the density and amplitude of the inhomogeneities) reaches some critical value.

Figure 4.1-53 shows a smooth surface from AFM and a GIXD detector map, both indicating the absence of significant crystallisation. The absence of sharp Bragg peaks in the GIXD map indicates that there is no planar ordering within these samples indicating (at least mostly) amorphous material. This sample constitutes the upper end of the sample quality spectrum. Figure 4.1-53 and Figure 4.1-46 represent the full range of densities of lateral homogeneities found on the $2 \mathrm{k}$ samples. 

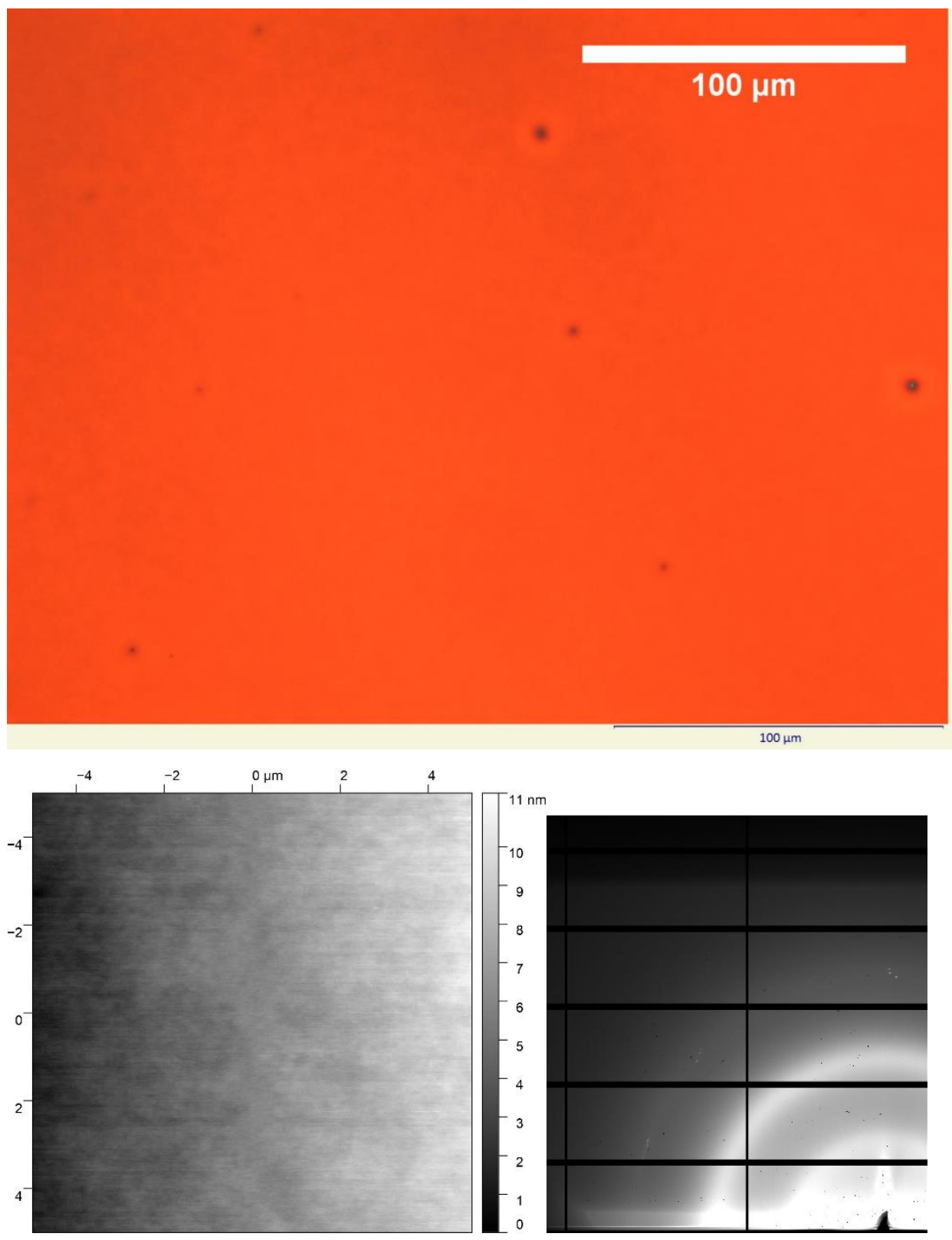

Figure 4.1-53: Top: micrograph of PCBM/PS $M w=2 k$ Da sample annealed at $140 C$ $1 \mathrm{~m}$, bottom left, AFM image; bottom right: GIXD image. The rms roughness from AFM for this sample is $2.36 \mathrm{~nm}$.

The $2 \mathrm{k}$ samples for which the interface roughness and top layer SLD are plotted in Figure 4.1-30 and Figure 4.1-31 (which excludes the 4 rejected samples in section 1.7.6) show a density of lateral inhomogeneities that is either similar to or significantly lower than that for the $5 \mathrm{k}$ samples. This means that the level of lateral inhomogeneities is not influencing the values of the fit parameters and does not account for seeing larger interfacial roughness in the $2 \mathrm{k}$ samples than the $5 \mathrm{k}$ samples. 

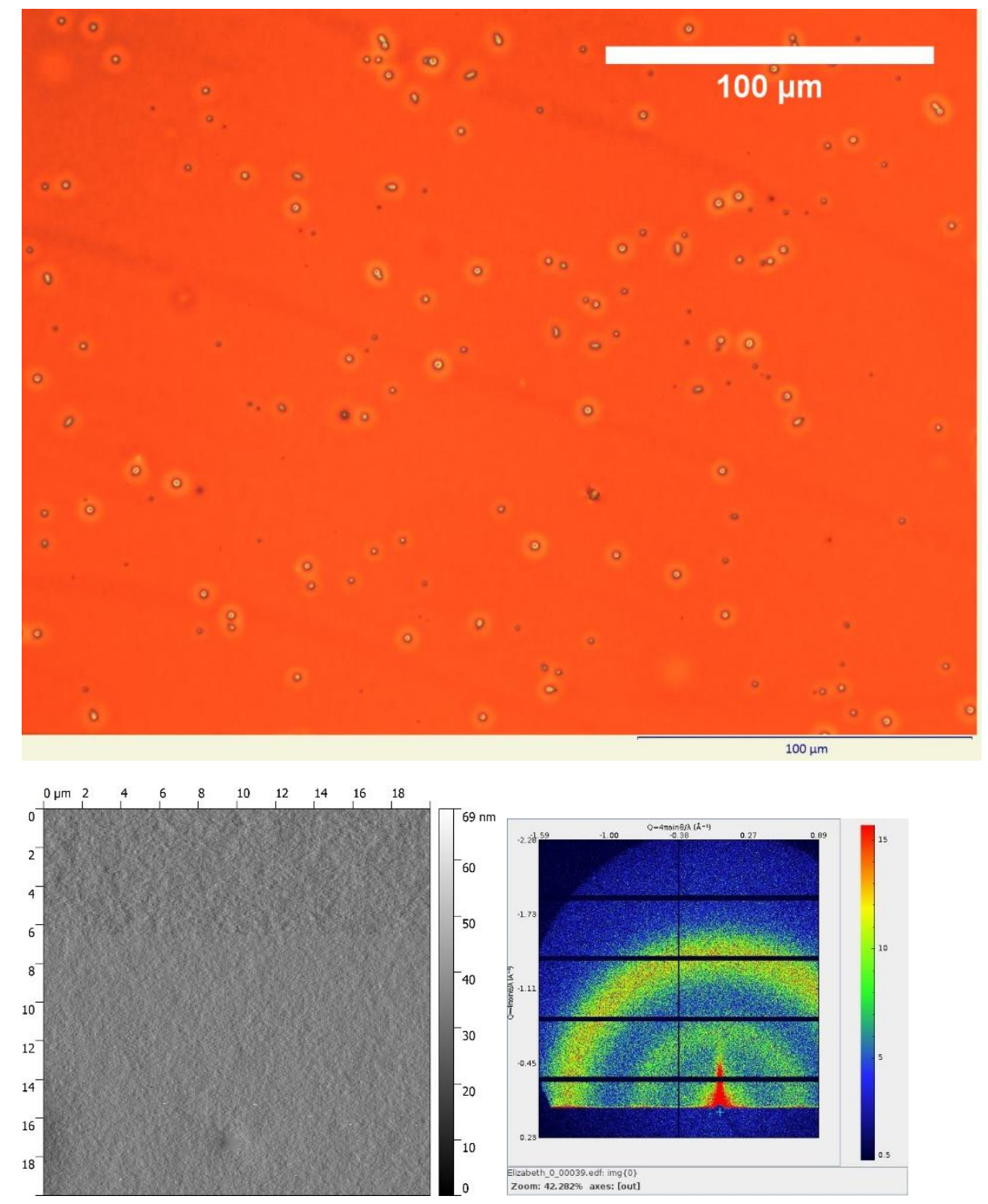

Figure 4.1-54: Top: micrograph of PCBM/PS Mw=5k Da sample annealed at $145 C$ $5 \mathrm{~m}$, bottom left, AFM image from a region between the lateral inhomogeneities; bottom right: GIXD image. Rms roughness measured by AFM is 5.5nm. This is the same sample as in Figure 4.1-51.

Figure 4.1-54 shows data for a typical 5k sample with a moderate level of lateral inhomogeneities. The GIXD map for this sample indicates that there is not a significant level of crystallisation. This and the well-fitted NR curves for such samples, suggests that the fitted parameters for samples with this level of lateral inhomogeneities represent the composition profile between two coexisting (amorphous liquid) compositions, in the regions between the lateral inhomogeneities. 


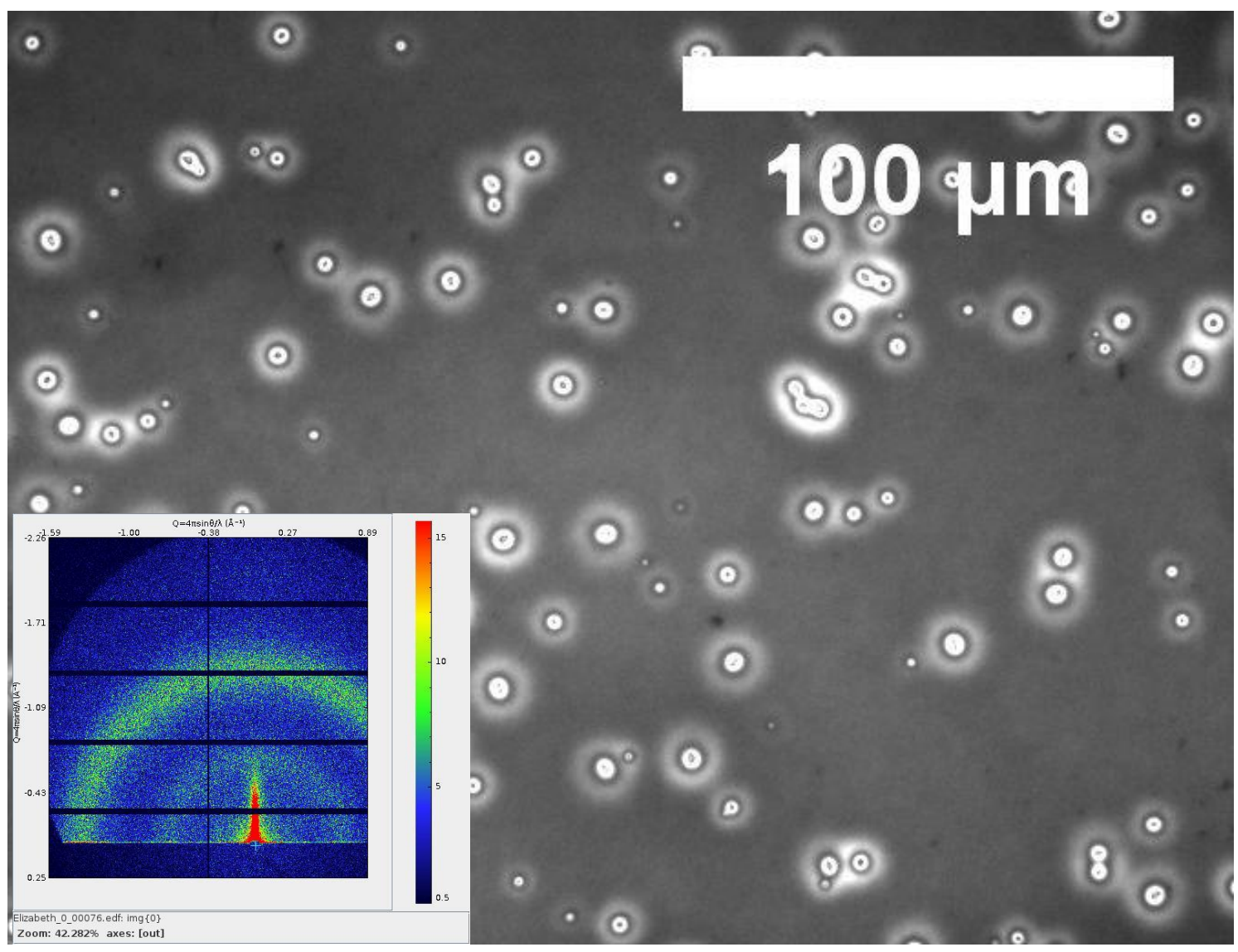

Figure 4.1-55: PCBM/PS MW=2k Da sample annealed in-situ over a range of temperatures. Images taken after cooling.

Figure 4.1-55 shows a micrograph and a GIXD map for the in-situ 2k sample, indicating that even annealing for an extended period of time did not significantly affect the amorphous rings. No evidence of crystallisation is seen. This sample had considerably washed out fringes, suggesting that there may be deviations from the average thickness of each layer causing overlapping of the scattered beam from regions of different thicknesses. The parameters for this sample, after annealing and cooling, are not included in Figure 4.1-30 and Figure 4.1-31. 


\section{Rejected NR curves}

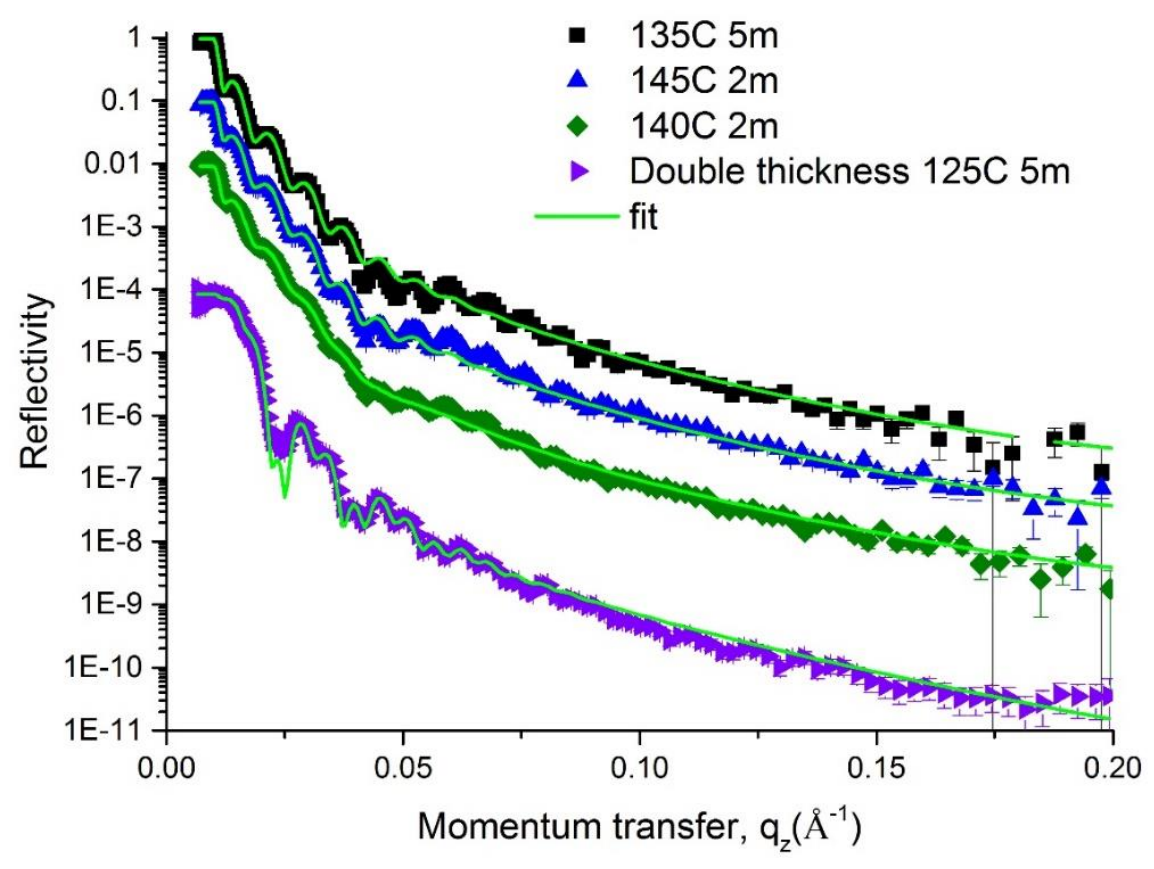

Figure 4.1-56: $P C B M / P S M W=2 k$ Da bilayers whose data we have excluded from our analysis. The data plotted here from the samples annealed at $135^{\circ}, 140{ }^{\circ}$ and 145 ${ }^{\circ} \mathrm{C}$ are not shown in the main body of this chapter, but the double thickness sample data and fit has already been plotted in Figure 4.1-15. Optical micrographs for the $135^{\circ} \mathrm{C}$ and $145^{\circ} \mathrm{C}$ samples are shown in figures 4.1-45 and 4.1-46.

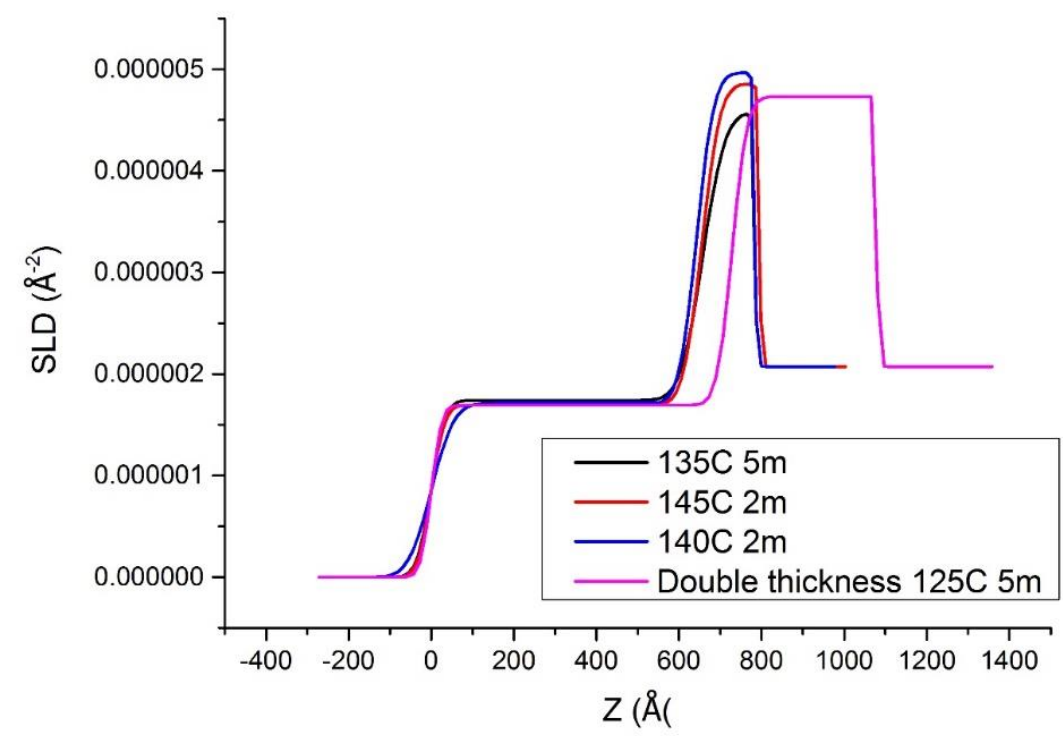

Figure 4.1-57: $P C B M / P S$ bilayers with $M W=2 k$ Da SLD profiles for rejected data due to a fit that didn't capture the fringes of the data. The samples annealed at 135, 140 and 145 are not shown in the main body of this chapter, but the double thickness sample SLD profile has already been plotted in figure 4.1-15. 
The data from the samples shown in Figure 4.1-56 and Figure 4.1-57 is not included in the analysis in Figure 4.1-30 and Figure 4.1-31. As can be seen in Figure 4.1-56 the fringes in the fits to the $135^{\circ} \mathrm{C} 5 \mathrm{~m}, 140^{\circ} \mathrm{C} 2 \mathrm{~m}$ and $145^{\circ} \mathrm{C} 2 \mathrm{~m}$ samples are quite 'washed out' and do not match the data well. It is likely that this is due to the presence of lateral inhomogeneities that cause a bilayer fit, with Gaussian interface roughness, to be less physically reasonable (the amplitude of the fringes in these three curves, is actually lower than for the $2 \mathrm{k}$ samples in Figure 4.1-2). The attempt to use a bilayer model to fit this data smears out the SLD profile (which is laterallyaveraged over a distance of order tens of microns), effectively increasing the interface roughness in the fit. The values that are returned for the interfacial roughness fit parameter for the $135^{\circ} \mathrm{C} 5 \mathrm{~m}, 140^{\circ} \mathrm{C} 2 \mathrm{~m}$ and $145^{\circ} \mathrm{C} 2 \mathrm{~m}$ samples are 60.1 A, $47.2 \AA$ and $49.9 \AA$ respectively.

While we did not include the double (PCBM layer) thickness 2k 125C 5m (Figure 4.1-56), interface roughness fit parameter in our final PCBM/PS roughness/SLD v MW plots (as a high density of inhomogeneities covered over half of the sample), the SLD profile from this sample was very similar to that of the other double thickness sample (annealed at $120{ }^{\circ} \mathrm{C}$; this sample showed a low density of lateral inhomogeneities); the SLDs of the two layers were very similar and the only significant difference was an increased interface roughness of 40A cf 27A. Both double thickness samples are shown in Figure 4.1-15 and Figure 4.1-16. 


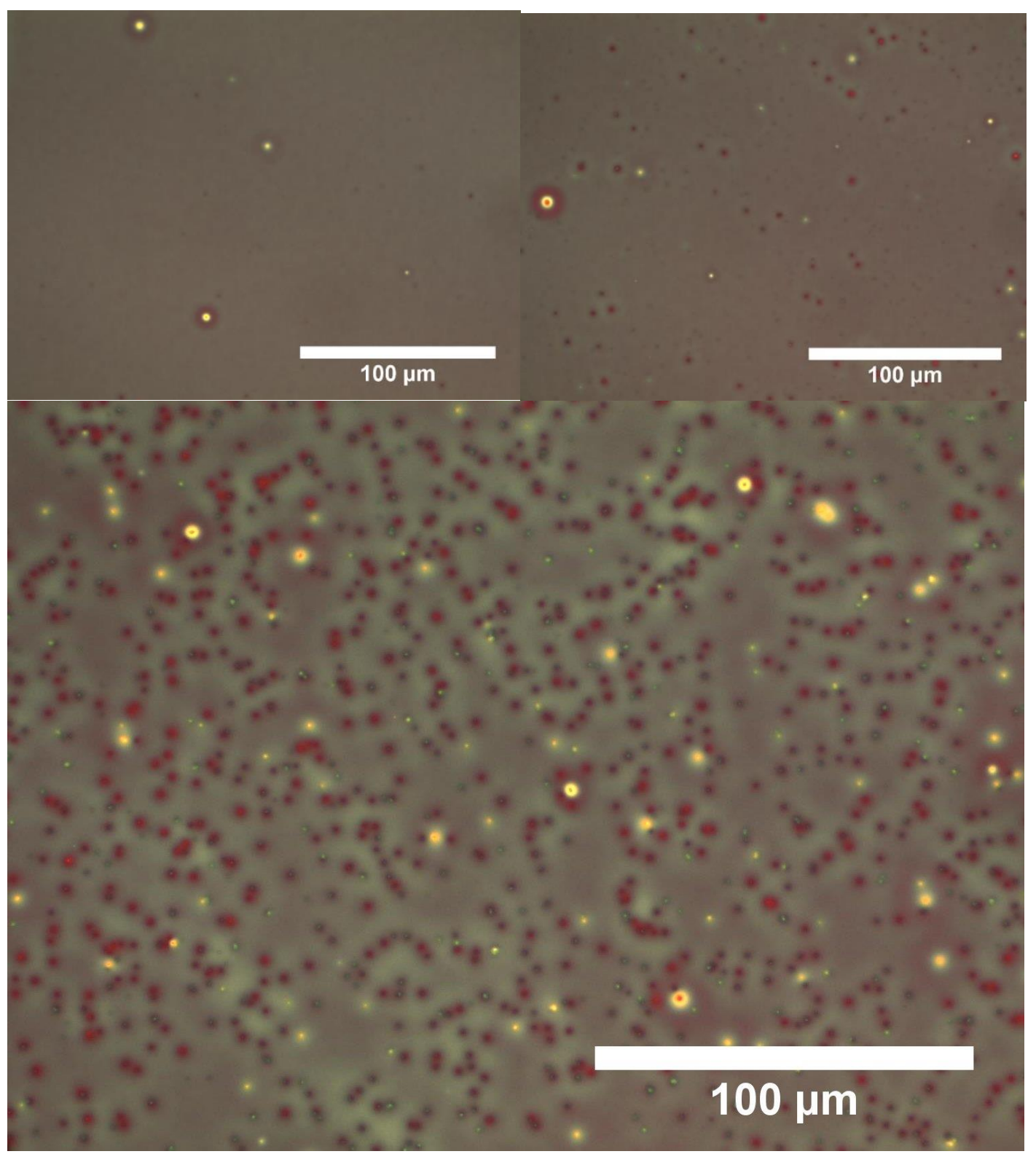

Figure 4.1-58: Micrographs for PCBM/PS 2k double (PCBM layer) thickness 125C $5 \mathrm{~m}$ sample. Top left: a smooth (uniform) area, top right: intermediate area, bottom: the significant presence of inhomogeneities. Out of 30 images chosen from different locations across the entire sample (chosen in an unbiased way by moving the microscope stage without looking at the image), 17 were covered inhomogeneities similar to the level shown in the bottom image, 5 were smooth (uniform), and 8 were intermediate). 


\section{Testing the robustness of the fitted parameters: top layer SLD and intefacial roughness}

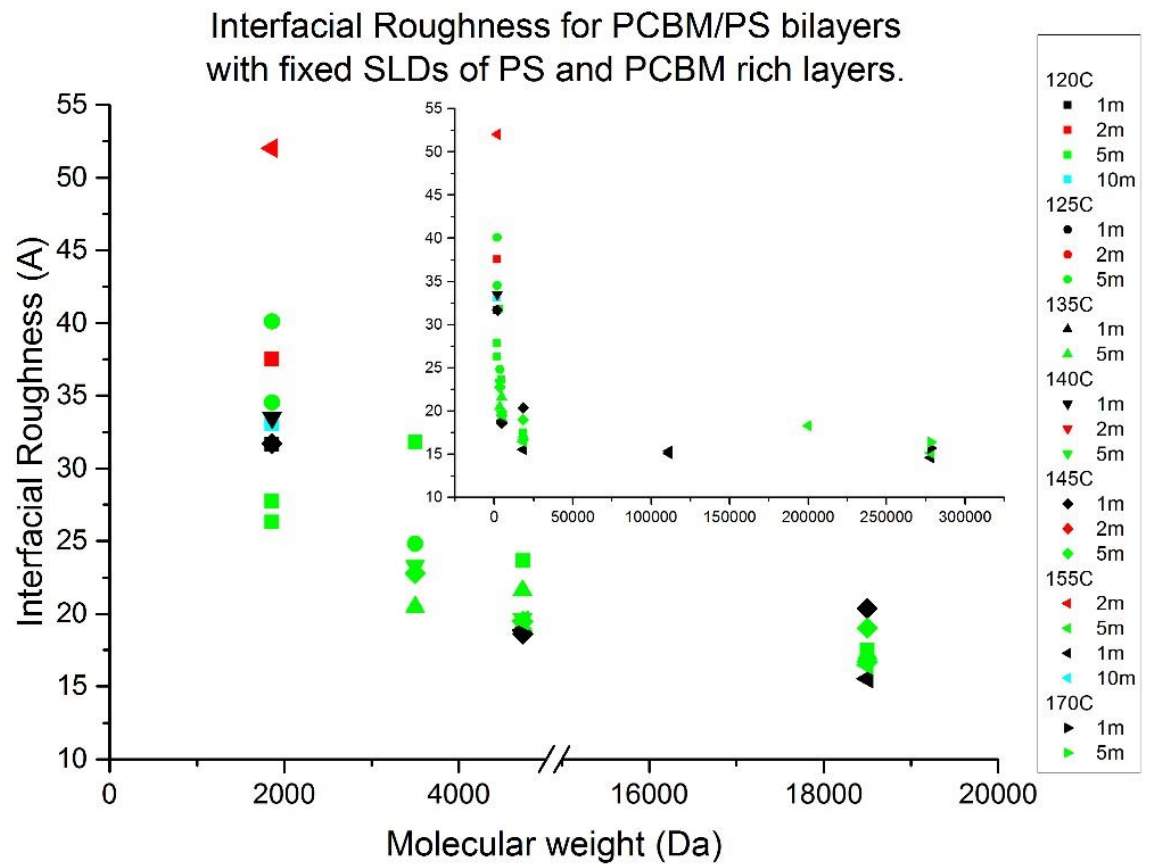

Figure 4.1-59: Interfacial roughness for PCBM/PS bilayers fitted with a constant average $S L D$ of top layer, $1.65 \times 10^{\wedge}-6$, and bottom layer, $4.65 \times 10^{\wedge}-6$.

Figure 4.1-59 shows the interfacial roughness values fitted to the data, but with fixed top and bottom layer SLDs of $1.65 \times 10^{-6} \AA^{-2}$ and $4.65 \times 10^{-6} \AA^{-2}$ respectively. These are the average values for all the measurements in this system. Figure 4.1-59 shows that when the number of adjustable parameters is reduced from 6 to 4 in this way, a very similar plot for interface roughness versus MW (in comparison to Figure 4.1$30)$ is obtained.

\section{Accounting for fractional coverage by PS}

During sample fabrication for the Figaro samples, the top layer sometimes cracked during floating leading to samples looking like Figure 4.1-60. This affected the reflectivity profiles in some samples where the cracked exposed surface area of the PCBM was greater than $5 \%$. 


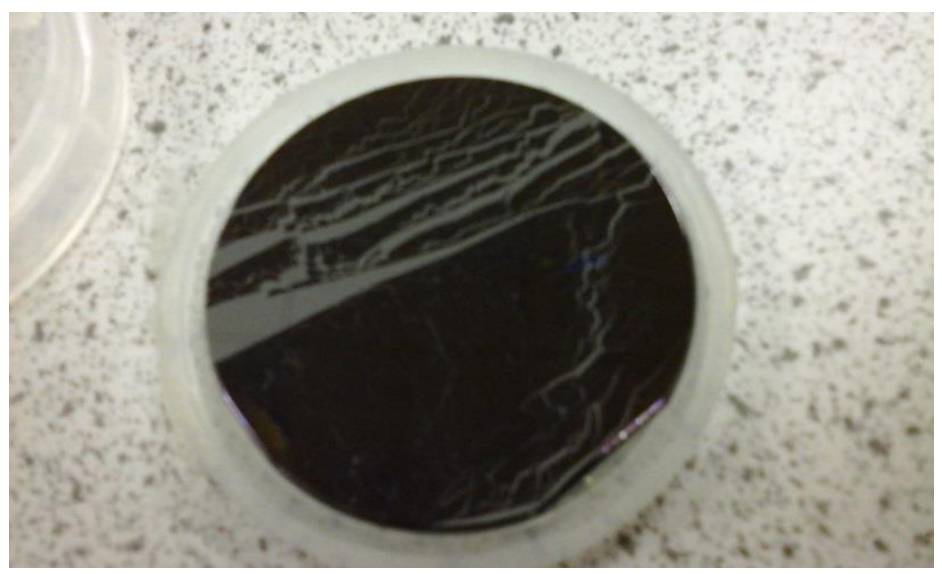

Figure 4.1-60: Image of a sample with a cracked top PS layer.

The sample coverage of PS was measured using ImageJ which takes the threshold of the colours present in a selected area to calculate the values presented in Table 10

\begin{tabular}{ll}
\hline \hline Sample & \% coverage by PS \\
\hline $2 \mathrm{k} 145^{\circ} \mathrm{C} 1 \mathrm{~m}$ & 99.9 \\
$2 \mathrm{k} 140^{\circ} \mathrm{C} 1 \mathrm{~m}$ & 99.3 \\
$2 \mathrm{k} 145^{\circ} \mathrm{C} 1 \mathrm{~m}$ & 98.2 \\
$2 \mathrm{k} 155^{\circ} \mathrm{C} 1 \mathrm{~m}$ & 82.3 \\
$2 \mathrm{k} 155^{\circ} \mathrm{C} 2 \mathrm{~m}$ & 98.7 \\
$2 \mathrm{k} 145^{\circ} \mathrm{C} 1 \mathrm{~m}$ & 94.2 \\
$5 \mathrm{k} \mathrm{unannealed}$ & 97.9 \\
$5 \mathrm{k} 145^{\circ} \mathrm{C} 5 \mathrm{~m}$ & 98.7 \\
$5 \mathrm{k} 155^{\circ} \mathrm{C} 5 \mathrm{~m}$ & 87.1 \\
$5 \mathrm{k} 145^{\circ} \mathrm{C} 1 \mathrm{~m}$ & 99.8 \\
$5 \mathrm{k} 155^{\circ} \mathrm{C} 5 \mathrm{~m}$ & 96.5 \\
$20 \mathrm{k} 145^{\circ} \mathrm{C} 1 \mathrm{~m}$ & 96 \\
$20 \mathrm{k} 145^{\circ} \mathrm{C} 5 \mathrm{~m}$ & 92.4 \\
$20 \mathrm{k} 155^{\circ} \mathrm{C} 1 \mathrm{~m}$ & 97 \\
$20 \mathrm{k} 155^{\circ} \mathrm{C} 5 \mathrm{~m}$ & 93.5 \\
$100 \mathrm{k} \mathrm{unannealed}$ & 98.3 \\
$100 \mathrm{k} 155^{\circ} \mathrm{C} 1 \mathrm{~m}$ & 99.9 \\
$100 \mathrm{k} 155^{\circ} \mathrm{C} 5 \mathrm{~m}$ & 96.6 \\
$300 \mathrm{k} 155^{\circ} \mathrm{C} 1 \mathrm{~m}$ & 97.1 \\
$300 \mathrm{k} 155^{\circ} \mathrm{C} 5 \mathrm{~m}$ & 97.5 \\
$300 \mathrm{k} 170^{\circ} \mathrm{C} 1 \mathrm{~m}$ & 99.9 \\
$300 \mathrm{k} 170^{\circ} \mathrm{C} 5 \mathrm{~m}$ & 98.7 \\
\hline
\end{tabular}

Table 10: Values obtained from ImageJ of the coverage of the top PS layer on the relevant samples.

To account for this incomplete coverage we subtracted an area-weighted fraction of the reflectivity from a 20nm PCBM single layer from the total reflectivity and refitted this data. This was carried out for those samples (4 in total) with less than $94 \%$ coverage (this was not necessary for the other bilayers as the reflectivity from the 
PCBM/silicon only areas was negligible for PS coverages greater than this). These samples had exhibited high chi-squared values but after the subtraction had values similar to the other samples, and gave improved fits (see Figure 4.1-61). Originlab software was used to subtract the raw reflectivity of PCBM from the bilayer data (and then refitted); a linear interpolation was used whenever the q-values differed.
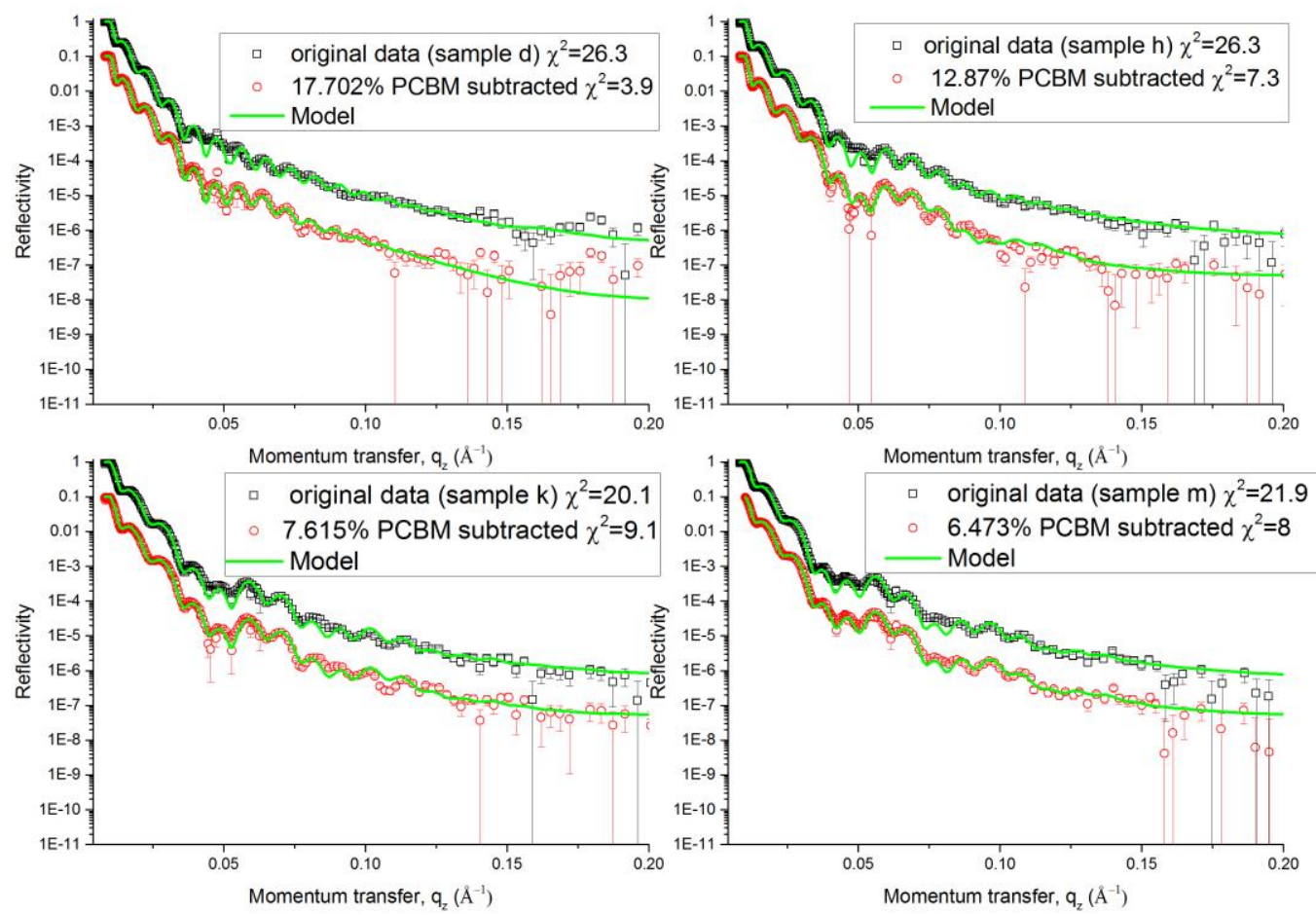

Figure 4.1-61: This figure shows the reflectivity before and after subtraction of the reflectivity from a PCBM single layer for 4 sample $d, h, k$ and $m$. These samples were ex-situ annealed for 1 min at $155^{\circ} \mathrm{C}(2 k$-PS sample $d), 1$ min at $155^{\circ} \mathrm{C}(5 k-P S$ sample h), 5 mins at $145^{\circ} \mathrm{C}(20 \mathrm{k}-\mathrm{PS}$ sample $k)$ and 5 mins at $155^{\circ} \mathrm{C}(20 \mathrm{k}-\mathrm{PS}$ sample $m)$.The subtraction was performed using the percentage PCBM-only area coverage on each sample and the reflectivity data from a PCBM single layer sample of the same thickness. In all four cases the chi-squared was drastically improved. 


\subsection{The impact of molecular weight on mixing and interfacial width in bis-PCBM/PS bilayers}

In this chapter, the results from NR experiments on bis-PCBM/PS bilayers are presented. bis-PCBM is another candidate for acting as an electron acceptor in OPVs. This molecule has a extra side-chain, compared to PCBM. Crystallisation is inhibited in bis-PCBM, possibly due to the lack of control of the relative position and orientation of the two side-chains. In terms of miscibility with PS, we expect the extra side chain to reduce the chi-parameter. This is because of the attractive effect of van der Waals forces between chemically similar materials. The $\mathrm{C}_{12} \mathrm{H}_{14} \mathrm{O}_{2}$ side chain is therefore expected to have a higher compatibility with PS $\left(\left(\mathrm{C}_{8} \mathrm{H}_{8}\right) n\right)$, than does the C60 part of the molecule.

This chapter will discuss results from thermally annealed bis-PCBM/PS bilayers to find out if the behaviour is comparable to the PCBM/PS bilayers in terms of the effect of molecular weight on interfacial roughness and SLD values of the layers. Ex-situ and in-situ data are presented and discussed.

\subsubsection{Bilayer fits for $2 \mathrm{k}, 3.5 \mathrm{k}, 5 \mathrm{k}, 20 \mathrm{k}$ and $100 \mathrm{k}$ PS ex-situ samples} Figure 4.2-1 and Figure 4.2-2 show the fits and SLD profiles for a range of molecular weights of PS in bis-PCBM/PS bilayers; 2k, 3.5k, 5k, 20k, and 100k nominally. The unannealed sample has a significantly different composition profile from the annealed samples in terms of the interfacial roughness and the SLD of the top layer. There is a more distinctive difference post-annealing in fitted parameters than was seen for the PCBM/PS samples. These are bilayer fits fitted in the same way as for the PCBM/PS samples, and using a fixed oxide layer. The unannealed $2 \mathrm{k}$ sample has PS layer thickness of 58.3nm, PS layer SLD of $1.21 \times 10^{-6} \AA^{-2}$, top layer roughness of $13.9 \AA$, bis-PCBM layer thickness of $31.9 \mathrm{~nm}$, bis-PCBM SLD of 3.71 $\mathrm{x} 10^{-6} \AA^{-2}$ and interfacial roughness of $0.002 \AA$. As with the PCBM/PS system, the bilayer model reveals significant diffusion of the bis-PCBM (originating in the bottom layer) into the PS (top) layer, increasing the thickness and SLD of the top layer. However, unlike PCBM/PS, the scale of this mass transfer is larger than in PCBM/PS, with a larger increase in the top layer SLD and near depletion of the 
bottom layer. In fact, the size of the interfacial roughness fitted by a bilayer model approaches the thickness of the bottom layer.
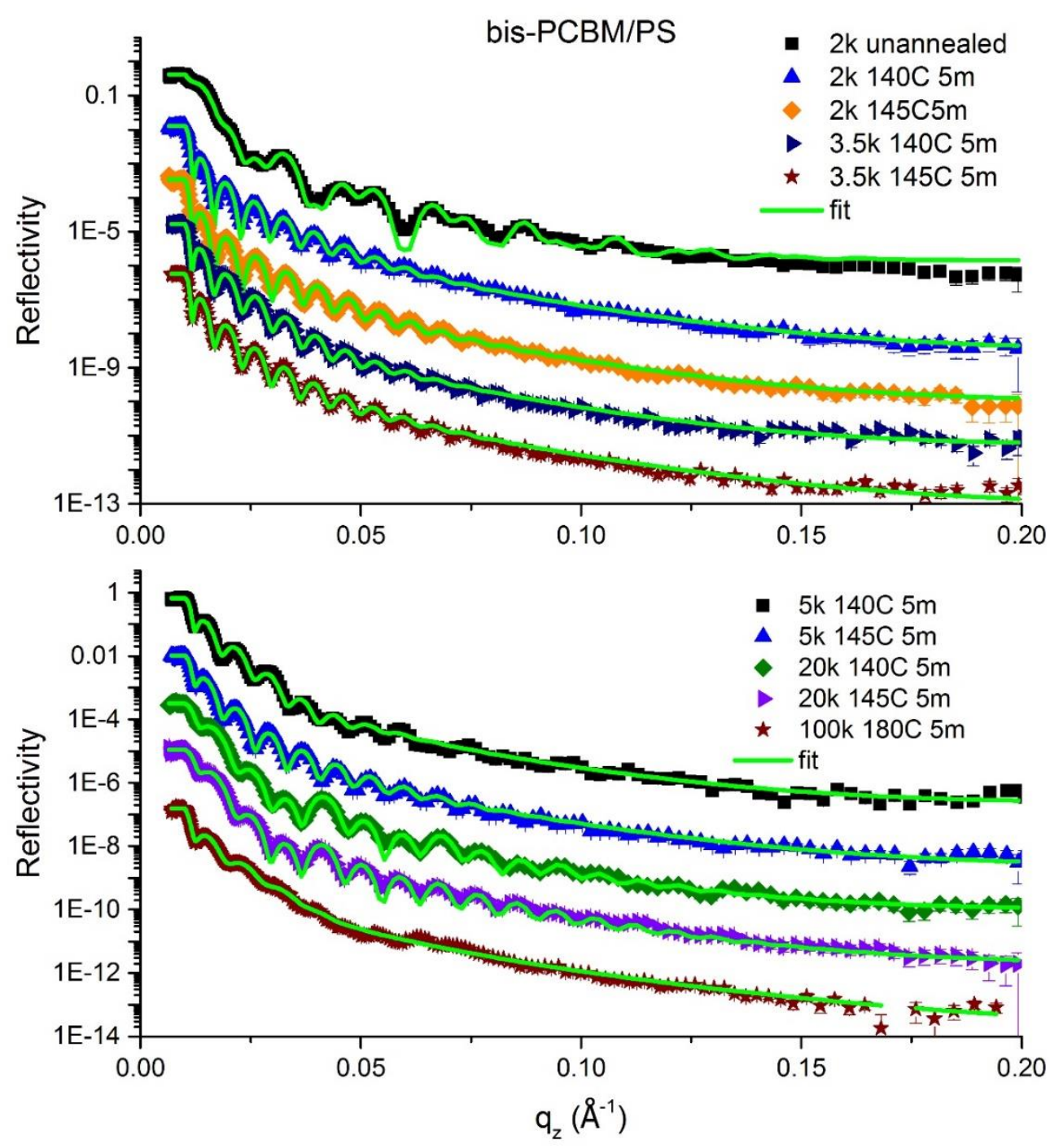

Figure 4.2-1: NR curves and fits for bis-PCBM/PS bilayers of various PS MW. This graph shows data fitted with a bilayer model.

Some of the fits in Figure 4.2-1 do not completely capture the contours of the NR curves. The $5 \mathrm{k} 140{ }^{\circ} \mathrm{C}$ sample and the $100 \mathrm{k}$ sample have features that are not reproduced in the fits. As can be seen from the SLD profiles, fitting a bilayer to these curves leads to very large interfacial roughness leaving very little pure material in the bottom layer. In the $2 \mathrm{k}$ and $3.5 \mathrm{k}$ fits the question of whether a single layer fit 


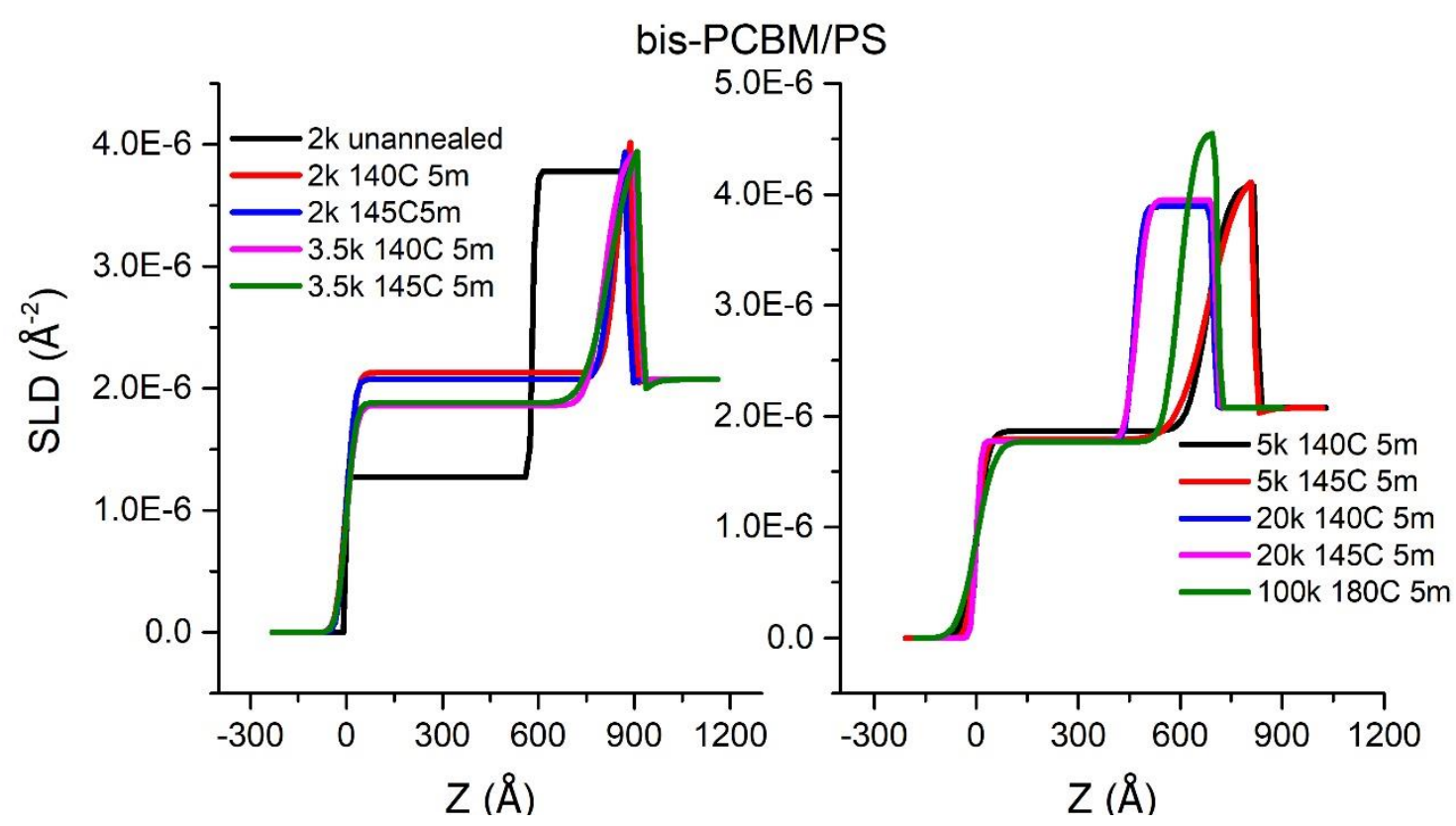

Figure 4.2-2: SLD profiles for bis-PCBM/PS bilayers of various PS MW and unannealed or annealed at various times using a bilayer model.

would give better results arises, as the fitted bottom layer is extremely thin and in the NR curves there appears to be only one periodicity. The 20k PS bilayers seem to have a reasonable amount of pure bottom layer (fabricated as pure bis-PCBM) left after annealing suggesting that modelling these samples with Gaussian roughness between two uniform layers is acceptable. For the other samples, it must be taken into consideration that the bottom layer may have become depleted before equilibrium composition was reached. If this is the case, then the error function fitted to the interface by theory will be inadequate to capture the gradient of the composition. For this reason, fitting multiple thinner layers to the data will provide insight into a more accurate composition profile.

\subsubsection{Single layer fits to the $2 \mathrm{kPS}$ bilayers}

As mentioned previously it was observed that the $2 \mathrm{k}$ PS samples exhibited a single periodicity by eye and so a single layer fit was performed on these samples. The chisquared value for these fits was considerably higher than the bilayer fits and also the multilayer fits shown in section 4.2.3 below. Figure 4.2-3 shows these fits and it is obvious that they do not capture the fringes well at all, demonstrating clear evidence for the existence of more than one layer within these samples. These fits were therefore rejected and only SLD profiles and interfacial roughness values from bilayer and multilayer fits will be compared below. 


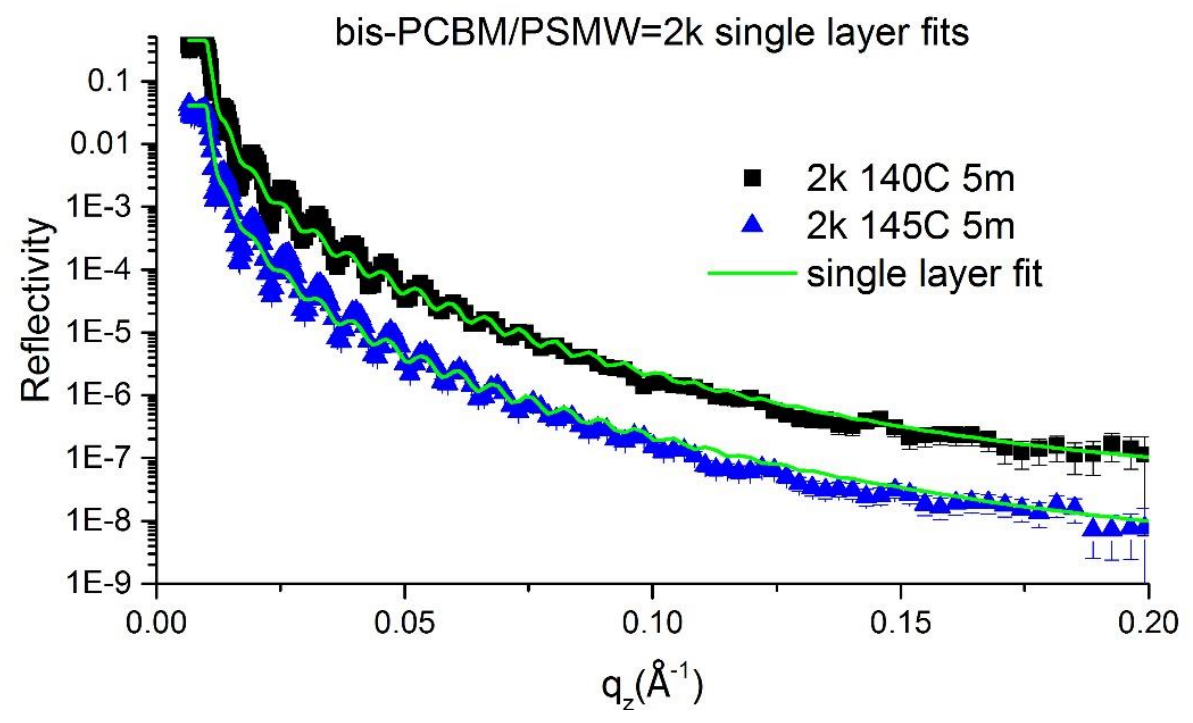

Figure 4.2-3: bis-PCBM/PS $2 k$ single layer fit: $140 C 5 \mathrm{~m}$ has a thickness of the single layer as 884 with SLD 2.196 and chisquared=40.4. 145C 5m has a thickness of the single layer as 8603 with SLD 2.15 and chi=34.1.

\subsubsection{Multilayer fits for $2 \mathrm{k}, 3.5 \mathrm{k}, 5 \mathrm{k}$ and 20k PS samples}

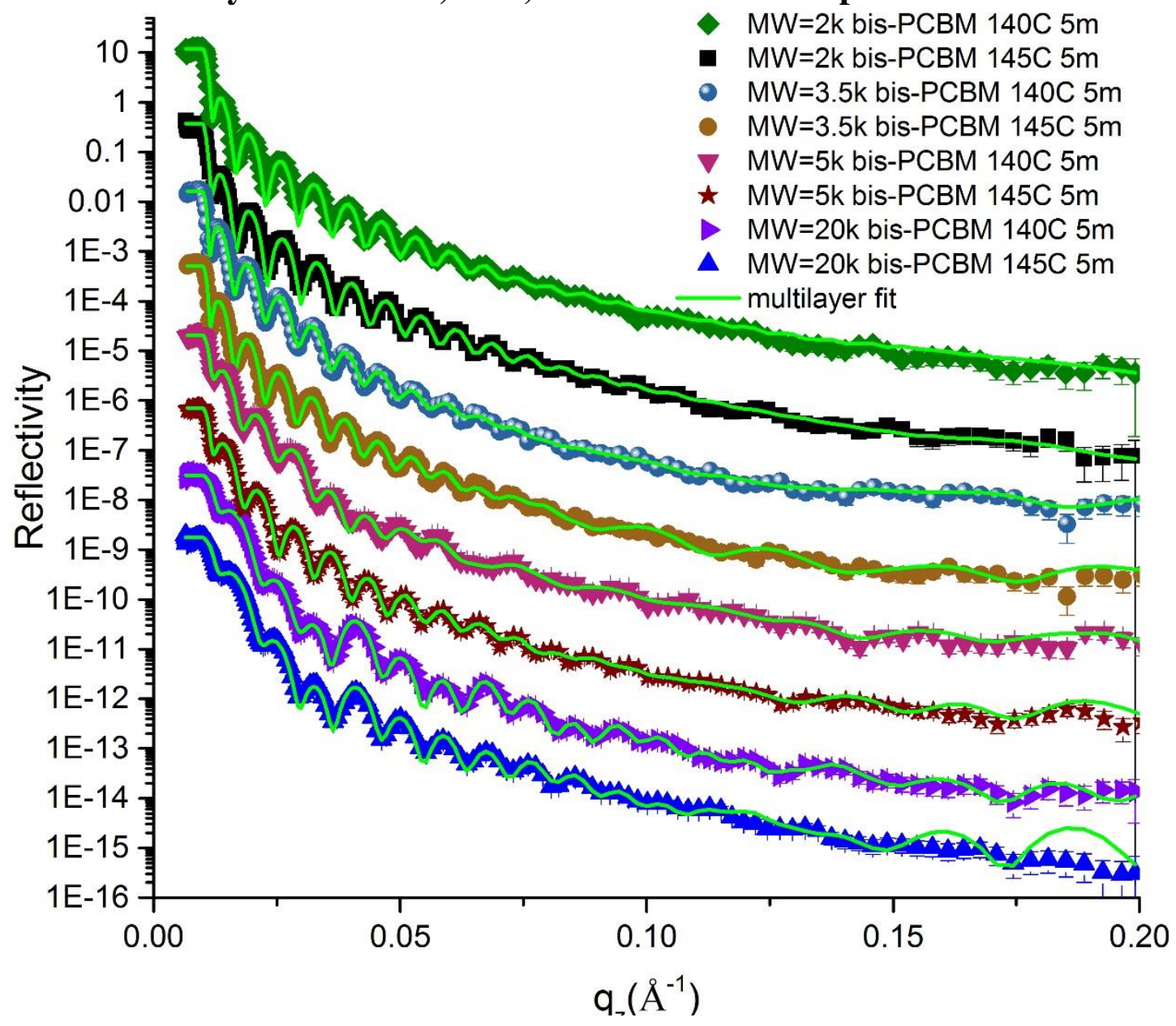

Figure 4.2-4: Multilayer fits for the bis-PSCM/PS samples indicated in the legend. 

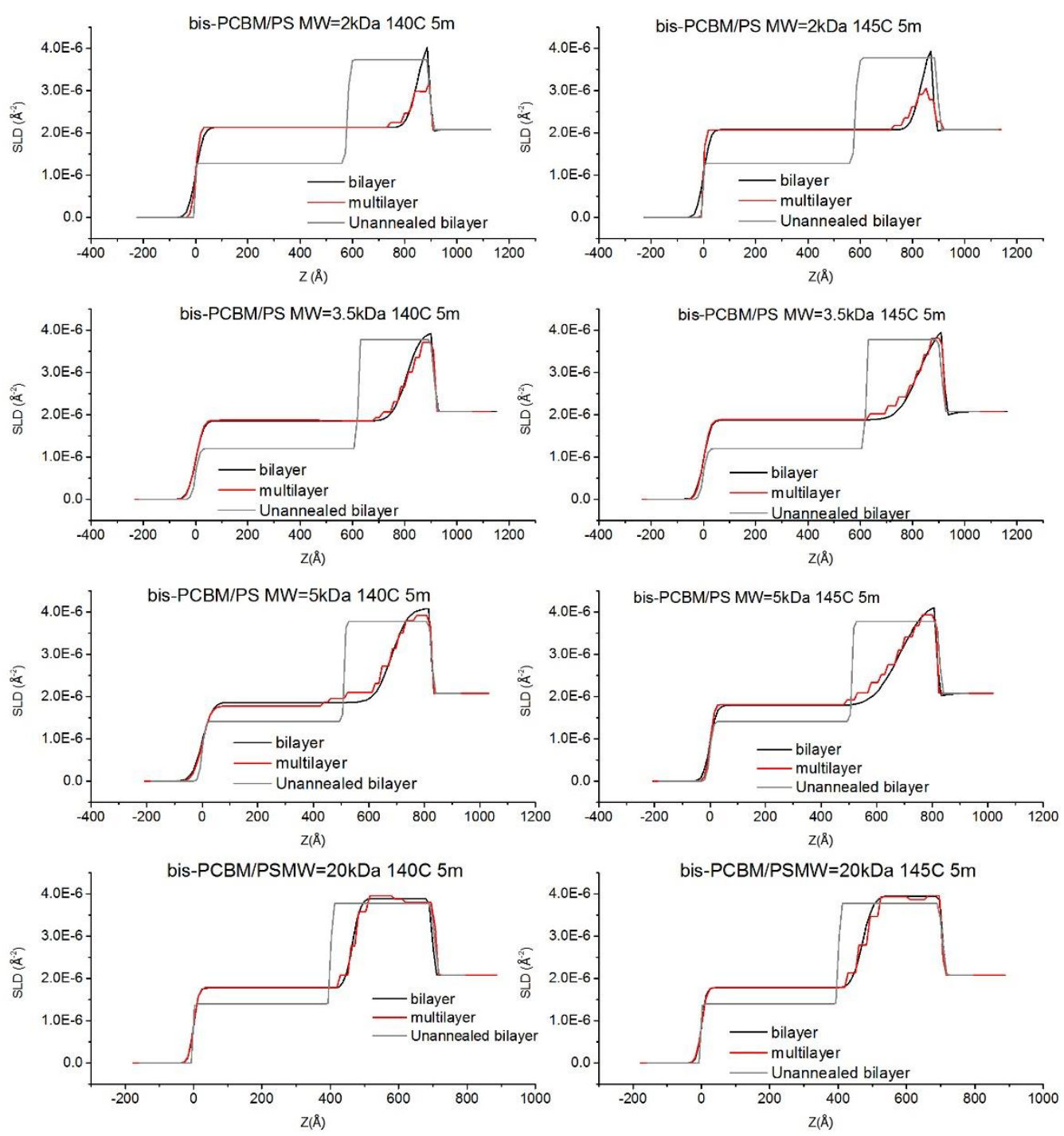

Figure 4.2-5:bis-PCBM/PS samples; multilayer and bilayer SLD profiles. The unannealed profiles are estimated from NR measurements on other (unannealed) samples.

Figure 4.2-4 and Figure 4.2-5 show the multilayer fits and SLD profiles for the $2 \mathrm{k}$, $3.5 \mathrm{k}, 5 \mathrm{k}$ and 20k bis-PCBM/PS samples. These fits used between 6 and 12 discrete layers to model the sample SLD profile. The interface roughness between each of these layers was set to zero, but the sample surface roughness was allowed to vary in the fit. Figure 4.2-5 also shows estimates of the SLD profiles for each of the samples before annealing. These estimated profiles were constructed using SLD information 
from the two unannealed bis-PCBM/PS bilayers that were measured using NR (the $2 \mathrm{k}$ sample shown in figure 4.2-1 and a 100k in-situ annealed sample shown below), and layer thickness information from a combination of AFM and NR measurements on these two samples and previous unannealed samples. The pure bis-PCBM and PS SLDs, averaged from the two unannealed bilayers, are $3.81+/-0.023 \times 10^{-6} \AA^{-2}$ and $1.31+/-0.036 \times 10^{-6} \AA^{-2}$ respectively. The multilayer fits show good agreement with the bilayer fits, clearly demonstrating a significant increase in the top layer SLD and a reduction in the thickness of the bottom layer (in comparison to the unannealed samples) for all eight samples. The SLD profiles from the multilayer and bilayer fits show very good quantitative agreement for most aspects of all samples. The $3.5 \mathrm{k}, 5 \mathrm{k}$ and 20k samples show SLD profiles which are close to error function profiles between an approximately pure bis-PCBM bottom layer and a PS-rich top layer. There are some differences in the gradients of the SLD profiles near to the buried interface between these two layers in the $3.5 \mathrm{k}$ and $5 \mathrm{k}$ samples, but the most significant difference is that the multilayer fits to both $2 \mathrm{k}$ samples are able to capture the existence of a lower maximum SLD in the bottom layer than the bilayer fits (with a significantly lower value of chi-squared; 4.67 and 3.58 compared to 6.4 and 5.57 for the bilayer fits), indicating that extensive mixing is occurring in both layers in the $2 \mathrm{k}$ samples.

The multilayer fits for the annealed samples in Figure 4.2-5 are plotted on top of one another in Figure 4.2-6. It is clear from this graph that there is a systematic progression in the mixing behaviour right across this range of $\mathrm{MW}$, with more extensive mixing in the lower MW samples. This is seen by the gradual reduction in the amount of higher SLD material in the bottom layer with decreased MW, and a corresponding increase in the top layer SLD at lower MW. The implications of these ex-situ measurements, will be discussed following the next section on in-situ measurements, and a brief section on measurements at a significantly higher temperature $\left(180^{\circ} \mathrm{C}\right)$. 


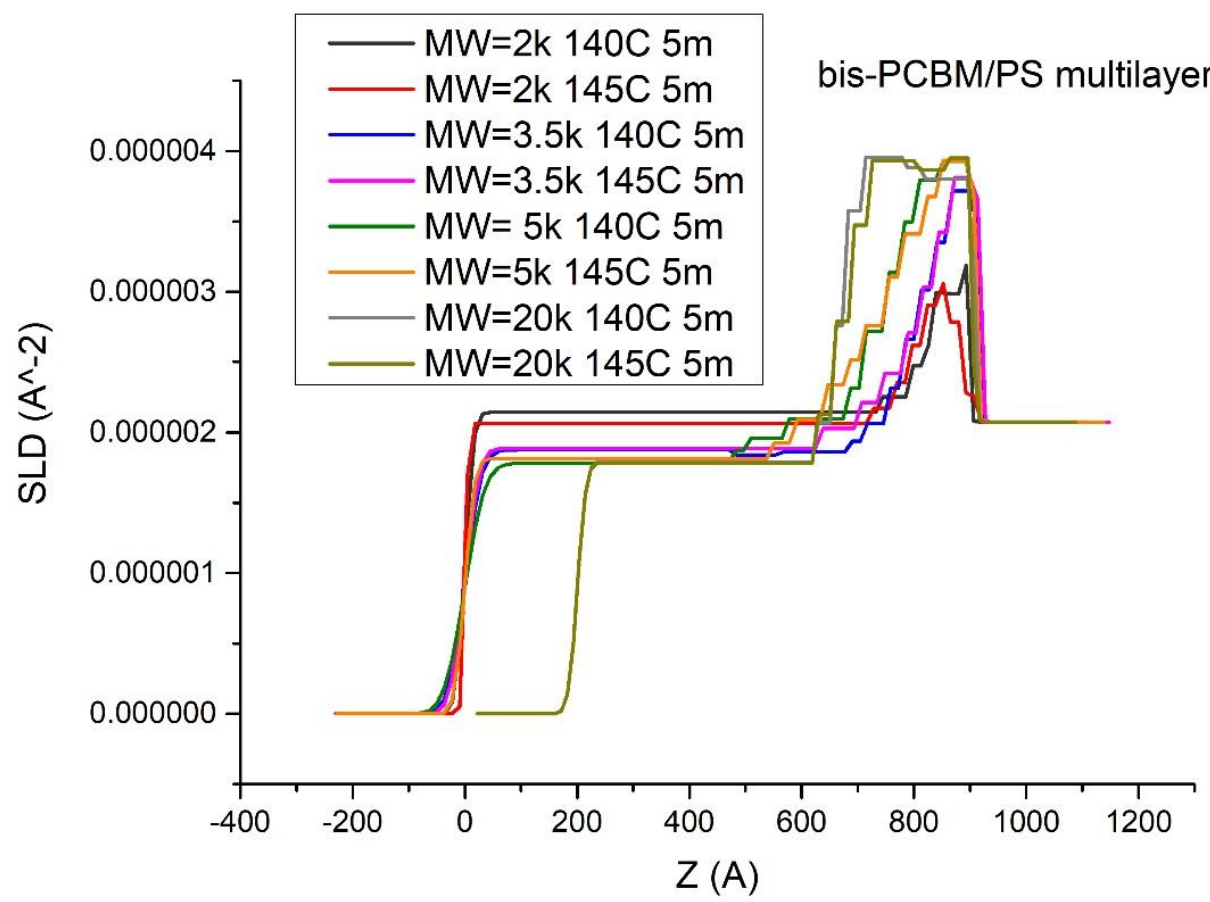

Figure 4.2-6: Multilayer fits of the bis-PCBM/PS bilayers for the molecular weights detailed in the legend.

\subsubsection{In-situ 100k PS sample}

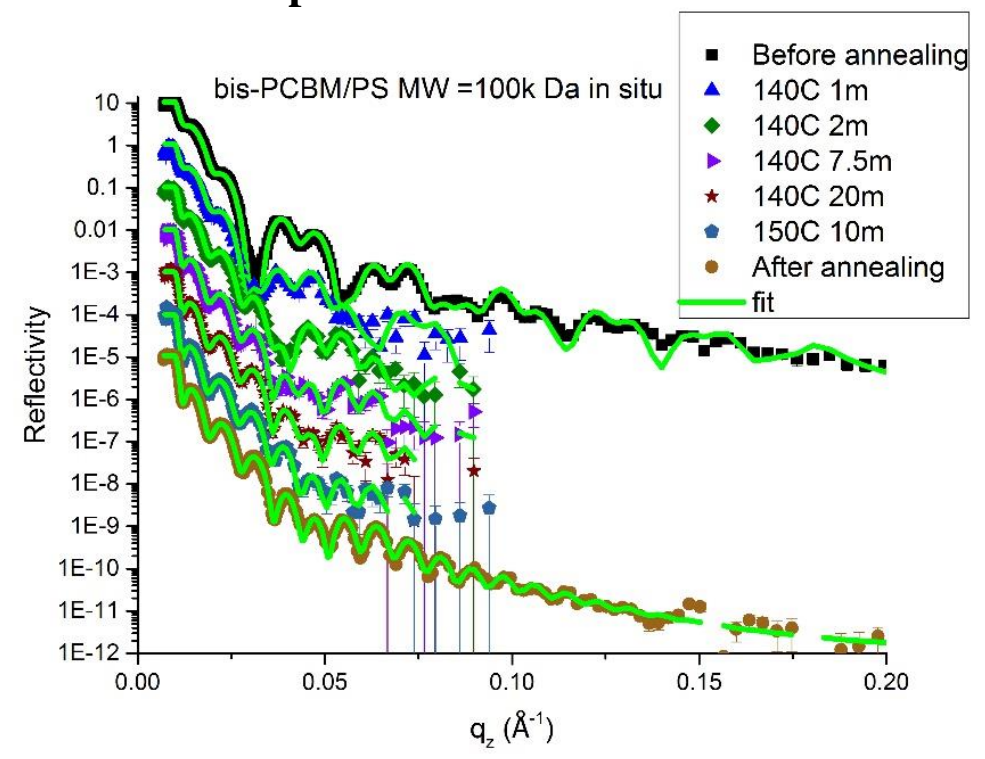

Figure 4.2-7: This shows the reflectivity curves for a bis-PCBM/PS 100k bilayer showing the full reflectivity curves before and after annealing as well as 30s 'kinetic' measurements taken during annealing showing the gradual change in the reflectivity curve. 


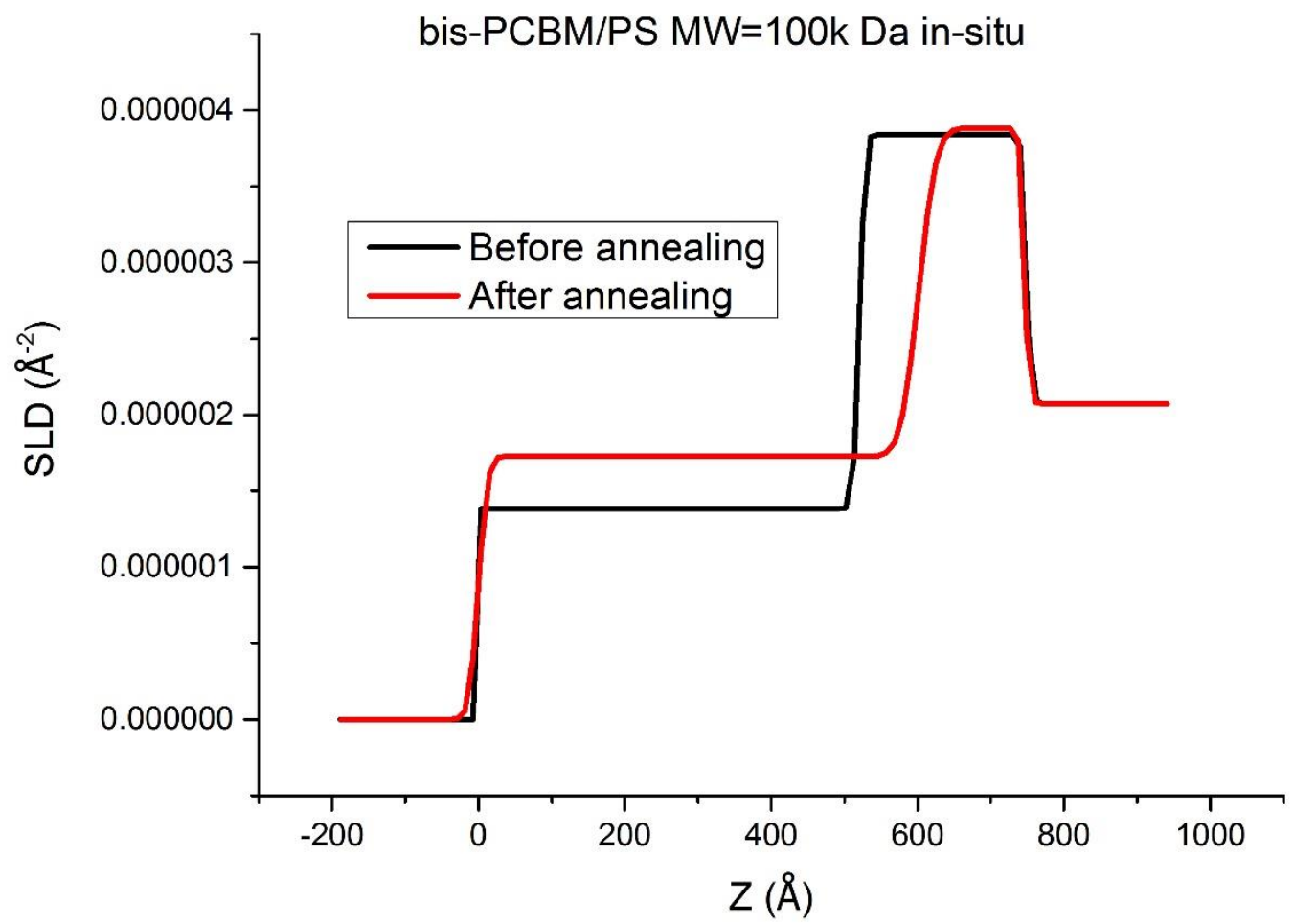

Figure 4.2-8: SLD profiles corresponding to the fits before and after in-situ annealing from figure 4.2-7.

Figure 4.2-7 and Figure 4.2-8 show the data from the in-situ experiment on a bisPCBM/PS 100k sample. Very good fits are obtained for both the full reflectivity curves and the kinetic measurements, and optical micrographs show very few lateral inhomogeneities on this sample. Figure 4.2-7 shows that there is a gradual change in the shape of the reflectivity curve, as the system moves towards equilibrium. As with the ex-situ annealed samples, the SLD profiles show that the interface broadens and there is significant diffusion of the bis-PCBM into the PS top layer, indicated by the depletion of the bottom layer and the raised SLD of the top layer after annealing. The SLD profiles before and after annealing in Figure 4.2-8 also show very good conservation of material (integrating the scattering length density over both SLD profiles, gives a ratio of the scattering length per unit area of the sample before:after annealing of $0.99: 1)$. 


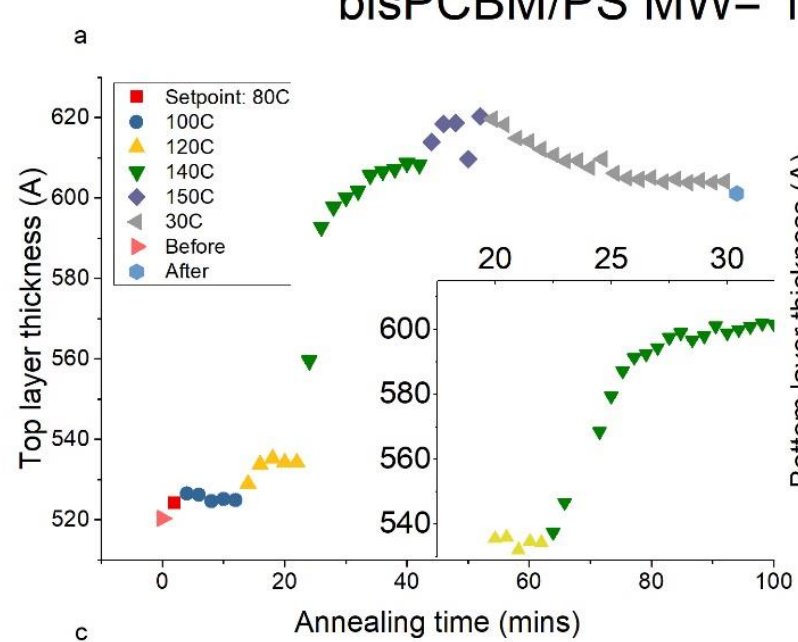

bisPCBM/PS MW= $\underset{b}{100 k}$ Da in-situ fit parameters
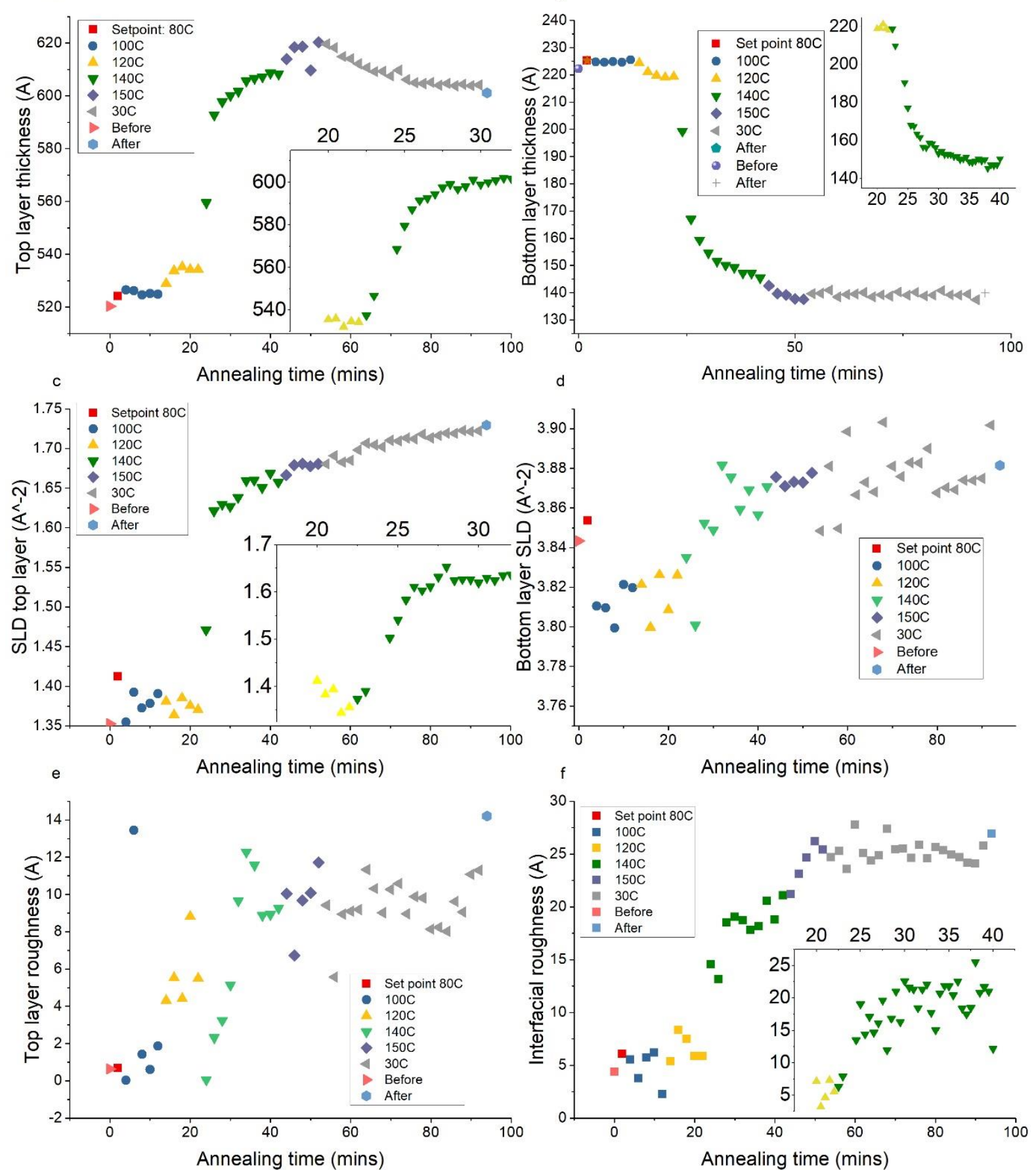

Figure 4.2-9: The change in fit parameters over time for bisPCBM/PS MW=100kDa. Each data point is for reflectivity summed over four 30s measurements barring the inset graphs which show data points for individual 30s measurements. Graph a is thickness of the top layer (PS-rich), graph $b$ is the thickness of the bottom layer (PCBM), graph $c$ is the SLD of the top layer, $d$ is the SLD of the bottom layer, $e$ is the top layer roughness and $f$ is the interfacial roughness.

Figure 4.2-9 shows the change in the fit parameters for the kinetic measurements. The top layer thickness increases as the bottom layer decreases in thickness between 
the initial and final phase. The top layer increases in thickness by $80 \AA$ and the bottom layer decreases by $75 \AA$. The top layer SLD also increases significantly from 1.35 to 1.7 . This indicates $25 \%$ bis-PCBM (details of this calculation are given in section 4.2 .5 below) in the top layer after annealing. There is some scatter in the measurement of the bottom layer SLD and the two roughness parameters in the kinetic measurements, but the plots enable an assessment of the timescales and temperature dependence of the fullerene diffusion and interface broadening processes. The interfacial roughness increases from 5 to $25 \AA$. The most significant changes in the parameter values occur after the sample is heated to $140^{\circ} \mathrm{C}$ and then remain comparatively stable through further temperature increase to $150^{\circ} \mathrm{C}$ and cooling (apart from changes to the top layer SLD and thickness that are commensurate with thermal contraction on cooling). This finding, the fact that the sample surface temperatures for the ex-situ annealed samples are intermediate between the sample surface temperatures reached in the in-situ measurements at setpoints $140^{\circ} \mathrm{C}$ and $150^{\circ} \mathrm{C}$ (ex-situ surface temperatures are $137{ }^{\circ} \mathrm{C}$ and $142{ }^{\circ} \mathrm{C}$, in comparison to in-situ temperatures of $134{ }^{\circ} \mathrm{C}$ and $144{ }^{\circ} \mathrm{C}$, all with a margin of error of $+/-2.2{ }^{\circ} \mathrm{C}$; see Table 1 and Table 2 in section 3.12), and the very similar SLD profiles shown in Figure 4.2-6 for ex-situ samples at set-points of $140{ }^{\circ} \mathrm{C}$ and $145^{\circ} \mathrm{C}$, means that it is reasonable to propose that both the ex-situ samples and the in-situ sample (after annealing) all have composition profiles that are at thermodynamic equilibrium within that range of temperatures. 


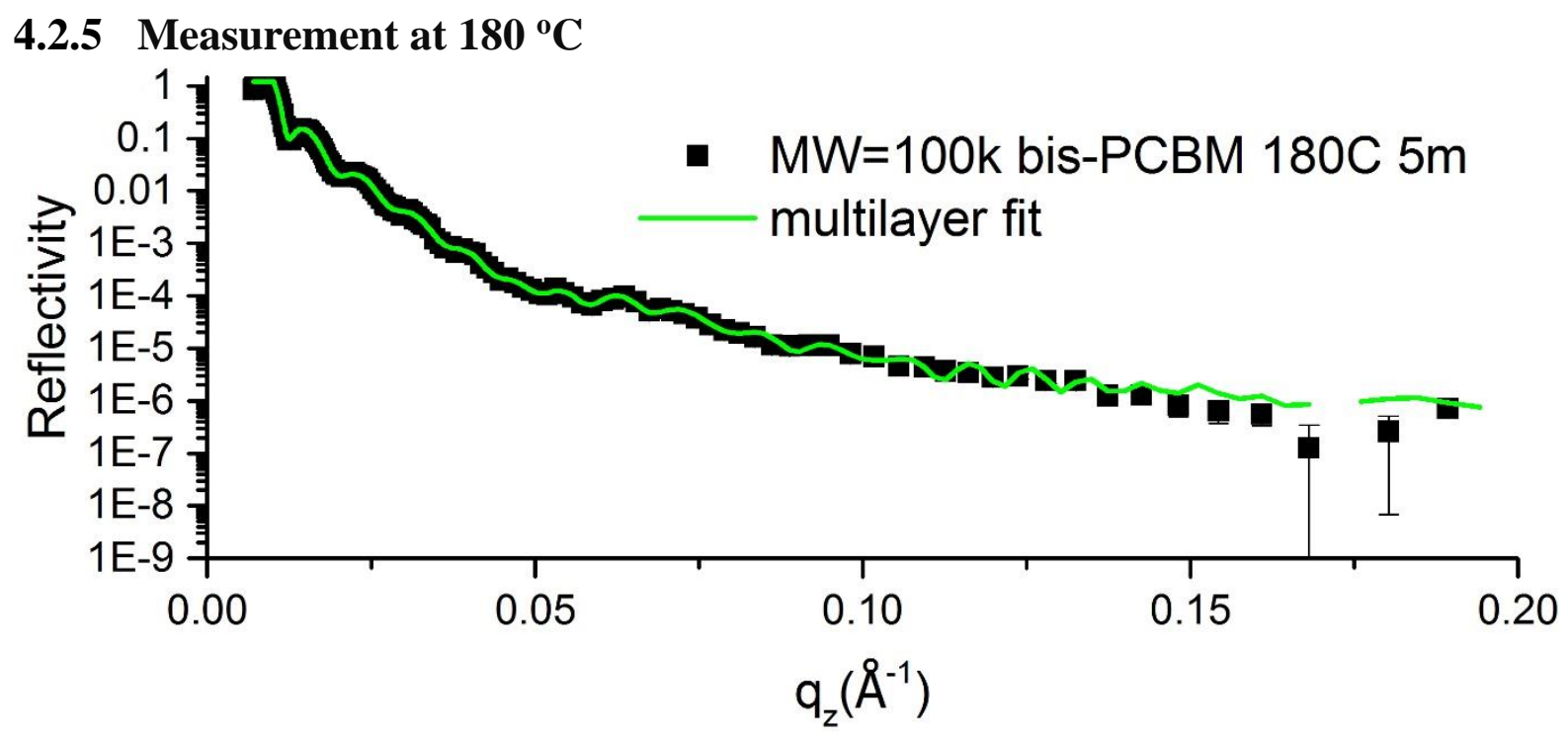

Figure 4.2-10: Multilayer fit to data.

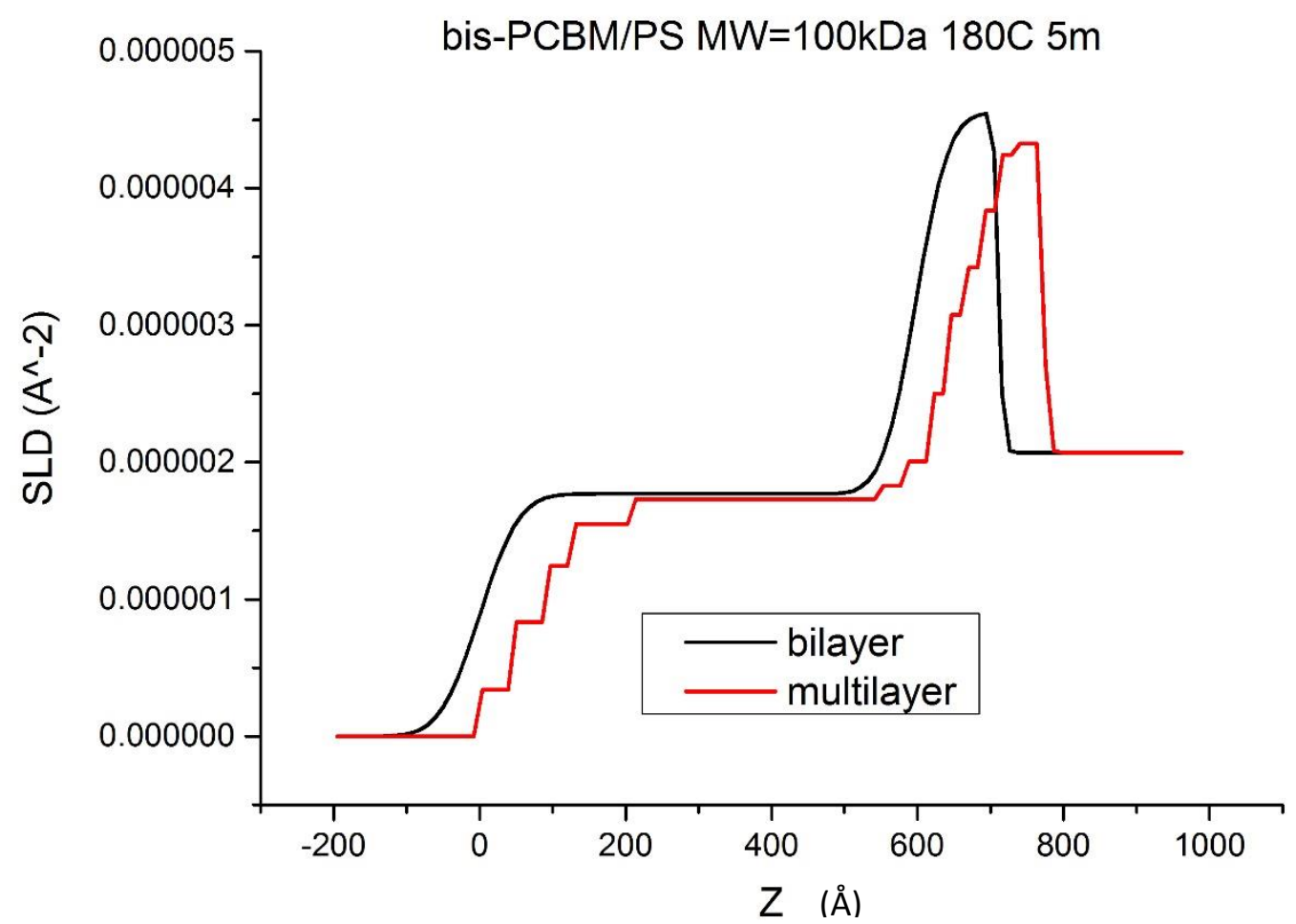

Figure 4.2-11: Comparison of bilayer fit with surface and interfacial roughness to multilayer fit with no roughness.

Figure 4.2-10 and Figure 4.2-11 show the multilayer fit for the 100k sample annealed at $180{ }^{\circ} \mathrm{C}$, and the SLD profile for this and the bilayer fit. The multilayer fit more accurately reproduces the fringes although there is little difference in the SLD profiles. Chi-squared is improved in the multilayer fit at 2.58 compared to 4.58 for 
the bilayer fit. The reflectivity curve shows that the size of the fringes is significantly reduced in comparison to the samples annealed at lower temperature. The fits show that this is due to a low gradient in SLD between the top layer and a thin bottom layer. The bilayer fit indicates that in this sample the interfacial roughness, at $50.3 \AA$, is larger than the 20k samples (annealed at $140{ }^{\circ} \mathrm{C}$ and $145^{\circ} \mathrm{C}$ ) or the in-situ $100 \mathrm{k}$ sample (these values are given in section 4.2.4). The broad interface is not due to lateral inhomogeneities on this sample, as can be seen from the excellent uniformity in this sample (see appendix at the end of this chapter for a typical optical micrograph). It is therefore proposed that this is an effect of the significantly elevated annealing temperature of this sample. There was no measurable temperature dependence observed in the PCBM/PS system (with ex-situ annealing up to $170{ }^{\circ} \mathrm{C}$ ) but temperature could in principle have an effect on the bis-PCBM/PS system (which is expected to have a higher mixing compatibility in comparison to PCBM/PS). However, we only have one measurement to-date and so this finding needs to be checked for reproducibility. Given this, the behaviour of this one sample at $180{ }^{\circ} \mathrm{C}$ will not be included in the discussion in section 4.2 .5 below.

\subsubsection{The influence of PS MW on layer composition and interfacial roughness in bisPCBM/PS bilayers.}

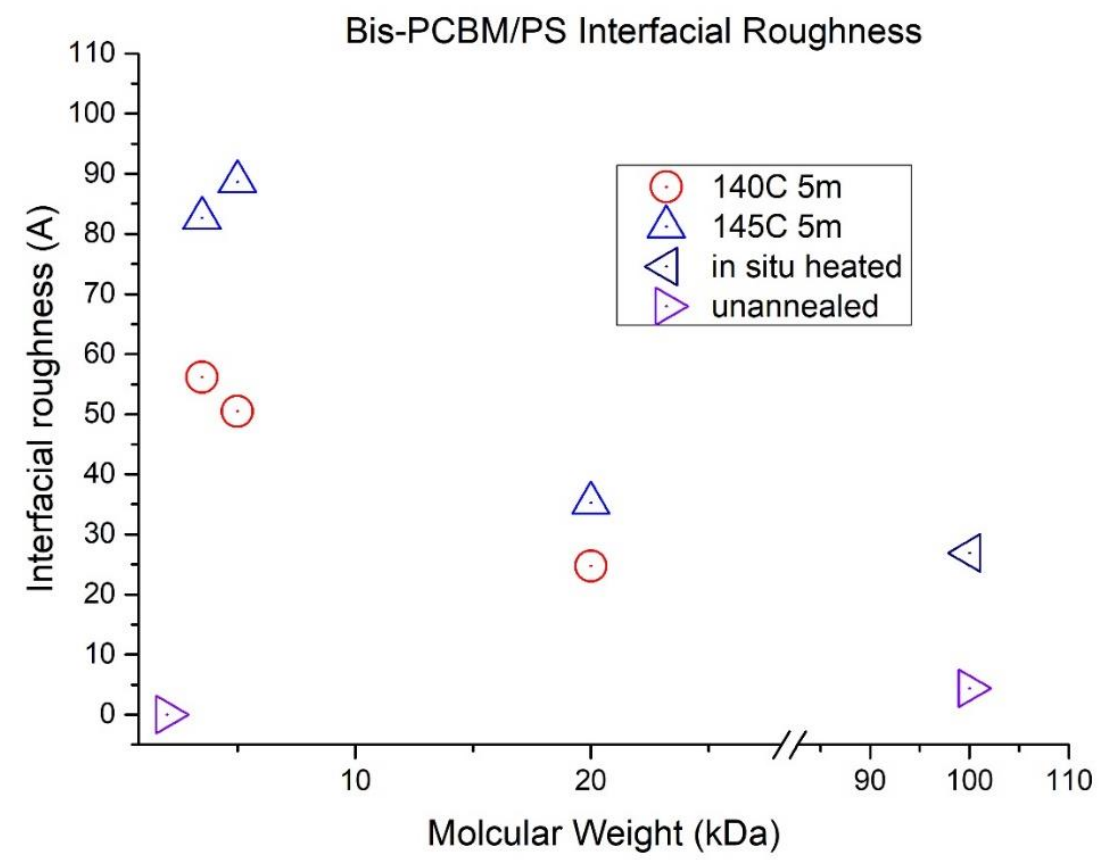

Figure 4.2-12: Interfacial roughness from bilayer fits as a function of PS molecular weight for bis-PCBM/PS bilayers. Parameters for the $2 k$ samples are not included. 
Figure 4.2-12 shows the interfacial roughness from the bilayer fits to the bis-PCBM samples over a range of MW. This shows similar qualitative behaviour to the PCBM/PS samples, although the interfacial roughness is becoming comparable to the thickness of the bis-PCBM layer itself for low MW (5k and below), indicating that there may not be enough bis-PCBM present to fully observe the tanh profile predicted to exist between two uniform layers at equilibrium. Despite this caveat, some insight may be gained regarding the behaviour of this system in comparison to F-H theory by examining the composition of the top (PS-rich) layer and the maximum SLD value in the bottom layer, as a function of MW. The 20k and 100k PS samples all have interfacial roughness that is larger than for the PCBM/PS system for these MWs.

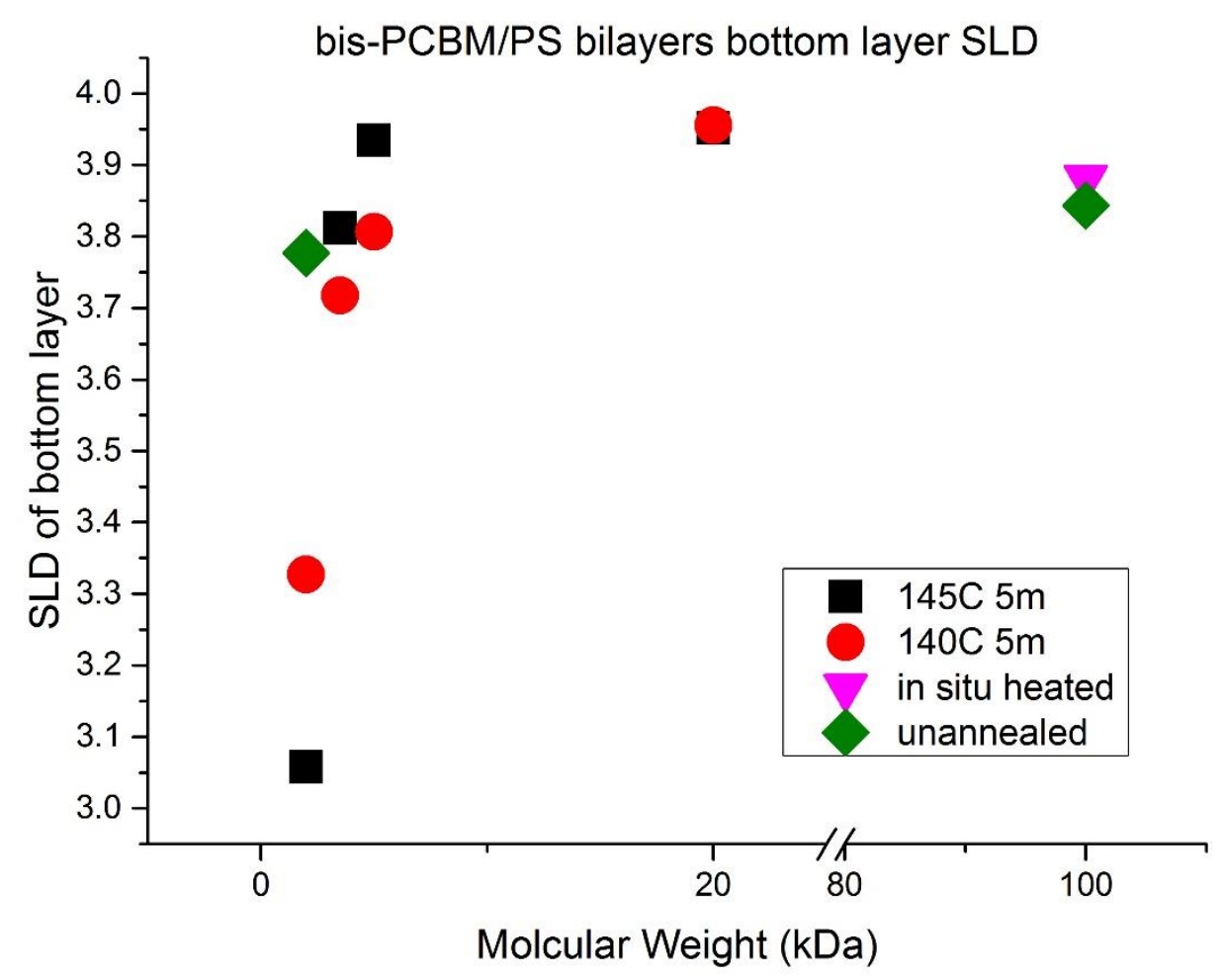

Figure 4.2-13: SLD values for the bottom layer as a function of molecular weight (taken from the maximum value of the multilayer fits).

Figure 4.2-13 shows the maximum value of the SLD profile from the the multilayer fits to the ex-situ annealed samples, and the bilayer fit to the unannealed and in-situ annealed samples. This shows that the $2 \mathrm{k}$ samples have significantly more mixing in the bottom layer in comparison to the other samples. This indicates an MW 
dependence which will be examined theoretically below by using Flory-Huggins theory. The bottom layer SLD is constant for MW above $2 \mathrm{k}$ as high as $100 \mathrm{k}$. For the $2 \mathrm{k}$ samples, the maximum SLD value from the multilayer SLD profile gives a maximum composition of 74+/-3.69\% bis-PCBM present in the bottom layer using equation (4.2). For the $3.5 \mathrm{k}$ samples there is $98+/-2.7 \%$; for the $5 \mathrm{k}$ : $100+/-2.7 \%$; for $20 \mathrm{k}$ and $100 \mathrm{k}$ combined the mean is $100+/-2.5 \%$ for bis-PCBM present in the bottom layer.

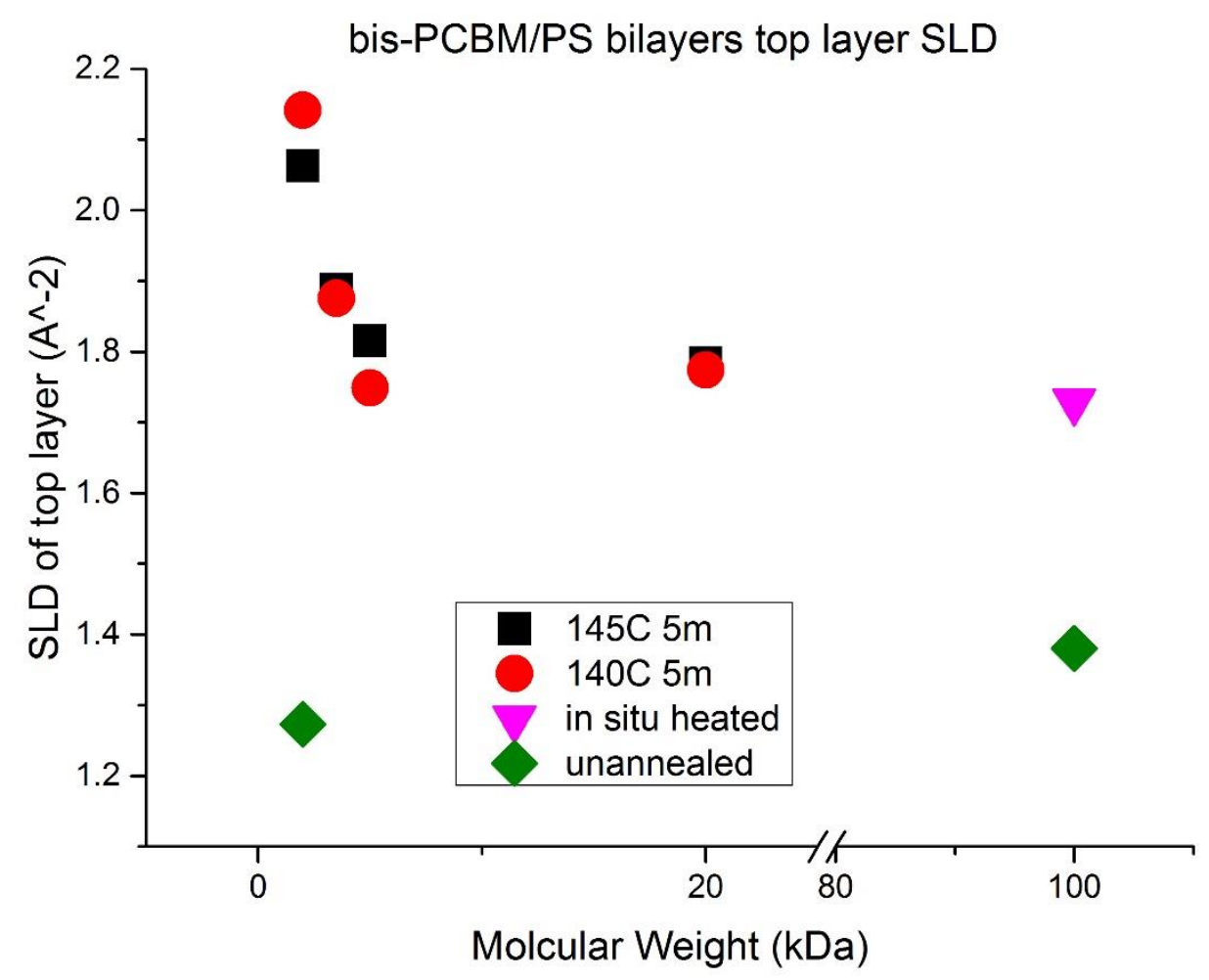

Figure 4.2-14: SLD of the peak of the top layer in initially bis-PCBM/PS bilayers taken from the multilayer fits. The legend indicates the annealing temperature and time.

Figure 4.2-14 shows the top layer SLD of the bis-PCBM/PS bilayers taken from multilayer fits. As with the bottom layer SLD there is a significantly different value for the $2 \mathrm{k}$ samples. In this case, these samples have a higher SLD indicating diffusion of the bis-PCBM into the top layer. These values indicate a mean value of $18.3+/-1.6 \%$ bis-PCBM in the top layer for the 20k to 100k PS samples (combined), $18.8+/-1.7 \%$ for $5 \mathrm{k}, 22.8+/-1.5 \%$ for $3.5 \mathrm{k}$, and $31.7+/-2 \%$ in the $2 \mathrm{k}$ samples. 
The percentage of bis-PCBM in a particular layer was calculated from the SLD of that layer, and the pure material SLDs as follows;

$$
\%_{\text {bis-PCBM }}=\frac{S L D_{\text {layer }}-S L D_{\text {pure } P S}}{S L D_{\text {pure bis }-P C B M}-S L D_{\text {purePS }}} \times 100 .
$$

The SLD value for pure bis-PCBM was taken as the average measured from unannealed bilayers as $3.81+/-0.032 \times 10^{-6} \AA^{-2}$. The SLD value for the pure PS layer was taken as the average value from several measurements as $1.31+/-0.071 \times$ $10^{-6} \AA^{-2}$.

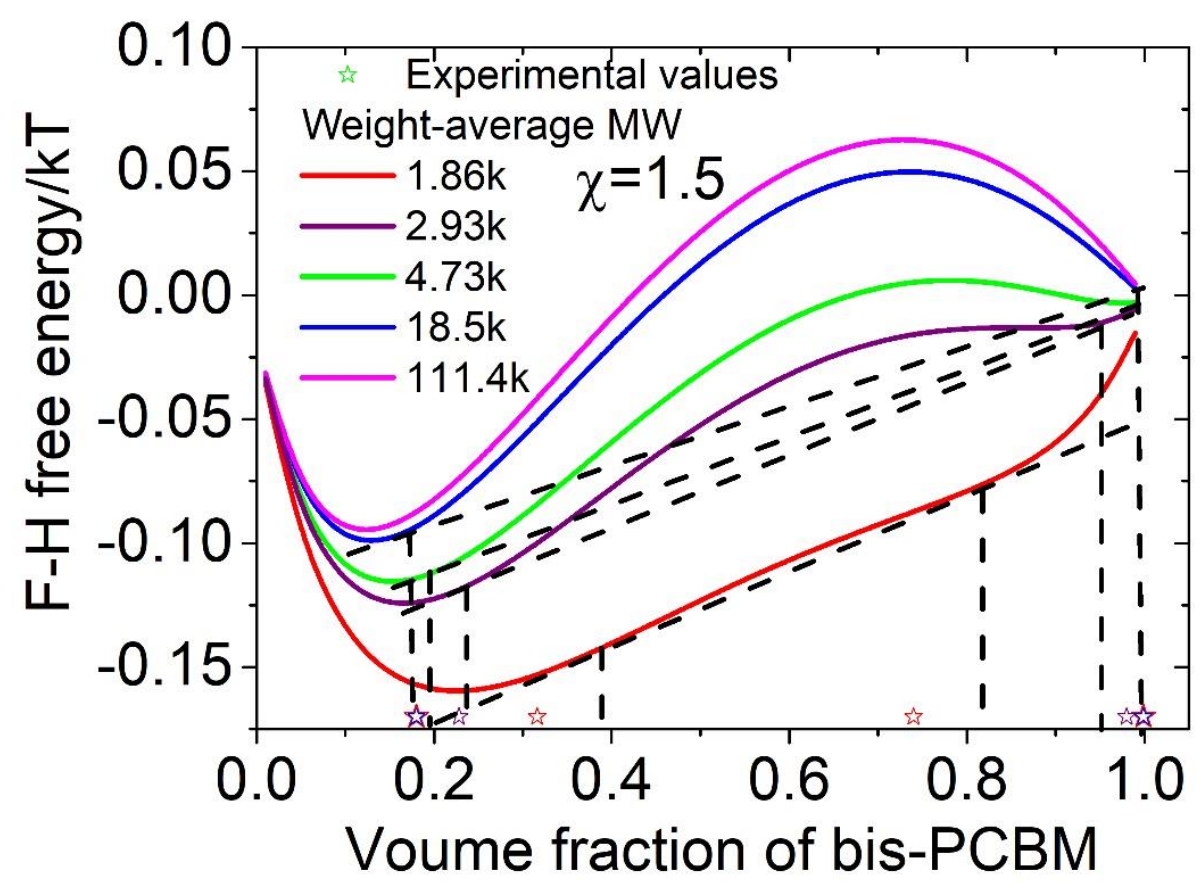

Figure 4.2-15: The Flory-Huggins free energy of mixing of a PS/bis-PCBM mixture with the lattice size set equal to the size of a bis-PCBM molecule for chi=1.5. The stars indicate experimentally measured compositions.

This behaviour could be accounted for by Figure 4.2-15 which shows a F-H free energy curve for chi=1.5 with the experimentally measured compositions also shown. The lattice size for this calculation was taken to be the volume of a bisPCBM molecule, $1.3 \mathrm{~nm}^{3}$ (meaning that the chi parameter here is referenced to a $30 \%$ larger volume, but with a reduced number of PS 'monomers' per chain than for the PCBM/PS calculations). This was calculated using a density of $1.41 \mathrm{~g} / \mathrm{cm}^{3}$ (determined using the NIST SLD calculator (Kienzle 2016) with the mean pure bisPCBM SLD of $3.81 \times 10^{-6} \AA^{-2}$ ) and the bis-PCBM MW of 1101.1Da. The free 
energy curves were found by first choosing chi to account for the observed compositions at higher MW (20k and 100k). The free energy curves for $2 \mathrm{k}, 3.5 \mathrm{k}$ and 5k PS shown in Figure 4.2-15 are predictions, using this same value of chi.

The common tangent constructions are drawn in, indicating the level of mixing that would be expected for this chi-value for a range of MW. The vertical dashed lines in Figure 4.2-15 show the theoretical co-existing compositions. Comparison with the experimentally determined layer compositions (the star symbols in Figure 4.2-15) shows that Flory-Huggins theory with chi set to 1.5 captures the magnitude of the changes with MW reasonably well. Overall it has been found that bis-PCBM/PS systems behave in a qualitatively similarly way to PCBM/PS systems with the formation of two coexisting compositions and increased interfacial roughness for lower MW. This system clearly has a more pronounced MW dependence than PCBM/PS, making it easier to observe the MW dependent behaviour given the sensitivity of NR. To compare chi values between the two systems we can rescale the bis-PCBM chi value of 1.5 by recalculating for the same lattice size as was used for the PCBM/PS calculation. This gives a chi value for bis-PCBM/PS referenced to the size of a PCBM molecule of 1.15. This is considerably lower than the value found for $\mathrm{PCBM} / \mathrm{PS}$, which was 2 , as is expected given the extra side chain which improves compatibility with PS. This lower value of chi is qualitatively consistent with a higher interface roughness for the bis-PCBM/PS system, which is predicted theoretically, and measured experimentally.

\subsubsection{Overall conclusions for bis-PCBM/PS and PCBM/PS}

The results of the experiments presented in Sections 4.1 and 4.2 of the measured interfacial roughness and layer composition are now discussed in relation to F-H theory for polymer/small-molecule mixtures and SCFT for polymer/polymer interfaces. The key findings are summarised as follows:

- Both the bis-PCBM/PS and the PCBM/PS systems show behaviour that is indicative of a liquid-liquid equilibrium between two coexisting phases. This is by displaying, qualitatively, what you would expect as a function of MW. The different systems show different aspects of this behaviour: the PCBM/PS system shows very little change in composition as a function of MW while the interfacial roughness increases for lower MW; the bis-PCBM/PS system shows, again, increased interfacial roughness as MW is decreased and also 
more dramatic mixing behaviour with mixing occurring to a larger extent in both layers at low MW. There is also the observation of a steady state of coexisting compositions after compositional evolution occurring in under two minutes (observed in both in-situ and ex-situ measurements). This indicates equilibrium in the absence of extensive crystallisation.

- F-H theory shows reasonable agreement with both systems for predicting the magnitude of the change in layer compositions across a range of MW.

- By comparing chi values for these systems (referenced to the same lattice size and extracted by accounting for compositions observed at high MW) we see a significantly lower value for the bis-PCBM/PS system. This is as expected given that the bis-PCBM has an extra side-chain making it more chemically similar to PS.

- The interfacial roughness of the bis-PCBM/PS system also shows behaviour that is expected for a lower chi value: broader interfaces. This agrees with SCFT theory qualitatively when comparing high MWs (20k and 100k) in both systems where nice bilayer fits are obtained.

- The prediction of Broseta et. al (Daniel Broseta et al. 1990) (see Figure 2.6-2) gives the prediction for $\chi \mathrm{N}$ for asymmetric $\mathrm{N}$ across the interface. The Tang and Freed prediction for symmetric $\mathrm{N}$ is shown in Figure 2.6-3. However, neither prediction applies in the limit of either $\mathrm{N}$ going to 1 . This is because of the importance of fluctuations in small-molecule systems in comparison to polymer systems (high N)(R. A. L. Jones and Randall W., Richards, 1999). This importance is captured quantitatively by the Ginzburg criterion (see equation (2.50)), which is satisfied more readily by small molecules than polymers. Fluctuations are of more importance in finite systems, and can therefore be expected to have particular relevance near interfaces. Joanny (Joanny 1978) looks at asymmetric $\mathrm{N}$ across the interface in the limit that one $\mathrm{N}$ goes to 1 . He found that in this limit the Ginzburg criterion is satisfied, meaning that mean-field theory fails and fluctuations must be taken into account. Figure 2.6-3 has no adjustable parameters and is plotted using an 'effective' statistical segment length of PS, the chi value extracted from F-H theory and $\mathrm{N}$ set to two different possible values; the first possible value has $\mathrm{N}$ set equal to the number of monomers in a PS chain (referenced to the 
PCBM molecular size) and the second has $\mathrm{N}$ set equal to $N_{\text {ave }}$ (defined as $4\left(N_{A}{ }^{-1 / 2}+N_{B}{ }^{-1 / 2}\right)^{-2}$, for a binary mixture of chains with $N_{A}$ and $N_{B}$ monomers, and also referenced to the PCBM molecular size). The magnitude of the roughness predicted in Figure 2.6-3 is a factor of around 3 lower than that found experimentally. This is the case even for the highest MWs and is in contrast to the findings of Sferrazza et. al (Sferrazza et al. 1997) who found good agreement between the theory proposed by Helfand and Tagami/Tang and Freed (in the limit of high MW) taking into account thermal capillary waves. However the percentage changes in interfacial width with MW predicted by Tang and Freed are in reasonable agreement with the observations in Figure 4.1-30 and Figure 4.2-12.

- The fact that changes in composition and interfacial width for different MW in these fullerene/PS systems can almost be described quantitatively using mean-field theory is remarkable. This indicates that, while composition fluctuations are predicted to be important here, mean-field theories (in which fluctuations are ignored) can still describe the experimental findings reasonably well. It may be that the large size of the fullerene molecules plays a role in reducing the effects of fluctuations. An important observation is that there is very little off-specular scattering in these systems, suggesting that scattering from long wavelength capillary waves (on the order of microns) is not important here. Sferrazza et. al discuss the suppression of long wavelength (and high amplitude due to equipartition of energy) capillary waves in thin films. Could similar arguments apply to the polymer/fullerene systems of this thesis, and composition fluctuations be suppressed by dispersion forces? It may be that fluctuations are affecting the interfacial width but are simply not showing up in the off-specular scattering. Typical correlation lengths of composition fluctuations in polymer blends and solutions are on the order of a few nm to tens of nm (R. Colby; M. Rubinstein; 2003) with a recently reported value for PCBM/PS (in a 10\% PCBM miscible blend) of $60+/-40 \mathrm{~nm}$ (Bernardo et al. 2016). However, the in-plane distances to which the offspecular is sensitive, are a few hundred nm to microns. This means that composition fluctuations of the scale of the correlation length or less in the plane of the sample, will not show up in off-specular scattering, but fluctuations 
normal to the substrate may affect the specular reflectivity and contribute to the measured interfacial roughness.

To conclude, our results for both PCBM/PS and bis-PCBM/PS systems indicate that we have achieved liquid-liquid equilibrium in these systems and that our use of F-H theory is valid. Both systems exhibit different aspects of expected behaviour with MW for a liquid-liquid equilibrium, i.e. increased interface broadening and increased miscibility with lower MW. There is qualitative agreement with polymer/polymer interfacial width predictions with lower MW giving broader interfacial widths. This has been seen in both the PCBM/PS and bis-PCBM/PS systems. These observations might be useful in future developments of theory in describing these systems.

The above findings can be set in context with-respect-to the OPV community by looking at other recent efforts to gain fundamental understanding of OPV materials from the point of view of equilibrium thermodynamics. Ye et al (Ye et al. 2018) recently studied mixing in model bilayer systems involving conjugated polymers and small molecules (focusing on PCDTBT:PCBM). They uncovered a strong correlation between device performance and fundamental parameters characterising the miscibility in polymer/small-molecule systems. In their study they used FloryHuggins theory to interpret domain compositions and quantitatively link the FloryHuggins interaction parameter to performance as a function of the polymer MW, and also for a broader range of polymer/small-molecule devices. Ye et al are seeking to enable rational OPV design by linking fundamental behaviour such as is described by F-H theory, to device performance. The work presented in this thesis also adds to such understanding, but the utilisation of well-understood and well-controlled model polymers allows a more detailed investigation of MW-dependent behaviour (because of the much lower polydispersity in PS, compared to typical conjugated polymers), enabling the in-depth study of interfacial width, as well as layer composition (the focus of the study by Ye et al). Taking these results of correlation between mixing and OPV device performance in conjunction with this thesis' findings of behaviour for a model system will potentially enable further developments of theory in relation to polymer/small molecules and therefore tuning of device performance by parameters such as MW. 


\subsubsection{Appendix}

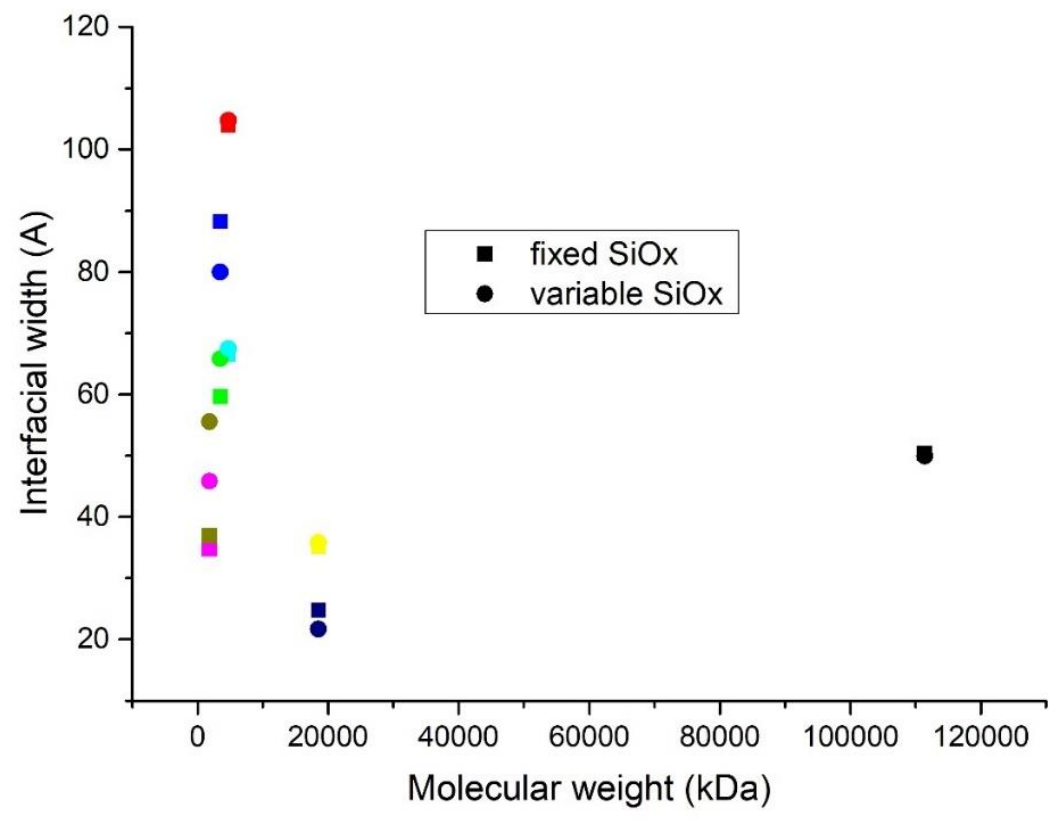

Figure 4.2-16: Parameter values for fixed and varied oxide layers. The same colour represents the same sample.

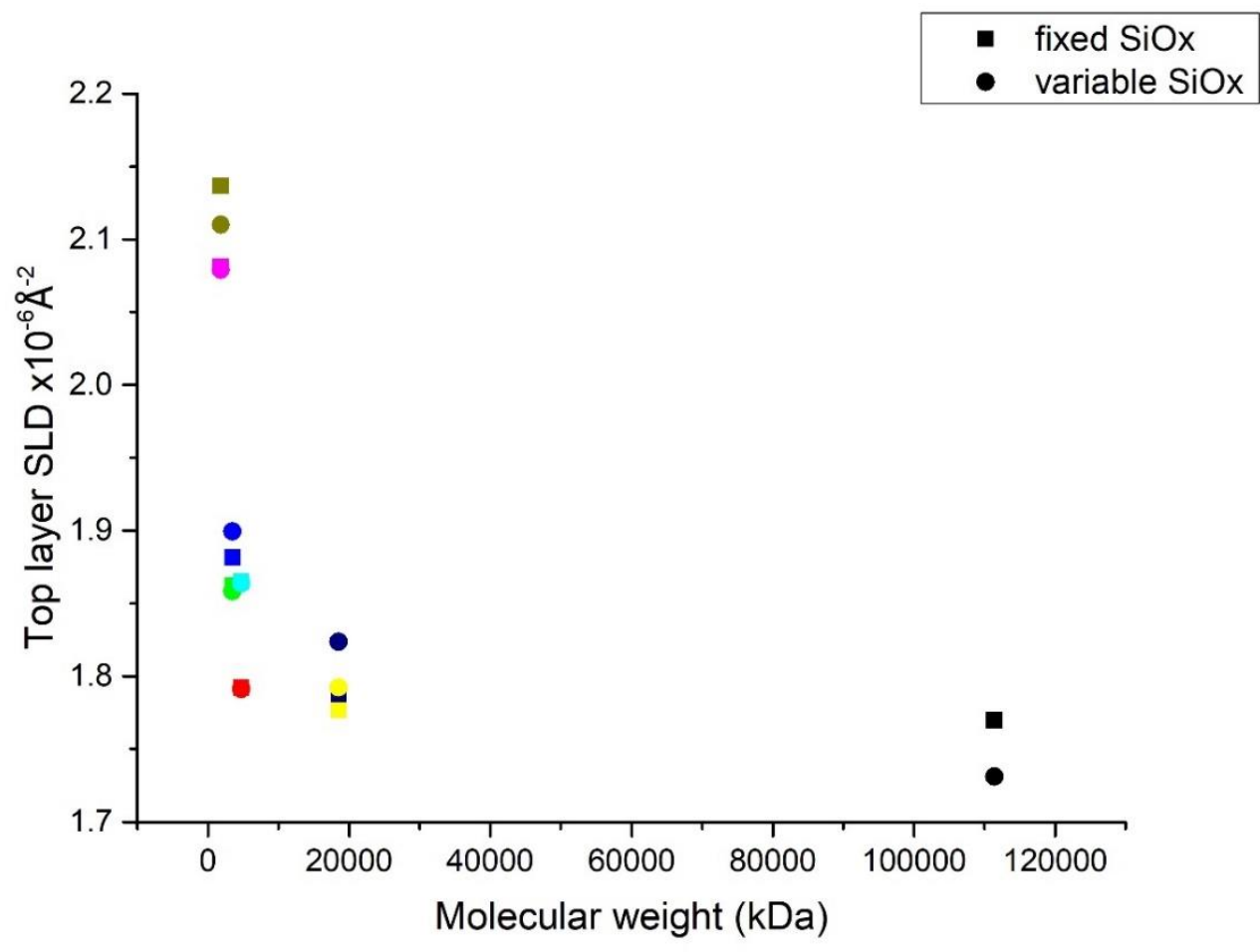

Figure 4.2-17: Parameter values for fixed and varied oxide layers. The same colour represents the same sample. 


\section{Sample List}

Table 11 shows the NR sample list showing which samples were measured at which reflectometers.

\begin{tabular}{|l|l|l|}
\hline INTER & Figaro & D 17 \\
\hline $2 \mathrm{k} 145^{\circ} \mathrm{C} 5 \mathrm{~m}$ & & $100 \mathrm{k} 180^{\circ} \mathrm{C} 5 \mathrm{~m}$ \\
$20 \mathrm{k} 145^{\circ} \mathrm{C} 5 \mathrm{~m}$ & & $100 \mathrm{k}$ insitu \\
$2 \mathrm{k} 140^{\circ} \mathrm{C} 5 \mathrm{~m}$ & & \\
$20 \mathrm{k} 140^{\circ} \mathrm{C} 5 \mathrm{~m}$ & & \\
$2 \mathrm{k}$ unannealed & & \\
$5 \mathrm{k} 145^{\circ} \mathrm{C} 5 \mathrm{~m}$ & & \\
$3.5 \mathrm{k} 140^{\circ} \mathrm{C} 5 \mathrm{~m}$ & & \\
$3.5 \mathrm{k} 145^{\circ} \mathrm{C} 5 \mathrm{~m}$ & & \\
$5 \mathrm{k} 140^{\circ} \mathrm{C} 5 \mathrm{~m}$ & & \\
& & \\
\hline
\end{tabular}

Table 11: List of bis-PCBM/PS samples and which reflectometers they were measured at.

Assessing the level of lateral inhomogeneities on bis-PCBM/PS bilayers

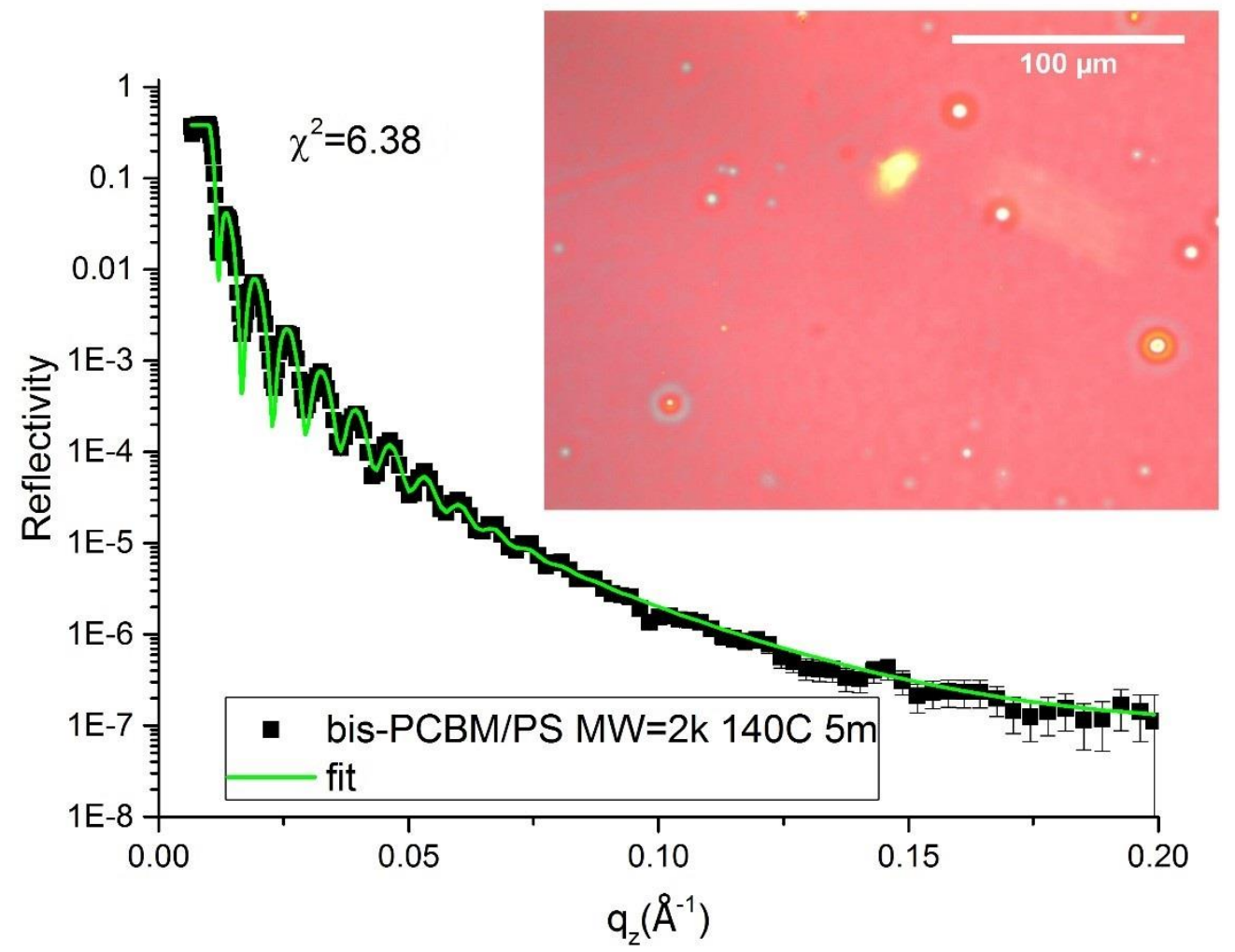

Figure 4.2-18: Micrograph and NR curve for annealed bilayer showing the lateral inhomogeneities on the sample. 


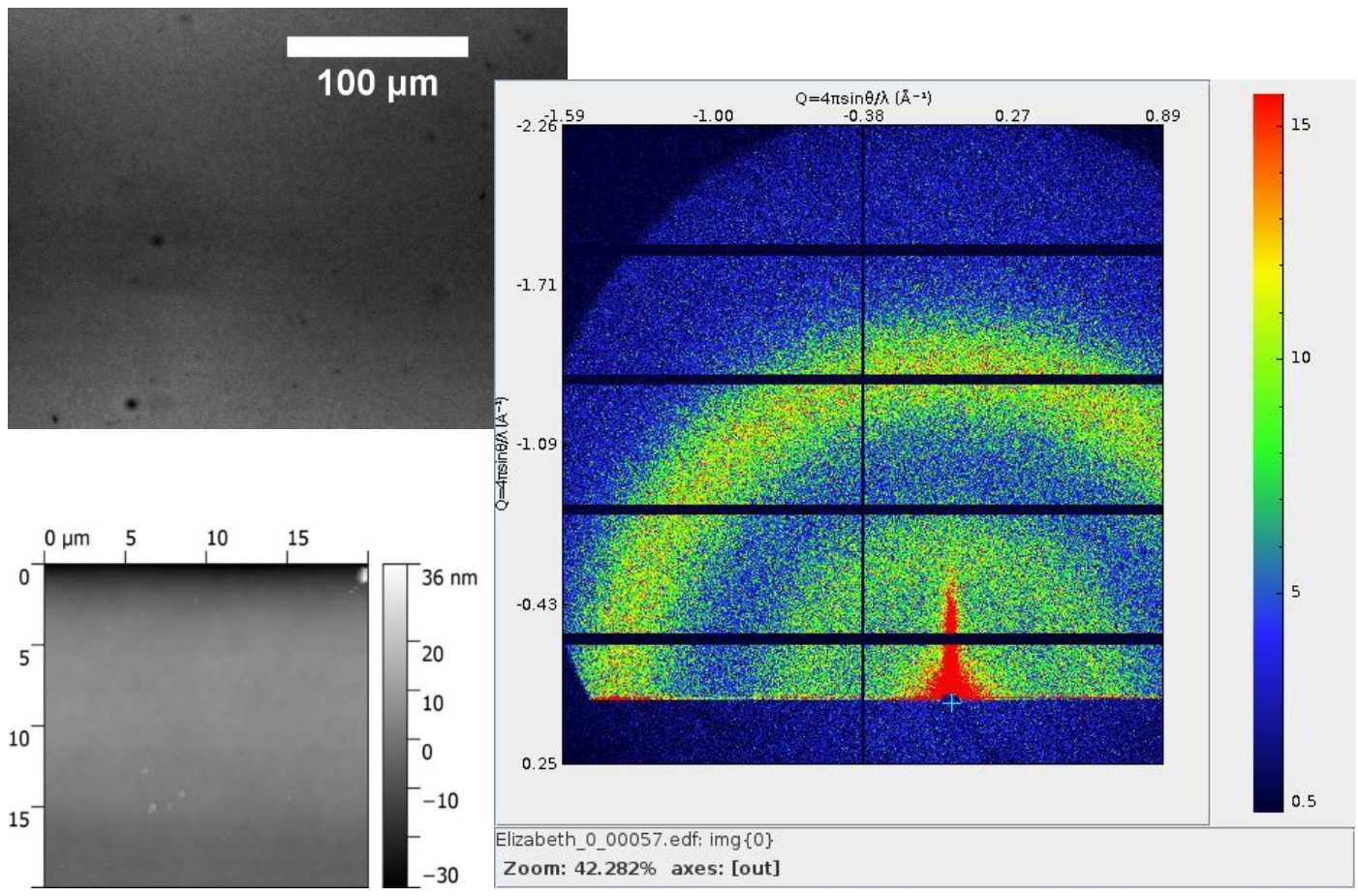

Figure 4.2-19: Images of a bis-PCBM/PS MW=100kDa bilayer annealed at 180C 5m. Top left: micrograph, bottom left: AFM image, bottom right: GIXD image.

Figure 4.2-18 and Figure 4.2-19 show optical micrographs, AFM images and GIXD maps from two (ex-situ annealed) bis-PCBM/PS samples, showing no indication of the presence of extensive crystallisation, as would be seen by GIXD. The defects do not appear to be contributing significantly to the measured reflectivity, which is dominated by the uniform regions of the sample. There is good agreement between parameters of the lower and the higher defect density films. The lateral inhomogeneities in bis-PCBM/PS are unlikely to be due to the formation of any fullerene crystals, and it is more likely that they arise from dewetting or other defects around contaminants. They are less extensive than in PCBM/PS. The magnitude of the effect on the SLD profile with MW is larger here than in PCBM/PS and therefore the results are likely to be even less sensitive to the presence of lateral inhomogeneities.

For one bis-PCBM/PS sample $\left(5 \mathrm{k}, 140^{\circ} \mathrm{C}\right)$ we see macroscopic differences across the sample, with some areas being very uniform and other areas (several mm away) containing significant number of defects/inhomogeneities (see figure 2-20) However there is still a nice fit for this sample, and the SLD profile is largely unaffected by this macroscopic variability across the sample. While some areas of this sample look 
rough and contain many defects, the changes seen at low MW for the bis-PCBM are more dramatic (in terms of the change in the SLD of the bottom layer of the sample) than for PCBM/PS. The impact of these macroscopic areas with larger roughness does not seem to affect the systematic behaviour with MW. Figure 4.2-20 shows the coverage of defects for different areas of this sample. 30 images were taken at random across the sample surface and the defect density was ranked as high, low and intermediate of which there were 3,14 and 13 respectively. Because the high and low defect density regions are separated macroscopically, the reflections from these different areas add up incoherently. The reflectivity from the rough $10 \%$ of the sample, is negligible with-respect-to the uniform areas of the sample, because of the low area coverage and the low reflectivity from these regions. 


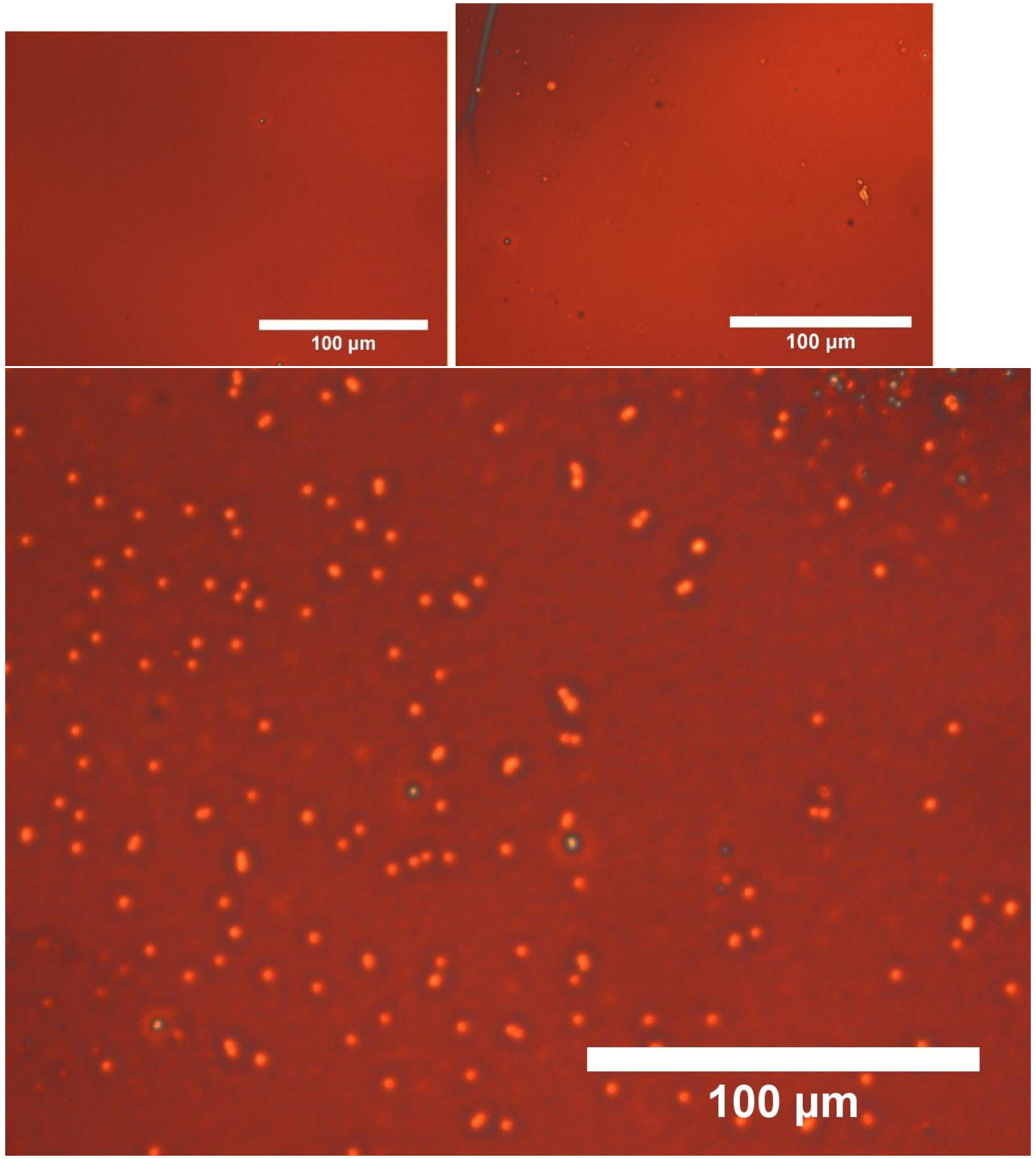

Figure 4.2-20: Micrographs showing variation in area coverage by defects for bisPCBM/PS 5k 140C 5m. Top left: a low defect density region, top right: intermediate defect density, bottom: high defect density.

\section{Film thickness}

\begin{tabular}{|l|l|l|}
\hline $\begin{array}{l}\text { Spinning speed }(\mathrm{krpm}) \text { and } \\
\text { concentration }(\mathrm{m} / \mathrm{m}) \text { of bis- } \\
\text { PCBM }\end{array}$ & $\begin{array}{l}\text { Thickness measured by AFM } \\
(\mathrm{nm})\end{array}$ & $\begin{array}{l}\text { Error of AFM measurement } \\
\text { (standard deviation) }(\mathrm{nm})\end{array}$ \\
\hline $2,2 \%$ & 41.8 & 10.2 \\
\hline
\end{tabular}




\subsection{The effect of different storage environments on annealed PCBM/PS composition profiles.}

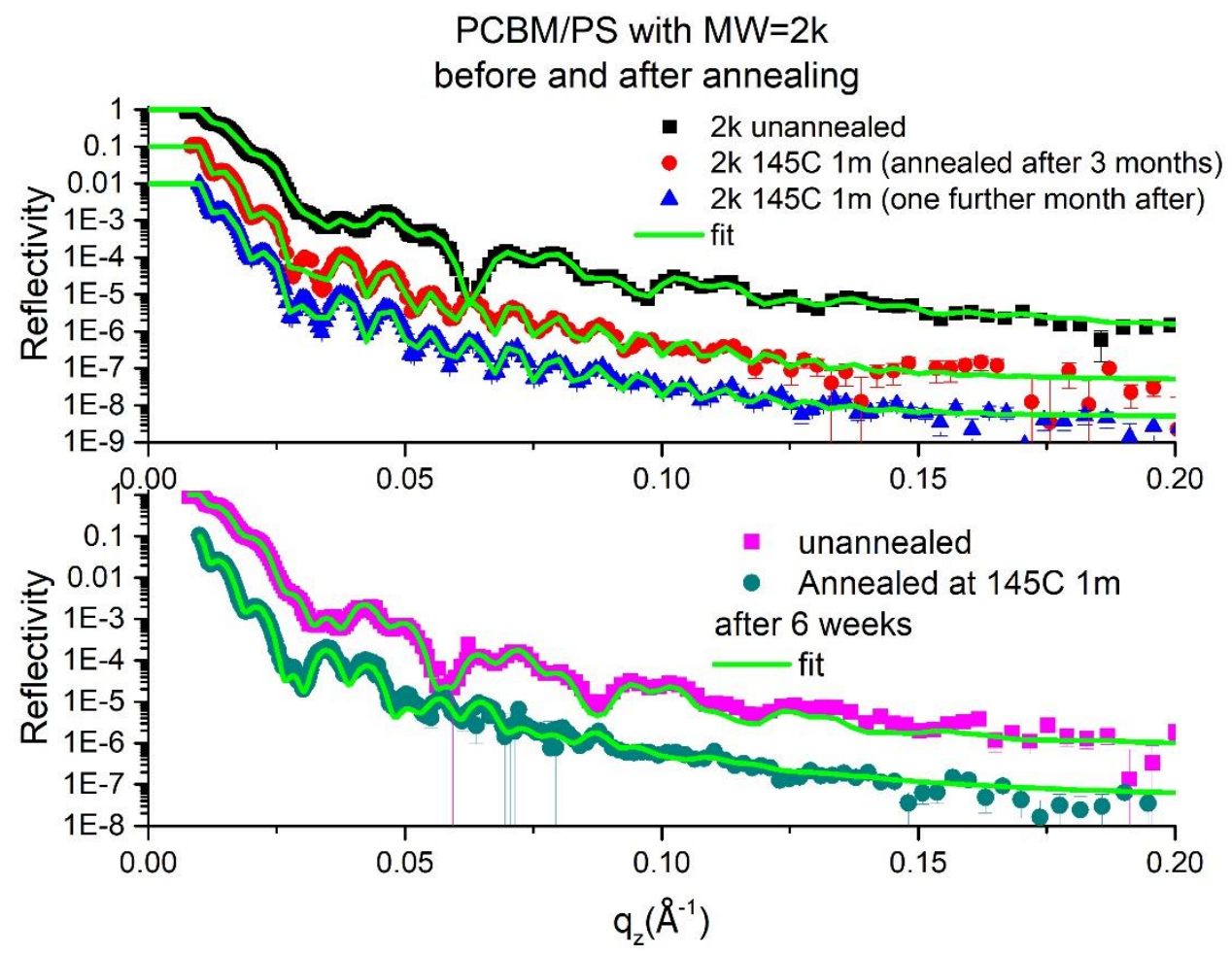

Figure 4.3-1: Reflectivity for two samples measured both prior to annealing and after annealing following storage. Both samples are PCBM/2k-PS.

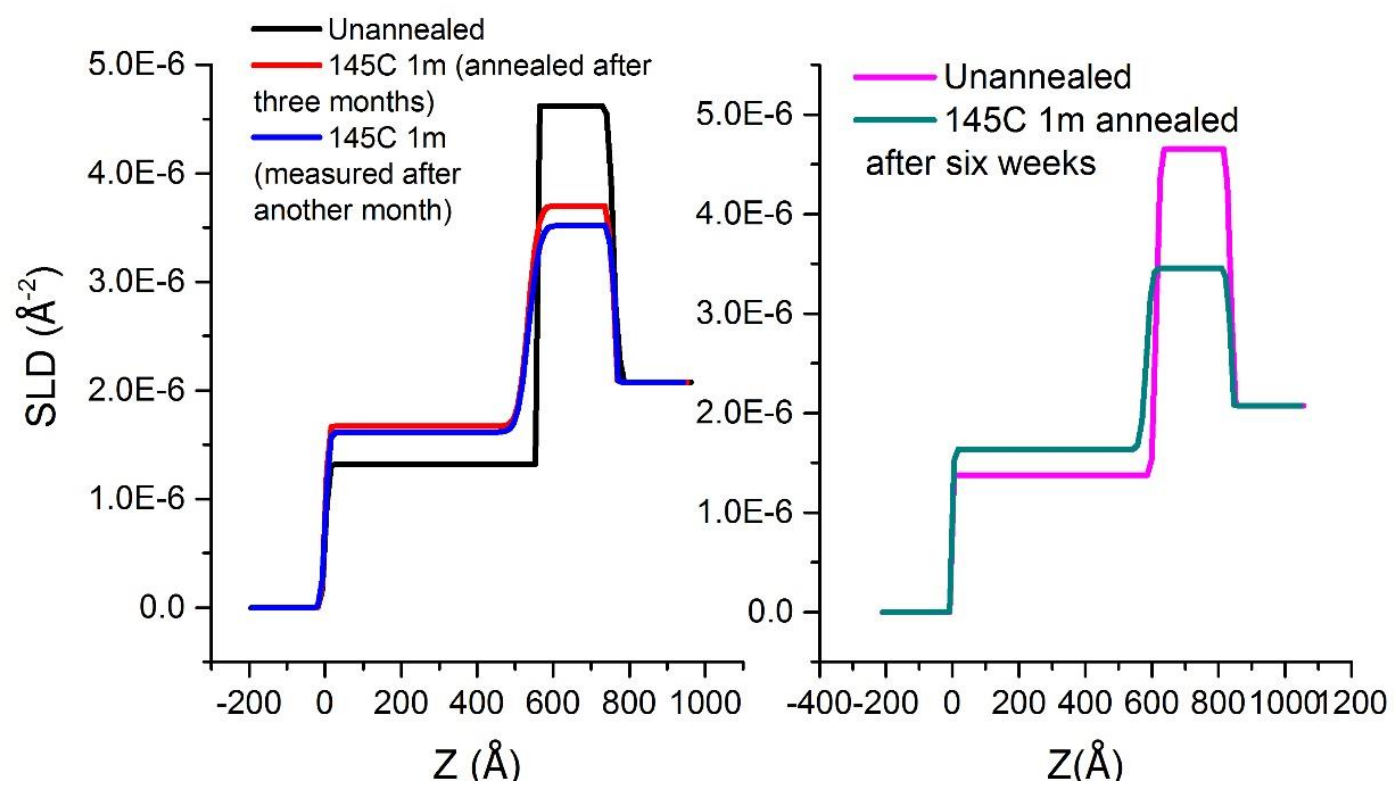

Figure 4.3-2: This figure shows the SLD profile for samples measured prior to annealing and after annealing three months later (left) and after six weeks (right). Typically the unannealed samples shown were measured within a week of fabrication. 
The initial motivation for studying the effect of storage/aging on PCBM/PS bilayers was an observation from two $2 \mathrm{kPS}$ samples which were left for several weeks prior to annealing (Figure 4.3-1 and Figure 4.3-2.). The samples were stored in air, in the dark, but were not stored under rigorously controlled conditions. Following annealing after this extended time (normally ex-situ samples were annealed after a few days and after no longer than a week) there was a significantly reduced SLD of the bottom layer of $3.6+/-0.1 \times 10^{-6} \AA^{-2}$, in comparison to samples that were annealed within a few days of fabrication. However the other behaviour, specifically the interfacial broadening and the elevated top layer SLD, was the same as that seen in Chapter 4 (although it may not represent the same composition if the species were chemically altered during aging).

To determine the cause of this observation, fresh samples were aged in different environments. There were five controlled conditions: i) aging in light and air (800 lux for one-week exposure on bilayers), ii) deliberately oxidised PCBM solution used to directly fabricate oxidised PCBM layers, iii) light exposure in an inert atmosphere (this results in oligomerisation as discussed in Chapter 2.2), iv) aging in the dark in air (covered in laboratory conditions at ambient temperature and atmosphere) and v) aging in the dark in an inert atmosphere (samples were left covered and in the glovebox). The changed chemistry due to oxidation or oligomerisation may affect the miscibility, the SLD and the interfacial roughness. Deliberately aged $2 \mathrm{k}$ samples are presented in this chapter, as well as $20 \mathrm{k}$ samples, to probe for MW dependence. The results for the oligomerised samples are shown in Section 4.3.2 below and the results for the other conditions are shown in Section 4.3.1. Oxidation of PCBM was assessed by Speller et. al using FTIR (E. M. Speller 2016).

As discussed in the introduction, it is known that fullerenes can change chemically in different ways, in response to illumination with visible light (oligomerisation and oxidation), and that it is also known that polymers can 'age' as they evolve towards equilibrium when stored for extended periods (even at temperatures below Tg (Pye and Roth 2015)). Therefore, a broad range of samples were looked at to try to understand the origin of the observed SLD profiles in Figure 4.3-2. 


\subsubsection{Aged/oxidised PS/PCBM bilayer samples}

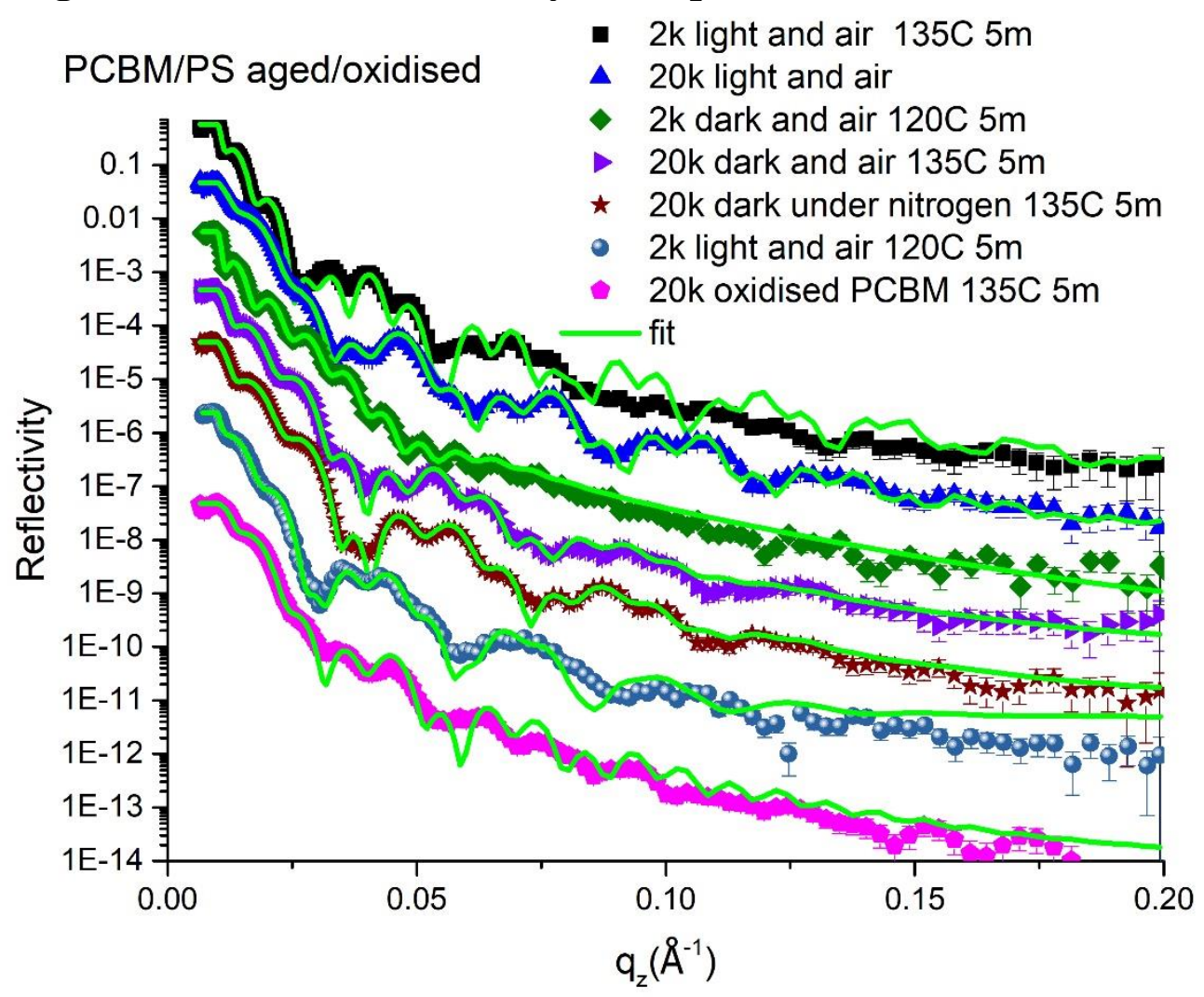

Figure 4.3-3: NR curves and fits for PCBM/PS samples aged under various conditions or made using oxidised PCBM. Bilayer fits with Gaussian interface roughness.

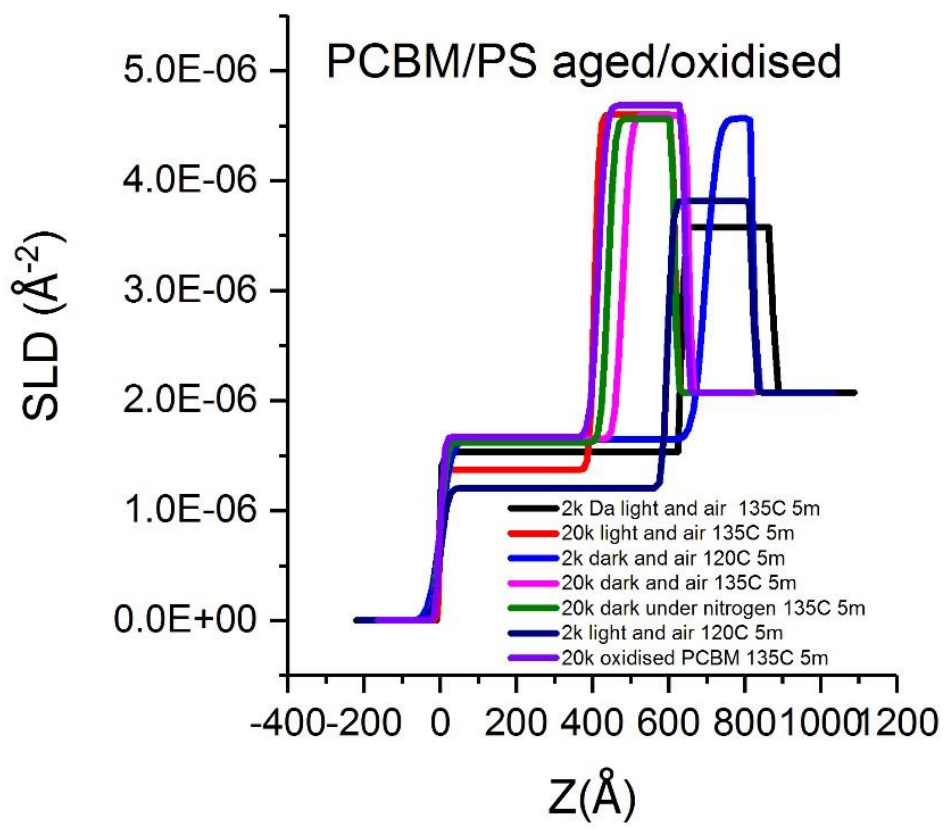

Figure 4.3-4: SLD bilayer profiles for PCBM/PS samples aged in various conditions or using oxidised PCBM and annealed at various times and using various molecular weight polystyrene ( $2 k$ and $20 k \mathrm{Da}$ ). 
Figuress 4.3-3 and 4.3-4 show the reflectivity curves, fits and SLD profiles for the aged/oxidised using bilayer models with Gaussian roughness at the buried interface and sample surface. In Figure 4.3-4 we see that the SLD of the bottom layer (PCBM rich) has lowered significantly for the two $2 \mathrm{k}$ PS samples aged in light and air compared to the values of the bottom layer measured in chapter 4.1 and compared to the other samples. These two illuminated samples were exposed to visible light at 800 Lux for one week using a lightbox containing a series of fluorescent lamps with reflectors, Osram L18W/827. This is the same effect we observed for the samples left in uncontrolled conditions for 3 months. This effect is only observed for the PS $\mathrm{MW}=2 \mathrm{k}$ Da samples. It is not evident in the 20k Da samples aged in the same conditions. Careful examination of the reflectivity curves themselves in Figures 4.31 and 4.3-3 shows that this significantly lower SLD value is not due to some minor subtlety in the fitting, but is a clear finding, associated with the steeper fall-off in reflectivity between the critical edge and $\mathrm{q} \sim 0.03 \AA^{-1}$, that is evident in these samples in comparison to the other samples in figure 4.3-3. This more rapid reduction in reflectivity with $\mathrm{q}$, is a clear result of lower contrast at the buried interface.

The 'oxidised' sample in figures 4.3-3 and 4.3-4 was fabricated by illuminating a $2.5 \%$ (by weight) PCBM solution in chlorobenzene for 70 hours with AM1.5 G spectrum (which represents the overall yearly average for mid-latitudes at 1.5 atmospheric thicknesses), under a Newport 92193A-1000 solar simulator, for one week (E. M. Speller 2016; Emily M. Speller et al. 2017; Z. Li et al. 2013). The 'oxidised' sample exposed to a solar simulator would be expected to have significantly more oxidation of the PCBM molecules, in comparison to the samples illuminated in the lightbox (which would be expected to have low levels of oxidation). However, in common with the other 20k PS samples, this sample does not show any evidence of a drastically different SLD profile, with a significantly lower bottom layer SLD, in comparison to the 'normal' samples in chapter 4.1. This emphasises the significance of the polymer MW in terms of the observation of different mixing behaviour between PS and oxidised PCBM molecules.

\subsubsection{Oligomerised PCBM bilayer samples}

Oligomerisation was performed by subjecting the PCBM single layers to light exposure under an inert atmosphere as described in Section 2. Oligomerisation was 
then controllably reversed from a maximum oligomerisation of $40 \%$ ( $\mathrm{Z}$. Li et al. 2013), by heating under vacuum in the dark at different temperatures for 1 hour, as described in Wong et al. (Wong et al. 2014). Heating at temperatures of $110^{\circ} \mathrm{C}, 130$ ${ }^{\circ} \mathrm{C}$ and $160{ }^{\circ} \mathrm{C}$ for one hour gave oligomerisation percentages of approximately $25 \%, 10 \%$, and $0 \%$ respectively. Bilayers were then fabricated in the way described in section 3.2 by adding PS layers of either $2 \mathrm{k}$ or $20 \mathrm{k}$. The bilayers were then annealed for 5 minutes at $135 \quad{ }^{\circ} \mathrm{C}$ temperatures.

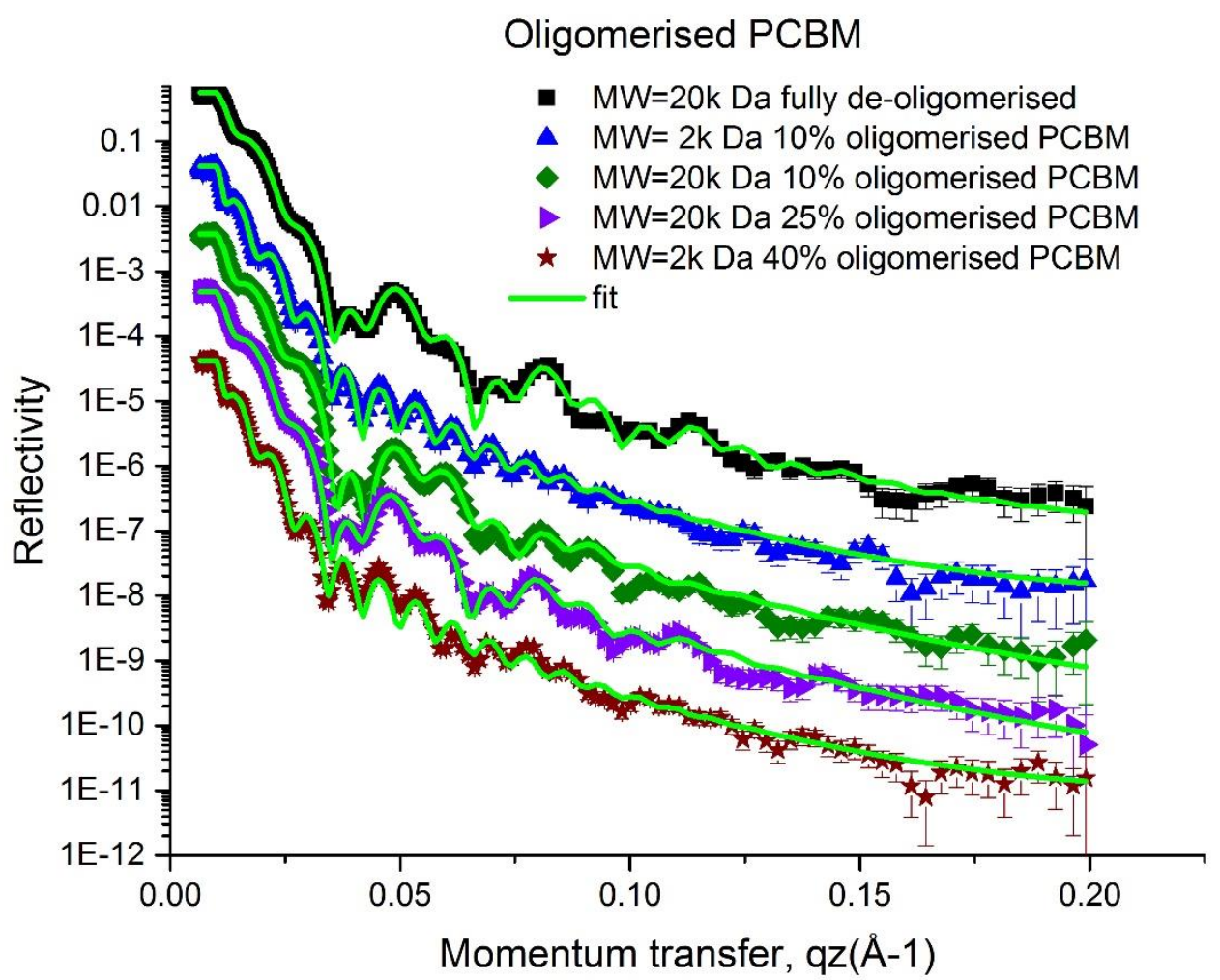

Figure 4.3-5: NR curves and fits for samples whose PCBM layer was oligomerised and de-oligomerised to varying extents by thermal annealing before deposition of the top PS layer with varying PS MW using a bilayer model. 40\% was maximally oligomerised and not annealed prior to deposition of top layer, 25\% was annealed at $110^{\circ} \mathrm{C} 1 \mathrm{hr}, 10 \%$ was annealed at $130^{\circ} \mathrm{C} 1 \mathrm{hr}$, fully de-oligomerised was annealed at $160^{\circ} \mathrm{C} 1 \mathrm{hr}$. Following deposition of top layer all samples were annealed at $135^{\circ} \mathrm{C}$ for 5 mins. 


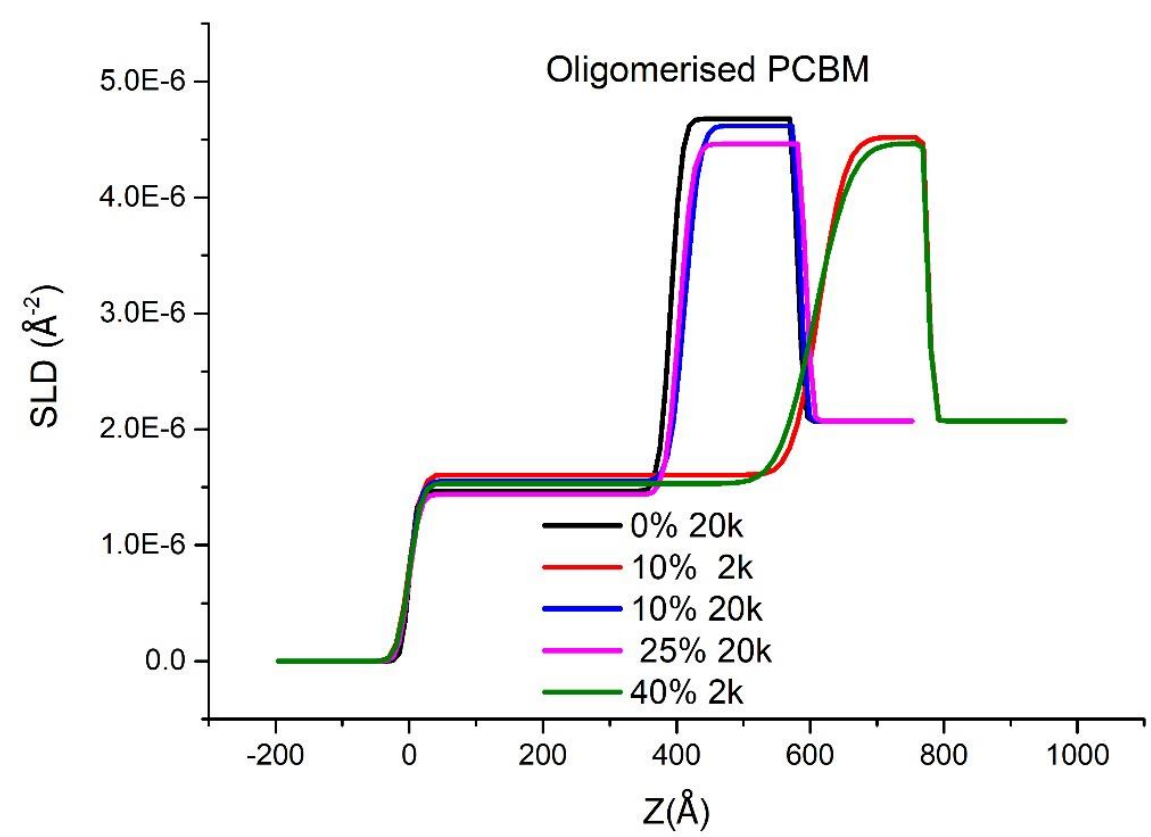

Figure 4.3-6: SLD profiles for samples whose PCBM layer was oligomerised and deoligomerised to varying extents by thermal annealing before deposition of the top PS layer with varying PS MW.

As can be seen in Figure 4.3-6, oligomerisation didn't change any of our observations in relation to previous results in Chapter 4.1 with the interfacial width and elevated SLD of the top layer (which indicates mixing) being in the range we would expect from unaltered PCBM. As in chapter 4.1, the broader interfacial roughness for the $2 \mathrm{k}$ samples is evident in Figure 4.3-6. As there is no observed drop in the SLD of the bottom layer for any of the oligomerised samples (including the $2 \mathrm{k}$ PS samples) we can conclude that this known phenomenon is not responsible for the lowered SLD of the bottom (PCBM-rich) layer shown in Figure 4.3-2. The 40\% oligomerised sample fit in Figure 4.3-5 does not quite capture the fringes in the data, as well as in the other samples, so it was fitted with multiple thin layers with no roughness between them (a multilayer fit). There are also some aspects of the fits in Figure 4.3-3 that also do not account for the data, so some of these curves have been fitted with a multilayer fit in Section 4.3.3 below. 


\subsubsection{Multilayer fits}

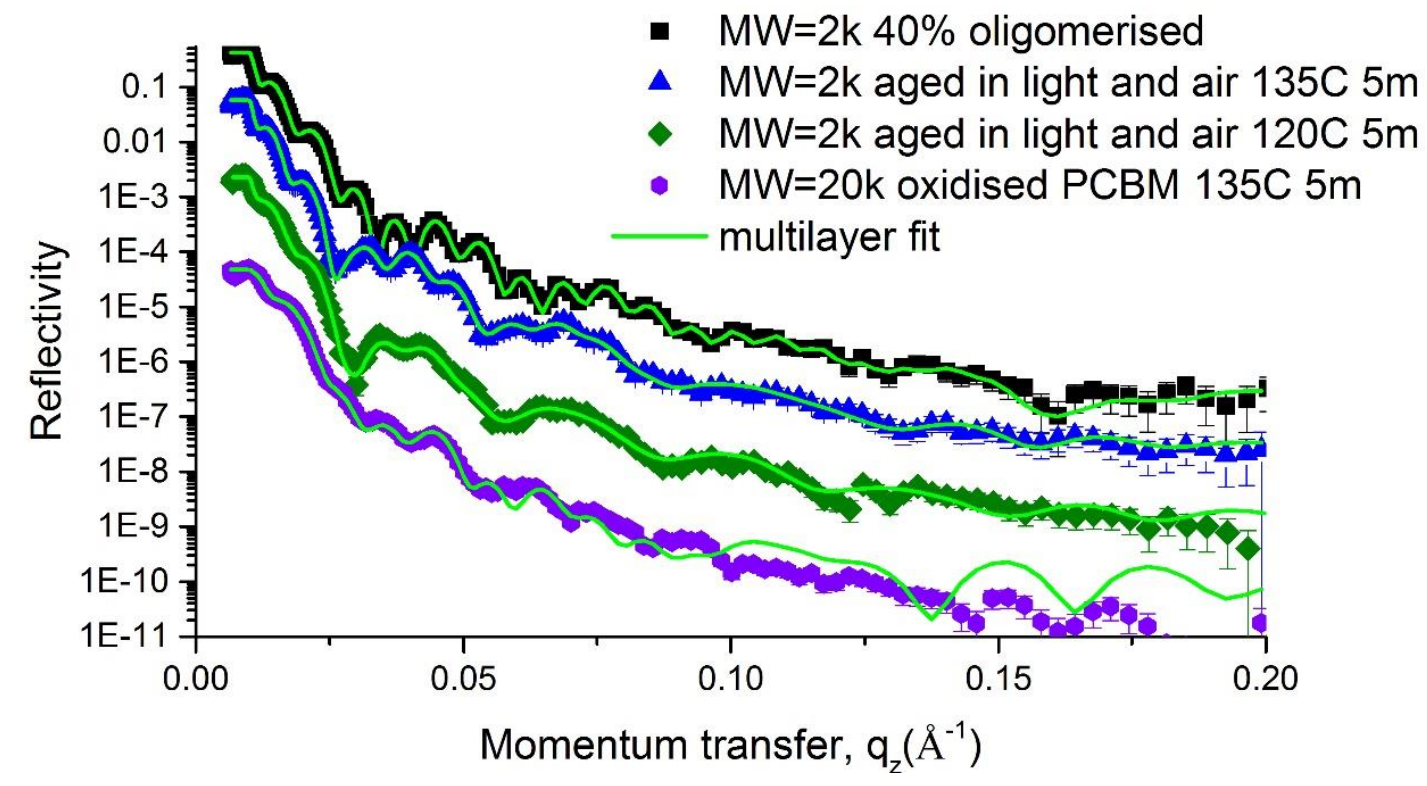

Figure 4.3-7: Multilayer fits with no roughness between the layers.

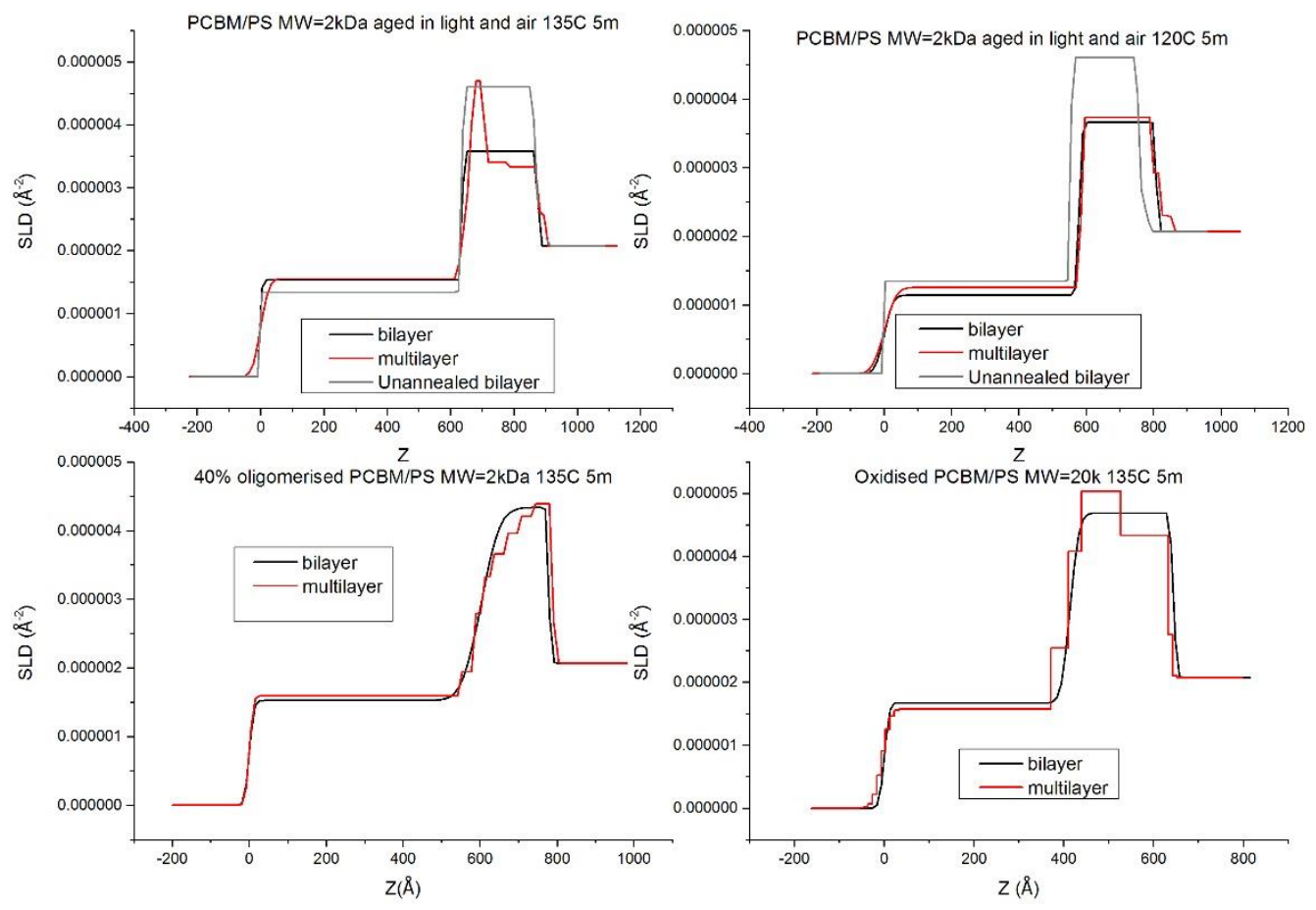

Figure 4.3-8: Comparison of bilayer fit with roughness between the layers and multilayer fit with no roughness between the layers. The unannealed data is estimated from NR measurements on unannealed bilayers. 
To attain a better fit to the samples in Figure 4.3-7 a multilayer fitting procedure was used with no roughness between the layers in order to better understand the composition profile. In Figure 4.3-8 the results of this multilayer fitting procedure show relatively good agreement with the bilayer fits in terms of thicknesses and the mean SLD of each layer. In all four cases, however, the multilayer fits are significantly better than the bilayer fits. The chi-squared values for the $2 \mathrm{k}$ aged in light and air $135^{\circ} \mathrm{C} 5 \mathrm{~m}$ were 21.8 for the bilayer and 2.45 for the multilayer. For the $2 \mathrm{k}$ aged in light and air the chi-squared value was 6.84 for the bilayer and 2.6 for the multilayer. For the $2 \mathrm{k} 40 \%$ oligomerised sample $135^{\circ} \mathrm{C} 5 \mathrm{~m}$ the chi-squared was for the bilayer 5.8 and 3.9 for the multilayer. The oxidised PCBM $20 \mathrm{k}$ sample $135^{\circ} \mathrm{C} 5 \mathrm{~m}$ had chi-squared of 21.9 for the bilayer and 9.4 for the multilayer.

The two 2k PS samples aged in light and air, still show a greatly reduced SLD in the bottom layer in comparison to pure PCBM. There is some non-uniformity in the SLD of the bottom layer region of the sample annealed at $135{ }^{\circ} \mathrm{C}$ tentatively suggesting some segregation of the PCBM to the interface. This is not seen in the similar sample annealed at 120C. However, since there are only two samples, it is not possible to assess the repeatability of this rather subtle difference and see whether there is a genuine temperature dependence on the composition profile. What is clear though is that these two 2k PS samples that have been aged in light and air both have very different SLD profiles from the 'standard' samples reported in chapter 4.1, and also from the 20k PS sample aged under the same conditions (shown in Figure 4.3-4). It is also worth pointing that the extensively oxidised PCBM/20k PS sample in Figure 4.3-7 and Figure 4.3-8, while not being as wellfitted as the other samples, does not show any evidence of a significantly lower SLD bottom layer.

The multilayer fit to the $40 \%$ oligomerised sample features a 'broader interface', which approaches the thickness of the bottom layer. This could explain the poor fit using a Gaussian interface between two uniform layers if the interface approaches either layer thickness. The bilayer and multilayer fits are, however, quite similar and we see no reduction in the bottom layer SLD in this sample. 


\subsubsection{Interfacial roughness and top layer SLD of aged samples}

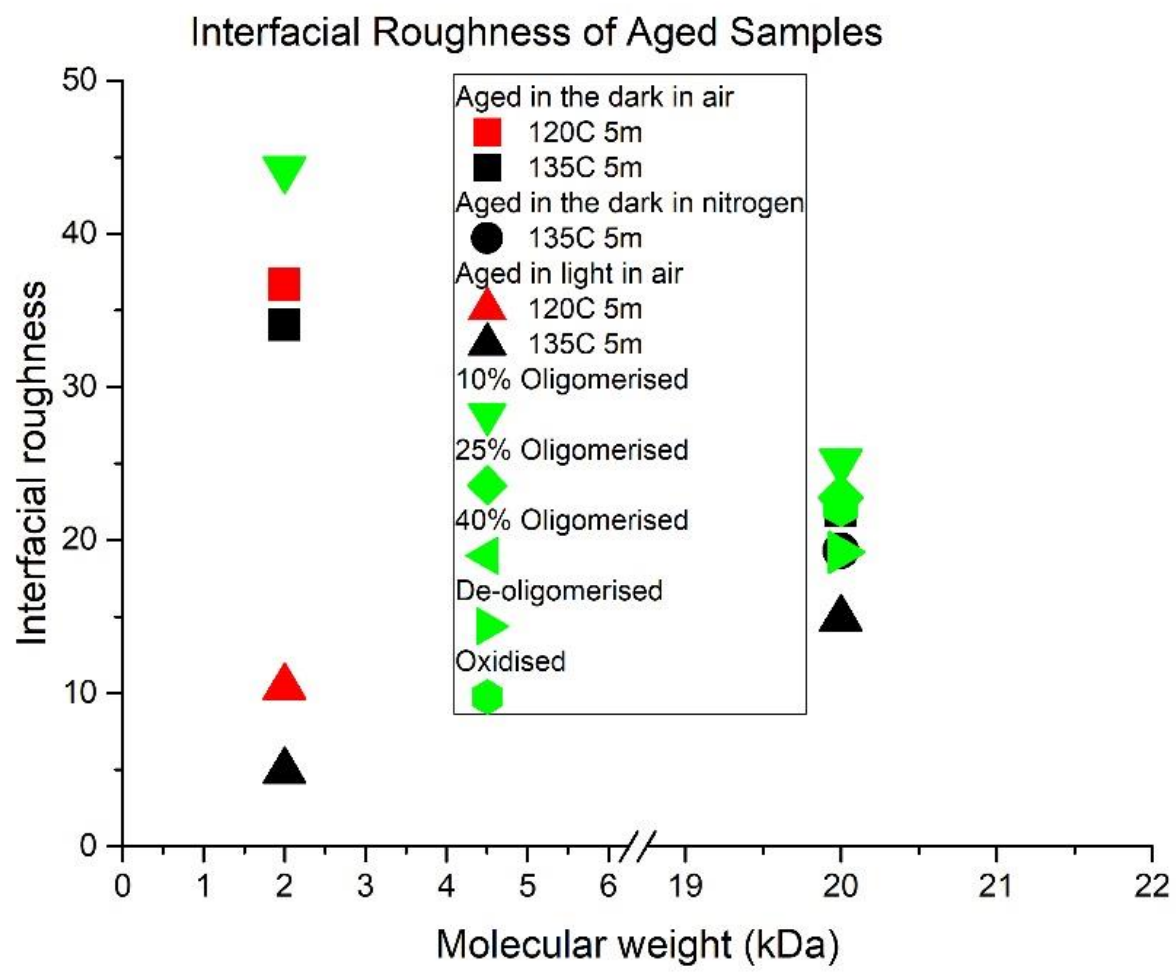

Figure 4.3-9: Interfacial roughness from bilayer fits as a function of molecular weight for samples deliberately aged variously in light, dark, air and under nitrogen. The samples aged in light was for one week at 800 lux in air and in the dark for 6 weeks.

In Figure 4.3-9, values for the interfacial roughness for the aged samples are plotted. Here, there is the same overall trend observed in chapter 4.1 with broader interfaces for the lower MW, for all samples except the two 2k PS samples aged in light and air. The bilayer fits for these two samples seem to have a sharper interface than both the $2 \mathrm{k}$ PS samples in chapter 4.1, and the 20k samples, although caution needs to be exercised with regard to the meaning of the interface roughness parameter here, in the light of the quality of the bilayer fits in comparison to the multilayer fits for these two samples. Figure 4.3-10 shows the top layer SLD for the aged samples. The data is very similar to that of chapter4.1, suggesting that the diffusion of PCBM into the top layer occurs as before. However, there is a low value for the top layer of the PS $2 \mathrm{k}$ sample aged in light and air annealed at 120C indicating perhaps some temperature dependence of the Flory-Huggins chi parameter. There is also a lower top layer SLD for some 20k samples. 


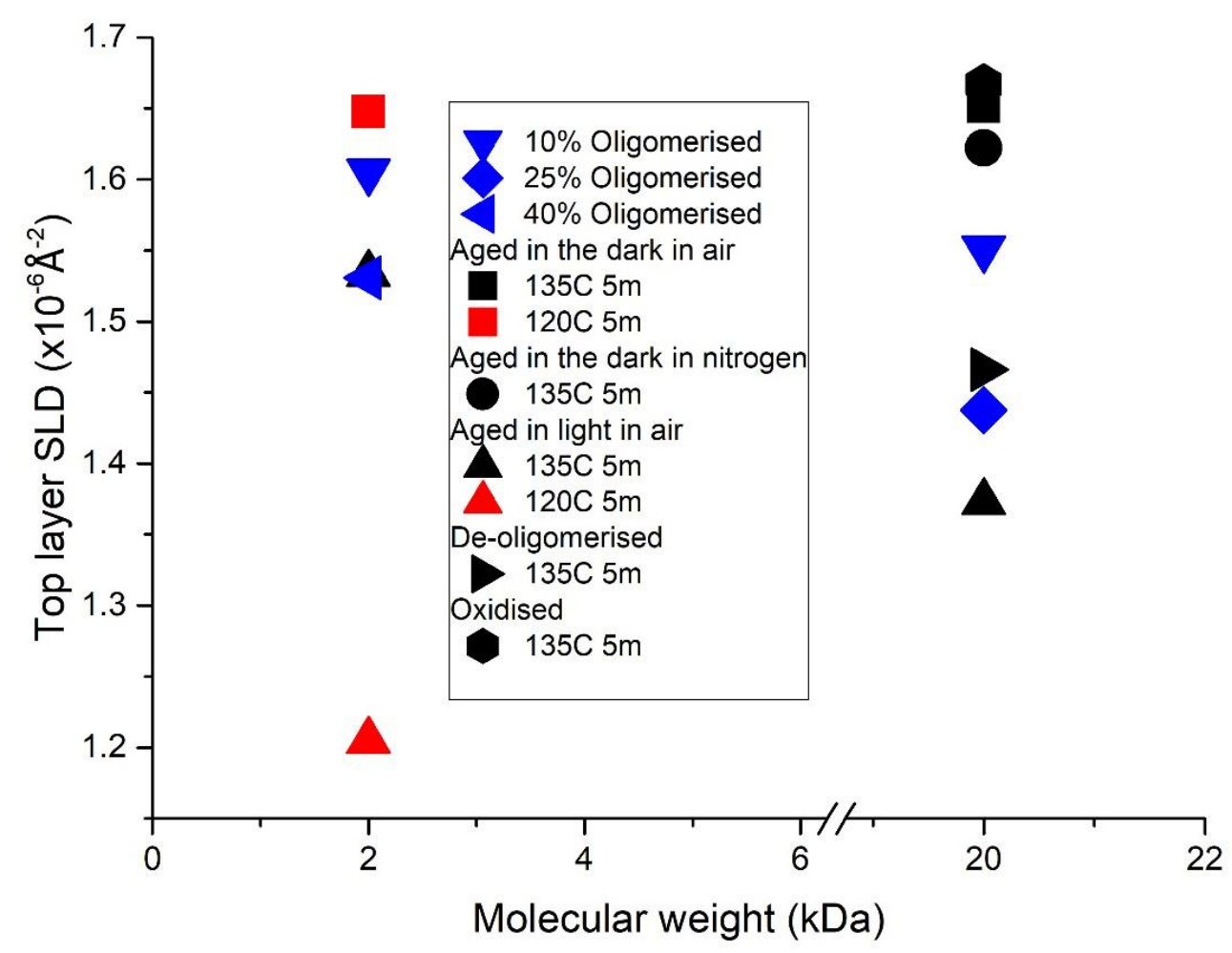

Figure 4.3-10: Top layer SLD as a function of molecular weight for samples deliberately aged variously in light, dark, air and under nitrogen. The samples aged in light was for one week at 800 lux in air, and in the dark for 6 weeks.

These results suggest that light exposure should be monitored during the fabrication of polymer-fullerene bilayers and that oligomerisation does not have a strong effect on the composition profiles except for the largest extent of oligomerisation looked at $(40 \%)$.

In summary there are no major differences between the SLD profiles of the 'standard' samples reported in chapter 4.1 and the SLD profiles of any of the oligomerised samples or the aged/oxidised samples, except for the two $2 \mathrm{k}$ PS samples aged in light and air. This conclusion is robust with-respect-to the use of both a simple bilayer model and a multilayer model. The fact that samples with $20 \mathrm{k}$ PS, or those aged in the dark (20k PS and 2k PS, in air or under nitrogen) do not show any significant difference in comparison to the 'standard' samples, and that significantly lower bottom layer SLDs are only observed for the two 2k PS bilayers aged under light in air, is a strong indication that light-induced oxidative changes to the PCBM chemical structure are the cause of these differences. The PS MWdependence of the behaviour is significant. The fact that both the 20k PS sample, that 
is subjected to the same illumination conditions as these two 2k PS samples, and the extensively oxidised PCBM sample (again using 20k PS) do not show any significant differences in SLD profile w.r.t. the standard samples, suggests that these light-induced oxidative changes are having a direct effect on the miscibility between this photo-chemically-degraded PCBM and PS. It is therefore plausible that the observed behaviour is a manifestation of different equilibrium thermodynamics in the degraded-PCBM/PS system (although no annealing time studies have been performed so far).

\subsubsection{Appendix}

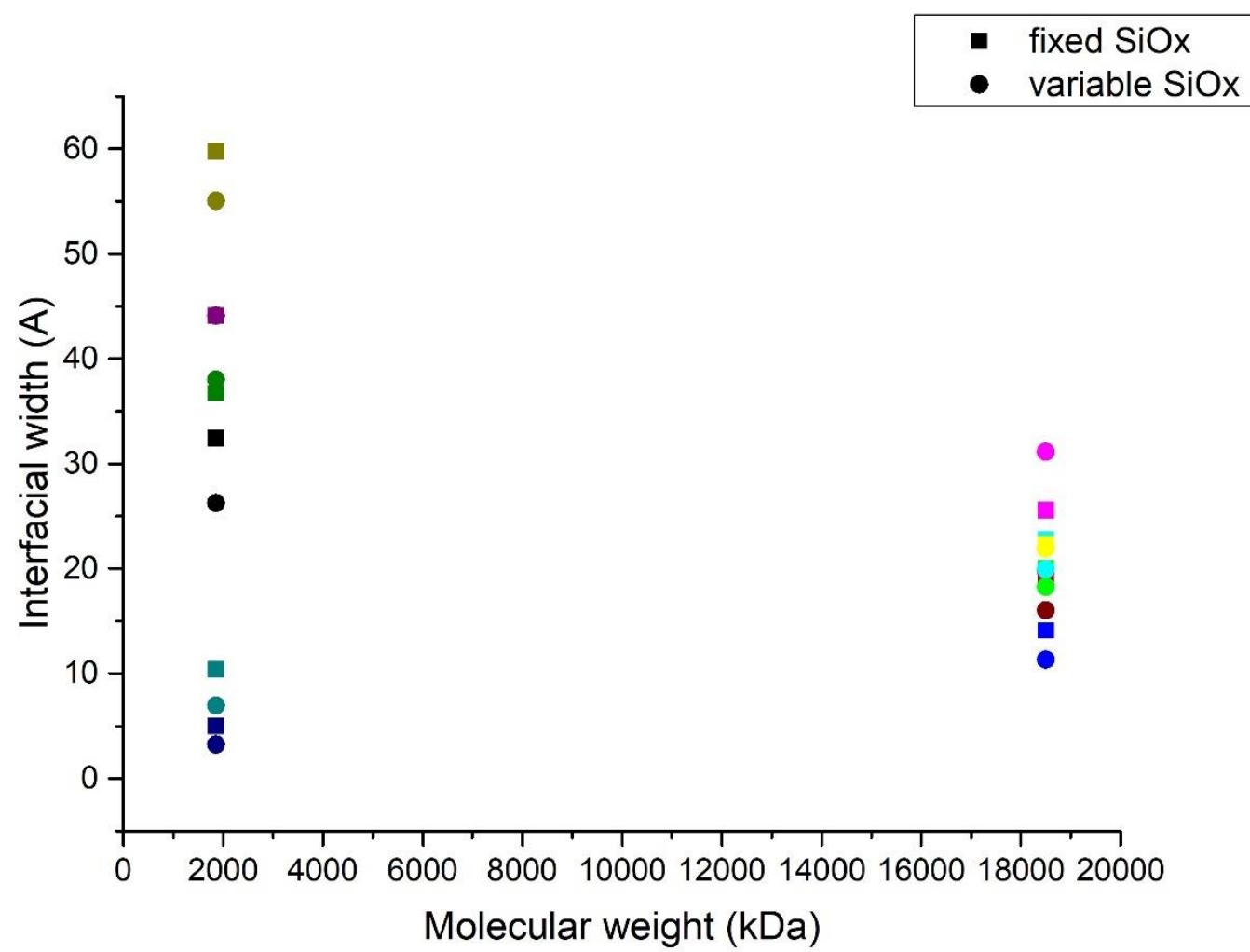

Figure 4.3-11: Parameter values for fixed and varied oxide layers. The same colour represents the same sample. 


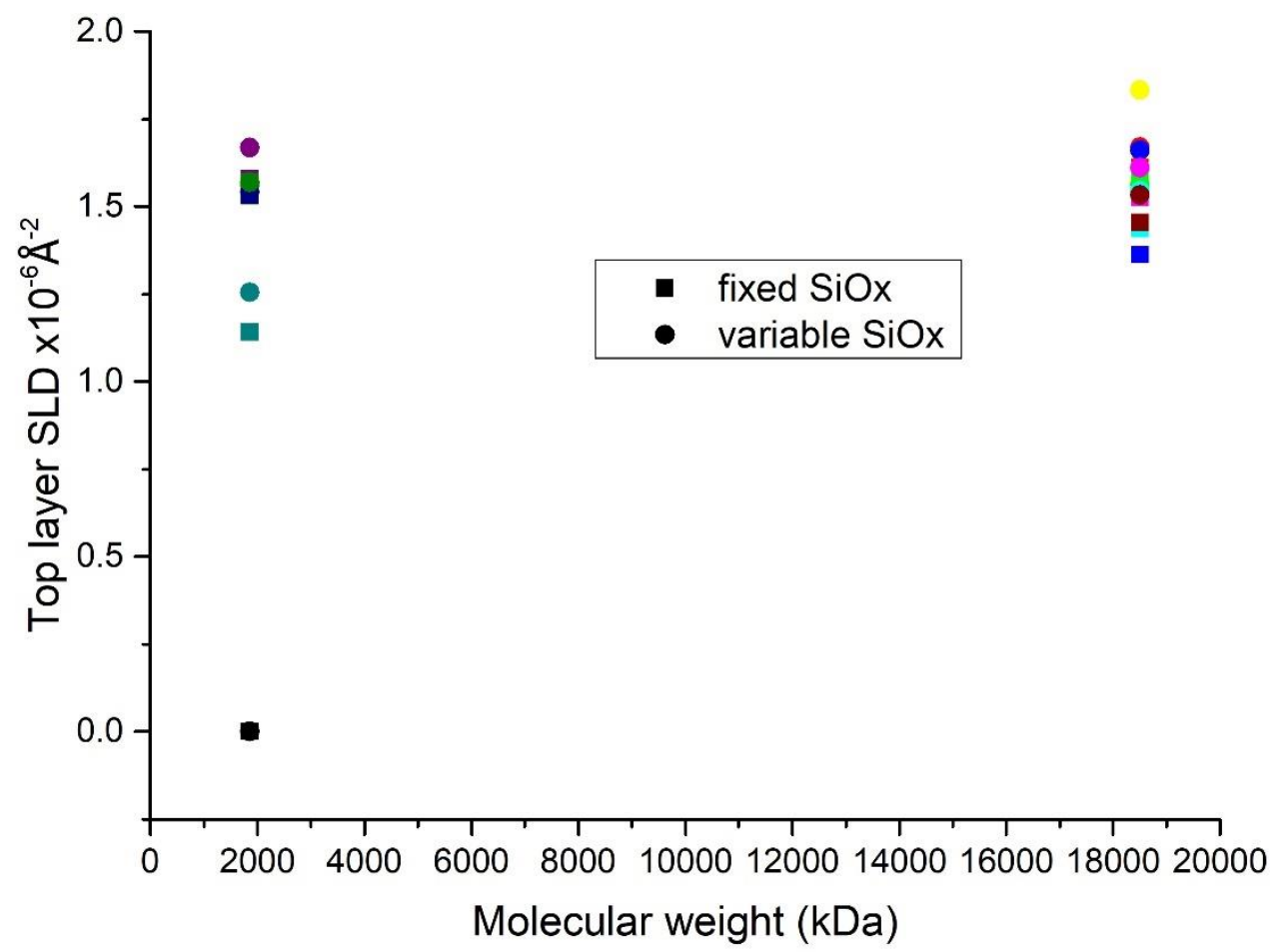

Figure 4.3-12: Parameter values for fixed and varied oxide layers. The same colour represents the same sample.

\section{Sample List}

Table 12 shows the NR sample list showing which samples were measured at which reflectometers.

\begin{tabular}{|l|l|l|}
\hline INTER & Figaro & D17 \\
\hline 20k $135^{\circ} \mathrm{C} 5 \mathrm{~m}$ aged: dark air & $2 \mathrm{k}$ unannealed & \\
20k $135^{\circ} \mathrm{C} 5 \mathrm{~m}$ aged: dark nitrogen & $2 \mathrm{k} 145^{\circ} \mathrm{C} 1 \mathrm{~m}$ (three months & \\
20k $135^{\circ} \mathrm{C} 5 \mathrm{~m}$ aged: light air & later) & \\
$20 \mathrm{k} 135^{\circ} \mathrm{C} 5 \mathrm{~m}$ oligomerised $(110 \mathrm{C} 1 \mathrm{hr}) 25 \%$ & $2 \mathrm{k}$ unannealed & \\
$20 \mathrm{k} 135^{\circ} \mathrm{C} 5 \mathrm{~m}$ oligomerised $(130 \mathrm{C} 1 \mathrm{hr}) 10 \%$ & $2 \mathrm{k} 145^{\circ} \mathrm{C} 1 \mathrm{~m}$ & \\
$20 \mathrm{k} 135^{\circ} \mathrm{C} 5 \mathrm{~m}$ oxidised & & \\
$2 \mathrm{k} 135^{\circ} \mathrm{C} 5 \mathrm{~m}$ oligomerised(-)40\% & & \\
$2 \mathrm{k} 135^{\circ} \mathrm{C} 5 \mathrm{~m}$ aged: light air & & \\
$2 \mathrm{k} 135^{\circ} \mathrm{C} 5 \mathrm{~m}$ oligomerised $(130 \mathrm{C} 1 \mathrm{hr}) 10 \%$ & & \\
$20 \mathrm{k} 135^{\circ} \mathrm{C} \mathrm{5m}$ oligomerised(160C $\left.1 \mathrm{hr}\right) 0 \%$ & & \\
$2 \mathrm{k} 120^{\circ} \mathrm{C} 5 \mathrm{~m}$ aged: dark air & & \\
$2 \mathrm{k} 120^{\circ} \mathrm{C} \mathrm{5m}$ aged: light air & & \\
& & \\
\hline
\end{tabular}

Table 12:List of where the stated samples were measured. 
Assessing the level of lateral inhomogeneities

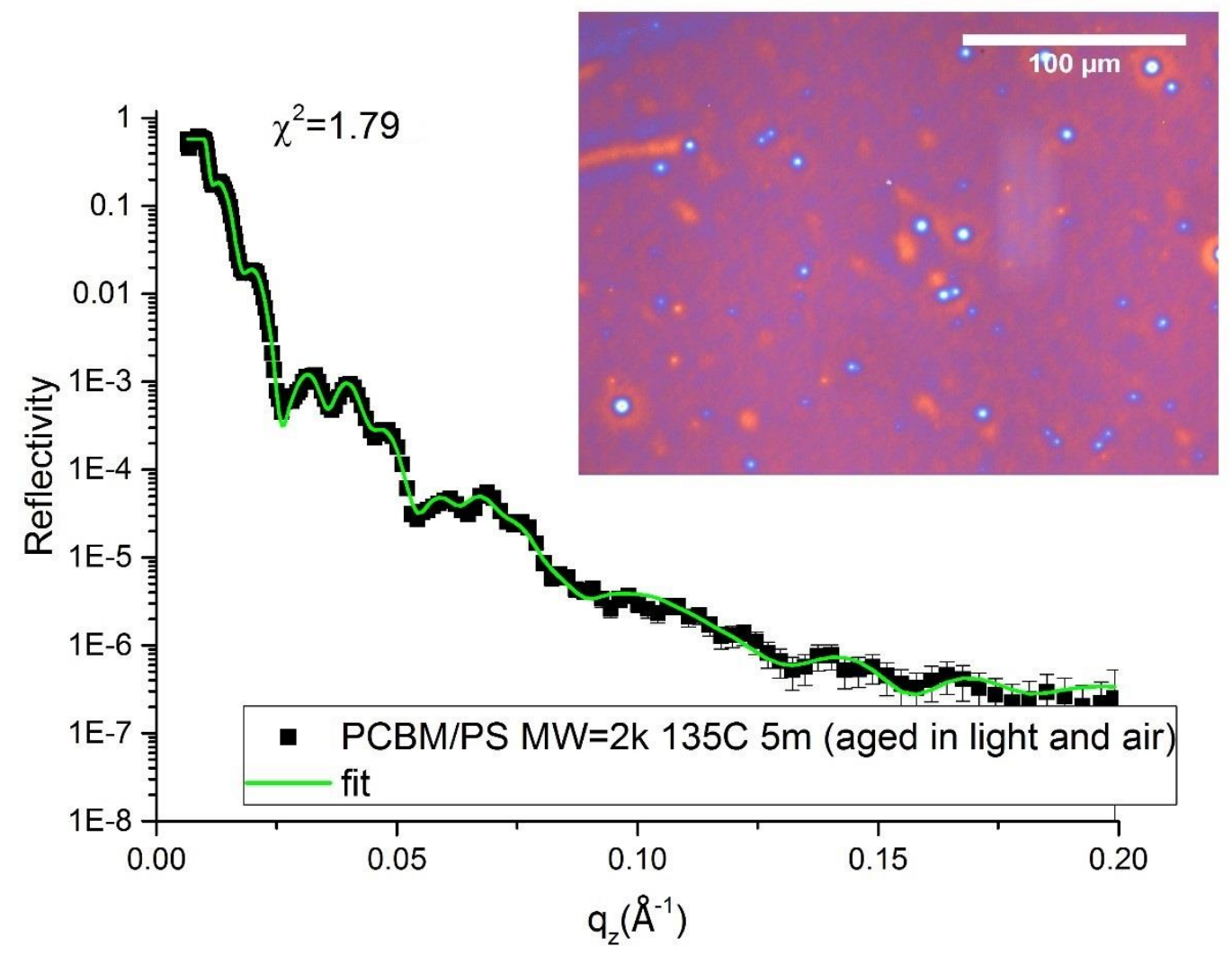

Figure 4.3-13: Micrograph and NR curve for annealed bilayer showing the lateral inhomogeneities on the sample.

Figure 4.3-13 shows the micrograph and NR curve for PCBM/PS $2 \mathrm{k} 135 \mathrm{C} 5 \mathrm{~m}$ samples aged in light and air. This sample shows the presence of some defects. While the level of lateral inhomogeneities may in principle be one reason why the bilayer fit isn't great, in practise the level of these inhomogeneities is similar to many other samples that do give good bilayer fits. 


\subsection{Bilayers using a non-fullerene acceptor and a conjugated polymer}

In this section a conjugated polymer and non-fullerene acceptor are replaced in turn for the PS and PCBM layers in comparison to Chapter 4.1 where a PS/PCBM bilayer was looked at. These replacements were looked at to determine the feasibility of making bilayers with these materials to get an overview of the mixing behaviour after annealing. Both sets of samples were initially fabricated as bilayers with polymer on the top and fullerene or non-fulleren on the bottom. The initial motivation for these preliminary studies was to assses the feasibility of fabricating bilaers with these materials. In doing so, extensive evidence was found for mixing in both systems as in Fig 4.4-2 and 4.4-3 below. Conjugated polymers are more suitable to devices yet their large polydispersity giving them a broad range of MW makes them unsuitable for the type of detailed study performed in Chapter 4.1.

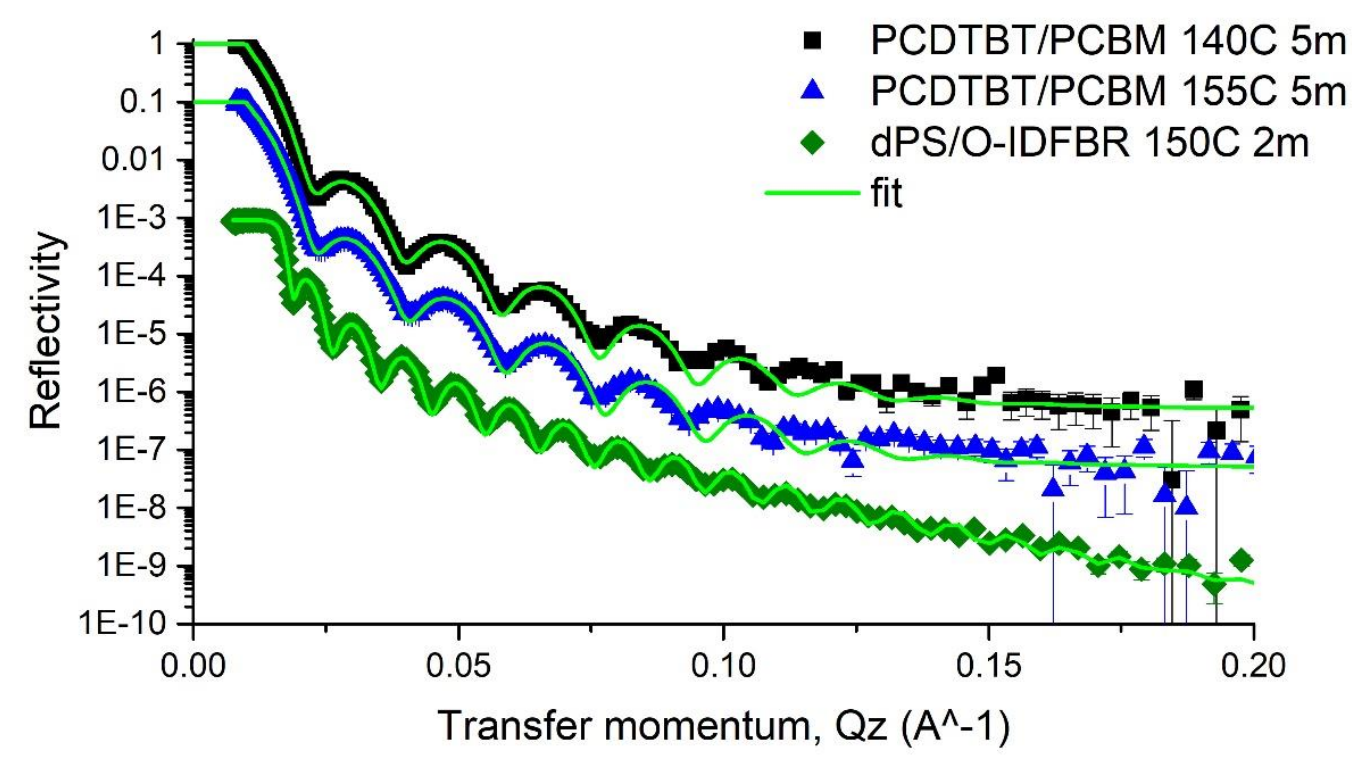

Figure 4.4-1: Reflectivity curves and fits for single layer blend (initially a bilayer) of PCBM and PCDTBT and a non-fullerene O-IFTBR deuterated PS bilayer.

Figure 4.4-1 to Figure 4.4-3 show the NR curves and SLD profiles for two other systems. The PCDTBT/PCBM samples were made according to the standard fabrication procedure as a bilayer. Following thermal annealing and NR experiments, it became apparent that the bilayers had merged via extensive mixing and the 
resultant composition profile is a single $300 \AA$ thick layer with an SLD of $3.2 \times 10^{-6} \AA^{-2}$.

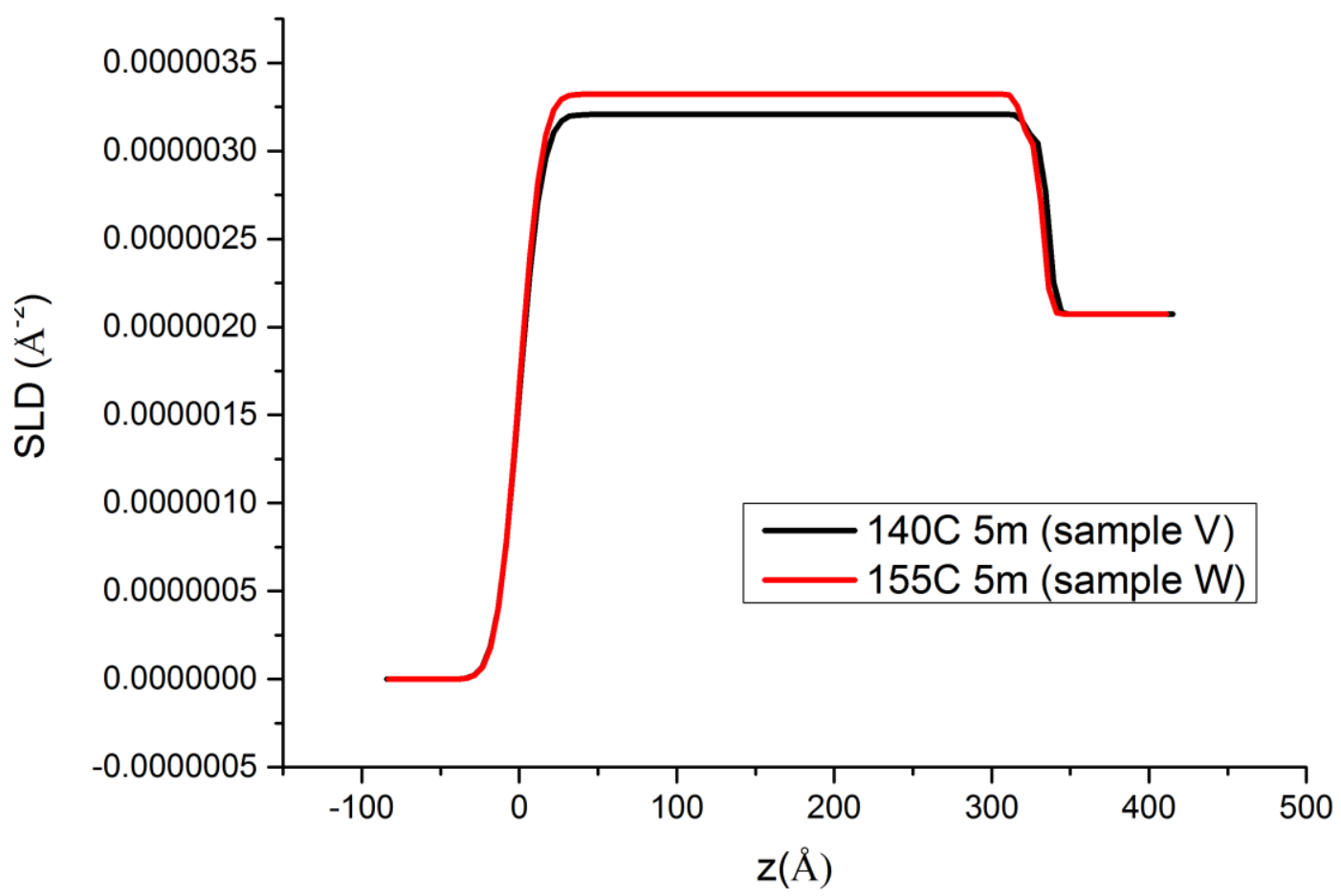

Figure 4.4-2: SLD profile for PCBM/PCDTBT blend. 


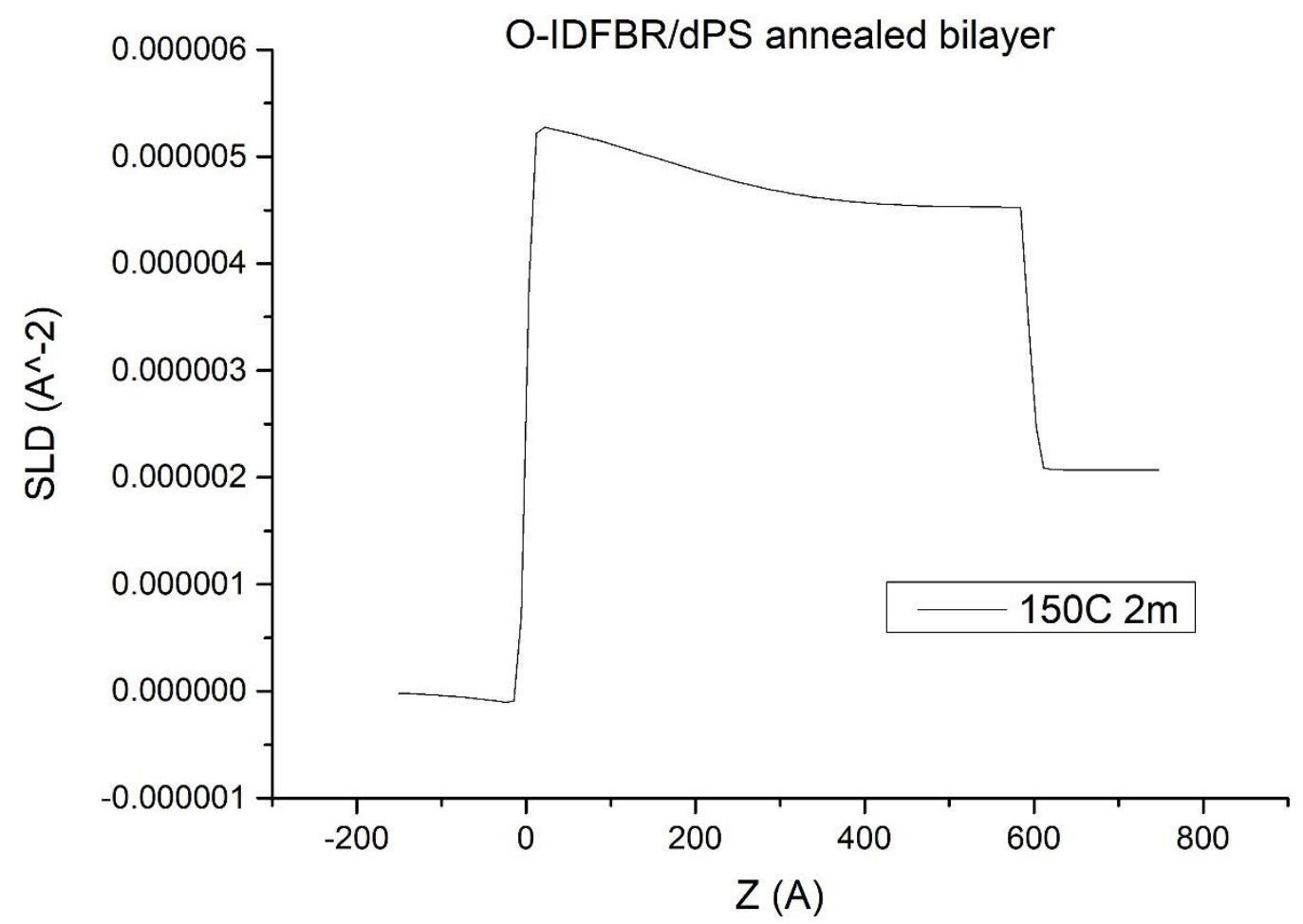

Figure 4.4-3: O-IDFBR/dPS bilayer SLD annealed above the glass transition.

The non-fullerene acceptor looked at, OIDFBR, was made into a bilayer with d-PS which has an SLD of $5.95 \times 10^{-6} \AA^{-2}$. Following annealing we obtained a bilayer with top layer SLD of $5.45 \times 10^{-6} \AA^{-2}$ and bottom layer SLD of $4.53 \times 10^{-6} \AA^{-2}$ with an interfacial roughness of $208 \AA$. This indicates mixing although the interfacial roughness approaches the bottom layer thickness of $438 \AA$.

The NFA and conjugated polymer samples were measured at INTER and D17 respectively. 


\subsection{Micron-sized crystal morphology}

In this section we are looking at the formation of PCBM crystals in PCBM/PS bilayers. Whereas in the preceding chapters the annealing times were carefully controlled to minimise PCBM crystallisation (both nano-crystal and micron-sized), the purpose of this chapter is to explicitly examine the morphology of micron-sized crystals. This is achieved by using longer annealing times at higher temperatures. The results are found by optical microscopy and AFM.
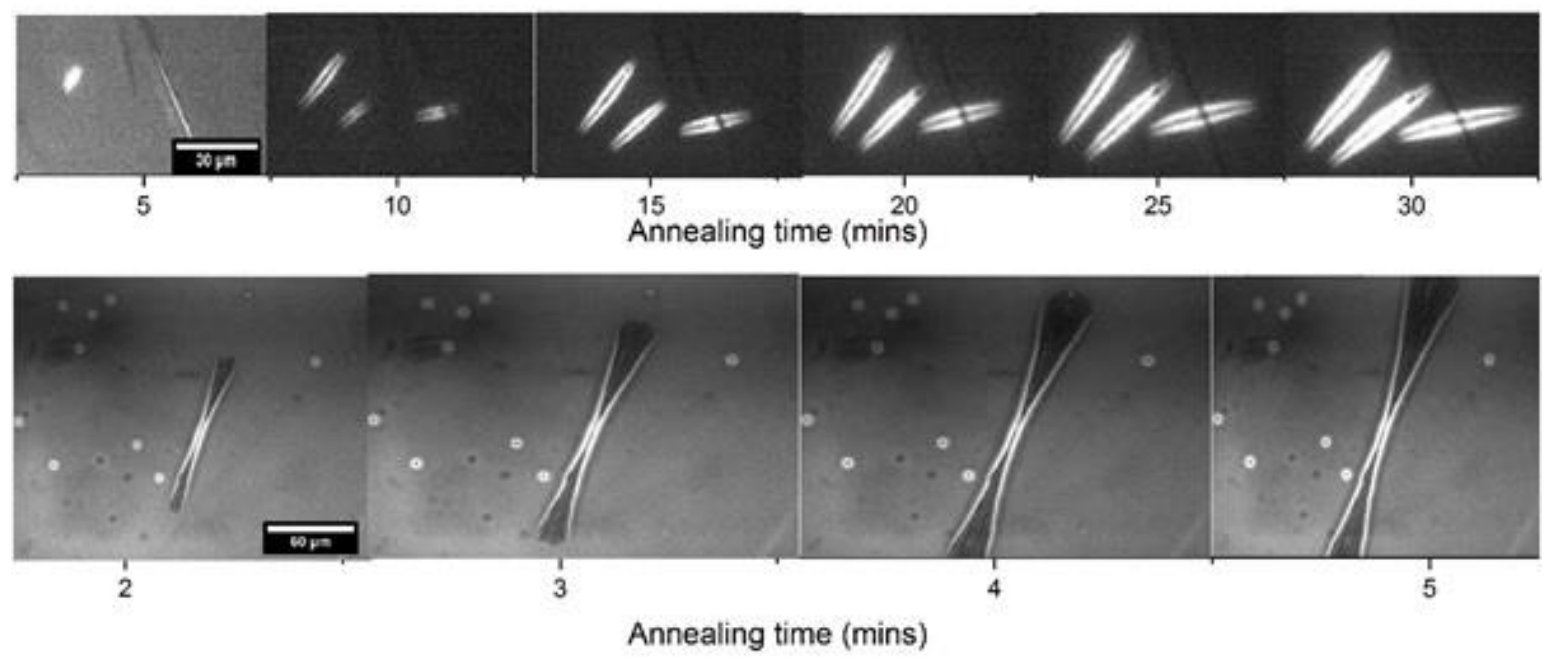

Figure 4.5-1: Growth of needles and fans for 25nm PCBM, PS 300k 40nm 180C (top) and PCBM 30nm, PS 20k 30nm 210C (bottom).

Figure 4.5-1 shows the growth of PCBM crystals in a bilayer following annealing. There were two types of crystal observed in this system which was seen to be dependent on the film thickness. This figure also highlights the effect of temperature on crystal growth rate as at higher temperatures we get much faster growth however with a lower nucleation density. The top morphology will be referred to as 'needle' and the bottom as 'branched' or 'fan'. In this section results from a study on this behaviour are shown with an emphasis on the fans as there has been significant work done on needle crystals by others (Môn et al. 2015). The primary interest here is not the growth-rate behaviour of the crystals (investigated extensively as a function of film thickness by Môn et al. 2015), but the nature of the morphology. In particular whether the crystals grow as needles or fans, and the branching behaviour within the fans. The crystal structure itself is not investigated in this study, but the needle-like crystals found in PCBM/PS bilayers (Môn et al. 2015) and PCBM/polymer blends 
(G. Li et al. 2008) are shown to be single crystals (using selected-area electron diffraction measurements). Both needles and branched crystallites have been observed in pure PCBM films annealed at 220Cand 240C respectively by Zheng et. al (Zheng et al. 2011).

\subsection{1 'Phase diagrams' for different layer thicknesses, $M W$, and temperature.}

To probe the effect of film thickness on the formation of crystals of differing morphology, a sequence of samples was prepared with a PCBM nominal thickness of $15,25,30,35$ and $55 \mathrm{~nm}$ and PS thicknesses of 20, 30, 40, 50 and 60nm. The thicknesses measured by AFM are shown in the appendix to this chapter. These samples used both $20 \mathrm{k}$ and $300 \mathrm{k}$ MW PS. Two temperatures were looked at: $170^{\circ} \mathrm{C}$ and $180^{\circ} \mathrm{C}$.

The samples were annealed in-situ on the optical microscope for different times, chosen to allow the crystals to form to a visible degree without impinging on the other crystals. Annealing took place in the dark, with the microscope shutter opened periodically to take images. It was observed that annealing time does not cause a transition between needles and fans but rather the morphology is decided from the initial nucleation with, in some cases, very small $(10-20 \mu \mathrm{m})$ fans appearing with the distinctive branching of the crystal front. Typical optical micrographs of PCBM crystals for different layer thicknesses are shown in Figure 4.5-2 to 4.5-5, with corresponding morphological categorisations ('phase diagrams') given in Figure 4.5-9. Examination of these figures reveals that the PS thickness does not have a major effect on whether the crystal morphology is needle-like or fan-like, nor does a $10{ }^{\circ} \mathrm{C}$ change in the temperature or the PS MW. There are some differences between the morphological categorisations with temperature and PS layer thickness, but the clearest finding is the overall transition from needle-like crystals to fan-like crystals as the PCBM thickness is increased, with the appearance of fans for PCBM thicknesses of around 30nm or above. Other studies have found the occurrence of both needles and fans in PCBM/polymer blends but have reported the transition between these two morphologies to be controlled by annealing temperature (Wong et al. 2014) or the ratio of the blend (Swinnen et al. 2006). The ratio of the layer 
thickness in this study was not found to have a discernible effect but rather the only acting parameter was the PCBM thickness.

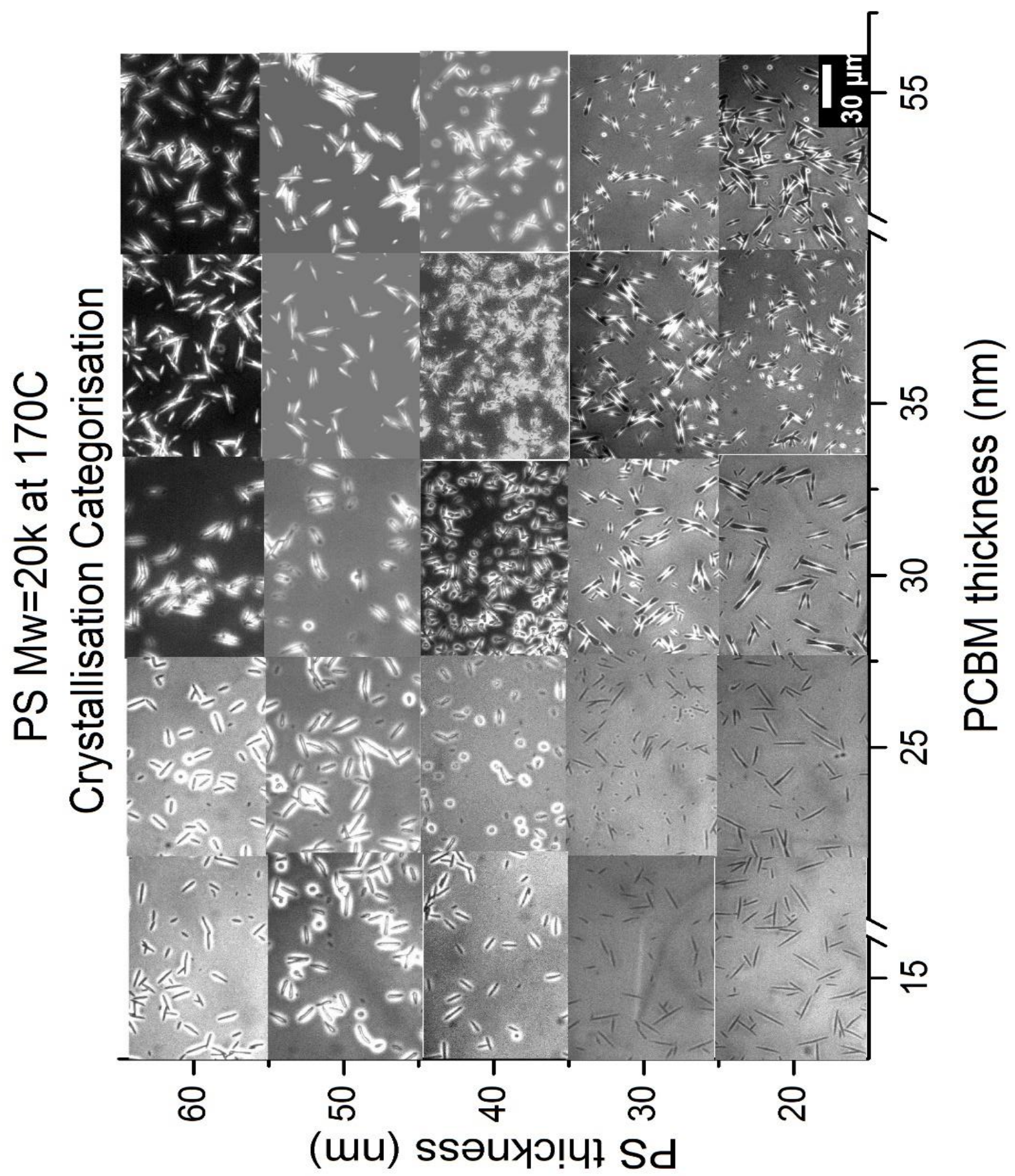

Figure 4.5-2: OM images of samples annealed at 170C for PCBM/PS bilayers with $M W=20 k$ with the thickness of each layer indicated by the axes. 


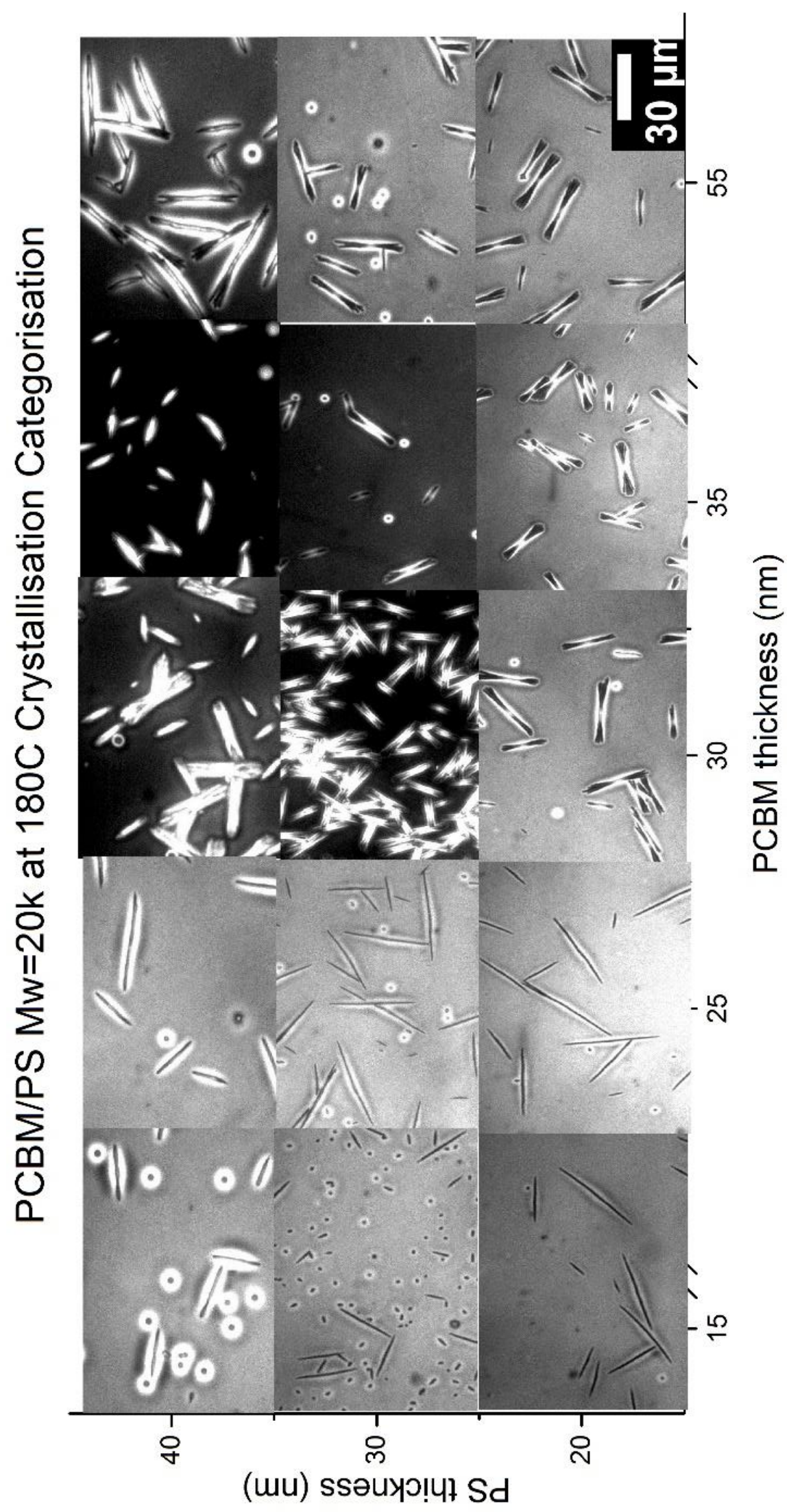

Figure 4.5-3: OM images of samples annealed at 180C for PCBM/PS bilayers with $M W=20 k$ with the thickness of each layer indicated by the axes. 


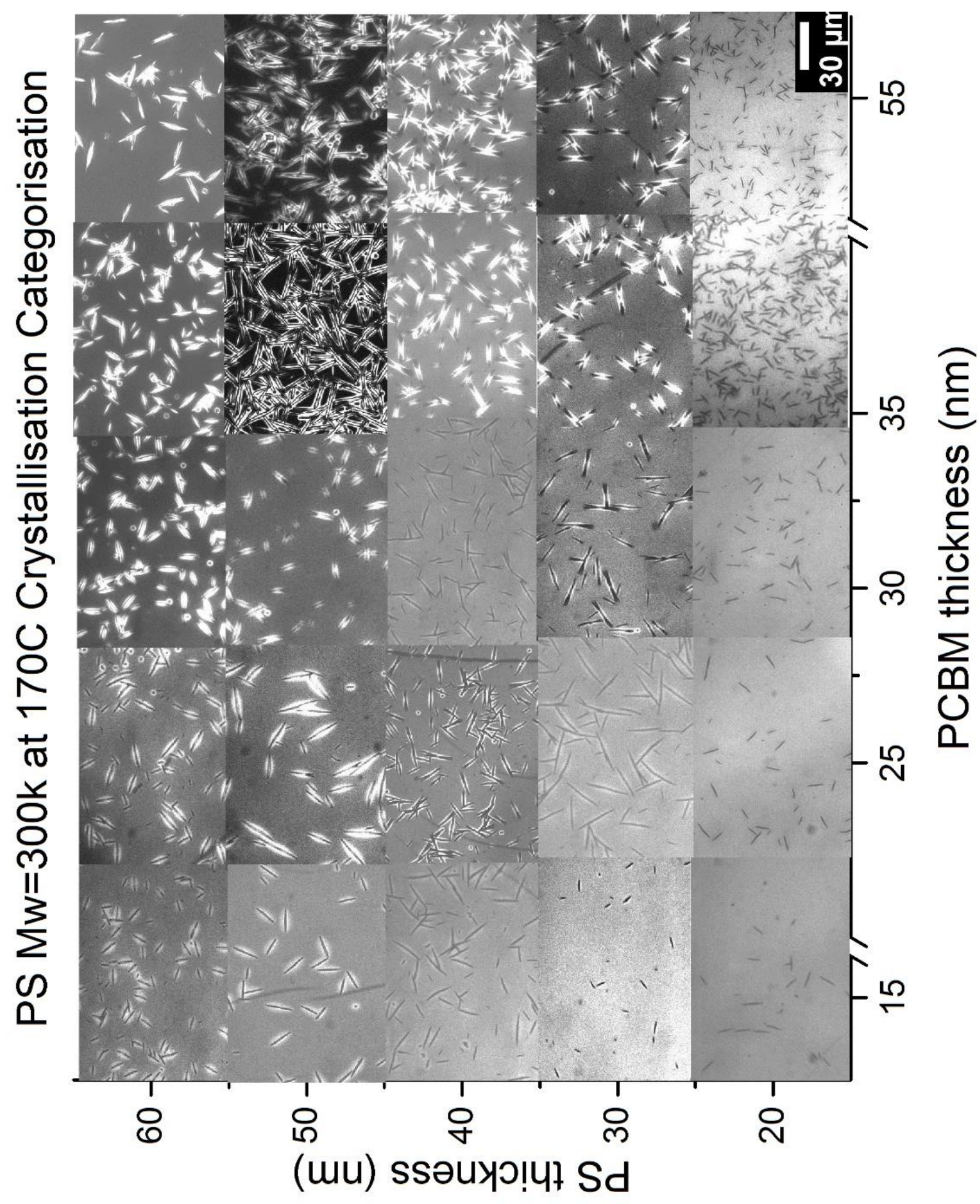

Figure 4.5-4: OM images of samples annealed at 170C for PCBM/PS bilayers with $M W=300 k$ with the thickness of each layer indicated by the axes. 


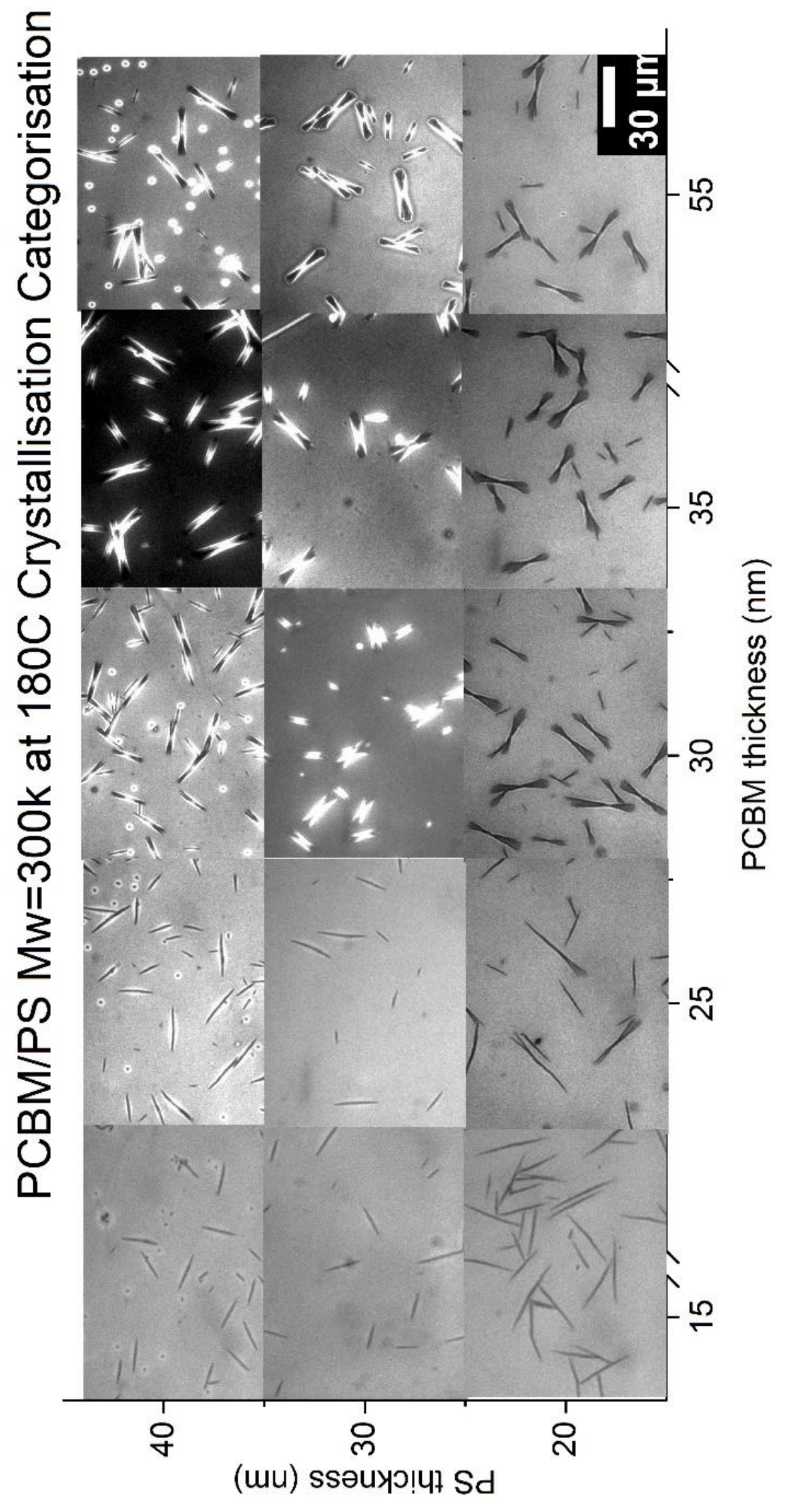

Figure 4.5-5:: OM images of samples annealed at 180C for PCBM/PS bilayers with $M W=300 k$ with the thickness of each layer indicated by the axes. 


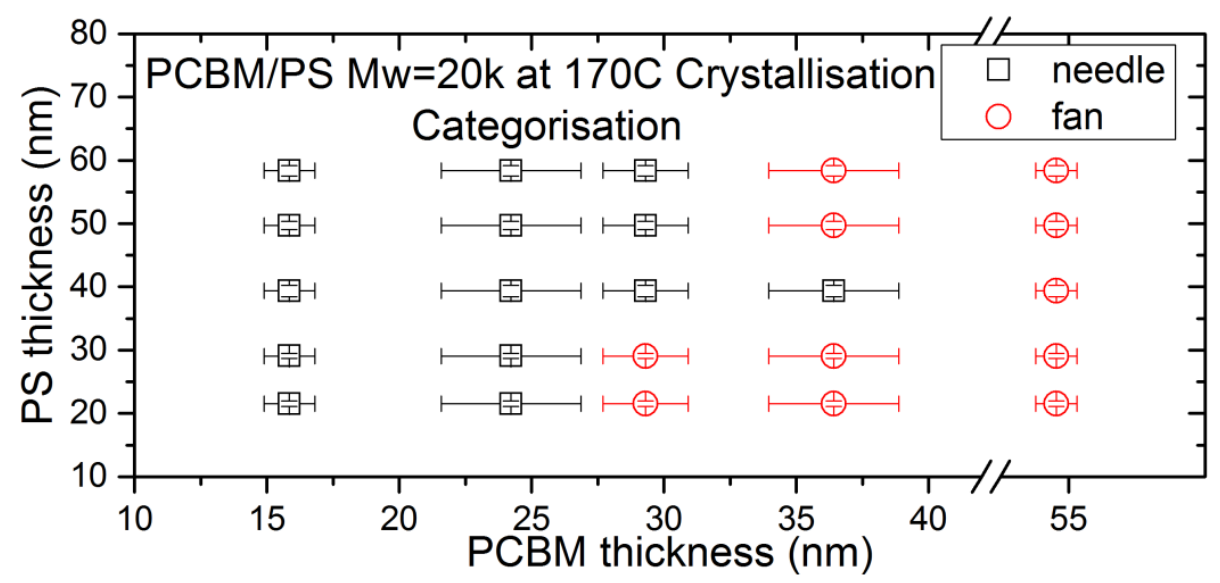

Figure 4.5-6: Categorisation of observed morphologies for the preceding OM images.

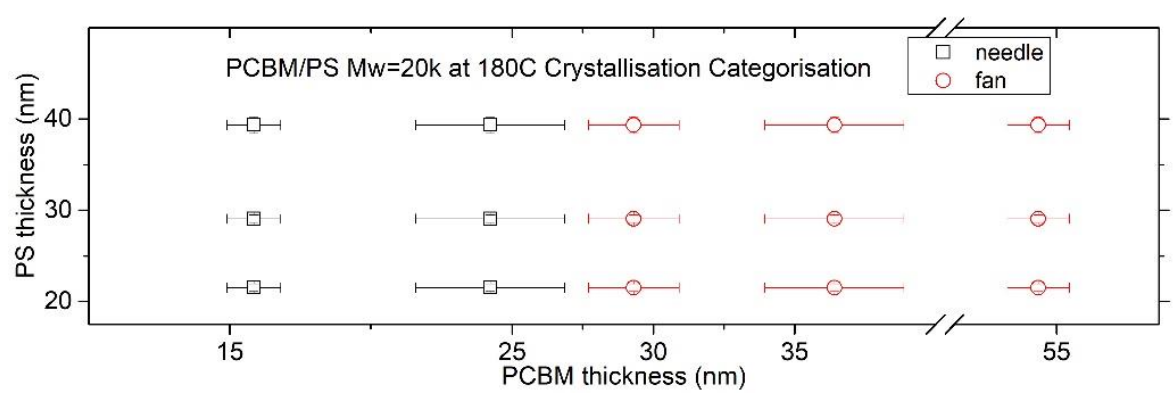

Figure 4.5-7: Categorisation of observed morphologies for the preceding OM images.

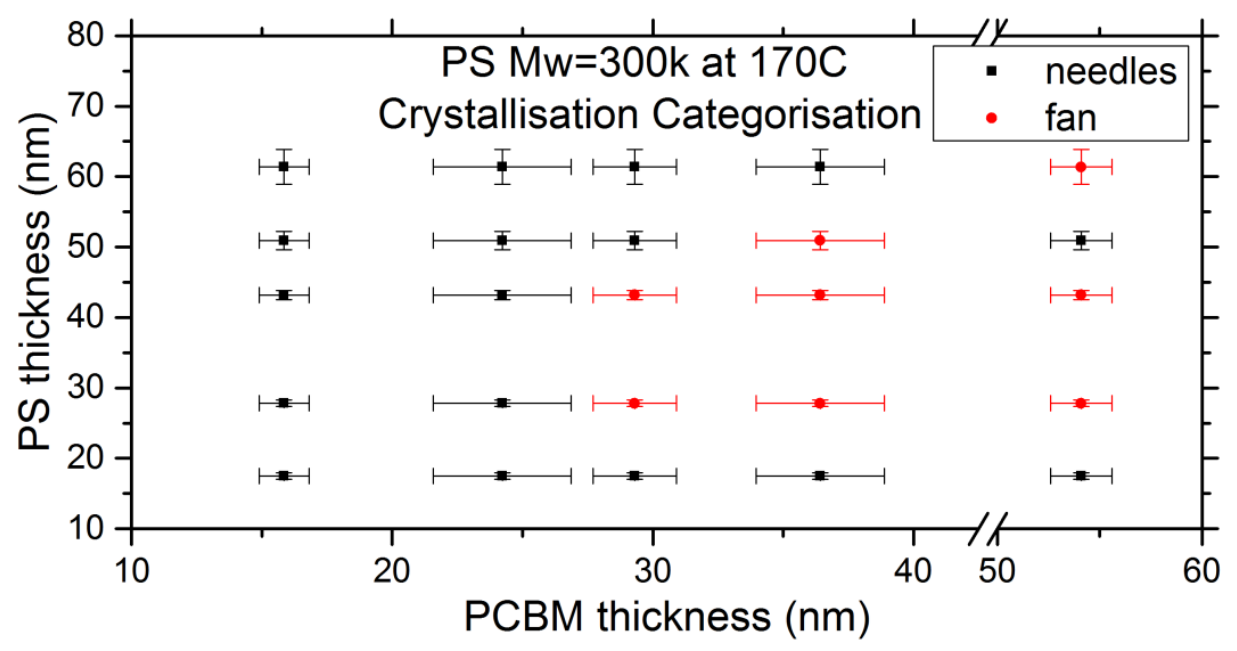

Figure 4.5-8: Categorisation of observed morphologies for the preceding OM images. 


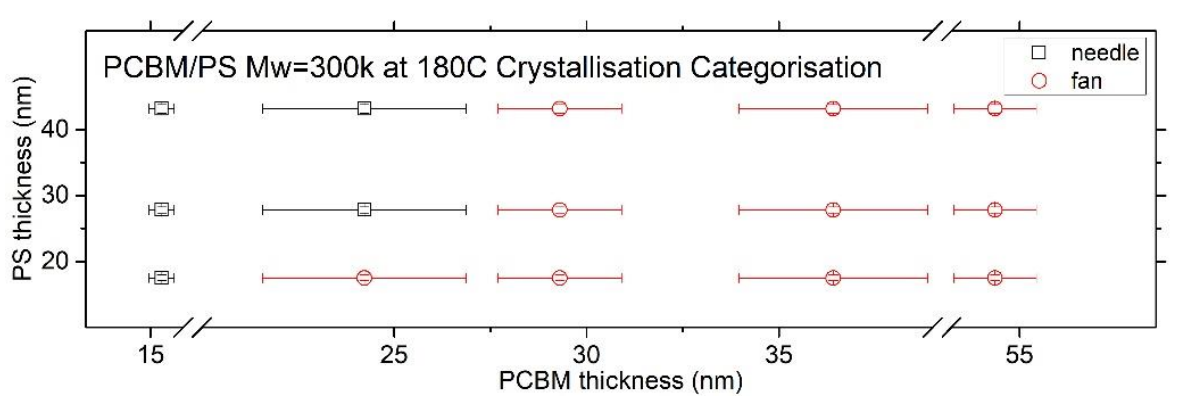

Figure 4.5-9: Categorisation of observed morphologies for the preceding OM images.

\subsubsection{Temperature dependence of fan morphology}

In this section the effect of temperature on the crystal morphology will be presented; in particular the morphology of the fan-like crystals. Figures 4.5-10 and 4.5-11 show a series of optical microscopy and AFM images from samples annealed at a range of different temperatures. The fans grow from an initial stem that resembles a needle in optical microscopy but when looked at in AFM it is clear there are ridges on the uppermost surface, as in Figure 4.5-12, that then split into separate branches or fibrils. Temperature clearly has a strong effect on the size and growth rate of these crystals. 

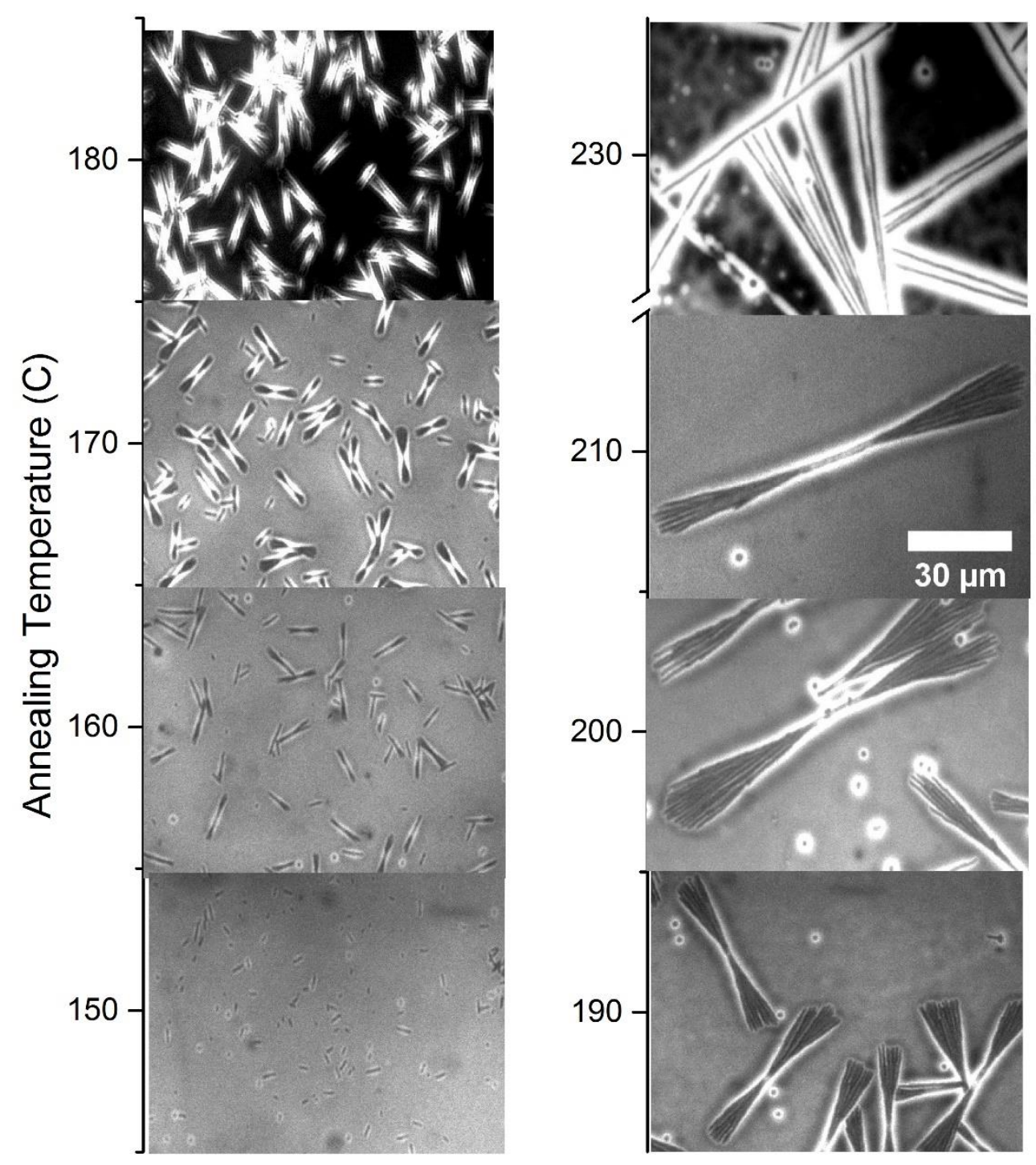

Figure 4.5-10: OM images of fans for samples annealed at different temperatures showing a decrease in nucleation density and increased dimensions with increasing temperature. Data are shown for the PCBM and PS thicknesses that are both 30nm with PS MW of $20 k$.

Figure 4.5-10 shows OM images of fan crystals showing the increase in size and decrease in nucleation density of the crystals as the temperature is increased. The focus of this chapter is to qualitatively categorise crystal morphology as either a needle or a fan. Quantifying the characteristics of the crystals poses problems. 
Measurement of the width of fan crystals (trough to trough distance as shown in Figure 4.5-12) at the nucleation site (the 'stem width'; see Figure 4.5-13) reveals similar behaviour with temperature to that found by Mon et al for needles(Môn et al. 2015). The other parameter that could be measured is the width of the fibrils within the branching crystal microstructure, however the branching causes variations in the fibril width so this parameter is difficult to quantify.

Mostly the branching fibrils appear to fill the space between the outermost arms of the fan crystals (eg; see Figure 4.5-14, Figure 4.5-15 and Figure 4.5-16). Fibril branching has been the subject of a number of theoretical studies, with proposed branching mechanisms including tip-splitting instabilities (due for example to the behaviour of temperature or impurity gradients that occurs when growing planar crystal front are subject to a pertubation) (Langer 1980; Crist and Schultz 2016) and non-crystallographic branching (due to nucleation of new crystallographic orientations at the growing crystal front) (Granasy et al. 2014). However, the fibril width is not easy to quantify experimentally. There are a range of fibril widths within a particular crystal (there is a range of at least a factor of two, either side of the point where a single fibril branches into two fibrils, and some crystals show a much broader range of feature sizes). The branching also seems to be halted within some samples, where non-branching fibrils overtake branching fibrils after some initial period of time. This is shown in figure 4.5-15; branching fibrils closer to the nucleation site are overtaken by fibrils that do not branch (even though those near to the growing tip do not appear to be impeded by neighbouring fibrils). Careful inspection of figure 4.5-15 also shows some kind of 'frustrated bifurcation' in the non-branching fibrils, where an indentation runs along the centre of the fibrils. Finally, this non-branching behaviour is also associated with the depressions either side of the fibrils (regions from which the bottom PCBM layer has been depleted to form the growing crystal (Môn et al. 2015)) going right down to a constant depth (indicative of full depletion of the bottom PCBM layer, leaving only the PS layer). This is shown in Figure 4.5-19 where non-branching fibrils are all bounded by fully depleted depressions, in regions that are sufficiently far from the crystal nucleation site. We would expect from some theories (Gránásy et al. 2004) that the fans might be precursors to spherulites, however, we do not see much evidence of significant curvature in the crystal edges in any samples. Interestingly, the branching density is 
significantly lower in some of the higher temperature samples (eg; the $230^{\circ} \mathrm{C}$ sample in Figure 4.5-11, and the $190{ }^{\circ} \mathrm{C}$ sample in Figure 4.5-10). However, there is not sufficient data to state such temperature-dependent effects categorically.

Overall, varied crystal morphologies have been observed, with a systematic dependence of this on PCBM layer thicknesss. At present it is not clear exactly why this thickness dependence occurs, or what controls the value of the transition thickness between fans and needles (at around 30nm).

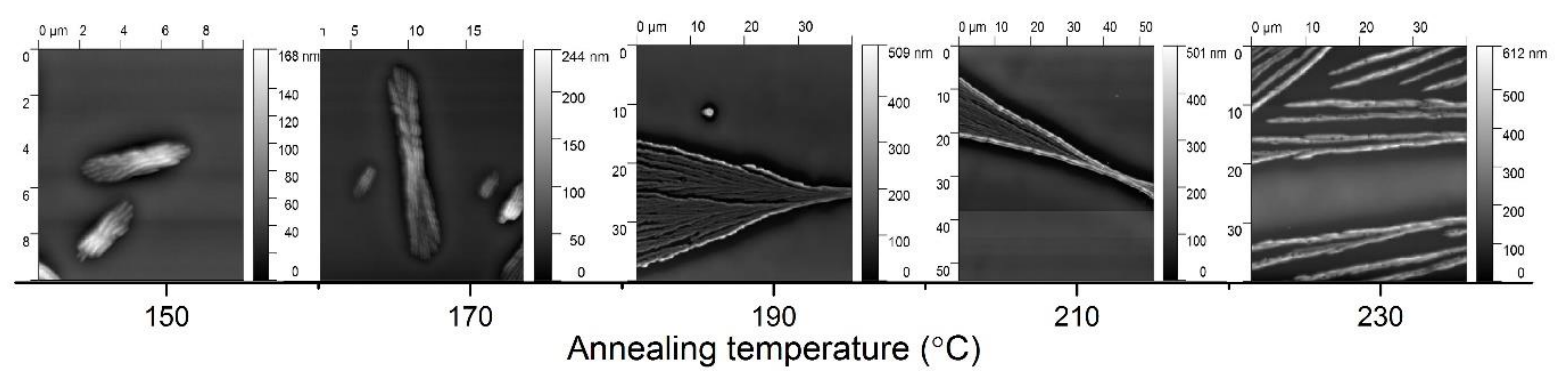

Figure 4.5-11: AFM images showing morphology development for fans annealed at different temperatures for different times (until crystal growth slowed or was impeded). The size of the scan is shown on the axis. PCBM and PS thickness es are both 30nm. The PS MW is $20 \mathrm{k}$.

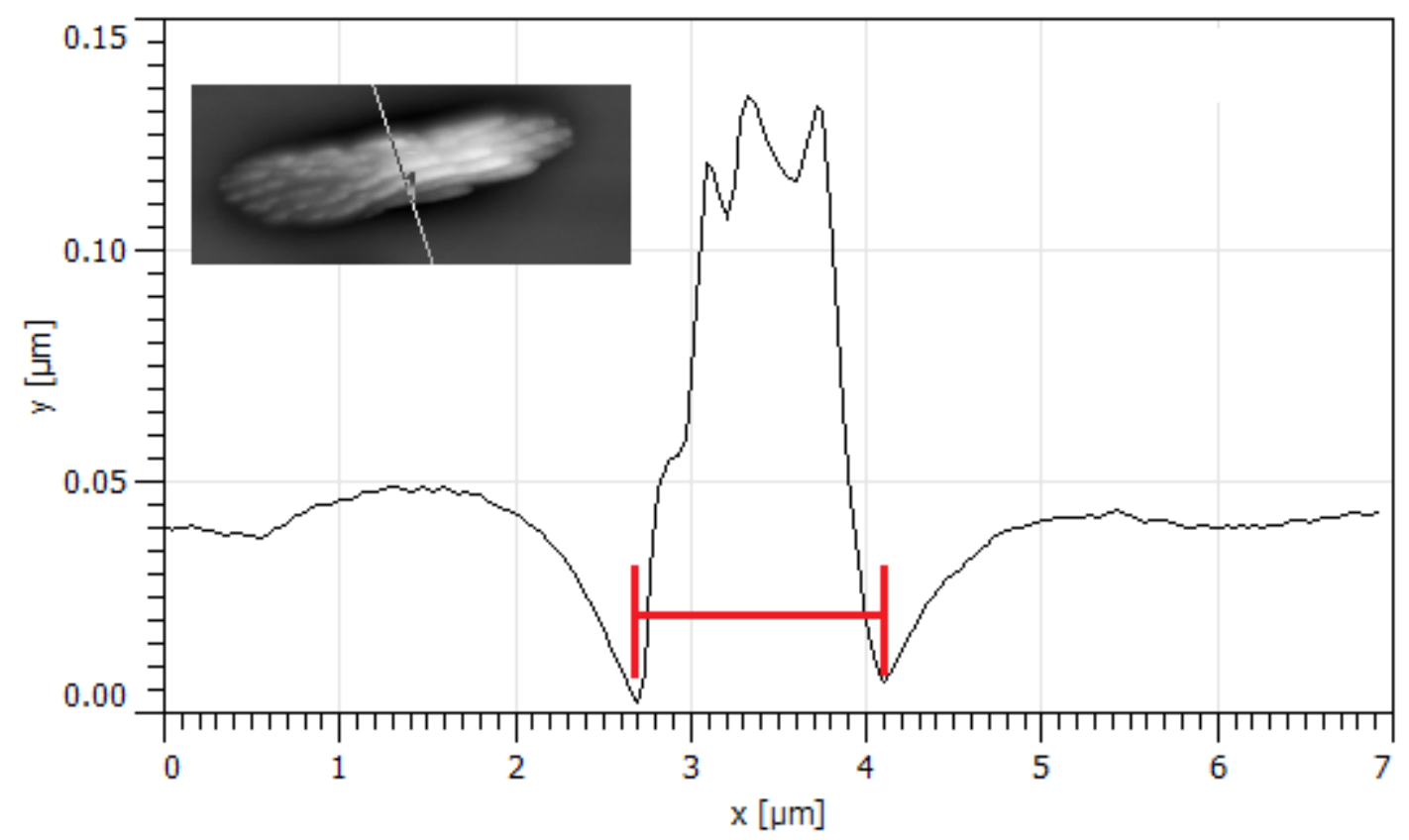

Figure 4.5-12: A profile of a fan taken normal to the growth direction indicating the depletion zones to either side of the crystal. The red line shows a stem width from trough to trough on either side of the crystal.PS 20k 30nm PCBM 30nm 150C. 


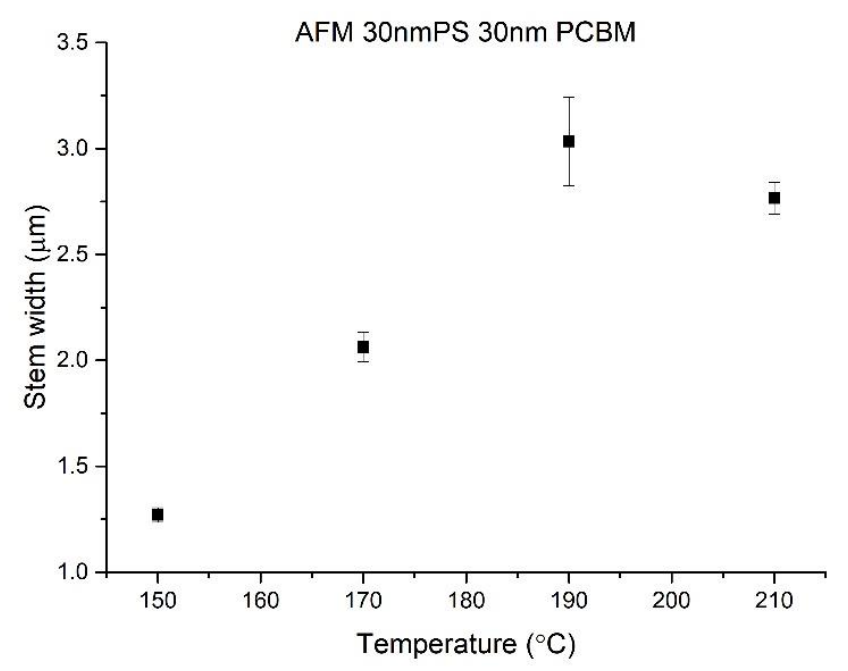

Figure 4.5-13: Stem width measured by AFM for different annealing temperatures. Values averaged over multiple crystals.

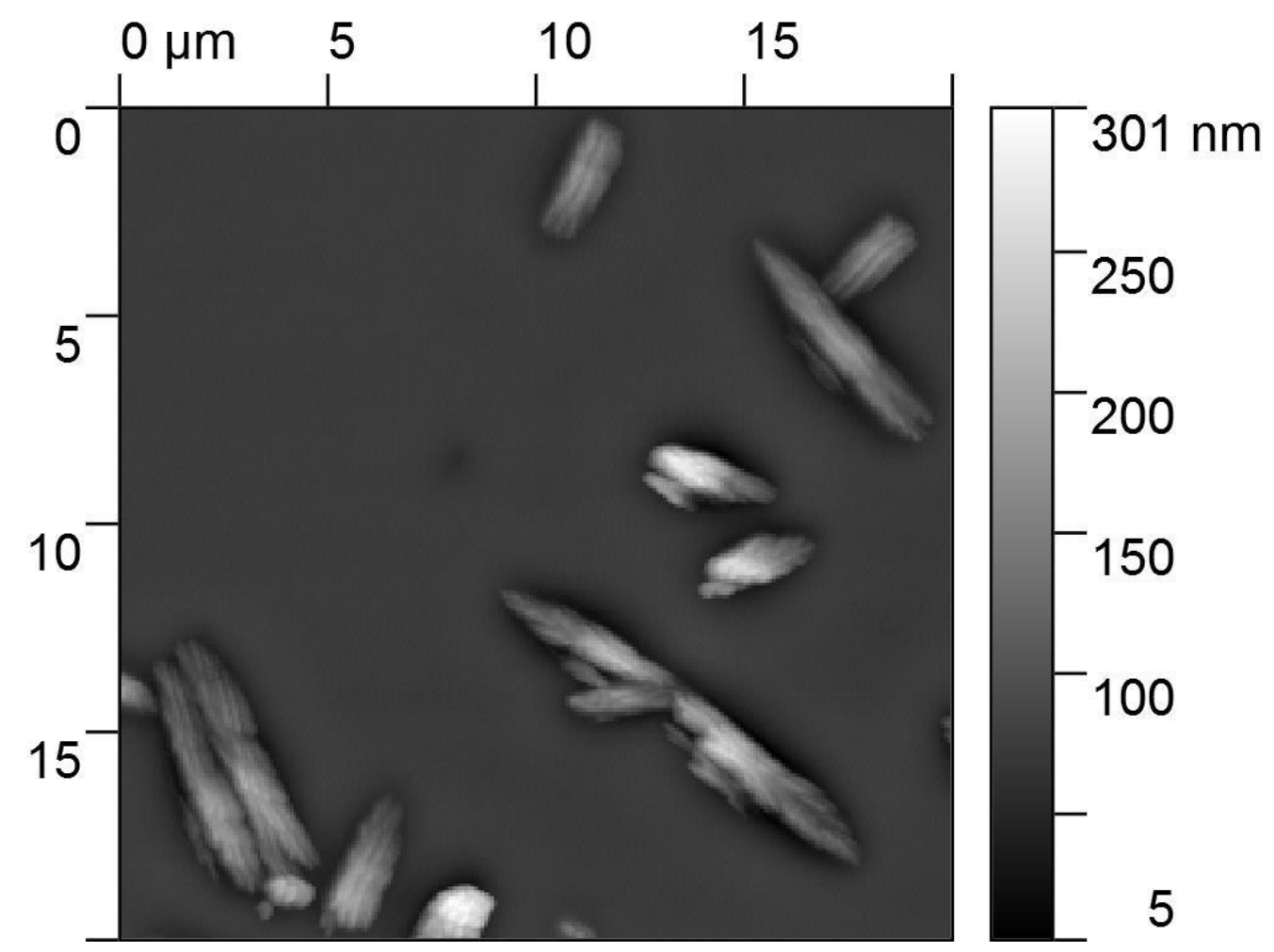

Figure 4.5-14: 30nm PS and 30nm PCBM film annealed at $150^{\circ} \mathrm{C}$ showing distinct branching of the crystals. 


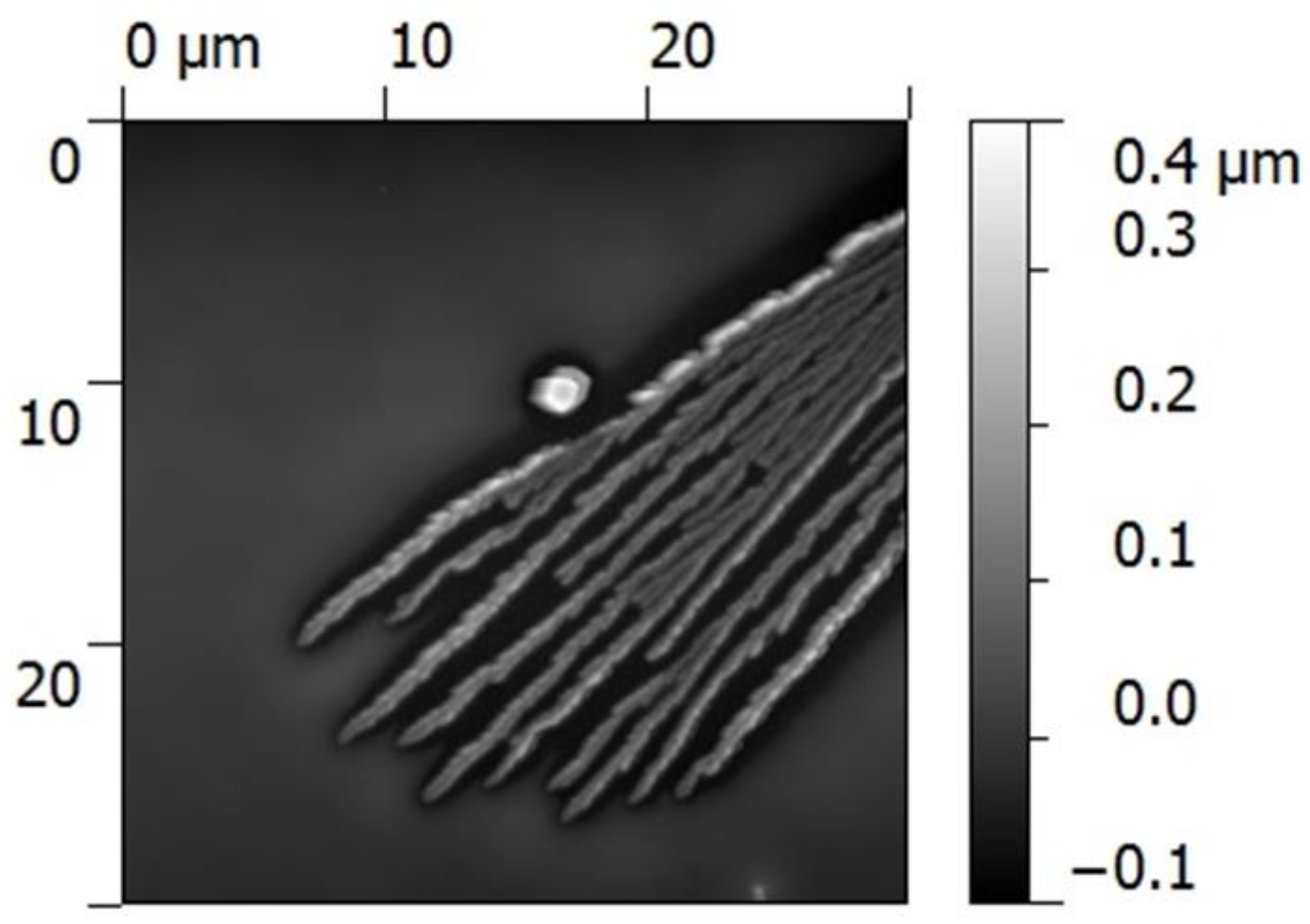

Figure 4.5-15: $30 \mathrm{~nm}$ 20k PS and 30nm PCBM film annealed at $190^{\circ} \mathrm{C}$ showing dinstinct branching of the fibrils within thicker outer arms.

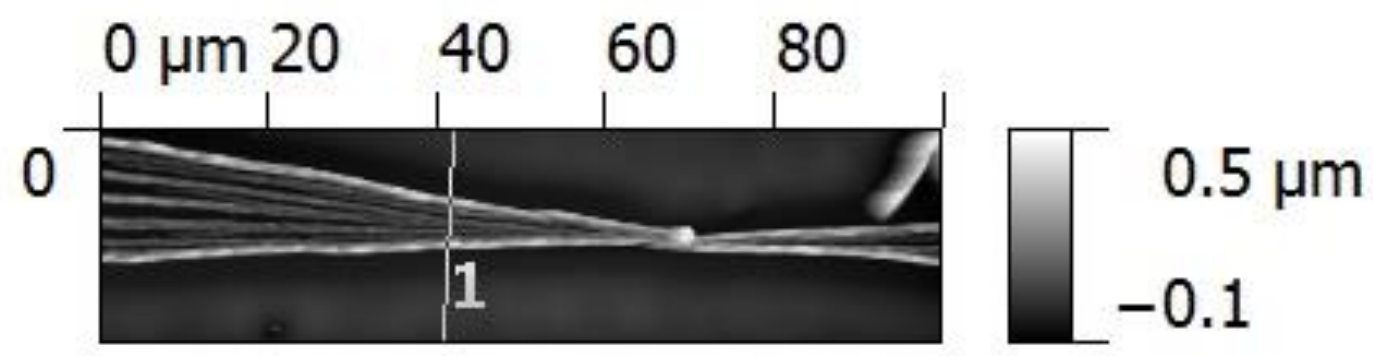

Figure 4.5-16: 30nm PS and 30nm PCBM film annealed at $230^{\circ} \mathrm{C}$ showing isolated branches with a broad width. 


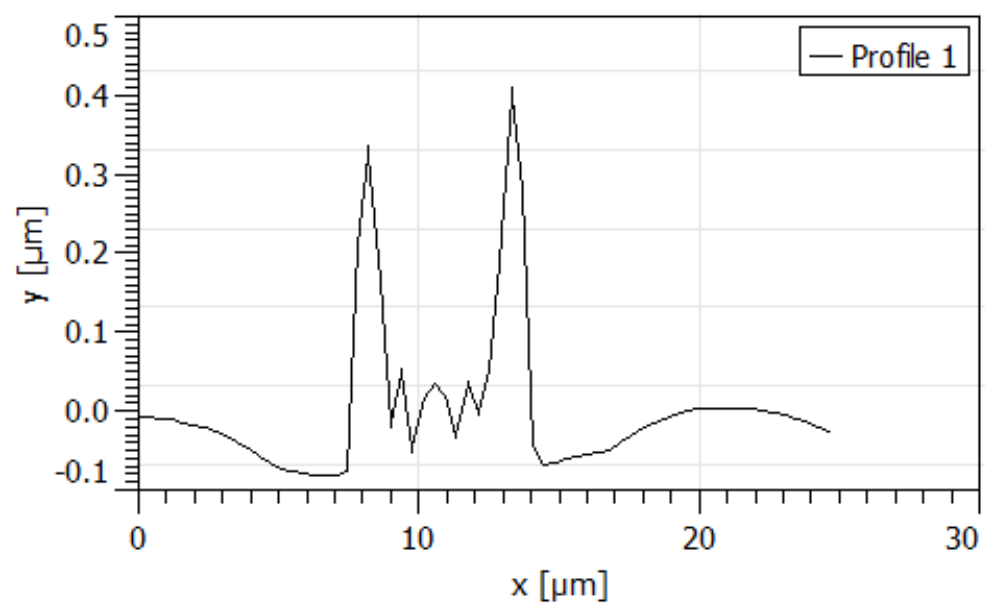

Figure 4.5-17: Surface profile for the line shown in figure 4.5-16

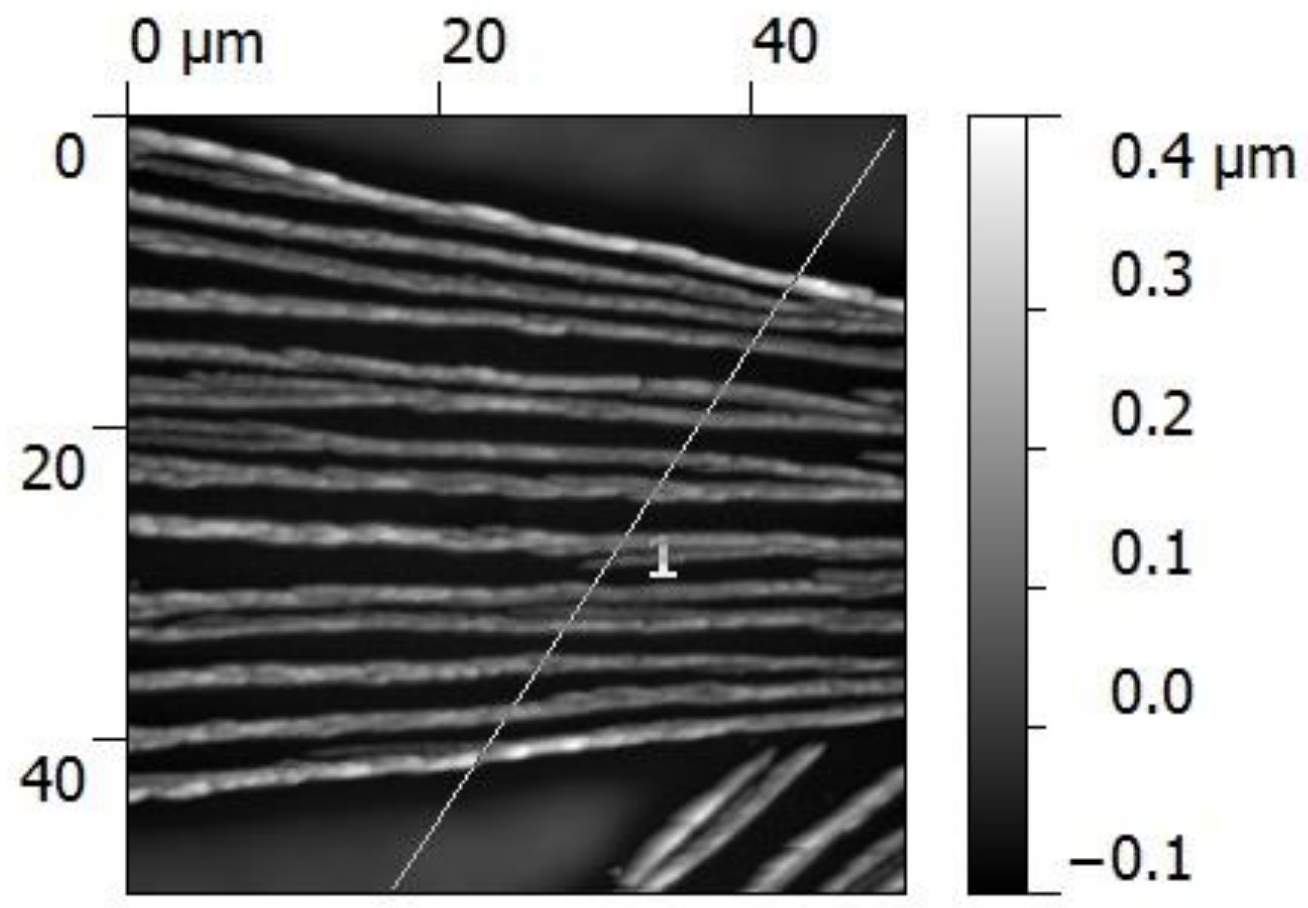

Figure 4.5-18: $30 \mathrm{~nm}$ PS and 30nm PCBM film annealed at $230^{\circ} \mathrm{C}$ showing isolated branches with a broad width. This is the same crystal that is shown in figure 4.5-15. 


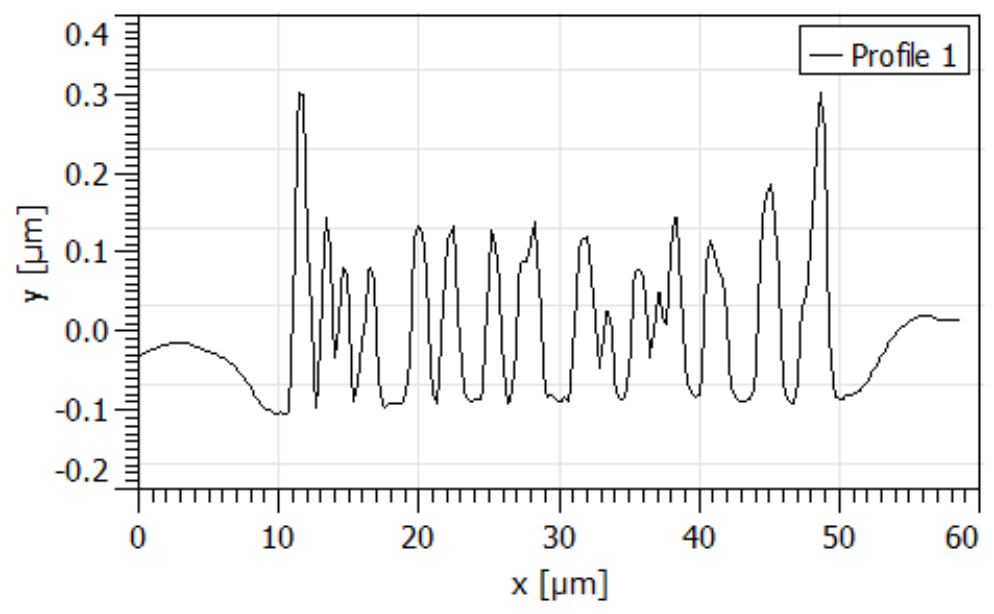

Figure 4.5-19: Surface profile for slice indicated in figure 4.5-18

\subsubsection{Crystal growth}

Crystal length for individual crystals is shown in Figure 4.5-20 and Figure 4.5-21 as a function of annealing time and temperature respectively. These results are in broad agreement with measurements made by Mon et. al, (who see an approximately constant growth rate on these timescales in films with PS thicknesses of around 40 $\mathrm{nm}$ or above). The annealing temperature is seen to have an effect on the rate of growth, with a higher growth-rate at higher temperature. For a more detailed analysis see Mon et al.

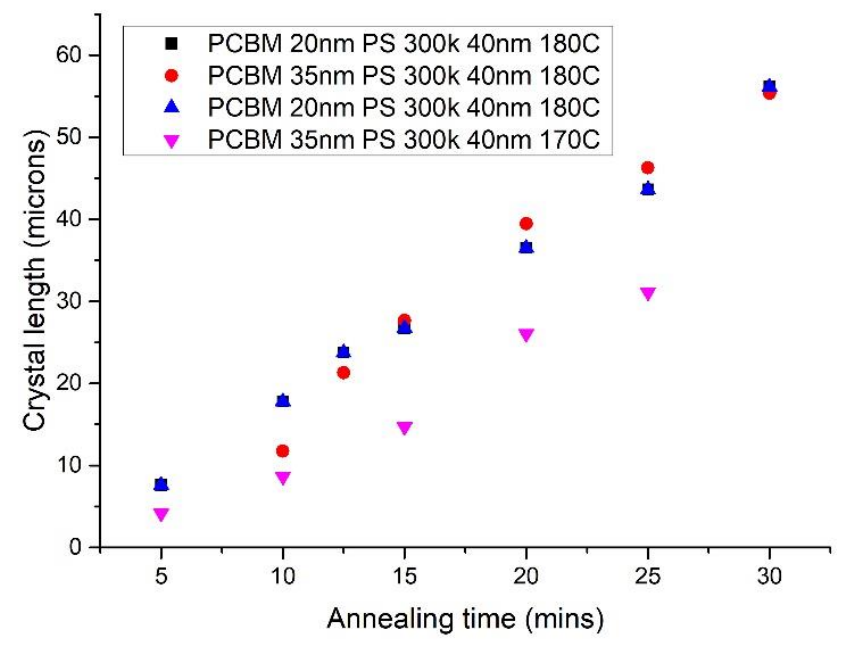

Figure 4.5-20: Crystal length measured on single crystals for progressive annealing times (measured by optical microscopy). Layer thicknesses and MW shown in legend. 


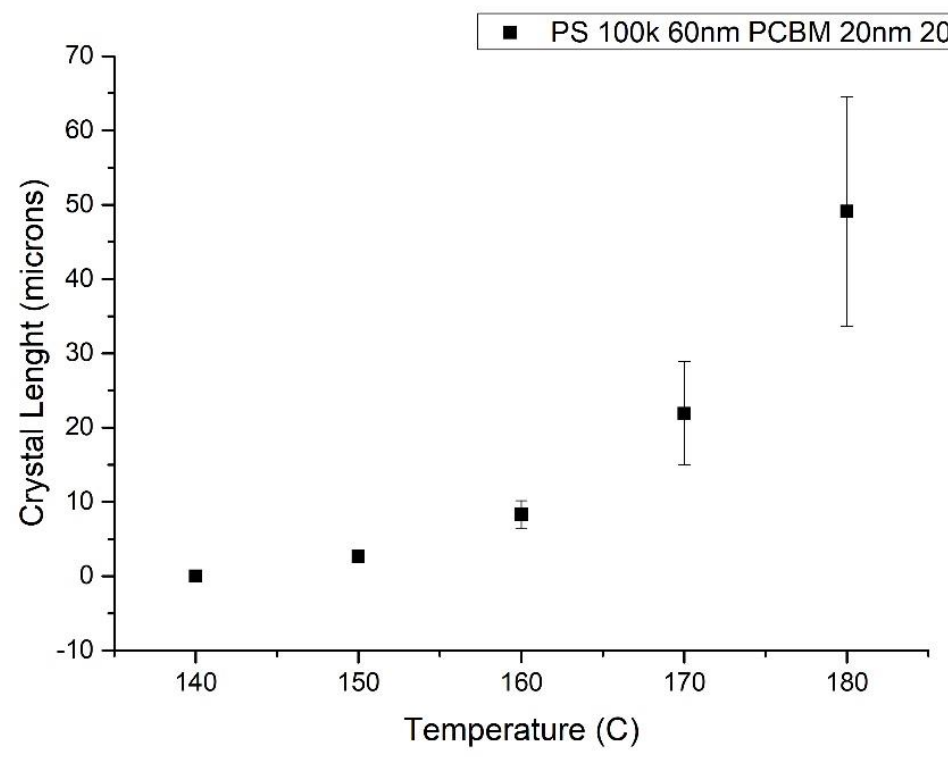

Figure 4.5-21: Crystal length (measured by optical microscopy) for unimpeded crystals at different temperatures for layer thicknesses and MW shown in legend.

\subsubsection{Appendix}

\begin{tabular}{|c|c|c|c|}
\hline $\begin{array}{l}\text { Spinning speed } \\
\text { (krpm) and concen- } \\
\text { tration }(\mathrm{m} / \mathrm{m}) \text { and } \\
\text { MW of PS }\end{array}$ & $\begin{array}{l}\text { Nominal thick- } \\
\text { ness }(\mathrm{nm})\end{array}$ & $\begin{array}{l}\text { Thickness meas- } \\
\text { ured by AFM (nm) }\end{array}$ & $\begin{array}{l}\text { Error of AFM meas- } \\
\text { urement (standard } \\
\text { deviation) }(\mathrm{nm})\end{array}$ \\
\hline $3 \mathrm{k}, 1 \%, 20 \mathrm{k}$ & 20 & 21.5 & 1.12 \\
\hline $2 \mathrm{k}, 1 \%, 20 \mathrm{k}$ & 30 & 29.1 & 1.06 \\
\hline $3 \mathrm{k}, 1.5 \%, 20 \mathrm{k}$ & 40 & 39.3 & 2.47 \\
\hline $3 \mathrm{k}, 2 \%, 20 \mathrm{k}$ & 50 & 49.7 & 1.71 \\
\hline $2 \mathrm{k}, 2 \%, 20 \mathrm{k}$ & 60 & 58.4 & 2.15 \\
\hline $2 \mathrm{k}, 0.5 \%, 300 \mathrm{k}$ & 20 & 17.5 & 1.15 \\
\hline $3 \mathrm{k}, 1 \%, 300 \mathrm{k}$ & 30 & 34 & 3.81 \\
\hline $1.5 \mathrm{k}, 1 \%, 300 \mathrm{k}$ & 40 & 42.1 & 1.3 \\
\hline $3 \mathrm{k}, 1.5 \%, 300 \mathrm{k}$ & 50 & 53.9 & 2.49 \\
\hline $2 \mathrm{k}, 1.5 \%, 300 \mathrm{k}$ & 60 & 61.4 & 6.18 \\
\hline \multicolumn{4}{|l|}{$\begin{array}{l}\text { Spinning speed } \\
(\mathrm{krpm}) \text { and concen- } \\
\text { tration }(\mathrm{m} / \mathrm{m}) \text { of } \\
\text { PCBM }\end{array}$} \\
\hline $3 \mathrm{k}, 1 \%$ & 15 & 15.9 & 2.54 \\
\hline $3 \mathrm{k}, 1.5 \%$ & 25 & 21.6 & 6.29 \\
\hline $1.5 \mathrm{k}, 1.5 \%$ & 30 & 29.3 & 4.24 \\
\hline $1.5 \mathrm{k}, 2 \%$ & 35 & 36.4 & 6.51 \\
\hline $3 \mathrm{k}, 3 \%$ & 55 & 54.1 & 3.99 \\
\hline
\end{tabular}




\section{Conclusions}

This thesis focused on the composition of polymer/fullerene bilayers following annealing as a function of MW, annealing temperature and layer thicknesses. Coexisting compositions, interfacial roughness, and crystal morphology were examined.

The system studied in greatest detail was PCBM/PS. Here, the interfacial roughness measured by NR was found to increase for lower MW. Coexisting compositions of $10 \%$ and $100 \%$ PCBM were formed following annealing above the glass transition. There was no temperature dependence or MW dependence observed in the range looked at $\left(120^{\circ} \mathrm{C}-180^{\circ} \mathrm{C}\right.$ and $\left.2 \mathrm{k}-300 \mathrm{k}\right)$. The observed equilibrium between these two phases (and the stable interfacial roughness) was formed in less than five minutes as determined using in-situ measurements. There was no MW dependence of the coexisting compositions in this system. Flory-Huggins theory was found to agree with experiment, within error, for a chi parameter value of 2 (referenced to a lattice size of a PCBM molecule). This value of chi predicts a slight variation in composition for low MW (2k), but the NR measurements were not sensitive to such a small change. The MW dependence of the interfacial roughness was seen to be in qualitative agreement with SCFT theory for a polymer/polymer interface although the values predicted were a factor of around 3 smaller than observations. None of the findings contradicted the hypothesis that this system formed a liquid-liquid equilibrium, within the range of annealing temperatures and times used.

Bis-PCBM/PS bilayers were also tested to see what effect the extra side chain had in comparison to the PCBM/PS system. This fullerene is also less prone to crystallise, making it more ideal for measurements below the melting point which could lead to crystallisation in the PCBM system (at longer annealing times). In the bis-PCBM/PS system, we found similar behaviour to the PCBM/PS: broader interfaces for lower MW and coexisting compositions retaining a bilayer geometry after annealing. However, in this system more dramatic changes were observed as a function of MW and annealing temperature. To such an extent that the interfacial roughness observed for the lower MW of these systems approached the thickness of the bottom layer, 
rendering comparisons with error function (or tanh) profiles from SCFT predictions potentially inappropriate. The Flory-Huggins chi parameter value obtained from the higher PS MW (20-100k) was 1.5 (referenced to a lattice size of a bis-PCBM molecule). There was extensive mixing in both the top and bottom layers for lower MW and this behaviour, compared to PCBM/PS, is what would be expected. This is because the extra side-chain makes the bis-PCBM more chemically similar to the PS molecules than are the PCBM molecules, which encourages mixing. The temperature range used for this system was $140-145^{\circ} \mathrm{C}$ with one sample at $180^{\circ} \mathrm{C}$ showing a broader interface despite being of a higher MW (100k). This suggests some temperature dependent behaviour.

The effects of different storage conditions of the PCBM/PS system prior to annealing were also explored. This was done by observing the effect of storage under various conditions (dark, light, air and inert atmospheres) on bilayers left for 1-4 weeks. Exposure to light is known to affect PCBM in two ways: oxidation if simultaneously exposed to air, and oligomerisation if illuminated in an inert atmosphere. It was found the exposure to light and air, and extensive oligomerisation ( 40\%) altered the observed depth profiles measured by NR when compared to the non-illuminated samples. This was seen by a depressed SLD of the bottom layer for the samples exposed to light and air (for lower MW only) and a broader interface observed in the $\sim 40 \%$ oligomerised sample. These results indicate that storage conditions for bilayers and materials, prior to device fabrication (including annealing), could affect the miscibility of the components. The results may also have implications for the long-term behaviour of devices in operation.

PCDTBT/PCBM and dPS/NFA bilayers were also looked at briefly. These were prepared as bilayers, annealed above their glass transition temperatures and measured with NR. These combinations were found to be highly miscible with a single layer emerging as the best fit for the PCDTBT/PCBM samples and a bilayer with two very close SLD values for the NFA/dPS sample.

\subsection{Future Work}

There is a stronger dependence on MW and temperature in the bis-PCBM systems, and in future it is recommended to use thicker bis-PCBM layers, as significant diffusion and interfacial roughness formation is seen, that requires thicker layers to fully characterise. 
The in-situ results suggest temperature dependence of mixing in the PCBM/PS systems. However quite large temperature steps of $10-20^{\circ} \mathrm{C}$ were used, making it difficult to discern the effects of temperature. Future work could examine this more closely by performing further in-situ experiments with smaller temperature steps. In terms of aging samples, there is some effect on samples aged in light and air, and also (tentatively) those that were extensively oligomerised which also would require further work to fully understand. The mixing has clearly been affected although this is not clearly associated with a lower chi-value, as this would be expected to affect the top layer composition also. It could be the case that there is a temperature, MW, or composition dependent chi at play in this system. Further work could include examination of samples aged in light and air, as a function of MW in more detail. The crystal morphology work has shown that there is a clear PCBM layer thickness dependence. Future work could probe the geometry of the crystals in terms of the branching angles and dimensions of the crystals formed using a wider range of MW and annealing temperatures.

In terms of the main contribution of the work in this thesis with-respect-to nanocomposite systems in general and OPV materials in particular, the key significance is that the findings in these potentially complex systems can be successfully interpreted in terms of equilibrium theories. It is hoped that this work can contribute directly to understanding aspects of OPV structure-development during fabrication and operation. In particular, it is hoped that such understanding will complement materials development and device optimisation work, and contribute to the continued efforts in the OPV community of enabling rational design, based on a thorough understanding of phase composition and interfacial properties within materials relevant to OPVs. 


\section{References}

Adhikari, Narayan P. 2012. "Interfacial Tension and Width of an Asymmetric Polymer Mixture.” Macromolecular Symposia 315 (1): 15-23. doi:10.1002/masy.201250502.

Ballantyne, Amy M., Lichun Chen, Justin Dane, Thomas Hammant, Felix M. Braun, Martin Heeney, Warren Duffy, Iain McCulloch, Donal D C Bradley, and Jenny Nelson. 2008. "The Effect of Poly(3-Hexylthiophene) Molecular Weight on Charge Transport and the Performance of Polymer: Fullerene Solar Cells." Advanced Functional Materials 18 (16): 2373-80. doi:10.1002/adfm.200800145.

Baran, Derya, Raja Shahid Ashraf, David A Hanifi, Maged Abdelsamie, Nicola Gasparini, Jason A Röhr, Sarah Holliday, et al. 2016. "Reducing the EfficiencyStability-Cost Gap of Organic Photovoltaics with Highly Efficient and Stable Small Molecule Acceptor Ternary Solar Cells.” Nature Materials 84 (November): 1-18. doi:10.1038/nmat4797.

Bates, Frank S., Jeffrey H. Rosedale, Petr Stepanek, Timothy P. Lodge, Pierre Wiltzius, Glenn H. Fredrickson, and Rex P. Hjelm. 1990. "Static and Dynamic Crossover in a Critical Polymer Mixture.” Physical Review Letters 65 (15): 1893-96. doi:10.1103/PhysRevLett.65.1893.

Bernardo, Gabriel, Nabankur Deb, Stephen M. King, and David G. Bucknall. 2016. "Phase Behavior of Blends of PCBM with Amorphous Polymers with Different Aromaticity." Journal of Polymer Science, Part B: Polymer Physics, 994-1001. doi:10.1002/polb.24002.

Binder, K. 1984. "Nucleation Barriers, Spinodals, and the Ginzburg Criterion." Physical Review 29 (1): 341-49. doi:10.1007/s13398-014-0173-7.2.

Binnig, G., and C. F. Quate. 1986. "Atomic Force Microscope.” Physical Review Letters 56 (9): 930-33. doi:10.1103/PhysRevLett.56.930.

Brabec, C. 2010. "Polymer-Fullerene Bulk-Heterojunction Solar Cells." Advanced Materials 23 (34) 18

Broseta, D, G H Fredrickson, E Helfand, and L Leibler. 1990. "Molecular-Weight and Polydispersity Effects At Polymer Polymer Interfaces.” Macromolecules 23 (1): 132-39.

Broseta, Daniel, Glenn H Fredrickson, Eugene Helfand, and Ludwik Leibler. 1990. "Molecular Weight and Polydispersity Effects at Polymer-Polymer Interfaces." Macromolecules 23 (1): 132-39. doi:10.1021/ma00203a023.

Campoy-Quiles, Mariano, Yoshihiro Kanai, Ahmed El-Basaty, Heisuke Sakai, and Hideyuki Murata. 2009. "Ternary Mixing: A Simple Method to Tailor the Morphology of Organic Solar Cells." Organic Electronics: Physics, Materials, Applications 10 (6). Elsevier B.V.: 1120-32. doi:10.1016/j.orgel.2009.05.028.

Carrillo, Jan-Michael Y, Rajeev Kumar, Monojoy Goswami, Bobby G Sumpter, and W Michael Brown. 2013. "New Insights into the Dynamics and Morphology of P3HT:PCBM Active Layers in Bulk Heterojunctions." Physical Chemistry Chemical Physics : PCCP 15: 17873-82. doi:10.1039/c3cp53271b.

Chen, Dian, Feng Liu, Cheng Wang, Atsuhiro Nakahara, and Thomas P Russell. 
2011. "Bulk Heterojunction Photovoltaic Active Layers via Bilayer." Nano Letters, 2071-78.

Chen, Huipeng, Raghavendra Hegde, J. Browning, and M. D. Dadmun. 2012. "The Miscibility and Depth Profile of PCBM in P3HT: Thermodynamic Information to Improve Organic Photovoltaics." Physical Chemistry Chemical Physics 14: 5635. doi:10.1039/c2cp40466d.

Clulow, Andrew J., Ardalan Armin, Kwan H. Lee, Ajay K. Pandey, Chen Tao, Marappan Velusamy, Michael James, et al. 2014. "Determination of Fullerene Scattering Length Density: A Critical Parameter for Understanding the Fullerene Distribution in Bulk Heterojunction Organic Photovoltaic Devices." Langmuir 30 (5): 1410-15. doi:10.1021/la403951j.

Crist, Buckley, and Jerold M. Schultz. 2016. "Polymer Spherulites: A Critical Review." Progress in Polymer Science 56. Elsevier Ltd: 1-63. doi:10.1016/j.progpolymsci.2015.11.006.

Dang, Minh Trung, Lionel Hirsch, and Guillaume Wantz. 2011. "P3HT:PCBM, Best Seller in Polymer Photovoltaic Research." Advanced Materials 23: 3597-3602. doi:10.1002/adma.201100792.

Ediger, M. D., and J. a. Forrest. 2014. "Dynamics near Free Surfaces and the Glass Transition in Thin Polymer Films: A View to the Future." Macromolecules 47: 471-78. doi:10.1021/ma4017696.

Eftaiha, Ala'a F, Jon-Paul Sun, Ian G Hill, and Gregory C Welch. 2014. "Recent Advances of Non-Fullerene, Small Molecular Acceptors for Solution Processed Bulk Heterojunction Solar Cells." Journal of Materials Chemistry A 2: 120113. doi:10.1039/C3TA14236A.

Ender, Sabine, Kai Lagenbach, Philipp Schrader, and Tim Zeiner. 2012. "Phase Diagram for Systems Containing Hyper-Branched Polymers." Polymers 4(1): 72-115.

Ferguson, Andrew J., Jeffrey L. Blackburn, and Nikos Kopidakis. 2013. "Fullerenes and Carbon Nanotubes as Acceptor Materials in Organic Photovoltaics." Materials Letters 90. Elsevier: 115-25. doi:10.1016/j.matlet.2012.08.145.

Forrest, J a, K DalnokiVeress, J R Stevens, and J R Dutcher. 1996. "Effect of Free Surfaces on the Glass Transition Temperature of Thin Polymer Films." Physical Review Letters 77 (111): 2002-5. doi:10.1103/PhysRevLett.77.2002.

Forrest, James A., and Kari Dalnoki-Veress. 2001. "The Glass Transition in Thin Polymer Films." Advances in Colloid and Interface Science 94 (1): 167-95. doi:10.1016/S0001-8686(01)00060-4.

Galagan, Yulia, and Ronn Andriessen. (March 16th 2012). Organic Photovoltaics: Technologies and Manufacturing, Third Generation Photovoltaics, Vasilis Fthenakis, IntechOpen, DOI: 10.5772/25901. Available from: https://www.intechopen.com/books/third-generation-photovoltaics/organicphotovoltaics-technologies-and-manufacturing

Gao, Siyang, Yung P. Koh, and Sindee L. Simon. 2013. "Calorimetric Glass Transition of Single Polystyrene Ultrathin Films." Macromolecules 46 (2): 56270. doi:10.1021/ma3020036.

Gisaxs.com. 2015. "Neutron Scattering Lengths." http://gisaxs.com/index.php/Neutron_scattering_lengths.

Gránásy, László, Tamás Pusztai, Tamás Börzsönyi, James a Warren, and Jack F Douglas. 2004. "A General Mechanism of Polycrystalline Growth.” Nature 
Materials 3 (September): 645-50. doi:10.1038/nmat1190.

Granasy, Laszlo, Tamas Pusztai, Gyorgy Tegze, James A. Warren, and Jack F. Douglas. 2005. "Growth and Form of Spherulites." Physical Review E Statistical, Nonlinear, and Soft Matter Physics 72 (1): 1-15. doi:10.1103/PhysRevE.72.011605.

Granasy, Laszlo, Laszlo Ratkai, Attila Szallas, Balint Korbuly, Gyula I. Toth, Laszlo Kornyei, and Tamas Pusztai. 2014. "Phase-Field Modeling of Polycrystalline Solidification: From Needle Crystals to Spherulites - A Review." Metallurgical and Materials Transactions A: Physical Metallurgy and Materials Science 45 (4): 1694-1719. doi:10.1007/s11661-013-1988-0.

Green, Martin A., Yoshihiro Hishikawa, Ewan D. Dunlop, Dean H. Levi, Jochen Hohl-Ebinger, and Anita W.Y. Ho-Baillie. 2018. "Solar Cell Efficiency Tables (Version 51)." Progress in Photovoltaics: Research and Applications 26 (1): 312. doi:10.1002/pip.2978.

Higgins, Anthony M., Simon J. Martin, Mark Geoghegan, Sasha Y. Heriot, Richard L. Thompson, Robert Cubitt, Robert M. Dalgliesh, Ilaria Grizzi, and Richard A L Jones. 2006. "Interfacial Structure in Conjugated Polymers: Characterization and Control of the Interface between Poly(9,9-Dioctylfluorene) and Poly(9,9Dioctylfluorene- Alt-Benzothiadiazole)." Macromolecules 39 (19): 6699-6707. doi:10.1021/ma060072w.

Higgins, Julia S., and Henri C. Benoit. 1994. Polymers and Neutron Scattering. Oxford University Press.

Hopkinson, Pe, Pa Staniec, Andrew J. Pearson, Alan D F Dunbar, Tao Wang, Anthony J. Ryan, Richard A.L Jones, David G. Lidzey, and Athene M. Donald. 2011. "A Phase Diagram of the P3HT: PCBM Organic Photovoltaic System: Implications for Device Processing and Performance." Macromolecules 44: 2908-17. doi:10.1021/ma102524a.

James, David, Anthony M. Higgins, Paul Rees, Mark Geoghegan, M. Rowan Brown, Shion-Seng Chang, Dyfrig Môn, Robert Cubitt, Robert Dalgliesh, and Philipp Gutfreund. 2015. "Measurement of Molecular Mixing at a Conjugated Polymer Interface by Specular and Off-Specular Neutron Scattering." Soft Matter 11 (48). Royal Society of Chemistry: 9393-9403. doi:10.1039/C5SM02008E.

James, David William. 2011. "Characterisation of Buried Conjugated Polymer Interfaces by Off Specular Neutron Scattering, PhD Thesis, Swansea University."

Joanny, J F. 1978. "Critical Properties of a System of Two Molten Polymers." Journal of Physics A:Math. Gen. 11 (5): 4-8.

Jones, RAL. 2002. Soft Condensed Matter. Oxford University Press.

Jones, Richard A.L, and W. Richards, Randall. 1999. Polymers at Surfaces and Interfaces. Cambridge University Press.

Keddie, Joseph L., RAL Jones, and Rachel A. Cory. 1994. "Interface and Surface Effects on the Glass-Transition Temperature in Thin Polymer Films." Faraday Discussions.

Kienzle, Paul. 2016. "NIST Neutron SLD Calculator.” Mational Institue of Standards and Technology. https://www.ncnr.nist.gov/resources/activation/.

Kuang, Hao, Michael J Janik, and Enrique D Gomez. 2015. "Quantifying the Role of Interfacial Width on Intermolecular Charge Recombination in Block Copolymer Photovoltaics," 1-7. doi:10.1002/polb.23757. 
Kumar, Pankaj, Chhinder Bilen, Ben Vaughan, Xiaojing Zhou, Paul C. Dastoor, and Warwick J. Belcher. 2016. "Comparing the Degradation of Organic Photovoltaic Devices under ISOS Testing Protocols." Solar Energy Materials and Solar Cells 149. Elsevier: 179-86. doi:10.1016/j.solmat.2015.12.032.

Langer, J.S. 1980. "Instabilities and Pattern Formation in Crystal Growth." Reviews of Modern Physics 52.

Lee, Harrison Ka Hin, Andrew M. Telford, Jason A. Röhr, Mark F. Wyatt, Beth Rice, Jiaying Wu, Alexandre de Castro Maciel, et al. 2018. "The Role of Fullerenes in the Environmental Stability of Polymer:Fullerene Solar Cells." Energy \& Environmental Science 11. Royal Society of Chemistry: 417-28. doi:10.1039/C7EE02983G.

Lee, Hyun Hwi, Kwangseok Ahn, Doris Yangsoo Kim, Chung Jong Yu, and Dong Ryeol Lee. 2013. "Nanostructures of Small-Molecule Organic Crystals on Capillary Wave Surfaces with Controllable Capillary Lengths." Langmuir 29 (8): 2646-50. doi:10.1021/la304346a.

Lee, Kwan H., Paul E. Schwenn, Arthur R G Smith, Hamish Cavaye, Paul E. Shaw, Michael James, Karsten B. Krueger, Ian R. Gentle, Paul Meredith, and Paul L. Burn. 2011. "Morphology of All-Solution-Processed 'Bilayer' Organic Solar Cells." Advanced Materials 23: 766-70. doi:10.1002/adma.201003545.

Li, Gui, Guanghao Lu, Sijun Li, Haowei Tang, and Xiaoniu Yang. 2008. "EpitaxyAssisted Creation of PCBM Nanocrystals and Its Application in Constructing Optimized Morphology for Bulk-Heterojunction Polymer Solar Cells." Journal of Physical Chemistry B 112: 15651-58. doi:10.1021/jp8081529.

Li, Zhe, Him Cheng Wong, Zhenggang Huang, Hongliang Zhong, Ching Hong Tan, Wing Chung Tsoi, Ji Seon Kim, James R Durrant, and João T Cabral. 2013. "Performance Enhancement of Fullerene-Based Solar Cells by Light Processing." Nature Communications 4: 2227. doi:10.1038/ncomms3227.

Lindqvist, Camilla, Anke Sanz-Velasco, Ergang Wang, Olof Bäcke, Stefan Gustafsson, Eva Olsson, Mats R. Andersson, and Christian Müller. 2013. "Nucleation-Limited Fullerene Crystallisation in a Polymer-fullerene BulkHeterojunction Blend." Journal of Materials Chemistry A 1 (24): 7174. doi:10.1039/c3ta11018d.

Lorenzo, Arnaldo T., María Luisa Arnal, Julio Albuerne, and Alejandro J. Müller. 2007. "DSC Isothermal Polymer Crystallization Kinetics Measurements and the Use of the Avrami Equation to Fit the Data: Guidelines to Avoid Common Problems." Polymer Testing 26 (2): 222-31. doi:10.1016/j.polymertesting.2006.10.005.

Mailhot, Bénédicte, and Jean-luc Gardette. 1992. "Polystyrene Photooxidation. 1. Identification of the IR-Absorbing Photoproducts Formed at Short and Long Wavelenghts." Macromolecules 25 (16): 4119-26. doi:10.1021/ma00042a012.

Melosivic, Milan. 2012. Internal Reflection and ATR Spectroscopy.

Môn, Dyfrig, Anthony M. Higgins, David James, Mark Hampton, J. Emyr

Macdonald, Michael B. Ward, Philipp Gutfreund, Samuele Lilliu, and Jonathan Rawle. 2015. "Bimodal Crystallization at Polymer-fullerene Interfaces." Phys. Chem. Chem. Phys. 17. Royal Society of Chemistry: 2216-27. doi:10.1039/C4CP04253K.

Murphy, Douglas B., and Micheal W. Davidson. 2013. Fundamentals of Light Microscopy and Electronic Imaging. Wiley-Blackwell. 
Ngo, Trinh Tung, Duc Nghia Nguyen, and Van Tuyen Nguyen. 2012. "Glass

Transition of PCBM, P3HT and Their Blends in Quenched State." Advances in Natural Sciences: Nanoscience and Nanotechnology 3 (4): 045001. doi:10.1088/2043-6262/3/4/045001.

Perrin, Andrea, Osama M. Musa, and Jonathan W. Steed. 2013. "The Chemistry of Low Dosage Clathrate Hydrate Inhibitors." Chemical Society Reviews.

Polman, Albert, Mark Knight, Erik C Garnett, Bruno Ehrler, and Wim C Sinke. 2016. "Photovoltaic Materials - Present Efficiencies and Future Challenges" 352 (6283): 1-24. doi:10.1126/science.aad4424.

Pye, Justin E., and Connie B. Roth. 2015. "Above, below, and in-between the Two Glass Transitions of Ultrathin Free-Standing Polystyrene Films: Thermal Expansion Coefficient and Physical Aging." Journal of Polymer Science Part B: Polymer Physics 53 (1): 64-75. doi:10.1002/polb.23635.

R. Colby; M. Rubinstein; 2003. Polymer Physics. Oxford University Press.

R. Kelsall, I Hamley, M. Geoghan. 2005. "Scanning Probe Techniques." In Nanoscale Science and Technology, 82-87. John Wiley \& Sons.

Ragoussi, Maria-Eleni, and Tomás Torres. 2015. "New Generation Solar Cells: Concepts, Trends and Perspectives." Chem. Commun., 3957-72. doi:10.1039/C4CC09888A.

Rieger, J. 1996. "The Glass Transition Temperature of Polystyrene." Journal of Thermal Analysis 46.

Ro, Hyun Wook, Bulent Akgun, Brendan T. O’Connor, Matthew Hammond, R. Joseph Kline, Chad R Snyder, Sushil K. Satija, et al. 2012. "Poly(3Hexylthiophene) and [6,6]-Phenyl-C 61 -Butyric Acid Methyl Ester Mixing in Organic Solar Cells." Macromolecules 45 (16): 6587-99. doi:10.1021/ma3008527.

Roth, Connie B., and John R. Dutcher. 2005. "Glass Transition and Chain Mobility in Thin Polymer Films.” Journal of Electroanalytical Chemistry 584 (1): 13-22. doi:10.1016/j.jelechem.2004.03.003.

Russell, T. P. 1996. "On the Reflectivity of Polymers: Neutrons and X-Rays." Physica B: Condensed Matter 221 (1-4): 267-83. doi:10.1016/09214526(95)00937-X.

Saeki, Susumu. 1997. "Calculation of Combinatory Entropy in Complex Polymer Solutions Based on the Flory-Huggins Theory." Fluid Phase Equilibria 136 (12): 79-85. doi:10.1016/S0378-3812(97)00111-8.

Santangelo, P.G., and C.M. Roland. 1998. "Molecular Weight Dependence of Fragility in Polymers." Macromolecules 31: 4851-4585. doi:10.1021/ma900666z.

Seemann, Andrea, Tobias Sauermann, Christoph Lungenschmied, Oskar Armbruster, Siegfried Bauer, H. J. Egelhaaf, and Jens Hauch. 2011. "Reversible and Irreversible Degradation of Organic Solar Cell Performance by Oxygen." Solar Energy 85 (6). Elsevier Ltd: 1238-49. doi:10.1016/j.solener.2010.09.007.

Sferrazza, M., C. Xiao, R. A. L. Jones, D. Bucknall, J. Webster, and J. Penfold. 1997. "Evidence for Capillary Waves at Immiscible Polymer/Polymer Interfaces." Physical Review Letters 78 (19): 3693-96. doi:10.1103/PhysRevLett.78.3693.

Sharp, J. S., and J. a. Forrest. 2003. "Free Surfaces Cause Reductions in the Glass Transition Temperature of Thin Polystyrene Films." Physical Review Letters 91 (23): 235701. doi:10.1103/PhysRevLett.91.235701. 
Sinha, Moitreyee, and Donald J Buckley. 2007. Physical Properties of Polymers Handbook. Vol. 12309. doi:10.1007/978-0-387-69002-5.

Sivia, D.S. 2011. Elementary Scattering Theory for X-Ray and Neutron Users. Oxford University Press.

Spanggaard, Holger, and Frederik C. Krebs. 2004. "A Brief History of the Development of Organic and Polymeric Photovoltaics." Solar Energy Materials and Solar Cells 83: 125-46. doi:10.1016/j.solmat.2004.02.021.

Spectra-Tech. 1990. "Introduction to Attenuated Total Internal Reflectance (ATR)." FT-IR Technical Note TN-1, Brochure.

Speller, E. M. 2016. "The Significance of Fullerene Electron Acceptors in Organic Solar Cell Photo-Oxidation.” Materials Science and Technology 0 (0). Taylor \& Francis: 1-10. doi:10.1080/02670836.2016.1215840.

Speller, Emily M., James D. McGettrick, Beth Rice, Andrew M. Telford, Harrison K.H. Lee, Ching-Hong Tan, Catherine S. De Castro, et al. 2017. "The Impact of Nanomorphology on the Photochemistry of Fullerene Films: Correlating Stability to Triplet Exciton Kinetics." ACS Applied Materials \& Interfaces, acsami.7b03298. doi:10.1021/acsami.7b03298.

Spoltore, Donato, Tim Vangerven, Pieter Verstappen, Fortunato Piersimoni, Sabine Bertho, Koen Vandewal, Niko Van den Brande, et al. 2015. "Effect of Molecular Weight on Morphology and Photovoltaic Properties in P3HT:PCBM Solar Cells." Organic Electronics 21. Elsevier B.V.: 160-70. doi:10.1016/j.orgel.2015.02.017.

Sun, Yue, Yan Chun Han, and Jian Gang Liu. 2013. "Controlling PCBM Aggregation in P3HT/PCBM Film by a Selective Solvent Vapor Annealing." Chinese Science Bulletin 58 (22): 2767-74. doi:10.1007/s11434-013-5944-6.

Swinnen, Ann, Ilse Haeldermans, Martin Vande Ven, Jan D'Haen, Geert Vanhoyland, Stefano Aresu, Marc D'Olieslaeger, and Jean Manca. 2006. "Tuning the Dimensions of C60-Based Needlelike Crystals in Blended Thin Films." Advanced Functional Materials 16 (6): 760-65. doi:10.1002/adfm.200500812.

Tang, Hai, and Karl F. Freed. 1991. "Interfacial Studies of Incompressible Binary Blends." The Journal of Chemical Physics 94: 6307. doi:10.1063/1.460419.

Teran-Escobar, Gerardo, David M Tanenbaum, Eszter Voroshazi, Martin Hermenau, Kion Norrman, Matthew T Lloyd, Yulia Galagan, et al. 2012. "TOF-SIMS Investigation of Degredation Pathways Occurring in a Variety of Organic Photovoltaic Devices- the ISOS-3 Inter-Laboratory Collaboration." Physical Chemistry Chemical Physics : PCCP 14 (33): 11824-45. doi:10.1039/c2cp40821j.

Thakral, Seema, and RM Mehta. 2006. "Fullerenes: An Introduction and Overview of Their Biological Properties." Indian Journal of Pharmaceutical Sciences 68 (1): 13. doi:10.4103/0250-474X.22957.

Van Bavel, Svetlana, Erwan Sourty, Gijsbertus De With, Kai Frolic, and Joachim Loos. 2009. "Relation between Photoactive Layer Thickness, 3D Morphology, and Device Performance in P3HT/PCBM Bulk-Heterojunction Solar Cells." Macromolecules 42: 7396-7403. doi:10.1021/ma900817t.

Verploegen, Eric, Rajib Mondal, Christopher J. Bettinger, Seihout Sok, Michael F. Toney, and Zhenan Bao. 2010. "Effects of Thermal Annealing upon the Morphology of Polymer-Fullerene Blends." Advanced Functional Materials 20: 3519-29. doi:10.1002/adfm.201000975. 
Volonakis, G., L. Tsetseris, and S. Logothetidis. 2012. "Continuous Transformation Paths for the Molecular Crystals of the PCBM Fullerene Derivative." Synthetic Metals 162 (24). Elsevier B.V.: 2421-27. doi:10.1016/j.synthmet.2012.12.002.

Wang, Zhen Gang. 2002. "Concentration Fluctuation in Binary Polymer Blends: Chi Parameter, Spinodal and Ginzburg Criterion.” Journal of Chemical Physics 117 (1): 481-500. doi:10.1063/1.1481761.

Wong, Him Cheng, Zhe Li, Ching Hong Tan, Hongliang Zhong, Zhenggang Huang, Hugo Bronstein, Iain McCulloch, João T. Cabral, and James R. Durrant. 2014. "Morphological Stability and Performance of Polymer-Fullerene Solar Cells under Thermal Stress: The Impact of Photoinduced PC60BM Oligomerization." ACS Nano 8 (2): 1297-1308. doi:10.1021/nn404687s.

Woo, Claire H., Barry C. Thompson, Bumjoon J. Kim, Michael F. Tone, and Jean M. J. Fréchet. 2008. "The Influence of Poly(3-Hexylthiophene) Regioregularity on Fullerene-Composite Solar Cell Performance." Journal of the American Chemical Society.

Wypych, George. 2012. Polymer Handbook. ChemTech Publishing.

Yang, Xn, Jkj van Duren, Mt Rispens, Jc Hummelen, Raj Janssen, Maj Michels, and

J Loos. 2004. "Crystalline Organization of a Methanofullerene as Used for Plastic Solar-Cell Applications," no. 9: 802-6. doi:10.1002/adma.200306372.

Ye, Long, Huawei Hu, Masoud Ghasemi, Tonghui Wang, Brian A. Collins, Joo Hyun Kim, Kui Jiang, et al. 2018. "Quantitative Relations between Interaction Parameter, Miscibility and Function in Organic Solar Cells." Nature Materials 17 (3). Springer US: 253-60. doi:10.1038/s41563-017-0005-1.

Zhang, Xiaowei, Nian Ji1, Valeria Lauter, Haile Ambaye, and Jian-Ping Wang. 2016. Neutron Scattering. intechopen.

Zhang, Yiwei, Edward Bovill, James Kingsley, Alastair R. Buckley, Hunan Yi, Ahmed Iraqi, Tao Wang, and David G. Lidzey. 2016. "PCDTBT Based Solar Cells: One Year of Operation under Real-World Conditions." Scientific Reports 6. Nature Publishing Group: 4-11. doi:10.1038/srep21632.

Zhao, Jun, Ann Swinnen, Guy Van Assche, Jean Manca, Dirk Vanderzande, and Bruno Van Mele. 2009. "Phase Diagram of P3HT/PCBM Blends and Its Implication for the Stability of Morphology." Journal of Physical Chemistry B 113: 1587-91. doi:10.1021/jp804151a.

Zhao, Yinyan, Petra Inbar, Hitesh P. Chokshi, A. Waseem Malick, and Duk Soon Choi. 2011. "Predictions of the Thermal Phase Diagram of Amorphous Solid Dispersions by Flory-Huggins Theory." International Journal of Drug Development and Research 3 (2): 26-33. doi:10.1002/jps.

Zheng, Lidong, Jiangang Liu, Yan Ding, and Yanchun Han. 2011. "Morphology

Evolution and Structural Transformation of Solution-Processed Methanofullerene Thin Film under Thermal Annealing." Journal of Physical Chemistry B 115: 8071-77. doi:10.1021/jp2030279.

Zhuang, Taojun, Xiao-Feng Wang, Takeshi Sano, Ziruo Hong, Yang Yang, and Junji Kido. 2013. "Fullerene Derivatives as Electron Donor for Organic Photovoltaic Cells.” Applied Physics Letters 103 (20): 203301. doi:10.1063/1.4830037. 